Pacific Journal of Mathematics

NONSOLVABLE FINITE GROUPS ALL OF WHOSE LOCAL 


\title{
NONSOLVABLE FINITE GROUPS ALL OF WHOSE LOCAL SUBGROUPS ARE SOLVABLE, II
}

\author{
John G. THOMPson
}

\begin{abstract}
In this second paper, the bulk of the work is devoted to characterizing $E_{2}(3)$ and $S_{4}(3)$. These two groups are "almost" $N$-groups and it is relevant to treat them separately. The actual characterizations (Theorems 8.1 and 9.1) are very technical but the hypotheses deal with the structure and embedding in a simple group of certain $\{2,3\}$-subgroups.
\end{abstract}

This paper is a continuation of an earlier paper. ${ }^{1}$ The bibliographical references are to $\mathrm{I}$.

7. Groups in which 1 is the only $p$-signalizer.

Definition 7.1. $\mathscr{Q}^{*}(p)=\{\mathfrak{B} \mid$ (i) $\mathfrak{B}$ is a subgroup of $\mathbb{B}$ of type $(p, p)$. (ii) $N(\mathfrak{B})$ contains a $S_{p}$-subgroup of $(\mathcal{S}$.$\} .$

Hypothesis 7.1. ( i ) $p$ is a prime and if $\mathfrak{B} \in \mathscr{Q}^{*}(p)$, then no $S_{p}$-subgroup of $C(\mathfrak{B})$ normalizes any nonidentity $p^{\prime}$-subgroup of (B).

(ii) The centralizer of every nonidentity $p$-subgroup of $\mathbb{B}$ is $p$ solvable.

Lemmas 7.1, 7.2, 7.3 are proved under Hypothesis 7.1.

Lemma 7.1. ( i ) $\mathscr{U}(p) \subseteq \mathscr{E}(p)$. (See Definitions 2.8 and 2.10 of I). (ii) If $p \geqq 5$, then $\mathscr{U}^{*}(p) \subseteq \mathscr{E}(p)$.

(iii) If $p=3$ and if no element of $\mathscr{U}(3)$ centralizes a quaternion subgroup of $\left(\mathbb{S}\right.$, then $\mathscr{C}^{*}(3) \subseteq \mathscr{E}(3)$.

Proof. If $p$ is odd, choose $\mathfrak{B} \in \mathscr{C}^{*}(p)$, while if $p=2$, choose $\mathfrak{B} \in \mathscr{C}(2)$. We must show that either $\mathfrak{B}$ centralizes every element of И(B; $\left.p^{\prime}\right)$ or $p=3, \mathfrak{B} \in \mathscr{U}^{*}(3)-\mathscr{U}(3)$ and some element of $\mathscr{U}(3)$ centralizes a quaternion subgroup of (S).

Let $\mathfrak{P}$ be a $S_{p}$-subgroup of $N(\mathfrak{B})$, so that $\mathfrak{P}$ is a $S_{p}$-subgroup of (3. Proceeding by way of contradiction, let $\mathfrak{\Omega}$ be an element of $И\left(\mathfrak{B} ; p^{\prime}\right)$ minimal subject to $[\mathfrak{Q}, \mathfrak{B}] \neq 1$. Then $\mathfrak{Q}$ is a $q$-group for some prime

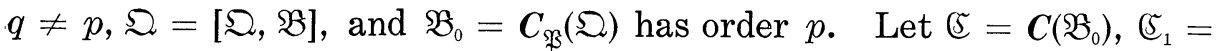
$C_{\mathfrak{P}}\left(\mathfrak{B}_{0}\right)$, and let $\mathfrak{P}^{*}$ be a $S_{p}$-subgroup of $\sqrt{5}$ containing $\mathfrak{S}_{1}$. Hypothesis 7.1 implies that $\mathfrak{O}_{p^{\prime}}(\mathfrak{S})=1$. Let $\mathfrak{P}_{0}=\boldsymbol{O}_{p}(\mathfrak{S})$. If $\left[\mathfrak{P}_{0}, \mathfrak{B}\right] \subseteq \mathfrak{B}$, then

1 Non-solvable finite groups all of whose local subgroups are solvable, I, Bull. Amer. Math. Soc. 74 (1968), 383-437, which will be referred to as I. 
Lemma 5.16 is violated. Hence, we have $\left|\mathfrak{P}^{*}: \mathfrak{F}_{1}\right|=\left|\mathfrak{P}_{0}: \mathfrak{P}_{0} \cap \mathfrak{S}_{1}\right|=p$ and $\left[\mathfrak{B}_{0}, \mathfrak{B}\right] \nsubseteq \mathfrak{B}$.

Suppose $\mathfrak{B} \subseteq \mathfrak{P}_{0}$. Then $\mathfrak{Q}=[\mathfrak{Q}, \mathfrak{B}] \subseteq \mathfrak{P}_{0}$, so $\mathfrak{Q}=1$. This is not the case, so $\mathfrak{B} \nsubseteq \mathfrak{P}_{0}$. By Lemma 6.1, it follows that $\mathfrak{B} \notin \mathscr{C}(p)$. Hence,

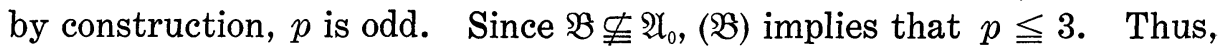
$p=3$ and $\mathfrak{B} \in \mathscr{U}^{*}(3)-\mathscr{U}(3)$. By definition of $\mathscr{U}(3)$ and $\mathscr{U}^{*}(3)$, it follows that $Z(\mathfrak{P})$ is non cyclic and $\mathfrak{B}$ is not contained in the center of any $S_{3}$-subgroup of $\&$.

Since $\left[\mathfrak{P}_{0}, \mathfrak{B}, \mathfrak{B}\right]=1$ and since $\mathfrak{B} \varsubsetneqq \mathfrak{P}_{0}$, it follows that $\mathfrak{B} \nsubseteq \boldsymbol{O}_{3}\left(\mathfrak{C}_{2,3}\right)$, where $\mathfrak{F}_{2,3}$ is a $S_{2,3}$-subgroup of $\sqrt{5}$ containing $\mathfrak{S}^{*}$.

Since $\mathfrak{B}_{0} \nsubseteq Z(\mathfrak{P})$, we have $\mathfrak{B}=\mathfrak{B}_{0} \times \mathfrak{B}_{1}$, where $\mathfrak{B}_{1} \subseteq Z(\mathfrak{P})$. Since $\mathfrak{B}_{0} \subseteq Z(\mathfrak{E})$, we have $\mathfrak{B}_{0} \subseteq O_{3}\left(\mathfrak{F}_{2,3}\right)$, and so $\mathfrak{B}_{1} \nsubseteq O_{3}\left(\mathfrak{F}_{2,3}\right)$.

Let $\mathfrak{F}$ be a subgroup of $\mathfrak{F}_{2,3}$ such that

(a) $\mathfrak{P}^{*} \subseteq \mathfrak{K}$.

(b) $\mathfrak{B}_{1} \nsubseteq \boldsymbol{O}_{3}(\mathfrak{S})$.

(c) $\mathfrak{S}$ is minimal subject to (a) and (b).

Let $\mathfrak{S}_{1}=\boldsymbol{O}_{3}(\mathfrak{F})$. Since the fixed point subspace of $\mathfrak{B}_{1}$ on $\mathfrak{S}_{1} / \boldsymbol{D}\left(\mathfrak{S}_{1}\right)$ is of codimension 1, Lemma 5.30 implies that $\mathfrak{S}=\mathfrak{P}^{*} \mathfrak{\Omega}^{*}$, where $\mathfrak{\Omega}^{*}$ is a quaternion group and $\left|\mathfrak{F}^{*}: \mathfrak{S}_{1}\right|=3$, so that $\mathfrak{S}_{1} \mathfrak{B}_{1}=\mathfrak{P}^{*}$. Since $\mathfrak{B}_{1}$ centralizes $\boldsymbol{D}\left(\mathfrak{S}_{1}\right)$, so does $\mathfrak{Q}^{*}$. Let $\boldsymbol{C}_{\mathfrak{S}_{1}}\left(\mathfrak{\Omega}^{*}\right)=\mathfrak{P}_{1}^{*}$. Thus, $\mathfrak{P}_{1}$ is a normal subgroup of $\mathfrak{F ^ { \prime }}$ and $\left|\mathscr{F}_{1}: \mathfrak{P}_{1}^{*}\right|=9$.

Let $\mathfrak{P}_{2}^{*}=\left[\mathfrak{S}_{1}, \mathfrak{\Omega}^{*}\right]$. Then $\mathfrak{P}_{2}^{*}$ is generated by 2 elements and $\mathfrak{P}_{2}^{*} \cap \mathfrak{P}_{1}^{*}$ is of index 9 in $\mathfrak{P}_{2}^{*}$. Hence, $\mathfrak{P}_{2}^{*}$ is either elementary of order 9 or is a nonabelian group of exponent 3 and order 27. Furthermore, $\mathfrak{S}_{1}=\mathfrak{P}_{1}^{*} \mathfrak{P}_{2}^{*}, \mathfrak{P}_{1}^{*} \cap \mathfrak{P}_{2}^{*}=\boldsymbol{D}\left(\mathfrak{P}_{2}^{*}\right)$, and $\left[\mathfrak{P}_{1}^{*}, \mathfrak{P}_{2}^{*}\right]=1$.

Since $\mathfrak{B}_{1} \nsubseteq \mathfrak{S}_{\mathfrak{Z}_{1}}$, it follows that $\mathfrak{S}_{1}=\mathfrak{B}_{1} \times\left(\mathfrak{S}_{1} \cap \mathfrak{S}_{1}\right)$. Hence, $\boldsymbol{D}\left(\mathfrak{C}_{1}\right)=$ $\boldsymbol{D}\left(\mathfrak{S}_{1} \cap \mathfrak{S}_{1}\right) \subseteq \mathfrak{P}_{1}^{*}$. We will show that $\boldsymbol{D}\left(\mathfrak{S}_{1}\right)=1$. Suppose false. Let $\mathfrak{C}^{*}=\boldsymbol{C}\left(\boldsymbol{D}\left(\mathfrak{\Im}_{1}\right)\right)$, so that $\mathfrak{C}^{*}$ is 3 -solvable. Since $\mathfrak{C}^{*} \triangleleft \boldsymbol{N}\left(\boldsymbol{D}\left(\mathfrak{C}_{1}\right)\right)$, it follows that $\mathfrak{C} * \mathfrak{P}$ is 3 -solvable. Since $\mathfrak{B}_{1} \subseteq Z(\mathfrak{P})$, we have $\mathfrak{B}_{1} \subseteq O_{3}\left(\mathfrak{C}^{*} \mathfrak{R}\right)$. Since $\mathfrak{\Omega}^{*}$ centralizes $\boldsymbol{D}\left(\mathfrak{S}_{1}\right)$, it follows that $\left\langle\mathfrak{B}_{1}, \mathfrak{Q}^{*}\right\rangle \subseteq \mathfrak{C}^{*}$. Thus, $\left\langle\mathfrak{B}_{1}, \mathfrak{Q}^{*}\right\rangle$ is 3 -closed. This is impossible, since $\left\langle\mathfrak{B}_{1}, \mathfrak{Q}^{*}\right\rangle$ covers $\mathfrak{F}_{\mathfrak{C}} / \mathfrak{F}_{\mathfrak{1}}$.

If $\mathfrak{P}_{2}^{*}$ is nonabelian, then $\mathfrak{\Omega}^{*}$ centralizes $Z\left(\mathfrak{P}^{*}\right)$. Since $\mathfrak{\Omega}^{*}$ is a quaternion group, we are done in this case.

We may now assume that $\mathfrak{P}_{2}^{*}$ is abelian, so elementary of order 9 . Thus, $\mathfrak{S}_{1}=\mathfrak{P}_{1}^{*} \times \mathfrak{P}_{2}^{*}, \mathfrak{P}_{1}^{*}$ and $\mathfrak{S}_{1}$ are elementary and $\boldsymbol{Z}\left(\mathfrak{B}^{*}\right)=\mathfrak{P}_{1}^{*} \times \mathfrak{B}$, where $\mathfrak{B}=\mathfrak{P}_{2}^{*} \cap \boldsymbol{Z}\left(\mathfrak{P}^{*}\right)$. Notice that $\mathfrak{B}_{0} \subseteq \mathfrak{P}_{1}^{*}$. If $\mathfrak{P}_{1}^{*} \supset \mathfrak{B}_{0}$, then since every subgroup of $\mathfrak{P}_{1}^{*}$ of type $(3,3)$ is in $\mathscr{C}_{\mathcal{L}}(3)$ and since the quaternion group $\Omega^{*}$ centralizes $\mathfrak{P}_{1}^{*}$, we are done. We may therefore assume that $\mathfrak{P}_{1}^{*}=\mathfrak{B}_{0}$. Hence, $\boldsymbol{Z}(\mathfrak{P})$ has order $9,\left|\mathfrak{P}^{*}\right|=3^{4},\left|\mathfrak{S}_{1}\right|=3^{3}$. Also, $\boldsymbol{Z}\left(\mathfrak{P}^{*}\right)=\mathfrak{B}_{0} \times \mathfrak{B}$. Let $B$ be a generator for $\mathfrak{B}_{0}$ and let $I$ be the involution of $\mathfrak{\Omega}^{*}$. Then $I$ inverts $\mathfrak{B}$ and centralizes $B$.

Let $\mathfrak{N}=\left\langle\mathfrak{P}, \mathfrak{P}^{*}\right\rangle \leqq N\left(\mathfrak{S}_{1}\right)$. Since $S L(3,3)$ is a minimal simple group, it follows that $N\left(\mathfrak{S}_{1}\right)$ is solvable. As $\boldsymbol{O}_{3^{\prime}}(\mathfrak{R})=1$, we have $\mathfrak{S}_{1}=\boldsymbol{C}\left(\mathfrak{S}_{1}\right)$. Since $\left[\mathfrak{E}_{1}, \mathfrak{P}^{*}, \mathfrak{P}^{*}\right]=1$, it follows that $\mathfrak{R}$ contains a normal subgroup 
$\mathfrak{B}^{*}$ of order 3 and that $S_{2}$-subgroups of $\mathfrak{N}$ are quaternion. Now $\mathfrak{B}^{*} \nsubseteq \mathfrak{B}$, since $\mathfrak{P}$ does not normalize $\mathfrak{B}_{0}$ and $\mathfrak{P}^{*}$ centralizes no subgroup of $\mathfrak{B}$ other than 1 and $\mathfrak{B}_{0}$. Suppose $\mathfrak{B}^{*}=\mathfrak{B}$. Since $\mathfrak{B}=\mathfrak{P}^{* \prime}$, it follows that $S_{3}$-subgroups of $\mathfrak{R} / \mathfrak{B}^{*}$ are abelian. Thus $\mathfrak{N}$ is 3 -closed. But this is impossible since $\mathfrak{P} \neq \mathfrak{P}^{*}$. Hence, $\mathfrak{B}^{*}$ is a subgroup of $Z\left(\mathfrak{P}^{*}\right)$ of order 3 which is different from $\mathfrak{B}$ and from $\mathfrak{B}_{0}$. Since $Z\left(\mathfrak{P}^{*}\right)=\mathfrak{B}_{0} \times \mathfrak{B}$, there is a generator for $\mathfrak{B}^{*}$ of the shape $B V$, where $V$ is a generator for $\mathfrak{B}$.

Let $J$ be any involution of $\mathfrak{R}$. Then $J V J=V^{-1}$ and $J B V J=B V$. Now $I$ and $J$ both normalize $\mathfrak{P}^{*}$ and $\mathfrak{P}^{* \prime}=\mathfrak{B}$. Hence, $\langle I, J\rangle$ maps onto an abelian subgroup of $\boldsymbol{A}_{\mathscr{S}}\left(\boldsymbol{Z}\left(\mathfrak{P}^{*}\right)\right)$, which implies that $J$ normalizes $Z\left(\mathfrak{P}^{*}\right) \cap C(I)=\mathfrak{B}_{0}$. Hence, $J B J=B^{f}$ for some integer $f$, and the previous equations yield $V^{2}=1$, which is not the case. The proof is complete.

Hypothesis 7.2. (i) If $\mathfrak{A} \in \mathscr{S}_{\text {cn }_{3}}(p)$, then $И(\mathfrak{U})$ contains only 1.

(ii) If 3 is of order $p$ and is in the center of some $S_{p}$-subgroup of $\mathbb{S}$, then $\boldsymbol{O}_{p}(\mathfrak{M})$ is of symplectic type and width $w$, where $\mathfrak{M}=N(3)$.

LEMMA 7.2. Suppose Hypothesis 7.2 is satisfied and that if $p=$ 2 , then $w \geqq 3$, while if $p=3$, then $w \geqq 2$. Let $\mathfrak{B}$ be a subgroup of $\boldsymbol{O}_{p}(\mathfrak{M})$ of type $(p, p)$ which contains 3. Then $\mathfrak{B} \in \mathscr{E}(p)$.

Proof. Let $\mathscr{Y}$, be the set of subgroups of $\boldsymbol{O}_{p}(\mathfrak{M})$ which violate the lemma. Let $\mathscr{V}_{0}$ be the subset of those $\mathfrak{B}$ in $\mathscr{V}^{-}$which centralize at least one element $\mathfrak{B}$ of $\mathscr{U}(p)$ which $3 \subset \mathfrak{B} \subset \boldsymbol{O}_{p}(\mathfrak{M})$. If $\mathscr{Y}{ }_{0} \neq \varnothing$, choose $\mathfrak{B} \in \mathscr{V}^{-}$, while if $\mathscr{V}^{0}{ }_{0}=\varnothing$, choose $\mathfrak{B}$ in $\mathscr{V}^{\circ}$.

Let $\mathfrak{S}_{\mathfrak{Z}}=\boldsymbol{O}_{p}(\mathfrak{M}), \mathfrak{S}_{0}=\boldsymbol{C}_{\mathfrak{S}}(\mathfrak{B})$. We first argue that $\boldsymbol{U}\left(\mathfrak{S}_{0} ; p^{\prime}\right)$ is trivial. Namely, $\mathfrak{M}$ is $p$-solvable with $\boldsymbol{O}_{p}(\mathfrak{M})=1$, so $\boldsymbol{C}_{\mathfrak{M}}(\mathfrak{S})=3(\mathfrak{S})$. This implies that $C_{\mathfrak{M}}\left(\mathfrak{S}_{0}\right)$ is a $p$-group. Hence, $\boldsymbol{И}_{\mathfrak{M}}\left(\mathfrak{L}_{0} ; p^{\prime}\right)$ is trivial. Suppose $\mathfrak{R} \in \boldsymbol{U}\left(\mathfrak{S}_{0} ; p^{\prime}\right)$. It suffices to show that $\mathfrak{R} \subseteq \mathfrak{M}$. If $\mathfrak{S}_{0}$ contains an element $\mathfrak{B}$ of $\mathscr{\mathscr { C }}(p)$ with $3 \subset \mathfrak{B}$, then by Lemma 7.1 we get that $\mathfrak{B}$ centralizes $\mathfrak{R}$. Hence, $\mathfrak{R} \subseteq C(\mathbb{B}) \cong \mathfrak{M}$. If no such elements of $\mathscr{C}(p)$ are available, then by construction, $\mathscr{V}_{0}=\varnothing$. But $\mathfrak{S} \triangleleft \mathfrak{M}$, so if $\mathfrak{P}$ is a $S_{p}$-subgroup of $\mathfrak{M}$, then $\mathfrak{S}$ contains an element $\mathfrak{B}$ of $\mathscr{U}(\mathfrak{P})$. Let $\mathfrak{S}_{1}=C_{\mathfrak{F}}(\mathfrak{B})$ so that $\left|\mathfrak{S}_{\mathfrak{2}}: \mathfrak{F}_{1}\right|=p$. If $\mathfrak{S}_{0} \cap \mathfrak{S}_{1}$ contains more than one subgroup of order $p$, then there is a subgroup $\mathfrak{B}^{*}$ of $\mathfrak{S}_{0} \cap \mathfrak{S}_{1}$ of type $(p, p)$ which contains 3. Since $\mathscr{Y}_{0}=\varnothing, \mathscr{V}^{*} \in \mathscr{E}(p)$, so $\mathfrak{R} \subseteq C\left(\mathfrak{B}^{*}\right) \subseteq$ $\boldsymbol{C}(3) \subseteq \mathfrak{M}$. Suppose $\mathfrak{S}_{0} \cap \mathfrak{S}_{1}$ contains only one subgroup of order $p$. Then by hypothesis, we have $p \geqq 5$, and so $\mathfrak{S}$ is of width 1 and is a $S_{p}$-subgroup of $\mathbb{S}$. Hypothesis 7.1 guarantees in this case that $И\left(\mathfrak{F}_{0} ; p^{\prime}\right)$ is trivial, so $\Re=1$. We have thus shown that $И\left(\mathscr{\mathcal { F }}_{0} ; p^{\prime}\right)$ is trivial.

Choose $\mathfrak{Q}$ in $И\left(\mathfrak{B} ; p^{\prime}\right)$ minimal subject to $[\mathfrak{B}, \mathfrak{Q}] \neq 1$. Then $\mathfrak{Q}=$ $[\mathfrak{B}, \mathfrak{Q}]$ and $\mathfrak{B}_{0}=C_{\mathfrak{B}}(\mathfrak{Q})$ is of order $p$. Clearly, $\mathfrak{B}_{0} \neq \mathfrak{3}$. Let $\mathfrak{M}_{1}=N\left(\mathfrak{B}_{0}\right)$ 
so that $\mathfrak{M}_{1}$ is $p$-solvable. By the preceding argument, $\boldsymbol{O}_{p},\left(\mathfrak{M}_{1}\right)=1$. Hence, $3 \nsubseteq \boldsymbol{O}_{p}\left(\mathfrak{M N}_{1}\right)$, so that $\mathfrak{S}_{0}$ contains an extra special subgroup $\mathfrak{S}^{*}$ of width $w-1$ with $\mathfrak{F}^{*} \cap \boldsymbol{O}_{p}\left(\mathfrak{M}_{1}\right)=1$.

Let $\mathfrak{X}=\boldsymbol{R}_{p}\left(\mathfrak{M}_{1}\right)$ (see Definition 2.2), $\mathfrak{Y}=\boldsymbol{C}_{\mathfrak{M}_{1}}(\mathfrak{X})$. Suppose $3 \subseteq \mathfrak{Y}$. Since $\mathfrak{Z}=[\mathfrak{O}, \mathfrak{3}]$ and $\mathfrak{Y} \triangleleft \mathfrak{M}_{1}$, we have $\mathfrak{Q} \cong \mathfrak{Y}$. Let $\mathfrak{X}_{p}$ be a $S_{p^{-}}$ subgroup of $\mathfrak{M}_{1}$ which contains $\mathfrak{S}^{*}$. Then $\mathfrak{S}^{*}$ centralizes $\boldsymbol{Z}\left(\mathfrak{X}_{p}\right)$, so $\mathfrak{Y S} \mathfrak{S}^{*}$ centralizes $\boldsymbol{Z}\left(\mathfrak{X}_{p}\right)$. Let $\tilde{\mathfrak{P}}$ be a $S_{p}$-subgroup of $\mathbb{S}$ containing $\mathfrak{X}_{p}$. Then $\boldsymbol{Z}(\tilde{\mathfrak{B}}) \leqq Z\left(\mathfrak{X}_{p}\right)$, so $\mathfrak{S}^{*}$ is contained in a conjugate $\widetilde{\mathfrak{M}}$ of $\mathfrak{M}$, $\widetilde{\mathfrak{M}}=$ $N\left(\Omega_{1}(Z(\tilde{\mathfrak{B}}))\right)$. Furthermore, since $3 \mathfrak{Z} \subseteq \mathfrak{Y} \subseteq \widetilde{\mathfrak{M}}$, $\mathfrak{S}^{*}$ is faithfully repre-

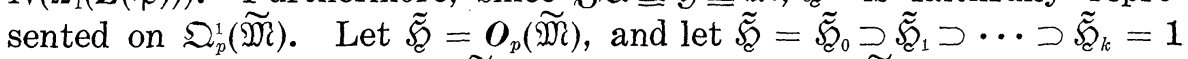
be part of a chief series for $\widetilde{\mathfrak{M}}$. Then since $3 \nsubseteq \boldsymbol{O}_{p}(\widetilde{\mathfrak{D}})$, it follows that 3 does not centralize $\tilde{\mathfrak{F}}_{n} / \tilde{\mathscr{S}}_{n+1}$ for at least one value of $n, 0 \leqq n<k$. Hence, $\left|\tilde{\mathfrak{F}}_{n}: \tilde{\mathfrak{F}}_{n+1}\right|=p^{a_{n}}$ where $a_{n} \geqq r_{p}(3 ; \widetilde{\mathfrak{M}})$. Then by Lemma 5.4, $r_{p}(3 ; \widetilde{\mathfrak{M}}) \geqq r_{c}(3 ; \tilde{\mathfrak{P}})$. Clearly,

$$
r_{c}(3 ; \tilde{\mathfrak{R}}) \geqq r_{c}\left(B ; \mathfrak{S}^{*}\right)=p^{w-1} .
$$

On the other hand, $\tilde{\mathfrak{F}}_{n}$ is a subgroup of $\tilde{\mathfrak{K}}$, so $2 w \geqq a_{n}$. Hence $2 w \geqq$ $p^{w-1}$. If $p \geqq 5$, then $w=1$ is forced, so every $p$-solvable subgroup of (8) has $p$-length at most 1 . This is absurd, so $p \leqq 3$. If $p=3$, then $w=2$, since $w \geqq 2$ by hypothesis. It is clear that this is impossible since $\mathfrak{S}^{*}$ is faithfully represented on $\boldsymbol{Q}_{3}^{1}(\widetilde{\mathfrak{W}})$. If $p=2$, then $w=3$ or $w=4$, since by hypothesis $w \geqq 3$. This is also impossible by Lemma 5.13. We have shown that $3 \nsubseteq \mathfrak{Y}$.

Since $\mathfrak{X}$ is a p-group, $\mathfrak{X} \subseteq \mathfrak{M}^{G}$ for some $G$ in $\mathfrak{B}$. Then $\mathfrak{X} \cap \mathfrak{S}^{G}$ is an abelian subgroup of $\mathfrak{S}^{G}$, so $m\left(\mathfrak{X} \cap \mathfrak{S C}^{G}\right) \leqq w+1+e$, where $e=0$ if $p$ is odd and $e=1$ if $p=2$.

If $p$ is odd, then $\mathfrak{X} / \mathfrak{X} \cap \mathscr{S}^{G}$ is faithfully represented on the Frattini quotient group of $\Omega_{1}\left(\mathfrak{S}^{G}\right)$, and this latter group is generated by $2 w$ elements. If $p=2$, write $\boldsymbol{O}_{2,2^{\prime}}\left(\mathfrak{M}^{a}\right)=\mathfrak{S C}^{a} \cdot \Re$ where $|\Re|$ is odd. Then $\left[\Re, \mathbb{S}^{a}\right]$ is generated by $2 w$ elements and $\mathfrak{X} / \mathfrak{X} \cap \mathfrak{S}^{G}$ is faithfully represented on the Frattini quotient group of $\left[\Re, \mathfrak{S}^{c}\right]$. Thus, by a result of Schur [32], we have $m\left(\mathfrak{X} / \mathfrak{X} \cap \mathfrak{M}^{G}\right) \leqq w^{2}$, that is,

$$
m(\mathfrak{X}) \leqq w^{2}+w+1+e .
$$

If $w=1$, then $p \geqq 5$ implies that $O_{p}(\mathfrak{M})$ is a $S_{p}$-subgroup of (S). So every $p$-solvable subgroup of $(5)$ has $p$-length at most 1 , a contradiction. Hence, $w \geqq 2$.

There is an elementary subgroup $\mathfrak{X}_{1}$ of $\mathfrak{X}$ such that $\boldsymbol{A}_{\mathscr{S}}\left(\mathfrak{X}_{1}\right)$ contains a subgroup $\tilde{\mathfrak{F}} * \widetilde{\mathfrak{n}}$, where $\widetilde{\mathfrak{D}} \triangleleft \widetilde{\mathfrak{F}} * \widetilde{\mathfrak{Q}}$ is special and $\tilde{\mathfrak{F}}^{*} \cong \mathfrak{F}^{*}$ operates faithfully and irreducibly on $\widetilde{\mathfrak{Q}} / \boldsymbol{D}(\tilde{\mathfrak{\Omega}})$. Also, $\tilde{\mathfrak{S}} * \tilde{\mathfrak{\Omega}}$ acts irreducibly on $\mathfrak{X}_{1}$.

Assume that $p$ is odd.

Since $\tilde{\mathfrak{S}}^{*}$ is extra special of width $w-1$, it follows that $m(\tilde{\mathfrak{\Omega}}) \geqq$ $p^{w-1} a$, where $a=\left|F_{q}(\zeta): F_{q}\right|$. Here, $\tilde{\mathfrak{Q}}$ is a $q$-group and $\zeta$ is a primitive 
$p^{\text {th }}$ root of 1 in an extension field of the prime field $F_{q}$. By Lemma 5.3, $m(\mathfrak{X}) \geqq m(\widetilde{\mathfrak{O}}) b$, where $b=2 / 3$ if $q=2$ and $b=\left|F_{p}(\tau): F_{p}\right|$ if $q$ is odd. Here $\tau$ is a primitive $q^{\text {th }}$ root of 1 in an extension field of the prime field $F_{p}$. Together with (7.1), we get $a b p^{w-1} \leqq w^{2}+w+1$. Clearly, $a b>1$. Suppose $w \geqq 4$. Then $3^{w-1} \leqq p^{w-1}<w^{2}+w+1$, a contradiction. Suppose $w=3$. If $p \geqq 5$, then $5^{2}=5^{w-1} \leqq p^{w-1}<3^{2}+$ $3+1$, a contradiction. Thus, $p=3$ and $q=2$. Since $p=3, w=3$, it follows that $\mathfrak{M}^{G} / \mathfrak{S}^{a}$ is isomorphic to a 3-solvable subgroup of the 6 by 6 symplectic group over $F_{3}$. It follows readily that $\mathfrak{M}^{a} / \mathfrak{S}^{a}$ has no elementary subgroup of order $3^{4}$. Thus, in this case, $m(\mathfrak{X}) \leqq 4+$ $+m\left(\mathfrak{X} \cap \mathfrak{S}_{\mathfrak{C}}{ }^{G}\right) \leqq 8$, against $(4 / 3) \cdot 3^{2}=12=a b p^{w-1} \leqq m(\mathfrak{X})$. Hence $w=$ 2. We now get $a b p^{w-1}<7$, so $p \leqq 5$. Suppose $p=5$ and $q$ is odd. Then $a b \geqq 2$, so that $10<7$. Suppose $p=5$ and $q=2$. Then $a=4$, so $a b=8 / 3$. We get $(8 / 3) \cdot 5<7$. Hence, $p=3$. Suppose $q$ is odd. Since $a$ is characterized as the smallest positive integer $n$ with $3^{n} \equiv$ $1(\bmod q)$, it follows that $a \geqq 3$, so $a b \geqq 3$. This gives $9 \leqq a b p^{w-1}<7$. Hence, $q=2$. Since $p=3, w=2$, it follows that $\mathfrak{M}^{G} / \mathfrak{S}^{G}$ has no elementary subgroup of order 27 . Hence, $m(\mathfrak{X}) \leqq 3+2=5$. In particular, $m\left(\mathfrak{X}_{1}\right) \leqq 5$. Suppose first that $\tilde{\mathfrak{D}}$ is abelian. Since $\boldsymbol{Z}\left(\tilde{\mathfrak{F}}^{*}\right)=\widetilde{\mathfrak{Z}}$ acts without fixed points on $\widetilde{\mathfrak{Q}}$, it follows that $\boldsymbol{C}_{\widetilde{\mathfrak{Z}}^{*}}(\lambda) \cap \widetilde{\mathfrak{Z}}=1$ for every non trivial character $\lambda$ of $\tilde{\mathfrak{\Omega}}$. So $\left|\tilde{\mathfrak{F}}^{*}: C_{\mathfrak{S}^{*}}(\lambda)\right| \geqq 9$ for all $\lambda \neq 1$. Hence, $m\left(\mathfrak{X}_{1}\right) \geqq 9$, a contradiction. Suppose $\widetilde{\mathfrak{D}}$ is nonabelian. Let $\mathfrak{X}_{2}$ be a subgroup of $\mathfrak{X}_{1}$ on which $\tilde{\mathfrak{D}}$ acts irreducibly. Thus $m\left(\mathfrak{X}_{2}\right) \geqq 2$, since $\tilde{\mathfrak{a}}^{\prime}$ does not centralize $\mathfrak{X}_{2}$. Since $m\left(\mathfrak{X}_{1}\right) \leqq 5$, and $p=3$, it follows that $\mathfrak{X}_{2}=\mathfrak{X}_{1}$ is an irreducible $\widetilde{\mathfrak{\Omega}}$-group. Thus, $\widetilde{\mathfrak{D}}$ is extra special. But $m(\tilde{\mathfrak{\Omega}})=6$, since $q=2$ and $\left|\tilde{\mathfrak{F}}^{*}\right|=27$. This yields $m\left(\mathfrak{X}_{1}\right) \geqq 2^{3}$. All possibilities have led to contradictions. So $p=2$.

Since $\widetilde{\mathfrak{S}}^{*}$ is extra special of width $w-1$, we get that $m(\widetilde{\beth}) \geqq 2^{w-1}$. Now Lemma $5.3\left(\right.$ a) applied with $\widetilde{\mathfrak{D}}$ in the role of $\mathfrak{P}, \mathfrak{X}_{1}$ in the role of $V$, yields $m\left(\mathfrak{X}_{1}\right) \geqq 2^{w}$. On the other hand, $\mathfrak{B}_{0}$ is a normal subgroup of $\mathfrak{M}_{1}$ of order 2 , so $m(\mathfrak{X}) \geqq 1+2^{w}$.

Let $\mathfrak{F}$ be an elementary subgroup of $\mathfrak{X}$ with $m(\mathfrak{F})=2^{w}+1$, let $\mathfrak{F}_{0}=\mathfrak{F} \cap \mathfrak{S}^{G}$, and let $\mathfrak{F}_{1}$ be a complement to $\mathfrak{F}_{0}$ in $\mathfrak{F}$. Since $m\left(\boldsymbol{E}_{0}\right) \leqq$ $w+2$, we get $m\left(\mathfrak{F}_{1}\right)=a \geqq 2^{w}-1-w$. Since $\mathfrak{F}_{1}$ acts faithfully on $\boldsymbol{O}_{2,2^{\prime}}\left(\mathfrak{M}^{G}\right) / \mathfrak{S}^{G}$, Lemma 5.34 implies that $\boldsymbol{O}_{2,2^{\prime}}\left(\mathfrak{M}^{G}\right) / \mathfrak{S}^{G}$ has a subgroup $\hat{\mathfrak{Q}} / \mathfrak{S}^{G}$ which admits $\mathfrak{F}_{1}$ and such that $\mathfrak{F}_{1} \hat{\mathfrak{Q}} / \mathfrak{S}^{G}$ is the direct product of a dihedral groups of order twice an odd prime. Let $\Re$ be a $S_{2^{\prime}}$-subgroup of $\hat{\mathfrak{\Omega}}$. By Lemma 5.12, $\left[\mathfrak{S}^{G}, \Re\right]=\Re$ is extra special of width $w_{1} \leqq w$. Since $\mathfrak{S}^{G}$ is the central product of $\Omega$ and $C_{\mathfrak{S} G}(\Re)$, and since $\mathfrak{F}_{1} \widehat{\mathscr{\Omega}}=$ $\Re \cdot \boldsymbol{N}_{\mathfrak{C}_{1} \hat{\mathfrak{O}}}(\Re)$, it follows that if $M=\Re / D(\Re)$, then $\boldsymbol{A}_{\mathfrak{C}_{1} \hat{\mathfrak{D}}}(M)$ has a subgroup which is the direct product of $a$ dihedral groups of order twice an odd prime. Let $m(M)=m$. Then $m=2 w_{1} \leqq 2 w$. Since $w \geqq 3$ by hypothesis, we get $w<2^{w}-1-w \leqq a$, and so $2 w<2 a$, whence $m<2 a$. 
This violates Lemma 5.8, and completes the proof.

HYPOTHESIS 7.3. ( i ) $p$ is odd.

(ii) $\mathfrak{B}$ is a $S_{p}$-subgroup of $\mathbb{B}, \mathfrak{A}$ is a normal elementary subgroup of $\mathfrak{P}$ with $m(\mathfrak{U}) \geqq 3, \boldsymbol{Z}(\mathfrak{P})$ is cyclic, and $\boldsymbol{A}_{\mathscr{G}}(\mathscr{C})=\boldsymbol{A}(\mathscr{C})$, where $\mathscr{C}$ : $\mathfrak{A}: \supset \mathfrak{B} \cap Z(\mathfrak{P}) \supset 1$. Also, $\mathfrak{A} \triangleleft N(Z(\mathfrak{P}) \cap \mathfrak{U})$.

LEMMA 7.3. Suppose Hypothesis 7.3 is satisfied. Let $3=\boldsymbol{Z}(\mathfrak{\Re}) \cap$ I. Then each subgroup of $\mathfrak{A}$ of type $(p, p)$ which contains 3 is in $\mathscr{E}(p)$.

Proof. The lemma is an immediate consequence of Lemma 5.5, together with Hypothesis 7.1 (i).

Hypothesis 7.4. (i) (B) is simple.

(ii) $\{2,3\} \cong \pi_{4}(\mathbb{S})$.

(iii) The centralizer of every involution of $\mathbb{S}$ is solvable.

(iv) The normalizer of every nonidentity 3-sugroup of \&S is solvable.

(v) If $\mathfrak{A} \in \mathscr{S}_{\text {cnn }_{3}(2) \cup \mathscr{S}_{\text {cns }_{3}}(3) \text {, then }}$ ( $(\mathfrak{U})$ contains only 1 .

All remaining lemmas in this section are proved under Hypothesis 7.4.

DEFINITION 7.2.

$$
\begin{aligned}
\mathscr{N}=\{(\mathfrak{A}, \mathfrak{B}) \mid 1 . & \mathfrak{A} \text { is a } 2 \text {-subgroup of } \mathbb{S} . \\
2 . & \mathfrak{B} \text { is a } 3 \text {-subgroup of } \mathbb{S} . \\
3 . & \langle\mathfrak{A}, \mathfrak{B}\rangle \text { is not solvable. }\}
\end{aligned}
$$

We remark that in the following lemmas, Lemma 7.1 may be invoked, since Hypothesis 7.4 implies that Hypothesis 7.1 is satisfied for $p=2$ and for $p=3$.

LEMma 7.4. If $\mathfrak{A}$ is a four-subgroup of (S) which centralizes every element of $\mathrm{U}(\mathfrak{U} ; 3)$ and $\mathfrak{B}$ is a subgroup of (S) of type $(3,3)$ which centralizes every element of $И(\mathfrak{B} ; 2)$, then $(\mathfrak{A}, \mathfrak{B}) \in \mathscr{N}$.

Proof. Notice that if $G, H \in \mathfrak{B}$, then the pair $\left(\mathfrak{U}^{G}, \mathfrak{B}^{H}\right)$ satisfies the hypothesis of the lemma.

Suppose the lemma is false and $\mathfrak{A}, \mathfrak{B}$ are chosen so that $\langle\mathfrak{A}, \mathfrak{B}\rangle$ is minimal. It follows as in Lemma 0.10.2 that $\langle\mathfrak{A}, \mathfrak{B}\rangle=\mathfrak{A} \times \mathfrak{B}$. We may then choose $A$ in $\mathfrak{U}^{\#}$ such that $\boldsymbol{E}(A)$ contains an element $\mathfrak{A}_{1}$ of $\mathscr{U}(2)$. Hence, $\left\langle\mathfrak{U}_{1}, \mathfrak{B}\right\rangle$ is solvable. Thus, we may assume that $\mathfrak{A} \in \mathscr{U}(2)$. Let $\mathfrak{R}=N(\mathfrak{R})$. Since $2 \in \pi_{4}$, we have $O_{2^{\prime}}(\mathfrak{R})=1$. This is absurd since 
$\mathfrak{B}$ centralizes $O_{2}(\mathfrak{R})$ and $\mathfrak{R}$ is solvable. The proof is complete.

We set

$$
\begin{aligned}
\mathscr{S}_{1}= & \{G \mid G \in \mathbb{S}, C(G) \text { is solvable. }\} \\
\mathscr{S}_{p}= & \{G \mid G \in \mathbb{S}, C(G) \text { contains an elementary subgroup } \mathbb{F} \text { of } \\
& \text { order } \left.p^{2} \text { which centralizes every element of } И(\mathfrak{F} ; q)\right\}, \\
& p=2,3, q=2,3, p \neq q .
\end{aligned}
$$

We conclude from Lemma 7.4 that

$$
\mathbb{B S}_{1} \cap \mathrm{BS}_{2} \cap \mathrm{BS}_{3}=\varnothing \text {. }
$$

There are some subtle consequence of (7.2).

\section{DEFINITION 7.3.}

$\mathscr{D}=\{\mathfrak{B} \mid 1 . \quad \mathfrak{B}$ is a noncyclic elementary 3-subgroup of $(S)$.

2. Every element of $\mathfrak{B}$ centralizes an element of $\mathscr{C}(3)$.

3. $\mathfrak{B}$ centralizes every abelian subgroup in $\mathbf{h}(\mathfrak{B} ; 2)$.

LemmA 7.5. Suppose $\mathfrak{A} \in \mathscr{U}(2), \mathfrak{B} \in \mathscr{D}$ and $\mathfrak{T}$ is a 2, 3-subgroup of (S) which contains $\langle\mathfrak{A}, \mathfrak{B}\rangle$. Let $\mathfrak{I}_{2}$ be a $S_{2}$-subgroup of $\mathfrak{T}$. Then LSS(S) (see Definition 2.7) contains an element $\mathfrak{M}$ such that

(a) $O_{2^{\prime}}(\mathfrak{M})=1$.

(b) $\boldsymbol{O}_{2}(\mathfrak{M})$ is the central product of $\left[\mathrm{O}_{2}(\mathfrak{M}), \mathfrak{B}\right]$, which is extra special of width $w=2,3$ or 4 , and of $\boldsymbol{C}_{\boldsymbol{O}_{2}(\mathfrak{M})}(\mathfrak{B})$, which is either cyclic or of maximal class $\geqq 3$.

( c ) $\left[\mathrm{O}_{2}(\mathfrak{M}), \mathfrak{B}\right]$ is the central product of $w \mathfrak{B}$-invariant quaternion groups $\mathfrak{\Omega}_{1}, \cdots, \mathfrak{\Omega}_{w}$ whose centralizers in $\mathfrak{B}$ are $w$ distinct subgroups of order 3. In particular, no element of $\mathfrak{B}^{*}$ centralizes any foursubgroup of $\left[\mathrm{O}_{2}(\mathfrak{M}), \mathfrak{B}\right]$. If $w>2$, then $\boldsymbol{C}_{\boldsymbol{O}_{2}(\mathfrak{M})}(\mathfrak{B})=\left[\mathrm{O}_{2}(\mathfrak{M}) \text {, } \mathfrak{B}\right]^{\prime}$ is the center of $\mathrm{O}_{2}(\mathfrak{M})$.

(d) $\mathfrak{I}_{2} \subset \mathfrak{M}$.

(e) $\mathfrak{B} \subset \mathfrak{M}$, and if $\mathfrak{Q}$ is a quaternion subgroup of $\mathfrak{I}_{2}$ which is normalized by $\mathfrak{B}$ but is not centralized by $\mathfrak{B}$, then $\mathfrak{Q} \subset \boldsymbol{O}_{2}(\mathfrak{M})$.

(f ) If $J$ is an involution of $\mathfrak{M} \cap \boldsymbol{C}(\mathfrak{B})$, then $J \in \boldsymbol{O}_{2}(\mathfrak{M})$. If $\mathfrak{M}$ contains a $S_{2}$-subgroup of $C(J)($ e.g., if $C(J)=\mathfrak{I})$, then $C(J) \subseteq \mathfrak{M}$.

(g) M contains a $S_{2}$-subgroup of (S).

Proof. Let $\mathscr{S}$ be the set of 2,3-subgroups of (S) which contain $\left\langle\mathfrak{B}, \mathfrak{T}_{2}\right\rangle$. Choose $\mathfrak{S}$ in $\mathscr{S}$ so that $|\mathfrak{S}|_{2}$ is maximal. Let $\mathfrak{S}_{p}$ be a $S_{p}$ subgroup of $\mathfrak{S}, p=2,3$, chosen so that $\mathfrak{I}_{2} \subseteq \mathfrak{S}_{2}, \mathfrak{B} \subseteq \mathfrak{S}_{3}$. Let $\mathfrak{A}_{1}, \cdots$, $\mathfrak{A}_{m}$ be all the elements of $\mathscr{U}(2)$ in $\mathfrak{S}_{2}$. By Lemma 7.1, each $\mathfrak{A}_{i}$ centralizes $O_{3}(\mathfrak{S})$, so by Lemma $7.4,\left|O_{3}(\mathfrak{S})\right| \leqq 3$. In particular, $\mathfrak{B}$ is not contained in $\boldsymbol{O}_{2}(\subseteq)$ and $\mathfrak{B}$ centralizes $\boldsymbol{O}_{3}(\mathfrak{S})$. Since $\mathfrak{B} \nsubseteq F(\subseteq), \mathfrak{B}$ 
does not centralize $\boldsymbol{O}_{2}(\mathfrak{S})$. Hence, $\boldsymbol{O}_{2}(\mathfrak{S})$ is nonabelian since $\mathfrak{B} \in \mathscr{D}$. Let $\Re=O_{2}(S)^{\prime} \cap 3\left(O_{2}(\mathfrak{S})\right)$, so that $\Re \neq 1$. Let $\Re=N(\Re), \mathbb{S}=C(\Re)$, and observe that $\mathfrak{B} \subseteq \mathfrak{C}$. Since the centralizer of every involution is solvable, $\mathfrak{C}$ is solvable. Let $\mathfrak{S}^{*}$ be a $S_{2}$-subgroup of $\mathfrak{N}$ which contains $\mathfrak{S}_{2}$. Then $\mathfrak{S} \mathfrak{S}_{2}^{*}$ is solvable. By $D_{2,3}$ in $\mathfrak{S} \mathfrak{S}_{2}^{*}$ and maximality of $|\mathfrak{S}|_{2}$, it follows that $\mathfrak{S}_{2}^{*}=\mathfrak{S}_{2}$. Hence, if $\mathfrak{S}_{2}^{* *}$ is a $\mathbf{S}_{2}$-subgroup of (S) containing $\mathfrak{S}_{2}$, then $\mathfrak{S}_{2}$ contains every element of $\mathscr{Q}_{6}\left(\mathfrak{S}_{2}^{* *}\right)$. We may therefore assume that $\mathfrak{U}_{1} \in \mathscr{\mathscr { C }}\left(\mathfrak{S}_{2}^{* *}\right)$.

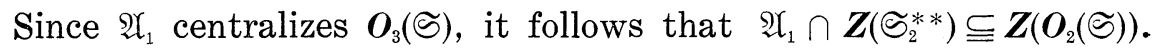
Since $\mathfrak{B}$ centralizes $Z\left(O_{2}(\mathfrak{S})\right)$, maximality of $|\mathfrak{S}|_{2}$ guarantees that $\mathfrak{S}_{2}=$ $\mathfrak{S}_{2}^{* *}$ is a $S_{2}$-subgroup of (S).

Let $\subseteq \subseteq \mathfrak{M} \in \mathscr{L} S(\mathbb{S}$ (S). Thus, (g) holds, as does (d). Since $\mathfrak{M}$ contains a $S_{2}$-subgroup of $(B)$, and since 1 is the only 2-signalizer of (B), it follows that $\boldsymbol{O}_{2^{\prime}}(\mathfrak{M})=1$, and (a) holds. Let $\mathfrak{K}=\boldsymbol{O}_{2}(\mathfrak{M})$. Suppose $\mathfrak{S}$ contains a noncyclic characteristic abelian subgroup $\mathfrak{S}_{0}$. Then $\mathfrak{B}$ centralizes $\mathfrak{S}_{0} \boldsymbol{Z}\left(\mathfrak{S}_{\mathfrak{C}}\right)$ and $\mathfrak{S}_{0} \boldsymbol{Z}\left(\mathfrak{S}_{\mathfrak{C}}\right)$ contains an element of $\mathscr{\mathscr { C }}\left(\mathfrak{S}_{2}\right)$. This violates Lemma 7.4 .

Clearly, $\mathfrak{S}$ is noncyclic, since $\mathfrak{K}=\boldsymbol{F}(\mathfrak{M})$ and $\mathfrak{M}$ is solvable. Thus, $\mathfrak{S C}$ is of symplectic type. The width $w$ of $\mathfrak{F}$ is at least 2 , since $\mathfrak{B}$ is faithfully represented on $\mathfrak{S}$.

Suppose $w \geqq 3$ and $B \in \mathfrak{B}^{\sharp}$ centralizes a four-subgroup $\mathfrak{B}$ of $\mathfrak{S}$ with $\Omega_{1}(\boldsymbol{Z}(\mathfrak{S})) \subset \mathfrak{B}$. By Lemma 7.2, $\mathfrak{B}$ centralizes every element of $\boldsymbol{И}\left(\mathfrak{B} ; 2^{\prime}\right)$. Since $C(B)$ contains an element of $\mathscr{U}(3),(7.2)$ is violated. Thus, if $w \geqq 3$, then no element of $\mathfrak{B}^{\sharp}$ centralizes any four-subgroup of $\mathfrak{K}$. This immediately implies that $\mathfrak{S}$ is extra special and $w \leqq 4$. Now (b) and (c) follow from Lemma 5.12.

We next prove the first assertion of (f). Let $J$ be an involution of $\mathfrak{M} \cap \boldsymbol{C}(\mathfrak{B})$. If $w=2$, then $\mathfrak{B}$ is a $S_{2^{\prime}}$-subgroup of $\mathfrak{M}$ and (f) is clear. Suppose $w \geqq 3$. In this case, $\mathfrak{S}$ is extra special, so $\mathfrak{S} \cap C(\mathfrak{B})=\mathfrak{F C}^{\prime}$ is of order 2. Let $\mathfrak{B}_{0}$ be any subgroup of $\mathfrak{B}$ of order 3 . Since $J$ centralizes $\mathfrak{B}_{0}$, it follows that $J$ normalizes $C_{\mathfrak{H}}\left(\mathfrak{B}_{0}\right)$. We will show that $J$ centralizes $C_{\mathfrak{5}}\left(\mathfrak{B}_{0}\right)$. This is clear if $\boldsymbol{C}_{\mathfrak{g}}\left(\mathfrak{B}_{0}\right)=\mathfrak{S E}^{\prime}$, so suppose $\boldsymbol{C}_{\mathfrak{g}}\left(\mathfrak{B}_{0}\right) \supset \mathfrak{S C}^{\prime}$. Since $\mathfrak{F}$ is extra special, so is $C_{\mathfrak{H}}\left(\mathfrak{B}_{0}\right)$, so $C_{\mathfrak{H}}\left(\mathfrak{B}_{0}\right)$ is a quaternion group on which $\mathfrak{B} / \mathfrak{B}_{0}$ is faithfully represented. Since a quaternion group has no automorphism of order $6, J$ necessarily centralizes $C_{\mathfrak{S}_{\mathfrak{T}}}\left(\mathfrak{B}_{0}\right)$. Hence, $J$ centralizes $\left\langle C_{\mathfrak{S}}\left(\mathfrak{B}_{0}\right)\left|\mathfrak{B}_{0} \subset \mathfrak{B},\right| \mathfrak{B}_{0} \mid=3\right\rangle=\mathfrak{F}$, so $J \in \mathfrak{S}_{\mathcal{C}}$. This proves the first assertion of (f).

Now for the second assertion of (f). If $w>2$, then $\langle J\rangle=\mathfrak{S g}^{\prime}$, by what we have just shown, together with (c). So suppose $w=2$ and $\langle J\rangle \rtimes \mathfrak{M}$. Let $\mathfrak{S}_{0}=\left[\mathfrak{S}_{\mathfrak{E}}, \mathfrak{B}\right], \mathfrak{S}_{1}=C_{\mathfrak{S}}(\mathfrak{B})$. Thus, $J \in \mathfrak{S}_{1}, J \neq Z$, where $Z$ is central involution of $\mathfrak{S}_{1}$. Since $w=2$, $\mathfrak{B}$ is a $S_{2^{\prime}}$-subgroup of $\mathfrak{M}$. Let $\mathfrak{I}_{0}$ be a $S_{2}$-subgroup of $C(J)$ which is contained in $\mathfrak{M}$. Thus, $C(J) \supseteqq \mathfrak{I}_{0} \mathfrak{B}, \mathfrak{I}_{0} \supseteqq \mathfrak{F}_{0} \times\langle J\rangle$, and $\mathfrak{S}_{0}$ is the central product of 2 quaternion groups. 
Since $C(J)$ contains an element of $\mathscr{U}(2)$, it follows that $\boldsymbol{O}_{2^{\prime}}(C(J))=$ 1. Since $Z$ centralizes $\mathfrak{I}_{0}$, a $S_{2}$-subgroup of $C(J)$, it follows that $Z \in \boldsymbol{O}_{2}(C(J))$, and so $Z \in Z\left(\boldsymbol{O}_{2}(C(J))\right)$. Since $\mathfrak{B} \subseteq C(J)$, it follows that $\boldsymbol{O}_{2}(\boldsymbol{C}(J)) \in \boldsymbol{U}_{\mathfrak{M}}(\mathfrak{B} ; 2)$. Hence, $\boldsymbol{O}_{2}(\boldsymbol{C}(J)) \subseteq \boldsymbol{O}_{2}(\mathfrak{M})=\mathfrak{F}$, since $\mathfrak{B}$ is a $S_{2^{\prime}}$ subgroup of $\mathfrak{M}$. Hence, $\boldsymbol{O}_{2}(C(J)) \subseteq \mathfrak{S}_{\mathcal{C}} \cap \mathfrak{I}_{0}=\mathfrak{S}_{0} \times\langle J\rangle$. If $\boldsymbol{O}_{2}(C(J))$ is not elementary, then $\langle Z\rangle$ char $\boldsymbol{O}_{2}(C(J))$, and so $C(J) \cong \mathfrak{M}$. Suppose $\boldsymbol{O}_{2}(\boldsymbol{C}(J))$ is elementary. Since $\mathfrak{B}$ is faithfully represented on $\boldsymbol{O}_{2}(\boldsymbol{C}(J))$, it follows that $\left|\boldsymbol{O}_{2}(C(J))\right| \geqq 2^{4}$. However, $\boldsymbol{O}_{2}(\mathfrak{M})$ contains no elementary subgroup of order $2^{4}$ on which $\mathfrak{B}$ is faithfully represented. This completes the proof of the second assertion of (f).

We turn to the proof of (e). Let $\cong$ be a quaternion subgroup of $\mathfrak{M}$ normalized but not centralized by $\mathfrak{B}$. Let $\mathfrak{B}_{0}=\mathfrak{B} \cap C(\mathfrak{Q})$, so that $\mathfrak{B} \mathfrak{Q}=\mathfrak{B}_{0} \times \mathfrak{B}_{1} \mathfrak{Q}$, where $\left|\mathfrak{B}_{i}\right|=3$ and $\mathfrak{B}_{1}$ is faithfully represented on $\mathfrak{Q}$. Let $\mathfrak{\Omega}_{0}=\mathfrak{Q} \cap \mathfrak{K}$. By (f), $\mathfrak{\Omega}_{0} \supseteq \mathfrak{Q}^{\prime}$. Since $\mathfrak{Q} / \mathfrak{\Omega}^{\prime}$ is an irreducible $\mathfrak{B}$ group, we may assume by way of contradiction that $\mathfrak{\Omega}_{0}=\mathfrak{\Omega}^{\prime}$.

Let $\mathfrak{R}$ be a $\mathfrak{B \Omega}$-invariant subgroup of $\boldsymbol{Q}_{2}^{\prime}(\mathfrak{M})$ minimal subject to $[\Re, \mathfrak{Q}] \neq 1$. Thus, $\Re$ may be viewed as a $\mathfrak{B} \mathfrak{Q} / \mathfrak{Q}^{\prime}$-group; as such $\mathfrak{B}_{1} \mathfrak{Q} / \mathfrak{\mathfrak { Q } ^ { \prime }}$ acts faithfully. Since $w \leqq 4$, it follows that $\Re$ is elementary of order $3^{3}$ and is centralized by $\mathfrak{B}_{0}$. Thus, $w=4$ and $S_{3}$-subgroups of $\mathfrak{M}$ are of order $3^{5}$. Also, $\Re$ is incident with an elementary subgroup $\Re_{0}$ of $\mathfrak{M}$ such that $\left\langle\mathfrak{B}_{0}, \mathfrak{R}_{0}\right\rangle$ is elementary of order $3^{4}$.

Let $\mathfrak{P}$ be a $S_{3}$-subgroup of $\mathbb{B}$ containing $\left\langle\mathfrak{B}_{0}, \mathfrak{R}_{0}\right\rangle$ and choose $\mathfrak{U}$ in $\mathscr{C}(\mathfrak{P})$. Then $\left\langle\mathfrak{B}_{0}, \Re_{0}\right\rangle$ contains an elementary subgroup $\mathbb{F}$ of order $3^{3}$ which centralizes $\mathfrak{H}$. Since $\mathfrak{F} \subseteq \mathbb{M}$, there is an element $E$ of $\mathfrak{F}$ such that $\mathfrak{F} \cap C(E)$ contains a four-group. But then $E \in \mathcal{S}_{1} \cap \mathcal{S}_{2} \cap \mathfrak{S}_{3}$, against (7.2). This contradiction completes the proof of (e) and the lemma.

Throughout the remainder of this section, $\mathfrak{F}$ denotes a $S_{3}$-subgroup of (3).

Lemma 7.6. Suppose $|\mathfrak{P}|>3^{4}$.

(a) If $\mathfrak{P}_{0}$ is a subgroup of $\mathfrak{P}$ of index at most 9 and $\mathfrak{P}_{0}$ contains an element of $\mathscr{U}^{*}(\mathfrak{P})$, then $\mathrm{U}\left(\mathfrak{\Re}_{0} ; 2\right)$ contains only 1.

(b) If $\mathfrak{A}$ is a subgroup of $\mathfrak{P}$ of type $(3,3)$ and $\left|\mathfrak{P}: \boldsymbol{C}_{\mathfrak{P}}(\mathfrak{H})\right| \leqq 3$, then $\mathfrak{A}$ centralizes every element of $\mathrm{U}(\mathfrak{H} ; 2)$.

( c ) If $\mathfrak{A}$ is a subgroup of $\mathfrak{P}$ of type $(3,3)$, if $\left|\mathfrak{P}: C_{\mathfrak{P}}(\mathfrak{Q})\right| \leqq 9$, and if $C_{\mathfrak{P}}(\mathfrak{U})$ contains an element of $\mathscr{U}^{*}(\mathfrak{P})$, then $\mathfrak{U} \in \mathscr{D}$.

(d) If $\mathbb{F}$ is a normal elementary subgroup of $\mathfrak{B}$ of order 27 and $\left|\mathfrak{P}: \boldsymbol{C}_{\mathfrak{P}}(\mathfrak{\xi})\right|=3$, then $\mathfrak{U} \in \mathscr{E}(3)$ for each subgroup $\mathfrak{U}$ of index 3 in $\mathfrak{\xi}$.

Proof. (a) Let $\mathfrak{B}$ be an element of $\mathscr{C}^{*}(\mathfrak{P})$ with $\mathfrak{B} \subseteq \mathfrak{P}_{0}$. We will show that $И(\mathfrak{B} ; 2)$ contains only 1 . To do this, we first show that if $\mathfrak{X} \in \mathscr{Q}(3)$, then $|\boldsymbol{C}(\mathfrak{X})|$ is odd. Suppose $J$ is an involution of of $C(\mathfrak{X})$. By Lemma 5.38, $C(J)$ contains an element $\mathfrak{Y}$ of $\mathscr{U}(2)$. Hence, 
by Lemmas 7.1 and $7.4,\langle\mathfrak{X}, \mathfrak{Y}\rangle$ is nonsolvable, against $\langle\mathfrak{X}, \mathfrak{Y}\rangle \leqq C(J)$. In particular, $\mathfrak{X}$ does not centralize any quaternion subgroup of $\mathbb{( S S}$. By Lemma 7.1(iii), it follows that $\mathfrak{B}$ centralizes every element of $И(\mathfrak{B} ; 2)$. Suppose $K$ is an involution of $C(\mathfrak{B})$. Then by Lemma $5.38, C(\Re)$ contains an element 3 of $\mathscr{U}(2)$, so by Lemmas 7.1 and $7.4,\langle\mathfrak{B}, 3\rangle$ is nonsolvable, against $\langle\mathfrak{B}, \mathfrak{B}\rangle \subseteq C(K)$. We conclude that $|\boldsymbol{C}(\mathfrak{B})|$ is odd, and so $U(\mathfrak{B} ; 2)$ contains only 1 . Since $U\left(\mathfrak{P}_{0} ; 2\right) \subseteq И(\mathfrak{B} ; 2)$, (a) follows.

Suppose (b) is false. Let $\mathfrak{Q}$ be a 2 -group normalized by $\mathfrak{A}$ and minimal subject to $[\mathfrak{Q}, \mathfrak{X}] \neq 1$. Then $\mathfrak{Q}=[\mathfrak{Q}, \mathfrak{X}]$ is either a quaternion group or a four-group, and $\mathfrak{A}=\mathfrak{A}_{0} \times \mathfrak{U}_{1}$ where $\left|\mathfrak{A}_{i}\right|=3$ and $\mathfrak{A}_{0}=\boldsymbol{C}_{\mathfrak{Q}}(\mathfrak{Q})$.

Let $\mathfrak{S}=\boldsymbol{C}\left(\mathfrak{U}_{0}\right) \supseteqq\left\langle\boldsymbol{C}_{\mathfrak{P}}(\mathfrak{U}), \mathfrak{Q}\right\rangle$. Since $\boldsymbol{C}_{\mathfrak{\beta}}(\mathfrak{U})$ is of index at most 3 in $\mathfrak{P}$, it follows that $\boldsymbol{C}_{\mathfrak{P}}(\mathfrak{H})$ contains an element $\mathfrak{U}$ of $\mathscr{U}(\mathfrak{P})$. We argue that $C_{\mathfrak{P}}(\mathfrak{U})$ contains an element of $\mathscr{S}_{\text {cnn }_{3}}(\mathfrak{B})$. Namely, let

$$
3=\Omega_{1}\left(\boldsymbol{Z}\left(\boldsymbol{C}_{\mathfrak{\beta}}(\mathfrak{Q})\right)\right) \text {. }
$$

If $m(\mathfrak{B}) \geqq 3$, let $\mathfrak{B}$ be an element of $\mathscr{S}_{\text {cne }}(\mathfrak{P})$ which contains 3 . Since $\mathfrak{U} \subseteq \mathfrak{B}$, we get $\mathfrak{B} \subseteq \boldsymbol{C}_{\mathfrak{P}}(\mathfrak{H})$. Suppose $m(\mathfrak{Z}) \leqq 2$. Then $3=\mathfrak{A} \triangleleft \mathfrak{P}$, so by Lemma $0.8 .9, \mathfrak{A}$ is contained in some element of $\mathscr{S}_{\text {cnn }}(\mathfrak{P})$. So $\boldsymbol{C}_{\mathfrak{\beta}}(\mathfrak{U})$ contains an element of $\mathscr{S}_{\text {sn }_{3}(\mathfrak{P})}$. By Hypothesis 7.4(v), $\boldsymbol{O}_{3^{\prime}}(\mathfrak{C})=1$.

Let $\mathfrak{P}^{*}$ be a $S_{3}$-subgroup of $C$ which contains $C_{\mathfrak{P}}(\mathfrak{U})$. Since $\mathfrak{U}_{1}$ does not centralize $\boldsymbol{O}_{3}(\mathfrak{S})=\mathscr{S}$, it follows that $\mathfrak{P}^{*}=\mathfrak{S}_{\mathfrak{S}} \boldsymbol{C}_{\mathfrak{P}}(\mathfrak{H})$ is a $S_{3^{-}}$ subgroup of $\mathfrak{S}$. Also, since $\mathfrak{A}_{1} \mathfrak{E} / \mathfrak{C} \subseteq Z\left(\mathfrak{F}^{*} / \mathfrak{C}\right)$, it follows that $\mathfrak{A}_{1} \subseteq$ $\boldsymbol{O}_{3,3^{\prime}, 3}(\mathfrak{C})$. Hence, $\mathfrak{\varrho} \subseteq \boldsymbol{O}_{3,3^{\prime}}(\mathfrak{E})$. Since $\boldsymbol{C}_{\mathfrak{5}}\left(\mathfrak{U}_{1}\right)$ is of index 3 in $\mathfrak{S}$, it follows that $\left[\boldsymbol{Q}_{3}^{1}(\mathfrak{E}), \mathfrak{A}_{1}\right]$ is a quaternion group. Hence, $\widetilde{\mathfrak{S}}=\mathfrak{\Omega}^{*}$ is a group.

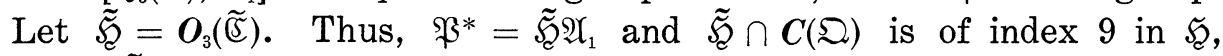
while $\tilde{\mathfrak{B}} \cap \boldsymbol{C}(\mathfrak{Q}) \triangleleft \mathfrak{P}^{*}$. Since $\mathfrak{\Omega}^{\prime}$ centralizes no element of $\mathscr{U}^{*}\left(\mathfrak{P}^{*}\right)$, it follows that $\tilde{\mathscr{B}} \cap C(\mathfrak{C})$ is cyclic. Since $|\mathfrak{B}|>3^{4}$, so also $\left|\mathfrak{P}^{*}\right|>3^{4}$, so $\mathfrak{A}_{0}$ is a proper subgroup of $\tilde{\mathfrak{S}} \cap \boldsymbol{C}(\mathfrak{Q})=\widetilde{\mathfrak{A}}_{0}$.

Let $\tilde{\mathfrak{F}}_{0}=[\mathfrak{\Omega}, \widetilde{\mathfrak{F}}]$. By the three subgroups lemma, $\tilde{\mathfrak{F}}_{0}$ and $\tilde{\mathfrak{A}}_{0}$ commute elementwise. Furthermore, either $\tilde{\mathfrak{B}}_{0}$ is elementary of order 9 and $\tilde{\mathfrak{S}}=\tilde{\mathfrak{S}}_{0} \times \tilde{\mathfrak{A}}_{0}$ or $\tilde{\mathfrak{S}}_{0}$ is a non abelian group of order 27 and exponent 3 and $\tilde{\mathfrak{F}}$ is the central product of $\tilde{\mathfrak{K}}_{0}$ and $\tilde{\mathfrak{A}}_{0}$.

Since $C\left(\mathfrak{U}_{1}\right) \cap \tilde{\mathcal{Y}}$ is of index 3 in $\tilde{\mathcal{H}}$, it follows that $\mathfrak{A}_{1}$ centralizes $\tilde{\mathfrak{U}}_{0}$. Set $\mathfrak{B}=\left\langle\mathfrak{A}_{1}, \tilde{\mathfrak{A}}_{0}\right\rangle=\mathfrak{A}_{1} \times \tilde{\mathfrak{A}}_{0}$, and let $I$ be the involution of $\mathfrak{Q}$. Thus, $\mathfrak{B} \subseteq C(I)$ and $C(I)$ contains an element of $\mathscr{C}(2)$. Thus, $C(I)$ contains no element of $\mathscr{C}^{*}(3)$. Since $\mathfrak{B}$ is of index 9 in $\mathfrak{P}^{*}$, it follows that $\mathfrak{B}$ is a $S_{3}$-subgroup of $C(I)$. Let $\mathbb{2}$ be a $S_{2,3}$-subgroup of $C(I)$ which contains $\mathfrak{B} \mathfrak{O}$. Then $O_{3}(\mathfrak{R})=1$, so $\mathfrak{B}$ is faithfully represented on $\boldsymbol{O}_{2}(\mathfrak{R})$. We can thus choose a subgroup $\mathfrak{B}_{0}$ of order 3 in $\mathfrak{B}$ such that $\tilde{\mathfrak{U}}_{0}$ is faithfully represented on $\boldsymbol{O}_{2}(\mathfrak{L}) \cap \boldsymbol{C}\left(\mathfrak{B}_{0}\right)$. Let $\mathfrak{X}=\boldsymbol{C}\left(\mathfrak{B}_{0}\right)$. Then $\boldsymbol{O}_{3^{\prime}}(\mathfrak{X})$ is of odd order by (a). Thus, $\boldsymbol{O}_{3^{\prime}, 3}(\mathfrak{X}) \cap \tilde{\mathfrak{A}}_{0}=1$, so that $\left|\boldsymbol{O}_{3^{\prime}, 3}(\mathfrak{X})\right|_{3} \leqq$ 27. But $\tilde{\mathfrak{A}}_{0}$ is faithfully represented on the Frattini quotient group $\mathfrak{B}$

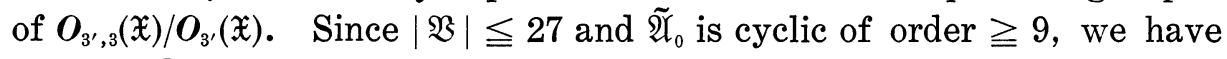
a contradiction. The proof of (b) is complete. 
Suppose (c) is false. Let $\mathfrak{B}$ be a four group in $И(\mathfrak{H})$ which is not centralized by $\mathfrak{A}$. Then $\mathfrak{A}=\mathfrak{A}_{0} \times \mathfrak{A}_{1}$ where $\left|\mathfrak{A}_{i}\right|=3$ and $\mathfrak{A}_{0}=C_{\mathfrak{A}}(\mathfrak{B})$.

Set $\mathfrak{C}=\boldsymbol{C}\left(\mathfrak{A}_{0}\right)$ and let $\mathfrak{P}^{*}$ be a $S_{3}$-subgroup of $\mathbb{E}$ which contains $\boldsymbol{C}_{\mathfrak{P}}(\mathfrak{H})$. By (a), $\boldsymbol{O}_{3^{\prime}}(\mathfrak{E})$ is of odd order, so $\mathfrak{A}_{1} \mathfrak{B}$ is faithfully represented on $O_{3^{\prime}, 3}(\mathfrak{E}) / O_{3^{\prime}}(\mathfrak{E})$. Set $\mathfrak{S}=\mathfrak{P}^{*} \cap O_{3^{\prime}, 3}(\mathfrak{E})$. By $(B),\left|\mathfrak{P}: C_{\mathfrak{h}}\left(\mathfrak{A}_{1}\right)\right| \geqq 9$. Thus, $\mathfrak{P}^{*}=\mathfrak{S} C_{\mathfrak{P}_{3}}(\mathfrak{U})$ is a $S_{3^{-}}$subgroup of $\mathbb{S}$, so that $\boldsymbol{O}_{3^{\prime}}(\mathfrak{E})=1$.

We may now apply Lemma 5.42 with $\mathfrak{S} / \mathfrak{S}$ in the role of $\mathfrak{S}, \mathfrak{S} / D(\mathfrak{S})$ in the role of $\mathfrak{F}$, and $\mathfrak{A}_{1}$ in the role of 3 . Let $\mathfrak{F}_{1}$ be the inverse image in $\mathfrak{E}$ of $\left[\boldsymbol{Q}_{3}^{1}(\mathfrak{E}), \mathfrak{A}_{1}\right]$. Thus, $\mathfrak{E}_{1}=\mathfrak{S} \mathfrak{O}$ where $\mathfrak{Q}$ is either a four-group or is the central product of 2 quaternion groups. Since $C_{\mathfrak{B}}\left(\mathfrak{U}_{1}\right)$ covers $\mathfrak{P}^{*} / \mathfrak{S}$, it follows that $\mathfrak{S}_{1}$ is a minimal subgroup of the group $\Omega=\mathfrak{B}_{1} \mathfrak{P}^{*}$. Let $\mathbb{Z}=\boldsymbol{O}_{3}(\Re)$, so that $\mathfrak{B}^{*} / \mathbb{R}$ is elementary of order 3 or 9 . Since $\mathfrak{B} \subset \mathfrak{C}_{1}$, we assume without loss of generality that $\mathfrak{B} \subseteq \mathfrak{D}$.

Since $\mathfrak{A}_{1}$ centralizes $\boldsymbol{D}(\mathfrak{Q})$, so does $\mathfrak{\Omega}$. Thus, $\boldsymbol{C}(\mathfrak{Q}) \cap \mathfrak{Q} \triangleleft \mathbb{Q}$. Since

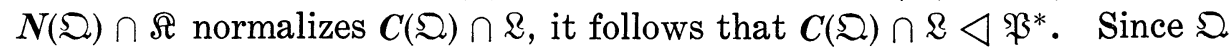
centralizes no element of $\mathscr{C}^{*}(3)$, it follows that $C(\mathfrak{Q}) \cap \mathbb{R}$ is cyclic. Naturally, $\mathfrak{A}_{0} \subseteq C(\Omega) \cap \mathbb{R}$.

Case 1. $\mathfrak{P}^{*}=\mathfrak{R N}_{1}$.

Since $\mathfrak{A}_{1}$ normalizes $\mathfrak{B}$, it follows that $\mathfrak{R}_{1}=\mathfrak{P} * \mathfrak{B}$ is a group and

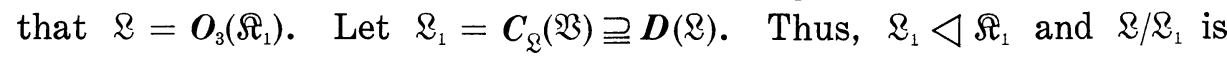
elementary of order 27. Also, $\mathbb{R}_{1}$ is cyclic, since no element of $\mathscr{C}^{*}(3)$ is centralized by $\mathfrak{B}$. Since $\mathscr{R} / \mathfrak{R}_{1}$ is a chief factor of $\Re_{1}$ or order 27 , it follows that $\mathbb{R}=\mathfrak{R}_{1} \times \mathbb{R}_{2}$, where $\mathfrak{Z}_{2}=[\mathbb{R}, \mathfrak{B}]$ is elementary of order 27 , $\mathfrak{R}_{2} \triangleleft \mathfrak{R}_{1}$. Let $V$ be an involution of $\mathfrak{B}$. Thus, $\mathfrak{B}=\left\langle C(V) \cap \mathfrak{R}_{2}, \mathfrak{A}_{0}\right\rangle$ is elementary of order 9 and $\left|\mathfrak{P}^{*}: \boldsymbol{C}_{\mathfrak{F}^{*}}(\mathfrak{B})\right|=3$.

By $(b), \mathfrak{B}$ centralizes every element of $\boldsymbol{U}(\mathfrak{B} ; 2)$. Since $C(V)$ contains an element of $\mathscr{U}(2),(7.2)$ is violated.

Case 2. $\mathfrak{P}^{*} \supset \mathfrak{R A}_{1}$.

In this case, $\mathfrak{P}^{*} / \mathbb{R}$ is elementary of order 9 , so $\mathfrak{Q}$ is the central product of 2 quaternion groups.

Suppose $\mathbb{R}$ is abelian. Then $\mathbb{L}=\mathfrak{Z}_{1} \times \mathbb{Z}_{2}$ where $\mathfrak{Z}_{1}=[\mathbb{R}, \mathfrak{\Omega}]$ is elementary of order $3^{4}$ and $\mathbb{\Omega}_{2}=C_{\Omega}(\mathfrak{Q})$ is cyclic. Notice that $\mathfrak{A}_{0} \subseteq \Omega_{2}$. Since $C_{\mathfrak{Q}}(\mathfrak{B})$ is of index 27 in $\&$ by $(B)$, it follows that $\mathbb{C} \cap\left(\mathfrak{U}_{1}\right) \cap C(\mathfrak{B})$ contains a subgroup $\mathfrak{B}$ of type $(3,3)$. But then $\left|\mathfrak{P}^{*}: C_{\mathfrak{B}^{*}}(\mathfrak{B})\right| \leqq 3$, so by (b), $\mathfrak{B}$ centralizes every element of $И(\mathfrak{B} ; 2)$. Hence, $\mathfrak{B}^{\sharp} \cong \mathbb{S}_{1} \cap \mathfrak{S}_{2} \cap \mathfrak{S}_{3}$, against (7.2). We conclude that $\mathbb{Q}$ is non abelian.

Since $\mathfrak{U}_{1}$ centralizes $\boldsymbol{D}(\mathbb{Q})$, so does $\mathfrak{Q}$, so $\mathfrak{\Omega}_{2}=\boldsymbol{C}_{\mathbb{\Omega}}(\mathfrak{Q}) \triangleleft \mathbb{\Omega}$. Hence $\mathfrak{Q}_{2} \triangleleft \mathfrak{P}^{*}$, and $\mathfrak{Z}_{2}$ is cyclic. Let $\mathfrak{\Omega}_{1}=\left[\mathbb{\Omega}, \mathfrak{\Omega}\right.$. Then $\mathfrak{Q}_{1} / \boldsymbol{D}\left(\mathfrak{R}_{1}\right)$ is elementary of order $3^{4}, \mathbb{R}_{1}^{\prime}=\boldsymbol{D}\left(\mathbb{R}_{1}\right)$ and $\mathbb{\Omega}_{1} / \boldsymbol{D}\left(\mathbb{\Omega}_{1}\right)$ is a chief factor of $K$. Being a

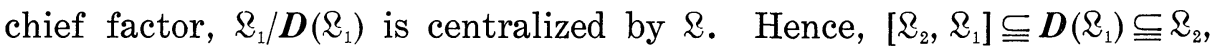
so $\left[\mathfrak{R}_{2}, \mathfrak{R}_{1}, \mathfrak{\Omega}\right]=1$. Since $\left[\mathfrak{Q}, \mathfrak{R}_{2}\right]=1$, so also $\left[\mathfrak{Q}, \mathfrak{R}_{2}, \mathfrak{R}_{1}\right]=1$. By the three subgroups lemma, $\left[\mathfrak{Q}_{1}, \mathfrak{\Omega}, \mathfrak{\Omega}_{2}\right]=1$, that is, $\left[\mathfrak{Q}_{1}, \mathfrak{\Omega}_{2}\right]=1$. Hence, 
$\boldsymbol{D}\left(\mathfrak{R}_{1}\right) \subseteq \boldsymbol{Z}\left(\mathfrak{R}_{1}\right)$. Since $\mathbb{R}$ is nonabelian, so is $\mathfrak{R}_{1}$. Since $\mathfrak{R}_{1} / \boldsymbol{D}\left(\mathbb{R}_{1}\right)$ is a chief factor of $\Re, D\left(\mathfrak{Q}_{1}\right)=\boldsymbol{Z}\left(\mathfrak{Q}_{1}\right)=\mathfrak{Q}_{1}^{\prime} \subseteq \mathfrak{\Omega}_{2}$, so $\mathfrak{Q}_{1}$ is extra special of order $3^{5}$.

Now $\mathfrak{Y}_{1} \mathfrak{B}$ is faithfully represented on $\mathbb{R}_{1}$. Also, $|\mathfrak{L}: \mathbb{R} \cap C(\mathfrak{B})|=$ $\left|\mathfrak{Q}_{1}: \mathfrak{Q}_{1} \cap \boldsymbol{C}(\mathfrak{B})\right|=3^{3}$, by $(B)$. Hence, $\mathfrak{Q}_{1} \cap C(\mathfrak{B})=3^{2}$. This is not the case, since $\mathfrak{R}_{1} \cap \boldsymbol{C}(\mathfrak{B})$ is either extra special or is $\mathfrak{R}_{1}^{\prime}$. The proof of (c) is complete.

Suppose $(d)$ is false. Let $\mathfrak{Z}$ be an element of $И\left(\mathfrak{A} ; 3^{\prime}\right)$ minimal subject to $[\mathfrak{U}, \mathfrak{Q} \neq \neq 1$. Then $\mathfrak{Q}$ is a $q$-group for some prime $q, \mathfrak{\Omega}=$ $[\mathfrak{Q}, \mathfrak{A}]$, and $\mathfrak{A}=\mathfrak{A}_{0} \times \mathfrak{A}_{1}$, where $\left|\mathfrak{A}_{i}\right|=3$ and $\mathfrak{A}_{0}=\boldsymbol{C}_{\mathfrak{Q}}(\mathfrak{Q})$. By (b), $q \neq 2$. Let $\mathfrak{E}=\boldsymbol{C}\left(\mathfrak{U}_{0}\right)$. Since $\boldsymbol{C}_{\mathfrak{S}}(\mathfrak{U})$ contains an element of $\mathscr{S} \mathscr{C} \mathscr{N}_{3}(\mathfrak{P})$, it follows from Hypothesis $7.4(\mathrm{v})$ that $O_{3^{\prime}}(\mathfrak{S})=1$. Let $\mathfrak{S}^{*}$ be a $S_{3^{-}}$ subgroup of $\sqrt{5}$ which contains $C_{\mathfrak{B}}\left(\mathfrak{A}_{0}\right)$. Since $\left|\mathfrak{P}: \boldsymbol{C}_{\mathfrak{B}}(\mathfrak{A})\right| \leqq 3$, so also $\left|\mathfrak{P}^{*}: \boldsymbol{C}_{\mathfrak{P}}\left(\mathfrak{U}_{0}\right)\right| \leqq 3$, and so $\left[\mathfrak{P}^{*}, \mathfrak{A}, \mathfrak{A}\right]=1$.

Let $\mathfrak{S}_{\mathfrak{z}}$ be a $S_{3, q}$-subgroup of $\left(5\right.$ which contains $\mathfrak{P}^{*}$. Since $q$ is odd, (B) implies that $\mathfrak{U} \subseteq O_{3}(\mathfrak{S})$. Let $\mathfrak{S}^{*}$ be a $S_{3, q}$-subgroup of $\sqrt{5}$ which contains $\mathfrak{X} \cong$. By Lemma 0.7.5, we get $\mathfrak{U} \subseteq \boldsymbol{O}_{3}\left(\mathfrak{S}^{*}\right)$, so $\mathfrak{Q}=[\mathfrak{Q}, \mathfrak{X}] \subseteq$ $\boldsymbol{O}_{3}\left(\mathfrak{S}^{*}\right)$. This contradiction completes the proof of $(\mathrm{d})$ and the lemma.

Lemma 7.7. Assume the following:

(a) $\mathfrak{W}$ is a normal elementary subgroup of $\mathfrak{P}, \mathfrak{U}=\boldsymbol{A}_{\mathscr{S}}(\mathfrak{W})$.

(b) $\overline{\mathfrak{B}}$ is the image of $\mathfrak{B}$ in $\mathfrak{A}$ and $\overline{\mathfrak{B}}$ is faithfully represented on $\cong, \cong$ being a non abelian special 2-subgroup of $\mathfrak{A}$.

(c) $\overline{\mathfrak{P}}$ contains a subgroup $\overline{\mathfrak{P}}_{0}$ of order 3 which centralizes a hyperplane of $\mathfrak{M}$.

Then $\overline{\mathfrak{P}}$ centralizes $\mathfrak{Q}$.

Proof. Let $\mathfrak{\Omega}_{0}=\left[\overline{\mathfrak{P}}_{0}, \mathfrak{\Omega}\right]$. Thus, $\mathfrak{\Omega}_{0}$ is a quaternion group, and $\mathfrak{W}=\mathfrak{W}_{0} \times \mathfrak{W}_{1}$, where $\mathfrak{W}_{0}=\left[\mathfrak{\Omega}_{0}, \mathfrak{W}\right]$ is of order 9 and $\mathfrak{W}_{1}=\boldsymbol{C}_{\mathfrak{M}_{3}}\left(\mathfrak{\Omega}_{0}\right)$. Since $|C(\mathfrak{W})|$ is odd, some involution $I$ of $N(\mathfrak{W})$ maps to the involution of $\mathfrak{\Omega}_{0}$. Let $\overline{\mathfrak{P}}_{1}$ be the normal closure of $\overline{\mathfrak{P}}_{0}$ in $\overline{\mathfrak{P}}$. Thus, $\overline{\mathfrak{P}}_{1}$ centralizes $\mathfrak{\Omega}^{\prime}$. Let $\mathfrak{W}_{2}=C_{\mathfrak{W}}\left(\overline{\mathfrak{P}}_{1}\right)$ so that $\mathfrak{\Omega}^{\prime}$ is faithfully represented on $\mathfrak{W}_{2}$. Suppose $\overline{\mathfrak{P}}$ does not centralize $\mathfrak{\Omega}$. Then by Lemma 4.4 of [17], there is an elementary subgroup $\mathfrak{W}^{*}$ of $\mathfrak{W}_{2}$ which is of order 27 , normal in $\mathfrak{P}$ and with $\left|\mathfrak{P}: \boldsymbol{C}_{\mathfrak{B}}\left(\mathfrak{W}^{*}\right)\right|=3$. Since $\overline{\mathfrak{P}}_{0}$ centralizes $\mathfrak{W}^{*}$, it follows that $\mathfrak{W}^{*} \cap \mathfrak{W}_{1}$ is noncyclic. Let $\mathfrak{B}$ be a subgroup of $\mathfrak{W}^{*} \cap \mathfrak{W}_{1}$ of order 9 . With $\mathfrak{W}^{*}$ in the role of $\mathfrak{F}$ in Lemma $7.6(\mathrm{~d})$, we conclude that $\mathfrak{B} \in \mathscr{E}(3)$. But now $C(I)$ contains an element of $\mathscr{C}(2)$ and also contains $\mathfrak{B}$, against Lemma 7.4. The proof is complete.

Lemma 7.8. Suppose that $\mathfrak{P}$ is of exponent 3 , order 81 and that $|\boldsymbol{Z}(\mathfrak{P})|=9$. Then $\boldsymbol{N}(\mathfrak{P})$ is the unique element of $\mathbb{C} \mathscr{S}(\mathbb{S})$ which contains $\mathfrak{P}$.

Proof. Suppose false. Let $\subseteq$ be a solvable subgroup of $\mathbb{B}$ which 
contains $\mathfrak{P}$ and is minimal subject to $\mathfrak{P} \not \mathfrak{S}$. Let $\mathfrak{P}_{0}=\boldsymbol{O}_{3}(\mathfrak{S})$. Since $Z(\mathfrak{P}) \subset \mathfrak{P}_{0} \subset \mathfrak{P}$, it follows that $\mathfrak{P}_{0}$ is abelian of order 27. Since $\mathfrak{S}$ is not 3-closed, it follows that $\mathfrak{S}=\mathfrak{P} \Omega$ where $\mathfrak{Q}$ is a quaternion group.

Let $\mathfrak{P}_{0}=\mathfrak{P}_{1} \times \mathfrak{P}_{2}$ where $\mathfrak{P}_{1}=C_{\mathfrak{P}_{0}}(\mathfrak{Q}), \mathfrak{P}_{2}=\left[\mathfrak{P}_{0}, \mathfrak{Q}\right]$. Thus, $\left|\mathfrak{P}_{i}\right|=$ $3^{i}, i=1,2$. Let $\tilde{\mathfrak{P}}_{1}=\mathfrak{P}_{2} \cap \boldsymbol{Z}(\mathfrak{P})$. Thus, $\boldsymbol{Z}(\mathfrak{P})=\mathfrak{P}_{1} \times \tilde{\mathfrak{P}}_{1}$ and $\mathfrak{P}^{\prime}=\tilde{\mathfrak{P}}_{1}$. Let $\mathfrak{Q}^{\prime}=\langle I\rangle \subseteq N(\mathfrak{B})$. Write $N(\mathfrak{B})=\mathfrak{B} \Re$ where $\mathfrak{R}$ is a complement to $\mathfrak{P}$ in $N(\mathfrak{P})$ which contains $I$. Since $\mathfrak{R}$ normalizes $\tilde{\mathfrak{P}}_{1}$, it follows that $\boldsymbol{A}_{\mathscr{S}}(\boldsymbol{Z}(\mathfrak{P}))$ is abelian. Hence, $\boldsymbol{Z}(\mathfrak{P}) \cap \boldsymbol{C}(I) \triangleleft \boldsymbol{N}(\mathfrak{P})$. Since $\boldsymbol{Z}(\mathfrak{P}) \cap \boldsymbol{C}(I)=$ $\mathfrak{P}_{1}$, we get that $N(\mathfrak{P}) \subseteq N\left(\mathfrak{P}_{1}\right)$. Since $I \in N(\mathfrak{P})$, it follows that $\mathfrak{P}_{1}$ may be characterized as the only subgroup of $\boldsymbol{Z}(\mathfrak{P})$ of order 3 which is normal in $N(\mathfrak{P})$ and is not contained in $\mathfrak{P}^{\prime}$.

Let $\Re$ be any solvable subgroup of $(S)$ which contains $\mathfrak{P}$. We will show that $\Re \subseteq N\left(\mathfrak{P}_{1}\right)$. We may assume that $\mathfrak{P} \nless \Re$. Let $\tilde{\mathfrak{P}}_{0}=O_{3}(\Re) \supset$ $\boldsymbol{Z}(\mathfrak{P})$. Thus, $\tilde{\mathfrak{R}}_{0}$ is abelian of order 27 . By our characterization of $\mathfrak{P}_{1}$, it follows that $\mathfrak{P}_{1} \triangleleft \Omega$, that is, $\Re \subseteq N\left(\Re_{1}\right)$.

Set $\mathfrak{M}=N\left(\mathfrak{P}_{1}\right)$, so that $\mathfrak{M}$ is the unique element of $\mathscr{C} \mathscr{S}(\mathbb{S})$ which contains $\mathfrak{P}$. Let $\mathfrak{U}$ be any elementary subgroup of $\mathfrak{P}$ of order 27 . Then $\mathfrak{P} \cong N(\mathfrak{U})$, so $N(\mathfrak{U}) \subseteq \mathfrak{M}$. Now let $A$ be any element of $\mathfrak{B}^{\sharp}$. We will show that $\boldsymbol{C}(A) \subseteq \mathfrak{M}$. This is clear if $A \in \boldsymbol{Z}(\mathfrak{P})$. Suppose $A \notin \boldsymbol{Z}(\mathfrak{P})$. Then $\boldsymbol{C}_{\mathfrak{B}}(A)=\mathfrak{U}$ is of order 27 and is abelian. Hence, $N(\mathfrak{U}) \subseteq \mathfrak{M}$. This implies that some $S_{3}$-subgroup of $C(A)$ is contained in $\mathfrak{M}$. If $C(A)$ contains a $S_{3}$-subgroup of 8 , then $C(A) \subseteq \mathfrak{M}$, by uniqueness of $\mathfrak{M}$. So suppose that $\mathfrak{U}$ is a $S_{3}$-subgroup of $C(A)$. Then since $И(\mathfrak{U})$ is trivial, we get that $\mathfrak{A} \triangleleft \boldsymbol{C}(A)$, so in any case, $\boldsymbol{C}(A) \subseteq \mathfrak{M}$.

Let $\mathfrak{F}$ be any non identity subgroup of $\mathfrak{B}$. We will show that

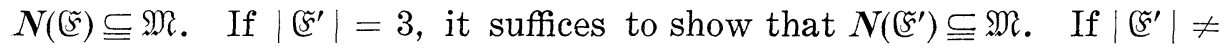
3 , then $\left[5\right.$ is abelian, since $\left|\mathfrak{P}^{\prime}\right|=3$. This, we may assume that $\mathbb{F}$ is abelian. By the preceding paragraph, $C(\mathfrak{S}) \subseteq \mathfrak{M}$. Let $\mathfrak{F}^{*}$ be a $S_{3}$ subgroup of $\boldsymbol{C}(\mathfrak{F})$. Then $N(\mathfrak{F})=C(\mathbb{F}) \cdot\left(N(\mathfrak{F}) \cap N\left(\mathfrak{F}^{*}\right)\right)$, so it suffices to

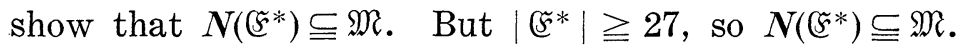

It is a consequence of the preceding results, that if $\mathfrak{F}$ is a solvable subgroup of $\mathbb{S}$ such that $\mathfrak{S} \cap \mathfrak{P}$ is noncyclic, then $\mathfrak{S} \subseteq \mathfrak{M}$.

Let $\mathfrak{B}=\mathfrak{S} \cap N\left(\mathfrak{Q}^{\prime}\right)$ so that $\mathfrak{B}$ is noncyclic. Hence, $N\left(\mathfrak{\Omega}^{\prime}\right) \subseteq \mathfrak{M}$. This is not the case since $N\left(\mathfrak{Q}^{\prime}\right)$ contains an element of $\mathscr{C}(2)$, while $\mathfrak{M}$ contains an element of $\mathscr{C}(3)$.

\section{A characterization of $E_{2}(3)$.}

THEOREM 8.1. $E_{2}(3)$ is the only simple group (s) with the following properties:

(i) 1 is the only 3-signalizer of (S).

(ii) The center of a $S_{3}$-subgroup of $\$$ is is noncyclic.

(iii) The normalizer of every nonidenty 3-subgroup of (B) is solvable. 
(iv) The centralizer of every involution of (S) is solvable.

(v) $S_{2}$-subgroup of $\&$ contain normal elementary subgroups of order 8.

(vi) If $\mathfrak{I}$ is a $S_{2}$-subgroup of $\mathbb{S}$ and $\mathfrak{A} \in \mathscr{S}_{\text {cno }}(\mathfrak{I})$, then $И(\mathfrak{A})$ is trivial.

(vii) $\quad 2 \sim 3$.

The proof of Theorem 8.1 is elaborate. I am indebted to J. Tits for helpful discussion.

We first derive some properties of $E_{2}(q)$. We use the notation and calculations of Ree [30]. In addition, we let $\mathfrak{B}=\mathfrak{H} \mathfrak{S}, \mathfrak{R}=\left\langle\mathfrak{S}, \omega_{a}, \omega_{b}\right\rangle$. $F_{q}$ is the field of $q=p^{n}$ elements, and if $x \in F_{q}$, then $\operatorname{tr}(x)=\operatorname{tr}_{F_{q} / F_{p}}(x)=$ $\Sigma x^{\sigma}, \sigma$ ranging over all the automorphisms of $F_{q}$. If $r \in \Sigma$, then $\mathfrak{X}_{r}=$ $\left\langle x_{r}(t) \mid t \in F_{q}\right\rangle$.

We need the usual sort of omnibus lemma.

Lemma 8.1. Let $\mathfrak{U}, \mathfrak{B}, \mathfrak{S}, \mathfrak{R}$ denote the subgroups of $E_{2}(q)$ given above.

(i ) $\mathfrak{W}_{0}=\left\langle\omega_{b}^{2} \omega_{a}, \omega_{a}^{2} \omega_{b}\right\rangle$ is a dihedral group of order 12 and is a complement to $\mathfrak{S}$ in $\mathfrak{R}$.

(ii) $\mathfrak{S}$ is the direct product of two cyclic groups of order $q-1$, with generators $H_{1}=h\left(\chi_{a, z}\right), H_{2}=h\left(\chi_{b, z}\right)$. Here $z$ is a generator for $F_{q}^{*}$. If $W_{1}=\omega_{b}^{2} \omega_{a}, W_{2}=\omega_{a}^{2} \omega_{b}$, then

$$
\begin{array}{ll}
W_{1}^{-1} H_{1} W_{1}=H_{1}^{-1}, & W_{1}^{-1} H_{2} W_{1}=H_{1} H_{2}, \\
W_{2}^{-2} H_{1} W_{2}=H_{1} H_{2}^{3}, & W_{2}^{-1} H_{2} W_{2}=H_{2}^{-1},
\end{array}
$$

(iii) If $q$ is a power of 3 and $\nu$ is a nonsquare in $F_{q}$, then

$$
\left\{x_{3 a+2 b}(1), x_{2 a+b}(1), x_{3 a+2 b}(1) x_{2 a+b}(1), x_{a+b}(1) x_{3 a+b}(1), x_{a+b}(1) x_{3 a+b}(\nu)\right\}
$$

is a set of representatives for the conjugacy classes of $E_{2}(q)$ of order 3. If $c \in F_{q}$ satisfies $\operatorname{tr}(c)=1$, then $\left\{x_{a}(1) x_{b}(1) x_{3 a+b}(e c), e=0,1,-1\right\}$ is a set of representatives for the conjugacy classes of elements of $E_{2}(q)$ of order 9 . $\mathfrak{U}$ is of exponent 9 .

(iv) Assume that $q$ is odd.

(a) Let $\widetilde{\mathfrak{B}}=C_{\mathfrak{B}}\left(\omega_{a}^{2}\right), \tilde{\mathfrak{R}}=C_{\mathfrak{R}}\left(\omega_{a}^{2}\right)$. Let $\mathbb{E}=C_{E_{2}(q)}\left(\omega_{a}^{2}\right)$. Then

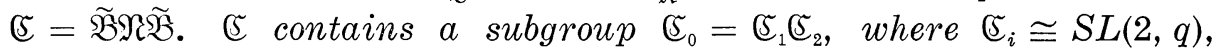
$i=1,2, \mathfrak{\Im}_{1} \cap \mathfrak{S}_{2}=\boldsymbol{Z}\left(\mathfrak{S}_{i}\right)=\left\langle\omega_{a}^{2}\right\rangle$, $\mathfrak{S}_{1}$ and $\mathfrak{\Im}_{2}$ commute elementwise and $\left|\mathfrak{S}: \mathfrak{S}_{0}\right|=2$. Furthermore, $\mathfrak{S}_{i} \triangleleft \mathfrak{S}, i=1,2$.

(b) For $i=1,2$, let $\alpha_{i}$ be the isomorphism from $\mathfrak{E}_{i}$ to $S L(2, q)$ induced by $x_{r_{i}}(t) \rightarrow\left(\begin{array}{ll}1 & t \\ 0 & 1\end{array}\right), z_{-r_{i}}(t) \rightarrow\left(\begin{array}{ll}1 & 0 \\ t & 1\end{array}\right)$, where $r_{1}=a, r_{2}=$ $3 a+2 b$. Each element $X$ in $\mathfrak{S}-\mathfrak{E}_{0}$ induces an automorphism $\phi_{X}^{(i)}$ of $\mathfrak{E}_{i}$ such that $\alpha_{i} \varphi_{X}^{(i)} \alpha_{i}^{-1}$ coincides with the automorphism of $S L(2, q)$ 
induced by an element of $G L(2, q)$ whose determinant is a nonsquare.

( c) There are involutions in $\sqrt{5}-\mathfrak{F}_{0}$. If $X$ is an involution in $\sqrt{ } \sqrt{ }-\mathfrak{V}_{0}$, and $q \equiv \varepsilon(\bmod 4), \varepsilon= \pm 1$, then $C(X) \cap \mathfrak{S}_{0}$ has order $2(q+\varepsilon)^{2}$ and

$$
\begin{aligned}
C(X) \cap \mathfrak{\subseteq}_{0} & \cong g p\langle w, x, y, z| x^{(q+\varepsilon) / 2} \\
& =y^{(q+\varepsilon) / 2}=w, w^{2}=1, x y=y x, z^{-1} x z \\
& \left.=x^{-1}, z^{-1} y z=y^{-1}, z^{2}=1\right\rangle .
\end{aligned}
$$

( v) If $q$ is odd, then $i\left(E_{2}(q)\right)=1$.

Proof. The Weyl group of $G_{2}$ is dihedral of order 12 , so $w_{a} w_{b}$ is of order 6. By (1.8) of [30], $\left(\omega_{a} \omega_{b}\right)^{6}=h(\chi)$, for some $\chi \in X$. We show that $\chi=1$. It suffices to show that $\chi(a)=\chi(b)=1$, that is, $\eta_{a}=$ $\eta_{b}=1$. This follows readily from table (3.4) of [30]. Since $\omega_{a}^{-1} \omega_{b}^{2} \omega_{a}=$ $\omega_{b}^{2} \omega_{a}^{2}$, and $\omega_{b}^{-1} \omega_{a}^{2} \omega_{b}=\omega_{a}^{2} \omega_{b}^{2}$, the elements $\omega_{b}^{2} \omega_{a}$ and $\omega_{a}^{2} \omega_{b}$ are involutions. We have $\left(\omega_{b}^{2} \omega_{a}\right)\left(\omega_{a}^{2} \omega_{b}\right)=\omega_{b}^{2} \omega_{a}^{-1} \omega_{b} \sim \omega_{b}^{-1} \omega_{a}^{-1}=\left(\omega_{a} \omega_{b}\right)^{-1}$, proving (i).

It is convenient for calculations to use the following character table:

\begin{tabular}{l|l|l} 
& $a$ & $b$ \\
\hline$\chi_{a, z}$ & $z^{2}$ & $z^{-3}$ \\
$\chi_{b, z}$ & $z^{-1}$ & $z^{2}$
\end{tabular}.

To determine this character table, we need to compute the values $u(r), u, r \in \Sigma$ (see [30], p. 433). The relevant values of $u(r)$ are given as follows:

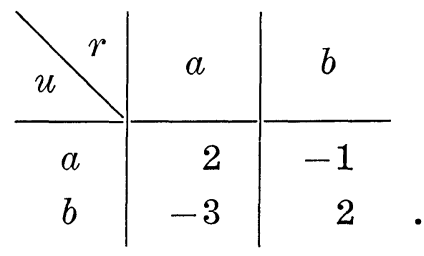

Using this table, we compute the values $w_{r}(s)$, as follows:

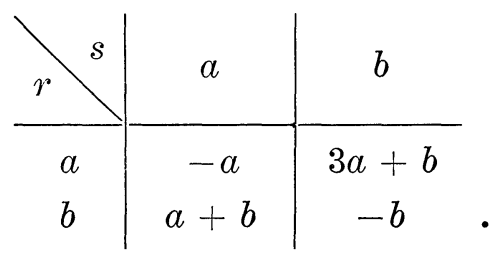

(Using the geometric interpretation of $w_{r}$, we can read these results directly from Figure 1 of [30].)

We next compute $w_{r}(\chi)$ for $r=a, b$ and $\chi=\chi_{a, z}, \chi_{b, z}$. For example, 
$\left[w_{a}\left(\chi_{a, z}\right)\right](a)=\chi_{a, z}\left(w_{a}(a)\right)=\chi_{a, z}(-a)=\chi_{a, z}(a)^{-1}=z^{-2}$. Continuing in this fashion, we get the following table of values:

\begin{tabular}{l|l|l} 
& \multicolumn{1}{|c|}{$a$} & \multicolumn{1}{|c}{$b$} \\
\hline$w_{a}\left(\chi_{a, z}\right)$ & $z^{-2}$ & $z^{3}$ \\
$w_{a}\left(\chi_{b, z}\right)$ & $z$ & $z^{-1}$ \\
$w_{b}\left(\chi_{a, z}\right)$ & $z^{-1}$ & $z^{3}$ \\
$w_{b}\left(\chi_{b, z}\right)$ & $z$ & $z^{-2}$
\end{tabular}.

Referring back to the character table, we have

$$
\begin{aligned}
& w_{a}\left(\chi_{a, z}\right)=\chi_{a, z}^{-1}, w_{a}\left(\chi_{b, z}\right)=\chi_{a, z} \chi_{b, z}, \\
& w_{b}\left(\chi_{a, z}\right)=\chi_{a, z} \chi_{b, z}^{3}, w_{b}\left(\chi_{b, z}\right)=\chi_{b, z}^{-1} .
\end{aligned}
$$

The map from $X$ to $\mathfrak{S}$ induced by $\chi_{r, z} \rightarrow h\left(\chi_{r, z}\right)$ is an isomorphism, since $X$ and $\mathfrak{S}$ have order $(q-1)^{2}$. The previous information, together with (1.7) of [30] implies that (ii) holds.

Let $\mathfrak{U}_{1}=\mathfrak{U} \cap \mathfrak{U}^{\omega_{a}}, \mathfrak{U}_{2}=\mathfrak{U} \cap \mathfrak{U}^{\omega_{b}}$. By using (3.10) of [30] it is straightforward to verify that $\mathfrak{u}_{1} \cup \mathfrak{u}_{2}$ is the set of elements of $\mathfrak{u}$ of order 1 or 3 . This then implies easily that every element of $E_{2}(q)$ of order 3 is conjugate to an element of $\mathfrak{U}_{1} \cap \mathfrak{U}_{2}=\left\langle\mathfrak{X}_{a+b}, \mathfrak{X}_{2 a+b}, \mathfrak{X}_{3 a+b}, \mathfrak{X}_{3 a+2 b}\right\rangle$. Since $\mathfrak{B}=N(\mathfrak{U})$, it follows from Lemma 14.3 .1 of [21] that elements of $Z(\mathfrak{U})$ are conjugate in $E_{2}(q)$ only if they are conjugate in $\mathfrak{B}$. Since the action of $\mathfrak{K}$ on $\boldsymbol{Z}(\mathfrak{U})=\left\langle\mathfrak{X}_{2 a+b}, \mathfrak{X}_{3 a+2 b}\right\rangle$ is determined by (1.5) of [30] and our character table, it follows that any element of $E_{2}(q)^{\#}$ which is conjugate to an element of $\boldsymbol{Z}(\mathfrak{U})$ is conjugate to exactly one of $x_{2 a+b}(1)$, $x_{3 a+2 b}(1), x_{2 a+b}(1) x_{3 a+2 b}(1)$. Furthermore, since the Weyl group permutes transitively the roots of a given length, and since $2 a+b$ and $3 a+2 b$ have different lengths, it follows that every element of the shape $x_{r}(t), r \in \Sigma$, is conjugate to an element of $\boldsymbol{Z}(\mathfrak{U})$. Suppose $x \in \mathfrak{H}_{1} \cap \mathfrak{U}_{2}$, $x=x_{a+b}\left(t_{1}\right) x_{2 a+b}\left(t_{2}\right) x_{3 a+b}\left(t_{3}\right) x_{3 a+2 b}\left(t_{4}\right)$, and that $x$ is conjugate to no element of $Z(\mathfrak{U})$. Hence, either $t_{1} \neq 0$ or $t_{3} \neq 0$. Suppose $t_{3}=0$. Conjugation by $x_{a}\left(-t_{1}^{-1} t_{2} / 2\right)$ enables us to assume that $t_{2}=0$. Conjugation by $\omega_{a}$ then yields that $x$ is conjugate to an element of $Z(\mathfrak{H})$. Hence, $t_{3} \neq 0$. Suppose $t_{1}=0$. Conjugation by $x_{b}\left(t_{3}^{-1} t_{4}\right)$ enables us to assume that $t_{4}=0$. Conjugation by $\omega_{b}$ yields that $x$ is conjugate to an element of $\boldsymbol{Z}(\mathfrak{U})$. Hence, $t_{1} t_{3} \neq 0$. Conjugation by $x_{a}\left(-t_{1}^{-1} t_{2} / 2\right) x_{b}\left(t_{3}^{-1} t_{4}\right)$ enables us to assume that $t_{2}=t_{4}=0$. Since $h\left(\chi_{a, z}\right) x_{a+b}\left(t_{1}\right) h\left(\chi_{a, z}\right)^{-1}=x_{a+b}\left(z^{-1} t_{1}\right)$, we may assume that $t_{1}=1$. Since $h\left(\chi_{a, z} \chi_{b, z}\right)$ centralizes $x_{a+b}(1)$ and since $h\left(\chi_{a, z} \chi_{b, z}\right) x_{3 a+b}\left(t_{3}\right) h\left(\chi_{a, z} \chi_{b, z}\right)^{-1}=x_{3 a+b}\left(t_{3} z^{2}\right)$, we may assume that $t_{3}=$ 1 or $\nu$. A direct calculation shows that the centralizer of $x_{a+b}(1) x_{3 a+b}(u)$ does not contain a $S_{3}$-subgroup of $E_{2}(q)$ for any $u$ in $F_{q}^{*}$, and a further calculation shows that $x_{a+b}(1) x_{3 a+b}(1)$ is not conjugate to $x_{a+b}(1) x_{3 a+b}(\nu)$, 
completing the proof of the first part of (iii).

If $t u \neq 0$, it is easy to verify that $x_{a}(t) x_{b}(u) x$ has order 9 for all $x$ in $\mathfrak{U}_{1} \cap \mathfrak{U}_{2}$ and that $\left(x_{a}(t) x_{b}(u)\right)^{3}=\left(x_{a}(t) x_{b}(u) x\right)^{3}$. A calculation shows that $\mathfrak{F}$ permutes transitively the elements $x_{a}(t) x_{b}(u), t u \in F_{q}^{*}$, so every element of $E_{2}(q)$ of order 9 is conjugate to an element of the shape $x_{a}(1) x_{b}(1) x$, with $x$ in $\mathfrak{U}_{1} \cap \mathfrak{U}_{2}$. Let $x=x_{a+b}\left(t_{1}\right) x_{2 a+b}\left(t_{2}\right) x_{3 a+b}\left(t_{3}\right) x_{3 a+2 b}\left(t_{4}\right)$. Conjugation by $x_{a}(u)$ enables us to assume that $t_{3}=0$. A further conjugation by $x_{a+b}\left(u_{1}\right) x_{3 a+b}\left(u_{2}\right)$ enables us to assume that $t_{2}=t_{4}=0$. Thus, it suffices to show that

$$
x_{a}(1) x_{b}(1) x_{a+b}(u) \text { is conjugate to } x_{a}(1) x_{b}(1) x_{a+b}(v)
$$

if and only if $\operatorname{tr}(u)=\operatorname{tr}(v)$. If $g$ conjugates the first element into the second then $g$ centralizes $\left(x_{a}(1) x_{b}(1)\right)^{3}$. A calculation shows that the centralizer of $\left(x_{a}(1) x_{b}(1)\right)^{3}$ is $\mathfrak{u}$, and a further calculation completes the proof of (iii).

By a direct calculation, $\widetilde{\mathfrak{B}}=\left\langle\mathfrak{X}_{a}, \mathfrak{X}_{3 a+2 b}, \mathfrak{S}\right\rangle, \widetilde{\mathfrak{R}}=\left\langle\mathfrak{S}, \omega_{a},\left(\omega_{a} \omega_{b}\right)^{3}\right\rangle$. Suppose $\omega_{a}^{2}$ centralizes $x h \omega x^{\prime}, x \in \mathfrak{H}, h \in \mathfrak{S}, \omega \in \mathfrak{W}_{0}, x^{\prime} \in \mathfrak{U}_{w}, w$ being the image of $\omega$ in the Weyl group. Then the normal form implies that $x, x^{\prime}, h, \omega \in C\left(\omega_{a}^{2}\right)$, so the first assertion of (iv) is proved.

Let $\mathfrak{F}_{1}=\left\langle\mathfrak{X}_{a}, \mathfrak{X}_{-a}\right\rangle, \mathfrak{F}_{2}=\left\langle\mathfrak{X}_{3 a+2 b}, \mathfrak{X}_{-(3 a+2 b)}\right\rangle$, so that $\mathfrak{F}_{1} \cong \mathfrak{F}_{2} \cong S L(2, q)$. Clearly, $\mathfrak{E}_{1}$ and $\mathfrak{F}_{2}$ commute elementwise. Since $\chi_{3 a+2 b,-1}=\chi_{a,-1}$, it follows that $\mathfrak{夭}_{1} \cap \mathfrak{E}_{2}=\left\langle\omega_{a}^{2}\right\rangle$, so that $\mathfrak{E}_{0}=\mathfrak{C}_{1} \mathfrak{E}_{2}$ is the central product of $\mathfrak{S}_{1}$ and $\mathfrak{S}_{2}$. Setting $\widetilde{\mathfrak{U}}=\mathfrak{U} \cap \widetilde{\mathfrak{B}}$, we have

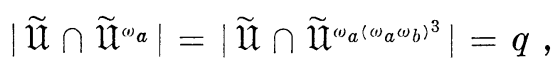

and $\tilde{\mathfrak{U}} \cap \widetilde{\mathfrak{U}}^{\left(\omega_{1} a^{(\omega)}\right)^{3}}=1$, it follows that $|\mathfrak{S}|=q^{2}(q-1)^{2}\left(1+2 q+q^{2}\right)$. Hence, $(*)$

$$
\left|\mathfrak{E}: \mathfrak{S}_{0}\right|=\left|\mathfrak{S}_{\mathfrak{C}}: \mathfrak{S} \cap \mathfrak{S}_{0}\right|=2 \text {. }
$$

Since $\mathfrak{S}_{\mathfrak{C}}$ normalizes $\mathfrak{X}_{r}$ for all $r$ in $\Sigma$, (iv) (a) is proved.

We observe that by (1.5) of [30],

$$
\begin{aligned}
h\left(\chi_{b, z}\right) x_{a}(t) h\left(\chi_{b, z}\right)^{-1} & =x_{a}\left(z^{-1} t\right), \\
h\left(\chi_{b, z}\right) x_{-a}(t) h\left(\chi_{b, z}\right)^{-1} & =x_{-a}(z t) .
\end{aligned}
$$

Hence, if $\eta=\phi_{h\left(\chi_{b, z}\right)}^{(1)}$ denotes the automorphism of $\mathfrak{F}_{1}$ induced by $h\left(\chi_{b, z}\right)^{-1}$, then $\alpha_{1} \eta \alpha_{1}^{-1}$ is the automorphism of $S L(2, q)$ induced by the map

$$
\left(\begin{array}{ll}
1 & t \\
0 & 1
\end{array}\right) \rightarrow\left(\begin{array}{cc}
1 & z^{-1} t \\
0 & 1
\end{array}\right), \quad\left(\begin{array}{cc}
1 & 0 \\
t & 1
\end{array}\right) \rightarrow\left(\begin{array}{cc}
1 & 0 \\
z t & 1
\end{array}\right)
$$

This automorphism therefore coincides with the automorphism induced by $\left(\begin{array}{ll}z & 0 \\ 0 & 1\end{array}\right)$. A similar argument applies to $\mathfrak{F}_{2}$. Since $\mathbb{C}-\mathbb{E}_{0}$ coincides with the coset $\mathfrak{S}_{0} h\left(\chi_{b, z}\right)$ whenever $z$ is not a square of $F_{q}^{*}$, the proof of $(\mathrm{iv})(\mathrm{b})$ is complete. 
Let $K=\left(W_{1} W_{2}\right)^{3}$. By (i), $K$ is an involution, and by (ii), $K$ inverts $\mathfrak{S}$. Thus, $\mathfrak{S} K$ is a set of involutions in $\mathfrak{C}$. If $\mathfrak{S}_{\mathfrak{L}} K \subseteq \mathfrak{S}_{0}$, we get $\mathfrak{S} \subseteq\left\langle\mathfrak{S}_{\mathcal{C}} K\right\rangle \subseteq C_{0}$, against $\left(^{*}\right)$. So $\mathfrak{C}-\mathbb{F}_{0}$ contains an involution.

We will use (iv)(b) in the proof of (iv)(c). First, suppose $\varepsilon=-1$. In this case, -1 is not a square in $F_{q}$. Since $\widetilde{r}_{1}$ and $\widetilde{r}_{2}$ commute elementwise, we assume without loss of generality that for $i=1,2$, $\alpha_{i} \varphi_{X}^{(i)} \alpha_{i}^{-1}$ is the automorphism of $S L(2, q)$ induced by $\left(\begin{array}{rr}-1 & 0 \\ 0 & 1\end{array}\right)$. Hence, $\mathfrak{夭}_{i} \cap C(X)$ is cyclic of order $q-1$. Since the commutator of $\left(\begin{array}{rr}-1 & 0 \\ 0 & 1\end{array}\right)$ and $\left(\begin{array}{rr}0 & 1 \\ -1 & 0\end{array}\right)$ is $-I$, and since $\left(\begin{array}{rr}0 & 1 \\ -1 & 0\end{array}\right)$ inverts $\left(\begin{array}{ll}x & 0 \\ 0 & x^{-1}\end{array}\right)$ for all $x \in F_{q}^{*}$, (iv)(c) follows in this case.

Now suppose $\varepsilon=1$. In this case, -1 is a square in $F_{q}$. Choose $a, b \in F_{q}$ such that $a^{2}+b^{2}=c$ is a nonsquare. We may assume that for $i=1,2, \alpha_{i} \varphi_{X}^{(i)} \alpha_{i}^{-1}$ is the automorphism of $S L(2, q)$ induced by $\left(\begin{array}{rr}a & b \\ b & -a\end{array}\right)$. A short calculation shows that (iv)(c) holds.

Since $\left|\boldsymbol{C}\left(\omega_{a}^{2}\right)\right|=\left(q\left(q^{2}-1\right)\right)^{2}$, it follows that $\boldsymbol{C}\left(\omega_{a}^{2}\right)$ contains a $S_{2}$ subgroup of $E_{2}(q)$. Thus, to prove (v), it suffices to show that each involution $X$ of $\widetilde{F}$ is conjugate to $\omega_{a}^{2}$ in $E_{2}(q)$. Since $E_{2}(q)$ is simple, Lemma 5.38 (a)(i) implies that $X$ is conjugate in $E_{2}(q)$ to an element of $\mathbb{E}_{0}$.

Thus, it suffices to show that all involutions of $\mathfrak{F}_{0}$ are conjugate in $E_{2}(q)$. Since $\mathbb{E}_{i}$ has just involution for $i=1,2$, it follows that every involution of $\widetilde{\Xi}_{0}$ different from $\omega_{a}^{2}$ is of the shape $I_{1} I_{2}$ where $I_{i} \in \mathfrak{S}_{i}$ and $I_{i}^{2}=\omega_{a}^{2}$. Since $\mathfrak{S}_{i}$ has just 1 conjugacy class of elements of order 4 , it follows that $\mathfrak{\Im}_{0}$ has two conjugacy classes of involutions.

Case 1. Every involution of $\mathfrak{S}$ is in $\mathfrak{S}_{0}$.

By (ii), all involutions of $\mathfrak{S}$ are fused in $\mathfrak{R}$. By the preceding paragraph, (v) follows.

Case 2. $J$ is an involution of $\left(\mathfrak{C}-\mathbb{S}_{0}\right) \cap \mathfrak{F}$.

Set $I=\omega_{a}^{2}, K=\left(W_{1} W_{2}\right)^{3}$, so that $K$ inverts $\mathscr{S}$ and so centralizes $I$ and $J$. Let $\mathfrak{A}=\langle I, J, K\rangle$. By (ii), the involutions of $\mathfrak{U}$ are fused in $\mathfrak{R}$ as follows:

$$
I \sim J \sim I J, I K \sim J K \sim I J K .
$$

It is clear that in $\sqrt{5}$ all the involutions of $\sqrt{5}-\mathfrak{C}_{0}$ are conjugate. Let $\mathfrak{V}_{0}=\mathfrak{U} \cap \mathfrak{S}_{0}$. Thus, $\mathfrak{V}_{0}$ is one of $\langle I, K\rangle,\langle I, J K\rangle$. Suppose $\mathfrak{U}_{0}=$ $\langle I, K\rangle$. Then, $J, J I, J K, J I K$ are the involutions of $\mathfrak{A}-\mathfrak{A}_{0}$, so are all conjugate in $\mathfrak{S}_{\text {. }}$. Since $K \in \mathfrak{S}_{0}, K$ and $K I$ are conjugate in $\mathfrak{S}_{0}$. Thus, all involutions of $\mathfrak{A}$ are conjugate in $E_{2}(q)$, so (v) follows. Suppose $\mathfrak{A}_{0}=\langle I, J K\rangle$. Then, $J, J I, K, K I$ are the involutions of $\mathfrak{A}-\mathfrak{A}_{0}$, so are all conjugate in $\mathfrak{5}$. Again all involutions of $\mathfrak{A}$ are conjugate in $E_{2}(q)$. The proof of (v) is complete. 
LEMMA 8.2. (a) Suppose $B$ is a finite group and $\mathfrak{B}$ is a foursubgroup of 88. Suppose also that whenever $I$ and $J$ are distinct involutions of $\mathfrak{B}, I$ and $I J$ are conjugate in $C(J)$. Then $\boldsymbol{A}(\mathfrak{B})=$ Aut $(\mathfrak{B})$.

(b) $\quad \boldsymbol{A}_{E_{2}(3)}(\mathfrak{B})=\operatorname{Aut}(\mathfrak{B})$ for every four-subgroup $\mathfrak{B}$ of $E_{2}(3)$.

Proof. Choose $X$ in $C(J)$ such that $X^{-1} I X=I J$. Thus,

$$
X \in N(V) \cap C(J) \text {. }
$$

Replacing the pair $I, J$ by the pair $J, I$ we choose $Y$ in $C(I)$ with $Y^{-1} J Y=I J$. Then $\mathfrak{U}=\langle X Y\rangle$ permutes $I, J, I J$ cyclically. Thus, $\langle X, Y\rangle$ maps onto Aut (iB), proving (a).

Let $\mathfrak{B}$ be a four-subgroup of $E_{2}(3)$ and let $I, J$ be distinct involutions of $\mathfrak{B}$. We will produce $X$ in $C(J)$ such that $X^{-1} I X=I J$. We may assume that $J=\omega_{a}^{2}$, since $i\left(E_{2}(3)\right)=1$. Since $\boldsymbol{O}_{2}\left(C\left(\omega_{a}^{2}\right)\right)$ is extra special, we are done in case $I \in \boldsymbol{O}_{2}\left(\boldsymbol{C}\left(\omega_{a}^{2}\right)\right)$. If $I \notin \boldsymbol{O}_{2}\left(\boldsymbol{C}\left(\omega_{a}^{2}\right)\right)$, then $I$ induces an outer automorphism of both quaternion subgroups of $\boldsymbol{O}_{2}\left(\boldsymbol{C}\left(\omega_{a}^{2}\right)\right)$, so again $X$ is available. Now (b) follows from (a).

We omit the proof that $\mathfrak{} \subseteq=C_{E_{2}(3)}\left(\omega_{a}^{2}\right)$ has exactly $19+72$ involutions; namely, $\boldsymbol{O}_{2}(\mathbb{E})$ has exactly 19 involutions, while all involutions of $\sqrt{5}-\boldsymbol{O}_{2}(\sqrt{ })$ are conjugate in $\sqrt{5}$. Furthermore, it is straightforward to verify that 5 has exactly 3 conjugacy classes of elementary subgroups of order 8. Representatives $\mathfrak{F}_{1}, \mathfrak{F}_{2}, \mathfrak{F}_{3}$ for these classes may be chosen so that if $\mathfrak{I}$ denotes a fixed $S_{2}$-subgroup of $\mathfrak{E}$, then $\mathfrak{F}_{i} \triangleleft \mathfrak{I}$, and $\mathbb{F}_{i} \subseteq O_{2}(\mathbb{C}), i=1,2$.

We argue that $\mathfrak{F}_{1}$ and $\mathscr{F}_{2}$ are not conjugate in $E_{2}(3)$. Suppose $\mathfrak{F}_{1}^{G}=\mathfrak{F}_{2}$. Then $\mathfrak{I}^{G}$ normalizes $\mathfrak{F}_{2}$, as does $\mathfrak{T}$. Then $\mathfrak{T}^{G}=\mathfrak{I}^{N}$ for some $N$ in $N\left(\mathfrak{F}_{2}\right)$. Hence, $G N^{-1} \in N(\mathfrak{I})=\mathfrak{I}$, so $G \in \mathfrak{I} N \subseteq N\left(\mathfrak{F}_{2}\right)$. Since $\mathfrak{F}_{1}^{G}=$ $\mathfrak{F}_{2}$, we get $\mathfrak{F}_{1}=\mathfrak{F}_{2}$, a contradiction.

Set $\mathfrak{B}=\mathfrak{F}_{1} \cap \mathfrak{F}_{2}$ so that $\mathfrak{B}$ is a four-subgroup of $\mathfrak{I}$ and $\boldsymbol{O}_{2}(\mathfrak{I}) \cap C(\mathfrak{B})=$ $\mathfrak{F}_{1} \mathfrak{F}_{2}$ is the direct product of a group of order 2 and a dihehral group of order 8. Let $\mathfrak{D}=\boldsymbol{C}_{E_{2}(3)}(\mathfrak{B})=\boldsymbol{C}_{\mathfrak{T}}(\mathfrak{B})$, a group of order 32 . We omit the proof that $\mathfrak{D}$ has exactly 4 elementary subgroups of order 8 , among which are $\mathfrak{F}_{1}$ and $\mathfrak{F}_{2}$. By Lemma $8.2(\mathrm{~b}), N(\mathfrak{B})$ has an element $A$ of order 3 which permutes transitively the involutions of $\mathfrak{B}$. If $A$ normalizes both $\xi_{1}$ and $\mathfrak{F}_{2}$, then $A$ normalizes the derived group of $\mathfrak{F}_{1} \mathfrak{\xi}_{2}$, that is, $A$ normalizes $\left\langle\omega_{a}^{2}\right\rangle$. Since this is not the case, we can choose $i$ in $\{1,2\}$ so that the orbit of $\mathfrak{F}_{i}$ under $\langle A\rangle$ has 3 elements. Since $\mathfrak{F}_{1}$ and $\mathfrak{F}_{2}$ are in different orbits under $\langle A\rangle$, it follows that $A$ normalizes $\mathfrak{F}_{j}$, where $\{i, j\}=\{1,2\}$.

We omit the proof that $N\left(\Im_{j}\right) \cap \sqrt{ }$ permutes transitively the involutions of $\xi_{j}-\left\langle\omega_{a}^{2}\right\rangle$. Since $A$ does not centralize $\omega_{a}^{2}$, it follows that $N\left(\mathfrak{F}_{j}\right)$ permutes transitively the involutions of $\mathfrak{F}_{j}$. Thus, $\left|N\left(\mathfrak{F}_{j}\right)\right|=$ $7 \cdot\left|N\left(\mathfrak{\mho}_{j}\right) \cap \mathfrak{S}\right|=8 \cdot 24 \cdot 8$. Hence, 


$$
\mathfrak{A}_{E_{2}(3)}\left(\mathfrak{F}_{j}\right)=\operatorname{Aut}\left(\mathfrak{F}_{j}\right) \text {. }
$$

We have proved (a) of the next lemma.

Lemma 8.3. (a) $E_{2}(3)$ is not an $N$-group.

(b) $E_{2}(3)$ satisfies the hypotheses of Theorem 8.1.

Proof. It suffices to verify (b).

By Lemma 8.1 (iv), hypothesis (iv) of Theorem 8.1 is satisfied. By definition of $\sim$, so is hypothesis (vii), $C_{E_{2}(3)}\left(\omega_{a}^{2}\right)$ being the relevant solvable group. Hypothesis (ii) is clearly satisfied, since

$$
\boldsymbol{Z}(\mathfrak{U})=\left\langle\mathfrak{X}_{2 a+b}, \mathfrak{X}_{3 a+2 b}\right\rangle .
$$

Clearly, 1 is the only 2-signalizer of $C\left(\omega_{a}^{2}\right)$, so if $\mathfrak{I}$ is a $S_{2}$-subgroup of $C\left(\omega_{a}^{2}\right)$ and $\mathbb{Q}$ is a nonidentity $2^{\prime}$-subgroup of $E_{2}(3)$ normalized by $\mathfrak{I}$, then $\omega_{a}^{2}$ inverts $\mathbb{R}$, so $\&$ is abelian. Furthermore, $\mathbb{Z}$ is a 3 -group, as every $\{2,3\}^{\prime}$-subgroup of $E_{2}(3)$ is cyclic. Since $\mathbb{Z}$ is a faithful $\mathfrak{I}$-module, $|\mathfrak{Q}| \geqq 3^{4}$. Since $\mathfrak{U}$ has no abelian subgroup of order $3^{5}$, it follows that is elementary of order $3^{4}$. It is straightforward to verify that every elementary subgroup of $\mathfrak{u}$ of order $3^{4}$ is conjugate to

$$
\left\langle\mathfrak{X}_{2 a+b}, \mathfrak{X}_{3 a+b}, \mathfrak{X}_{a+b}, \mathfrak{X}_{3 a+2 b}\right\rangle ;
$$

the normalizer of this last group is $\mathfrak{B}$, so does not contain a $S_{2}$-subgroup of $E_{2}(3)$. Thus, 1 is the only 2-signalizer of $E_{2}(3)$. It is trivial to verify that 1 is the only 3 -signalizer of $E_{2}(3)$, so hypothesis (i) is verified.

Since $E_{2}(3)$ is of order $2^{6} \cdot 3^{6} .7 .13$, and since the centralizer of every nonidentity 3 -element of $E_{2}(3)$ is a 2,3 -group, it is easy to check that hypothesis (iii) is satisfied. Since $S_{2}$-subgroups of $E_{2}(3)$ are of order 64 , and since $\left(^{* *}\right)$ holds, hypothesis (v) is satisfied.

Suppose that $\mathfrak{A} \in \mathscr{S} \mathscr{C} \mathscr{N}_{3}(\mathfrak{P})$ for a $S_{2}$-subgroup $\mathfrak{P}$ of $E_{2}(3)$, and $\mathfrak{B}$ is minimal nontrivial element of $\boldsymbol{U}(\mathfrak{H})$. Then $\mathfrak{A} \mathfrak{B}$ is contained in the centralizer of an involution; say $\mathfrak{A} \mathfrak{B} \leqq \mathbb{}=C\left(\omega_{a}^{2}\right)$. But, by Lemma 8.1 (iv), 5 contains no nontrivial 2 '-subgroup $\mathfrak{B}$ for which $N_{\mathfrak{E}}(\mathfrak{B})$ contains an elementary subgroup of order $2^{3}$. This contradiction proves that $И(\mathfrak{A})=\{1\}$, which is hypothesis (vi). The proof is complete.

The remaining results in this section are proved under the hypothesis that (8) satisfies the hypothesis of Theorem 8.1.

LEMma 8.4. (i) (B) satisfies Hypothesis 7.4.

(ii) (S) satisfies Hypothesis 7.1 for $p=2$ and for $p=3$.

Proof. We first show that $\mathscr{S C C S}_{3}(3) \neq \varnothing$. Suppose false. Let $\mathfrak{P}$ be a $S_{3}$-subgroup of $\mathbb{S}$. Since $\mathscr{S} \mathscr{C} \mathscr{N}_{3}(\mathfrak{P})=\varnothing$, it follows that 
$\Omega_{1}(\mathfrak{P})=\Omega_{1}(\boldsymbol{Z}(\mathfrak{P}))$ is of type $(3,3)$. This implies that every 3 -solvable subgroup of $\mathbb{B S}$ has 3 -length at most 1 . Since 1 is the only 3 -signalizer of $\mathfrak{B S}$, it follows that $\mathfrak{P}=\boldsymbol{C}\left(\Omega_{1}(\boldsymbol{Z}(\mathfrak{P}))\right)$. Hence, 1 is the only element in $И\left(\Omega_{1}(Z(\Re)) ; 3^{\prime}\right)$. Thus, if $\Re$ is a 3 -solvable subgroup of $\mathbb{B}$ and $S_{3}$ subgroups of $\Re$ are noncyclic, then $\Re$ is 3-closed. This implies by definition of $\sim$ that $\boldsymbol{A}_{\mathscr{G}}\left(\Omega_{1}(\boldsymbol{Z}(\mathfrak{P}))\right)$ contains an abelian subgroup of type $(4,2)$ or an elementary subgroup of order 8 . Neither of these possibilities holds in Aut $\left(\Omega_{1}(\boldsymbol{Z}(\mathfrak{P}))\right)$. Hence, $\mathscr{S}_{\mathrm{cn}_{3}}(3) \neq \varnothing$. We have shown that (i), (ii), (iii), (iv) of Hypothesis 7.4 hold. If $\mathfrak{A} \in \mathscr{S}_{\text {cns }_{3}}(2)$, then $И(\mathfrak{A})$ contains only 1 by Hypothesis (vi) of Theorem 8.1. Suppose $\mathfrak{A} \in \mathscr{S}_{\text {crn }_{3}}(3)$, and $\mathfrak{Q} \in \boldsymbol{U}(\mathfrak{U}), \mathfrak{\Omega} \neq 1, \mathfrak{\Omega}$ minimal with these properties. Let $\mathfrak{P}$ be a $S_{3}$-subgroup of $N(\mathfrak{A})$. Since $Z(\mathfrak{P})$ is noncyclic, we may choose $Z$ in $\boldsymbol{C}(\mathfrak{\Omega}) \cap \boldsymbol{Z}(\mathfrak{P})^{\ddagger}$. It follows that $\mathfrak{Q} \subseteq \boldsymbol{O}_{3^{\prime}}(\boldsymbol{C}(Z))$ against Hypothesis (i) of Theorem 8.1. (i) is proved.

Hypothesis 7.1 follows from Hypothesis 7.4 since if $p=2$ or 3 and $\mathfrak{B} \in \mathscr{U}^{*}(p)$, then $C(\mathfrak{B})$ contains an element of $\operatorname{Sen}_{3}(p)$.

In the remainder of this section, $\mathfrak{P}$ denotes a $S_{3}$-subgroup of (S), and $\mathfrak{B} \in \mathscr{U}(\mathfrak{P})$.

Let $\mathfrak{B}_{i}, 1 \leqq i \leqq 4$, be the subgroups of $\mathfrak{B}$ of order 3 . Let $\mathfrak{N}_{i}=$ $N\left(\mathfrak{B}_{i}\right)$, let $\mathfrak{D}_{i}=\mathfrak{B}^{\mathfrak{R}_{i}}$ and let $\mathfrak{E}_{i}=C_{\mathfrak{R}_{i}}\left(\mathfrak{D}_{i}\right)$. Since $3 \in \pi_{4}$ and $\mathfrak{P} \subseteq \mathfrak{R}_{i}$, we have $O_{3^{\prime}}\left(\mathfrak{R}_{i}\right)=1$. Hence, by Lemma 5.10, $\mathfrak{D}_{i}$ is 3-reducible in $\mathfrak{R}_{i}$. Finally, let $\mathfrak{R}_{i}=\mathfrak{N}_{i} / \mathfrak{S}_{i}$. Thus, $\mathfrak{R}_{i}$ may be identified with a subgroup of Aut $\left(\mathfrak{D}_{i}\right), \mathfrak{Q}_{i} \cong \boldsymbol{A}_{\mathfrak{N}_{i}}\left(\mathfrak{D}_{i}\right)$, and as such $\mathfrak{Q}_{i}$ is a 3 -solvable group with no nontrivial normal 3-subgroups. We let $\Re_{i}=O^{3^{\prime}}\left(\mathfrak{L}_{i}\right)$, so that $\Re_{i}$ is that subgroup of $\mathfrak{R}_{i}$ generated by the 3-elements of $\mathfrak{L}_{i}$.

The following lemma is important.

Lemma 8.5. Suppose for some $i, 1 \leqq i \leqq 4, \Re_{i}$ contains an element of order 3 which centralizes a subgroup of $\mathfrak{D}_{i}$ of index 3 . Then

(a) $\left|\mathfrak{D}_{i}\right|=27$.

(b) $\Re_{i} \cong S L(2,3)$.

(c) $\mathfrak{D}_{i}=\mathfrak{B}_{i} \times \mathfrak{F}_{i}$, where $\mathfrak{F}_{i} \triangleleft \mathfrak{R}_{i}$.

(d) $\Re_{i}$ is faithfully and irreducibly represented on $\mathfrak{F}_{i}$.

Proof. Let $\mathfrak{U}$ be the set of 3 -elements of $\mathfrak{N}_{i}$ which centralize some subgroup of index 3 in $\mathfrak{D}_{i}$. Since $\mathfrak{D}_{i} \triangleleft \mathfrak{N}_{i}, \mathfrak{U}$ is an invariant subset of $\mathfrak{N}_{i}$. By hypothesis, $\mathfrak{U}-\mathfrak{C}_{i} \neq \varnothing$.

Let $\mathfrak{U}^{*}=\mathfrak{U} \cap \mathfrak{P}$, and let $\mathfrak{U}_{1}=\left\langle U \mid U \in \mathfrak{U}^{*}\right\rangle$. For any subset $\mathfrak{S}$ of $\mathfrak{N}_{i}$, let $\overline{\mathfrak{F}}=\mathfrak{S C C}_{i} / \mathfrak{C}_{i}$. Since $\mathfrak{Q}_{i}$ is 3 -reduced, so is $\mathfrak{R}_{i}$. Furthermore, if $U \in \mathfrak{U}-\mathfrak{S}_{i}$, then $\bar{U}$ is an exceptional element in the sense of HallHigman [26, p. 10], or as we might say, an exceptional element, being the identity on a hyperplane of $\mathfrak{D}_{i}$. (In a perhaps more frequently used terminology, $\bar{U}$ is a transvection.)

Let $\mathfrak{S}=O_{3^{\prime}}\left(\mathfrak{R}_{i} \bmod \mathfrak{C}_{i}\right)$, so that $\overline{\mathfrak{U}}_{1}$ is faithfully represented on $\overline{\mathfrak{K}}$. 
By $(B), \overline{\mathfrak{U}}_{1}$ centralizes some $S_{2^{\prime}}$-subgroup of $\overline{\mathfrak{F}}$. Let $\bar{\Re}=\left[\overline{\mathscr{F}}, \overline{\mathfrak{U}}_{1}\right]$. Since $\overline{\mathscr{A}}$ is solvable, it follows that $\overline{\mathscr{\Omega}}$ is a $\overline{\mathscr{S}}$-invariant 2-group on which $\mathfrak{U}_{1}$ is faithfully represented, and that $\bar{\Omega}=\left[\bar{\AA}_{1}, \overline{\mathfrak{U}}_{1}\right]$. By Lemma 5.17, $\bar{\Omega}$ is special, since by $(B), \overline{\mathfrak{U}}_{1}$ centralizes every abelian $\overline{\mathfrak{U}}_{1}$-invariant subgroup of $\bar{\AA}$.

It may now be verified that if $U \in \mathfrak{U}^{*}-\mathfrak{S}_{i}$ and $\mathfrak{B}=\langle U\rangle$, then $[\bar{\Re}, \overline{\mathfrak{B}}]$ is a quaternion group and $[\bar{\Re}, \mathfrak{B}]$ centralizes a subgroup $\mathfrak{F}$ of index 9 in $\mathscr{S}_{i}$. Furthermore, $\mathfrak{D}_{i}=\mathfrak{F}_{i} \times \mathfrak{F}$, where $\mathfrak{F}_{i}=\left[\Re, \mathfrak{B}, \mathfrak{D}_{i}\right]$ is of order 9 , and $\mathfrak{F}_{i 0}=\left[\mathfrak{F}_{i}, \mathfrak{S}\right]$ is of order 3 . Since $\mathfrak{F}_{i}$ and $\mathfrak{F}$ are $\mathfrak{U}$-invariant, and since $U$ centralizes some hyperplane of $\mathfrak{D}_{i}$, it follows that $C_{\mathscr{D}_{i}}(U)=$ $\mathfrak{F}_{i 0} \times \Im$.

Let $\overline{\mathfrak{P}}_{1}=\overline{\mathrm{P}} \cap C(\overline{\mathfrak{R}})$ and let $\overline{\mathbb{Q}}=\overline{\mathfrak{R}} \bar{\AA}$. Since $\overline{\mathrm{Q}}$ is faithfully represented on $\mathfrak{D}_{i}$, so is its subgroup $\overline{\mathbb{P}_{1}} \bar{\Re}=\bar{\Re}_{1} \times \bar{\AA}$. Hence, by Lemma 3.7 of [20], $\overline{\mathfrak{R}}$ is faithfully represented on $\widetilde{\mathfrak{D}}_{i}=C_{\mathscr{D}_{i}}\left(\mathfrak{P}_{1}\right)$. Since $\overline{\mathfrak{P}} / \overline{\mathfrak{P}}_{1}$ is faithfully represented on $\bar{\AA}$, it follows that $\bar{\Sigma} / \overline{\mathfrak{P}}_{1}$ is faithfully repre-

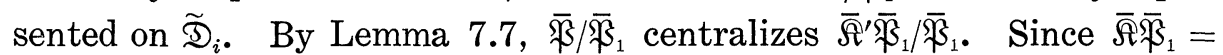
$\bar{\Re} \times \overline{\mathfrak{R}}_{1}$, it follows that $\overline{\mathfrak{R}}$ centralizes $\overline{\mathfrak{A}^{\prime}}$.

Since $\overline{\mathfrak{U}}_{1}$ is faithfully represented on $\bar{\AA} / \overline{\mathscr{\Omega}}^{\prime}$, and since each element $\overline{\mathfrak{U}}^{*}$ centralizes a subgroup of $\overline{\mathfrak{K}} / \overline{\mathfrak{K}}^{\prime}$ of index 4 , it is straightforward to verify that $\overline{\mathfrak{U}}_{1}$ is elementary. It then follows that every element of $\overline{\mathfrak{U}}_{1}^{*}$ is exceptional (though we don't contend that every element of $\overline{\mathfrak{U}}_{1}$ centralizes a hyperplane of $\left.\mathfrak{D}_{i}\right)$.

The preceding paragraph, together with $\left[\overline{\mathfrak{\Re}^{\prime}}, \overline{\mathfrak{P}}\right]=1$ and Corollary 2 of $\S 2.6$ of [24] imply that $\overline{\mathfrak{U}}_{1} \subseteq Z(\overline{\mathfrak{P}})$. Returning to $\mathfrak{B}$, we see that $[\overline{\mathfrak{K}}, \overline{\mathfrak{B}}]$ is $\overline{\mathfrak{P}}$-invariant. This in turn implies that $\mathfrak{F}$ is $\mathfrak{P}$-invariant. If $|\mathfrak{F}| \geqq 9$, then $\mathfrak{F}$ contains an element of $\mathscr{U}^{*}(3)$ and Lemma 7.4 is violated. Hence, $|\mathfrak{F}|<9$. Since $\mathfrak{B}_{i} \subseteq \mathfrak{F}$, we see that (a) and (c) hold. By construction, (b) and (d) follow. The proof is complete.

$J$ denotes the subset of $\{1,2,3,4\}$ whose elements satisfy the hypothesis of Lemma 8.5.

Lemma 8.6. Let $i \in J$ and let $\mathfrak{U}$ be a subgroup of $\mathfrak{F}_{i}$ of order 3 . Let $\mathfrak{N}=N(\mathfrak{R})$, let $\tilde{\mathfrak{R}}$ be the normal closure of $\mathfrak{B}_{i}$ in $\mathfrak{N}$ and $\tilde{\mathfrak{N}}_{1}$ be the normal closure of $\mathfrak{D}_{i}$ in $\mathfrak{N}$. Then

(a) $\left[\tilde{\mathfrak{N}}, \tilde{\mathfrak{N}}_{1}\right]=1$.

(b) $\left[\tilde{\mathfrak{R}}_{1}, \tilde{\mathfrak{A}}_{1}\right] \subseteq \mathfrak{A}$.

Proof. Let $\mathfrak{A}^{*}=\mathfrak{A} \times \mathfrak{B}_{i}$. Since the subgroups of $\mathfrak{F}_{i}$ of order 3 are permuted transitively in $\mathfrak{N}_{i}$, it follows that $C_{\mathfrak{N}_{i}}\left(\mathfrak{X}^{*}\right)$ contains a $S_{3^{-}}$ subgroup $\mathfrak{P}^{*}$ of $\mathbb{S}$. Thus, $\mathfrak{B}_{i}$ is contained in the center of a $S_{3}$-subgroup of $\mathfrak{R}$, namely $\mathfrak{P}^{*}$. By Lemma $5.10, \tilde{\mathfrak{R}}$ is 3-reducible in $\mathfrak{R}$. Since $\mathfrak{P}^{*} \subseteq \boldsymbol{C}_{\mathscr{H}}(\mathfrak{U})$ and $3 \in \pi_{4}$, we have $\boldsymbol{O}_{3^{\prime}}\left(\boldsymbol{C}_{\mathscr{H}}(\mathfrak{U})\right)=1$, which implies that $\boldsymbol{O}_{3^{\prime}}\left(\boldsymbol{C}_{\mathscr{G}}(\mathfrak{A}) / \mathfrak{A}\right)=1$. Since $\mathfrak{D}_{i} / \mathfrak{A} \subseteq \boldsymbol{Z}\left(\mathfrak{P}^{*} / \mathfrak{A}\right)$, we conclude that 


$$
\mathfrak{D}_{i} / \mathfrak{A} \subseteq \boldsymbol{O}_{3}\left(C_{\mathscr{S}}(\mathfrak{U}) / \mathfrak{A}\right) \subseteq O_{3}(\mathfrak{N} / \mathfrak{A}),
$$

and so $\mathfrak{D}_{i} \subseteq C_{\mathfrak{R}}(\tilde{\mathfrak{N}})$, by 3-reducibility of $\tilde{\mathfrak{R}}$ in $\mathfrak{R}$. Since $\boldsymbol{C}_{\mathfrak{R}}(\tilde{\mathfrak{N}}) \triangleleft \mathfrak{N}$, we have $\left[\tilde{\mathfrak{N}}, \tilde{\mathfrak{N}}_{1}\right]=1$. Since $\mathfrak{D}_{i} / \mathfrak{A} \subseteq Z\left(\boldsymbol{O}_{3}(\mathfrak{N} / \boldsymbol{A})\right)$, we also have $\left[\tilde{\mathfrak{N}}_{1}, \tilde{\mathfrak{N}}_{1}\right] \subseteq \mathfrak{A}$.

We now set $\mathfrak{D}=\left\langle\mathfrak{D}_{1}, \mathfrak{D}_{2}, \mathfrak{D}_{3}, \mathfrak{D}_{4}\right\rangle$. Since $\mathfrak{B} \subseteq Z(\mathfrak{P})$, it is clear that $\mathfrak{B} \subseteq \boldsymbol{Z}(\mathfrak{D})$ and that $\mathfrak{D} \triangleleft \mathfrak{P}$.

HYPoTHESIS 8.1. ( i )

$$
\begin{aligned}
& \mathfrak{P} \leqq \mathfrak{M} \in \mathscr{L} S(\mathfrak{S}), \mathfrak{B}=\Omega_{1}(\boldsymbol{Z}(\mathfrak{P}))^{\mathfrak{M}}, \\
& \mathfrak{S}=\boldsymbol{C}_{\mathfrak{M}}(\mathfrak{B}), \mathfrak{S}^{*}=V\left(\operatorname{ccl}_{\mathfrak{S}}(\mathfrak{D}) ; \mathfrak{P}\right) .
\end{aligned}
$$

(ii) $\mathfrak{B}^{*} \nsubseteq \mathfrak{C}$.

The long argument to follow is carried out under Hypothesis 8.1.

Choose $G$ in $\mathbb{B}$ so that $\mathfrak{D}^{G} \subseteq \mathfrak{P}$ but $\mathfrak{D}^{G} \nsubseteq \mathbb{C}$. The element $G$ plays a passive but important role. If $\mathfrak{S}$ is any subset of $\mathbb{S}$, we set $\mathfrak{S C}^{\cdot}=\mathfrak{S}^{G}$, while if $\mathfrak{S}$ is any subset of $\mathfrak{M}$, we set $\overline{\mathfrak{K}}=\mathfrak{S C E} / \mathbb{C}$.

Let $\Re$ be any subgroup of $\boldsymbol{O}_{3^{\prime}}(\overline{\mathfrak{M}})$ which admits $\mathfrak{D} \cdot$ and is minimal subject to $[\overline{\mathfrak{D}} \cdot, \mathfrak{R}] \neq 1$. (Notice that $\Re$ is available.) Let $N=N(\Re)=$ $\left\{i \mid 1 \leqq i \leqq 4, \overline{\mathfrak{D}}_{i}\right.$ does not centralize $\left.\boldsymbol{C}_{\mathfrak{R}}\left(\overline{\mathfrak{B}}_{i}\right)\right\}$. We argue that $N \neq \varnothing$. This is clear if $\overline{\mathfrak{B}} \cdot$ centralizes $\Re$, so we may assume that $[\overline{\mathfrak{B}} \cdot, \Re] \neq 1$. Since $\mathfrak{B}^{\cdot}$ is noncyclic, it follows that $\mathfrak{R}=\left\langle\mathfrak{R} \cap \boldsymbol{C}\left(\mathfrak{B}_{i}\right) \mid 1 \leqq i \leqq 4\right\rangle$, so we can choose $i$ such that $\overline{\mathfrak{D}} \cdot$ does not centralize $\boldsymbol{C}_{\Re}\left(\overline{\mathfrak{B}}_{i}\right)$. Minimality of $\mathfrak{R}$ guarantees that $\Re=C_{\mathfrak{R}}\left(\overline{\mathfrak{B}}_{i}\right)$. Thus, $\mathfrak{B}$. does not centralize $C_{\Re}\left(\overline{\mathfrak{B}}_{i}\right)$. Since $\mathfrak{B}^{\bullet} \cong \mathfrak{D}_{i}$, we have $i \in N(\Re)$. In the following discussion, $\Re$ is a fixed subgroup of $\boldsymbol{O}_{3^{\prime}}(\overline{\mathfrak{M}})$ which admits $\mathfrak{D} \cdot$ and is minimal subject to $[\overline{\mathfrak{D}} \cdot, \mathfrak{R}] \neq 1$, and $j$ is a fixed element of $N(\mathfrak{R})$. As already observed, $\overline{\mathfrak{B}}_{j}$ centralizes $\Re$.

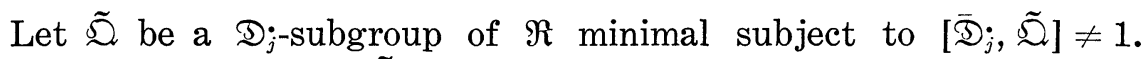
Let $\mathfrak{D}_{0}=\operatorname{ker}\left(\mathfrak{D}_{j} \rightarrow \operatorname{Aut}(\tilde{\mathfrak{\Omega}})\right)$, so that $\left|\mathfrak{D}_{j}: \mathfrak{D}_{0}\right|=3$ and $\mathfrak{B}_{j} \subseteq \mathfrak{D}_{0}$.

Since $\tilde{\mathfrak{Q}}$ is faithfully represented on $\mathfrak{B}$, Lemma 3.7 of [18] implies that $\tilde{\mathfrak{Q}}$ faithfully represented on $C_{\mathfrak{B}}\left(\mathfrak{D}_{0}^{*}\right)$. Since $\mathfrak{D}_{j}$ does not centralize $\boldsymbol{C}_{\mathfrak{B}}\left(\mathfrak{D}_{0}^{*}\right)$, we may choose $V$ in $\mathfrak{D}_{\mathfrak{B}}\left(\mathfrak{D}_{0}^{*}\right)-\boldsymbol{C}\left(\mathfrak{D}_{j}^{*}\right)$. Then

$$
V \in C\left(\mathfrak{B}_{j}\right) \subseteq N\left(\mathfrak{B}_{j}\right)=\mathfrak{R}_{j}
$$

Thus, $G V G^{-1}$ is a 3 -element of $\mathfrak{R}_{j}-\mathfrak{C}_{j}$ which centralizes a subgroup of $\mathfrak{D}_{j}$ of index 3. By Lemma 8.5,

$$
j \in J,\left|\mathfrak{D}_{j}\right|=27, \cdots .
$$

This implies that $\left|\boldsymbol{C}_{\mathfrak{B}}\left(\mathfrak{D}_{0}^{*}\right): \boldsymbol{C}_{\mathfrak{B}}\left(\mathfrak{D}_{j}^{*}\right)\right|=3$, which in turn implies that $\tilde{\mathfrak{I}}$ is a quaternion group.

Since $\widetilde{\mathfrak{Q}}$ is a quaternion group, the following assertions hold: 
(a) $\mathfrak{D} \cdot$ centralizes a $S_{2^{\prime}}$-subgroup of $\boldsymbol{O}_{3^{\prime}}(\overline{\mathfrak{M}})$.

(b) $\mathfrak{D} \cdot$ centralizes every abelian subgroup of $\boldsymbol{O}_{3^{\prime}}(\overline{\mathfrak{M}})$ which $\mathfrak{D} \cdot$ normalizes.

( c ) If $\mathfrak{F}$ is the normal closure in $\mathfrak{P}$ of $\mathfrak{D} \cdot$, then $\left[\overline{\mathfrak{F}}, \boldsymbol{O}_{3},(\mathfrak{M})\right]=\overline{\mathfrak{F}}$ is a special 2-group whose derived group is centralized by $\bar{\mho}$. Namely, if either (a) or (b) were false, we could find $\Re$ such that $\Re$ contains no quaternion group. Since this is not the case, (a) and (b) hold. Now (c) follows from Lemma 5.17, together with the solvability of $\boldsymbol{O}_{3},(\overline{\mathfrak{M}})$. We retain the previous notation and continue the argument.

Let $\mathfrak{A} \cdot=\left[\boldsymbol{C}_{\mathfrak{B}}\left(\mathfrak{D}_{0}\right), \mathfrak{D}_{j}\right]$. Thus, $\mathfrak{A} \cdot$ is a subgroup of $\mathfrak{\complement}_{j}$ of order 3 . Let $\mathfrak{B}=\mathfrak{B}_{0} \times \mathfrak{B}_{1}$, where $\mathfrak{B}_{0}=\boldsymbol{C}_{\mathfrak{B}}(\tilde{\mathfrak{D}}), \mathfrak{B}_{1}=[\mathfrak{B}, \tilde{\mathfrak{D}}]$. Since $\mathfrak{X} \cdot \subseteq \mathfrak{B}$, we have $\mathfrak{B} \subseteq N(\mathfrak{A} \cdot)=\mathfrak{R} \cdot$, so that $\left[\mathfrak{B}, \mathfrak{B}_{j}\right] \subseteq \mathfrak{B}_{j}^{\cdot \mathfrak{R}}$. By Lemma 8.6, $\left[\mathfrak{B}, \mathfrak{B}_{i}, \mathfrak{D}_{i}\right]=1$. This implies that $\tilde{\mathfrak{Q}}$ centralizes $\left[\mathfrak{B}, \mathfrak{B}_{j}\right]$, which in turn implies that $\left[\mathfrak{B}, \mathfrak{B}_{j}\right] \subseteq \mathfrak{B}_{0}$. Hence, $\mathfrak{S}_{j}$ centralizes $\mathfrak{B}_{1}$. Hence, $\mathfrak{D}_{j}$ centralizes $\left[\mathfrak{B}_{1}, \mathfrak{D}_{0}^{*}\right]$. As $\tilde{\mathfrak{O}}$ normalizes $\left[\mathfrak{B}_{1}, \mathfrak{D}_{0}^{*}\right]$, it follows that $\tilde{\mathfrak{Q}}$ centralizes $\left[\mathfrak{B}_{1}, \mathfrak{D}_{j}^{*}\right]$. By definition of $\mathfrak{B}_{1}$, we get $\left[\mathfrak{B}_{1}, \mathfrak{D}_{0}^{*}\right]=1$. Hence, $\left[\mathfrak{B}_{1}, \mathfrak{D}_{j}^{*}\right]=$ $\mathfrak{2} \cdot$ and $\left|\mathfrak{B}_{1}\right|=9$.

Suppose $\overline{\mathfrak{P}}$ centralizes $\overline{F^{\prime}}$. Since $\tilde{\mathfrak{Q}} \subseteq \overline{\mathfrak{F}}$, it follows that $\overline{\mathfrak{P}}$ centralizes $\tilde{\mathfrak{\Omega}}^{\prime}$, a group of order 2. Hence, $\overline{\mathfrak{P}}$ normalizes $\mathfrak{B}_{0}=C_{\mathfrak{S}^{\prime}}\left(\tilde{\mathfrak{\Omega}}^{\prime}\right)$. Since the inverse image of $\widetilde{\mathfrak{\Omega}^{\prime}}$ in $\mathfrak{M}$ contains an involution, it follows that $\mathfrak{B}_{0}$ contains no element of $\mathscr{U}^{*}(3)$. But $\mathfrak{B}_{0} \triangleleft \mathfrak{P}$, so the only possibility is that $\mathfrak{B}_{0}$ is cyclic. Since $\boldsymbol{Z}(\mathfrak{P})$ is non cyclic, we get $\left|\mathfrak{B}_{0}\right|=3$.

Suppose $\overline{\mathfrak{P}}$ does not centralize $\overline{\mathfrak{F}^{\prime}}$. Let $\overline{\mathfrak{F}_{1}}=\left[\overline{\mathfrak{F}^{\prime}}, \overline{\mathfrak{P}}\right]$, and let $\mathfrak{W}$ be a subgroup of $\mathfrak{B}$ which admits $\overline{\mathfrak{B}} \overline{\mathbb{F}}$, and is minimal subject to $\left[\overline{\mathfrak{S}}_{1}, \mathfrak{W}\right] \neq 1$. Since $\overline{\mathfrak{F}}$ centralizes $\overline{\mathbb{S}^{\prime}}$, it follows that $\overline{\mathfrak{F}}$ centralizes $\mathfrak{W}$; so $\mathfrak{D} \cdot$ centralizes $\mathfrak{W}$. Hence, $\mathfrak{W} \subseteq \mathfrak{B}_{0} \times \mathfrak{U} \cdot$. By Lemma 4.4 of [19], $\mathfrak{W}$ contains a subgroup $\mathfrak{W}_{0}$ of order 27 such that $\mathfrak{W}_{0} \triangleleft \mathfrak{P},\left|\mathfrak{B}: C_{\mathfrak{B}}\left(\mathfrak{W}_{0}\right)\right|=$ 3. Since $|\mathfrak{H} \cdot|=3$, it follows that $\mathfrak{B}_{0} \cap \mathfrak{W}_{0}$ is noncyclic. Let $\mathfrak{W}_{1}$ be a subgroup of $\mathfrak{B}_{0} \cap \mathfrak{W}_{0}$ of order 9 . Since $|\mathfrak{P}|$ is clearly larger than $3^{4}$, we conclude from Lemma 7.6 (d) that $\mathfrak{W}_{1} \in \mathscr{E}(3)$. Let $I$ be an involution in the inverse image of $\tilde{\mathfrak{a}}$ in $\mathfrak{M}$; then $I$ centralizes $\mathfrak{B}_{0}$, so centralizes $\mathfrak{W}_{1}$. Hence, by Lemma 7.4, $C(I)$ is nonsolvable. This contradiction shows that $\left[\overline{\mathfrak{P}}, \overline{\mathfrak{S}^{\prime}}\right]=1$. Hence, $\left|\mathfrak{B}_{0}\right|=3$, an important equality.

Since $\mathfrak{M} \in \mathbb{L C S}(G)$, it follows that $\mathfrak{M}=N\left(\mathfrak{B}_{0}\right)$, so that $\mathfrak{M}=\mathfrak{N}_{i}$ for some $i, 1 \leqq i \leqq 4$. Thus, $i \in J, \mathfrak{B}_{0}=\mathfrak{B}_{i}, \mathfrak{B}_{1}=\mathfrak{F}_{i}, \mathfrak{B}=\mathfrak{D}_{i}, \mathfrak{c}=\mathfrak{C}_{i}$.

Let $\mathfrak{X}_{0}=\mathfrak{P} \cap \mathfrak{S}_{i}, \mathfrak{R}_{0}=N_{\mathfrak{N}_{i}}\left(\mathfrak{P}_{0}\right)$, so that $\mathfrak{N}_{0} \mathfrak{S}_{i}=\mathfrak{N}_{i}$. Let $\mathfrak{\Omega}_{0}$ be a $S_{2}$-subgroup of $\mathfrak{R}_{0}$ permutable with $\mathfrak{P}$. Let $\mathfrak{S}=\mathfrak{S} \mathfrak{Q}_{0} \cap \mathbb{C}\left(\mathfrak{B}_{i}\right)$, and set $\mathfrak{Q}=\subseteq \cap \mathfrak{D}_{0}$. Then $\subseteq=\mathfrak{P} \mathfrak{}$.

Since $i \in J$, Lemma 8.5 implies that a $S_{2,3}$-subgroup of $\mathfrak{N}_{i} / \mathfrak{S}_{i}$ is not 3-closed. Since $\mathfrak{P D}_{0}$ is not 3 -closed, neither is $\mathfrak{S}$, since $\left|\mathfrak{B}_{0}: \mathfrak{S}\right| \leqq 2$. Let $I$ be an involution of $\Omega$. Suppose $\mathfrak{D}_{i} \cap \boldsymbol{C}(I) \supset \mathfrak{B}_{i}$. Then $\mathfrak{D}_{i} \cap \boldsymbol{C}(I)$ contains a subgroup $\widetilde{\mathfrak{D}}$ of order 9 with $\widetilde{\mathfrak{D}} \supset \mathfrak{B}_{i}$. Since $\mathfrak{S}$ permutes transitively the subgroups of $\widetilde{F}_{i}$ of order 3 , it follows that $\widetilde{D}$ is central 
in some $S_{3}$-subgroup of $\mathfrak{S}$, that is, $I$ centralizes an element of $\mathscr{U}(3)$. This is not the case, since $C(I)$ contains an element of $\mathscr{C}(2)$. This contradiction forces $\mathfrak{D}_{i} \cap C(I)=\mathfrak{B}_{i}$ for all involutions $I$ of $\mathfrak{\Omega}$. Since $\mathfrak{P} 丸 \mathfrak{S}, \mathfrak{D}$ is not cyclic. Thus, $\mathfrak{Q}$ is a quaternion group. Also, $\mathfrak{P}_{0}=$ $\boldsymbol{O}_{3}(\mathfrak{S})=\mathfrak{S} \cap \mathfrak{S}_{i}$ and $\mathfrak{S} / \mathfrak{P}_{0} \cong S L(2,3)$. In particular, $\mathfrak{B}_{j} \subseteq \mathfrak{P}_{0}$, while $\mathfrak{D}_{j} \nsubseteq \mathfrak{P}_{0}$.

Since $j \in J$, it follows that $\left[C_{\mathfrak{F}_{j}}\left(\mathfrak{B}_{j}\right)\right.$, $\left.\mathfrak{D}_{j}\right] \subseteq \mathfrak{F}_{j}$. Since $\mathfrak{B}_{j} \cong \mathfrak{P}_{0}$ and $\mathfrak{D}_{j} \nsubseteq \mathfrak{P}_{0}$, it follows that $\mathfrak{F}_{j} \nsubseteq \mathfrak{P}_{0}$. Hence, $\mathfrak{F}_{j} \cap \mathfrak{P}_{0}=\mathfrak{2} \cdot$. This implies that

$$
\left[C_{\mathfrak{B}_{0}}\left(\mathfrak{B}_{j}\right), \mathfrak{D}_{j}^{\cdot}\right]=\mathfrak{H} \cdot \text {. }
$$

For any subset $\mathfrak{T}$ of $\mathfrak{S}$, let $\mathbb{\mathfrak { T }}=\mathfrak{T} \mathfrak{F}_{i} / \mathfrak{F}_{i}$. It is important to show that

$$
C_{\bar{\Re}_{0}}\left(\overline{\mathfrak{B}}_{j}\right)=C_{\mathfrak{P}_{0}}\left(\mathfrak{B}_{j}\right) / \mathfrak{G}_{i} .
$$

Namely, suppose $P$ in $\mathfrak{P}_{0}$ satisfies $\left[\mathfrak{B}_{j}, P\right] \subseteq \mathfrak{F}_{i}$. Now $\quad \mathfrak{F}_{i}=\mathfrak{A} \cdot \times \mathfrak{A} *$, where $\mathfrak{X} \cdot$ and $\mathfrak{U}^{*}$ are of order 3 and $\mathfrak{X} \cdot \subseteq \mathfrak{F}_{j}$. We may apply Lemma 8.6 to $\mathfrak{N} \cdot$. Since $P \in \mathfrak{N} \cdot$, we get $\left[\mathfrak{B}_{j}, P, \mathfrak{D}_{j}^{\cdot}\right]=1$. Hence, $\left[\mathfrak{B}_{j}, P\right] \cong$ $\mathfrak{F}_{i} \cap \boldsymbol{C}\left(\mathfrak{D}_{j}\right)=\mathfrak{Y} \cdot$. Consider the group $\mathfrak{B}_{j} \times \mathfrak{A} \cdot \cdot$, which is normalized by the 3 -element $P$. Since $\mathfrak{N}_{j}$ permutes transitively the subgroups of $\mathfrak{F}_{j}$ of order 3 , it follows that $\mathfrak{B}_{j} \times \mathfrak{A} \cdot$ is in the center of some $S_{3}$-subgroup of $\mathfrak{s}$. Hence, $\boldsymbol{A}_{\mathscr{G}}\left(\mathfrak{B}_{j} \times \mathfrak{U} \cdot\right)$ is a $3^{\prime}$-group, so $P$ centralizes $\mathfrak{B}_{j} \times \mathfrak{A} \cdot$. We have proved (8.3).

Since $\mathfrak{A} \cdot \subseteq \mathfrak{F}_{i}$, so also $\mathfrak{P}_{0} \subseteq \mathfrak{N}$. Hence

$$
\left[\mathfrak{P}_{0}, \mathfrak{D}_{j}, \mathfrak{D}_{j}\right] \subseteq \mathfrak{U} \cdot
$$

by Lemma 8.6 (b) applied to $\mathfrak{N}$. We will use this fact several times. We next show that

$$
C_{\mathfrak{\$}_{0}}\left(\mathfrak{\Omega}^{\prime}\right)=C_{\mathfrak{\Re}_{0}}(\mathfrak{Q}) \text {. }
$$

Since $\mathfrak{C} \widetilde{\mathscr{F}}_{i}$ is a Frobenius group, it suffices to show that $C_{\overline{\$_{0}}}\left(\mathfrak{\Omega}^{\prime}\right)=$ $C_{\bar{\Re}_{0}}(\mathfrak{Q})$. Let $\mathscr{C}$ be part of a chief series of $\subseteq$ from $\mathfrak{P}_{0}$ to 1 , one of whose terms is $\mathfrak{F}_{i}$. If $\mathfrak{F}$ is a chief factor of $\mathscr{C}$, it suffices to show that if $\mathfrak{Q}^{\prime}$ centralizes $\mathfrak{F}$, so does $\mathfrak{\Omega}$. If this were not the case, then elements of $\mathfrak{D}_{j}^{-}-\mathfrak{P}_{0}$ would have minimal polynomial $(x-1)^{3}$ on $\mathfrak{F}$, against (8.4). Thus, (8.5) holds.

Suppose $\mathfrak{Z}^{\prime}$ centralizes $\overline{\mathfrak{P}}_{0}$. Let $\mathfrak{P}_{1}=C_{\mathfrak{F}_{0}}\left(\mathfrak{Q}^{\prime}\right)$. We get $\mathfrak{P}_{0}=\mathfrak{P}_{1}\left(\mathfrak{F}_{i}\right.$, $\mathfrak{P}_{1} \cap \mathfrak{F}_{i}=1$. Since $\mathfrak{F}_{i}$ is an irreducible $\mathfrak{Q}$-module, we have $\mathfrak{P}_{0}=\mathfrak{P}_{1} \times$ $\mathfrak{F}_{i}$. If $\mathfrak{P}_{1}$ is not cyclic, then $\mathfrak{Q}^{\prime}$ centralizes an element of $\mathscr{C}^{*}(3)$, which is not the case, since $\mathfrak{Q}^{\prime}$ centralizes an element of $\mathscr{U}(2)$. Thus, $\mathfrak{P}_{1}$ is cyclic. Clearly, $\mathfrak{P}_{1} \neq 1$, since $\mathfrak{B}_{i} \subseteq \mathfrak{P}_{1}$. If

$$
\left|\mathfrak{P}_{1}\right|>3, \text { then } \sigma^{1}\left(\mathfrak{P}_{1}\right) \triangleleft\left\langle\mathfrak{N}_{i}, \mathfrak{R}_{j}\right\rangle \text {, }
$$

while it is trivial that $\left\langle\mathfrak{N}_{i}, \mathfrak{R}_{j}\right\rangle$ is non solvable. Hence, $\mathfrak{P}_{1}=\mathfrak{B}_{i}$. But then Lemma 7.8 is violated. 
Let $\mathfrak{P}_{2}=\left[\mathfrak{P}_{0}, \mathfrak{\Omega}\right]$. By the preceding paragraph,

$$
\overline{\mathfrak{R}}_{2} \neq 1 \text {. }
$$

We will show that

$$
\overline{\mathfrak{P}}_{2} \text { is of exponent } 3 \text { and class at most } 2 \text {. }
$$

Let $\Re_{1}=\left[\mathfrak{P}_{2}, \mathfrak{D}_{j}^{\cdot}\right]$. Since $\mathfrak{P}_{2} \subseteq \Re \cdot$, Lemma $8.6(\mathrm{~b})$ implies that $\left[\Re_{1}, \Re_{1}\right] \subseteq$ $\mathfrak{2} \cdot$, so that $\bar{\Re}_{1}$ is abelian. Since $\mathfrak{D}_{j}$ is elementary so is $\bar{\Re}_{1}$. Thus $\bar{\Re}_{1}$

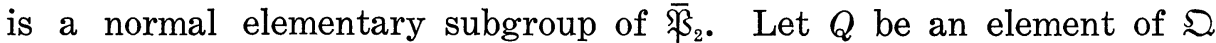
of order 4 , and set $\Re_{2}=\Re_{1}^{Q}$. We argue that $\bar{\Re}_{1} \bar{\Re}_{2}=\overline{\mathfrak{R}}_{2}$. To see this, observe that since $\mathfrak{\Omega}^{\prime}$ inverts $\mathfrak{\beta}_{2} / \boldsymbol{D}\left(\mathfrak{P}_{2}\right)$, and since the minimal polynomial of each element of $\mathfrak{D}_{j}$ on $\overline{\mathfrak{P}}_{2} / \boldsymbol{D}\left(\overline{\mathfrak{P}}_{2}\right)$ is a divisor of $(x-1)^{2}$, it follows that $\Re_{1}, \Re_{2}$ map onto subspaces of $\overline{\mathfrak{R}}_{2} / \boldsymbol{D}\left(\overline{\mathfrak{P}}_{2}\right)$ which generate $\overline{\mathfrak{P}}_{2} / \boldsymbol{D}\left(\overline{\mathfrak{F}}_{2}\right)$, so our assertion follows. Since $\bar{\Re}_{1}, \bar{\Re}_{2}$ are normal elementary subgroups of $\bar{\Re}_{2}$, (8.7) holds. Since we now have $\boldsymbol{D}\left(\bar{\Re}_{2}\right)=\left[\bar{\Re}_{1}, \bar{\Re}_{2}\right] \subseteq \bar{\Re}_{1}$, and since $\overline{\mathfrak{D}}_{j}^{*}$ centralizes $\bar{\Re}_{1}$, it follows that

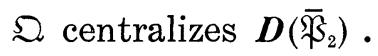

Since $\mathfrak{Q}$ has no fixed points on $\overline{\mathfrak{P}}_{2} / \boldsymbol{D}\left(\overline{\mathfrak{P}}_{2}\right)$, it follows from (8.4) that

$\mathfrak{Q}$ operates on $\overline{\mathfrak{P}}_{2} / \boldsymbol{D}\left(\overline{\mathfrak{P}}_{2}\right)$ as a multiple $d$ of the faithful irreducible $\mathfrak{\Omega}$-representation .

In particular,

$$
\left|\overline{\mathfrak{P}}_{2}: \boldsymbol{D}\left(\overline{\mathfrak{P}}_{2}\right)\right|=3^{2 d} \text {. }
$$

Let $B$ a generator for $\mathfrak{B}_{j}$, and for any element $S$ of $\mathfrak{S}$, let $S$ ] be the mapping of $\mathfrak{P}_{2}$ into itself which sends $P$ to $[P, S]$. We may view $B$ ] in more than one way. Since $\mathfrak{Q}$ centralizes $\mathfrak{P}_{0} / \mathfrak{P}_{2}$, we have $B=C U$, where $C \in C_{\mathfrak{P}_{0}}(\mathfrak{\Omega})$ and $U \in \mathfrak{P}_{2}$. Since $\left.U \in \mathfrak{P}_{2}, B\right]$ and $C$ ] induce the same mapping from $\mathfrak{P}_{2} / \boldsymbol{D}\left(\mathfrak{P}_{2}\right)$ to itself. In particular, $\left[\mathfrak{P}_{2}, B\right] \boldsymbol{D}\left(\mathfrak{P}_{2}\right)$ admits $\mathfrak{O}$. By Lemma 8.6 applied to $\mathfrak{N} \cdot$, we have $\left[\mathfrak{P}_{2}, B, \mathfrak{D}_{j}^{\cdot}\right]=1$. This implies that $\mathfrak{Q}$ centralizes $\left[\mathfrak{P}_{2}, B\right] \boldsymbol{D}\left(\mathfrak{P}_{2}\right) / \boldsymbol{D}\left(\mathfrak{P}_{2}\right)$, so by construction of $\mathfrak{P}_{2}$, we have

$$
\left[\mathfrak{P}_{2}, B\right] \subseteq \boldsymbol{D}\left(\mathfrak{P}_{2}\right) \text {. }
$$

Hence, $\left[\mathfrak{P}_{2}, C\right] \subseteq \boldsymbol{D}\left(\mathfrak{P}_{2}\right)$. Since $C$ centralizes $\cong$ and $\cong$ centralizes $\boldsymbol{D}\left(\overline{\mathfrak{P}}_{2}\right)$, we conclude that $C$ centralizes $\overline{\mathfrak{P}}_{2}$, by the three subgroups lemma. Hence, $B$ and $U$ induce the same automorphism of $\overline{\mathfrak{P}}_{2}$.

By Lemma 8.6 applied to $\Re \cdot, B$ centralizes the normal closure of $\mathfrak{D}_{j}^{*}$ in $\mathfrak{N} \cdot$. Hence, $\boldsymbol{C}_{\mathfrak{P}_{2}}(B) \supseteqq\left[\mathfrak{F}_{2}, \mathfrak{D}_{j}^{*}\right] \mathfrak{\xi}_{i} \mathfrak{P}_{2}^{\prime}$. We will show that

$$
C_{\mathfrak{P}_{2}}(B)=\left[\mathfrak{P}_{2}, \mathfrak{D}_{j}\right] \mathfrak{F}_{i} \mathfrak{P}_{2}^{\prime},
$$

$$
\left|\overline{\boldsymbol{C}_{\mathfrak{P}_{2}}(B)}: \boldsymbol{D}\left(\overline{\mathfrak{P}}_{2}\right)\right|=3^{d} \text {. }
$$


Let $\mathfrak{W}_{1}$ be the set of fixed points of $\mathfrak{D}_{j}$ on $\overline{\mathfrak{P}}_{2} / \boldsymbol{D}\left(\overline{\mathfrak{P}}_{2}\right)$, let

$$
\mathfrak{W}_{2}=\left[\overline{\mathfrak{D}_{j}^{*}, \mathfrak{P}_{2}}\right] \boldsymbol{D}\left(\overline{\mathfrak{F}}_{2}\right) / \boldsymbol{D}\left(\overline{\mathfrak{F}}_{2}\right) \text {, and let } W_{3}=\overline{\boldsymbol{C}_{\mathfrak{\beta}_{2}}(B)} / \boldsymbol{D}\left(\overline{\mathfrak{F}}_{2}\right) \text {. }
$$

From (8.2) and (8.3), we get that $\mathfrak{W}_{3} \subseteq \mathfrak{W}_{1}$. By Lemma 8.6 (a) applied to $\mathfrak{N} \cdot$, we get $\mathfrak{W}_{2} \subseteq \mathfrak{W}_{3}$. By (8.10) and Lemma 5.2, it follows that $\left|\mathfrak{W}_{1}\right| \leqq 3^{d}$. Using (8.10) once again, we get $\left|\mathfrak{W}_{2}\right| \geqq 3^{d}$. Since $\mathfrak{W}_{2} \subseteq$ $\mathfrak{W}_{3} \subseteq \mathfrak{W}_{1}$, it follows that $\mathfrak{W}_{1}=\mathfrak{W}_{2}=\mathfrak{W}_{3}$ is of order $3^{d}$. This yields (8.12) and (8.13).

Let $\mathfrak{B}^{*}=\mathfrak{B}_{j} \mathfrak{P}$. Since $\mathfrak{P}$ centralizes $\mathfrak{A} \cdot$, we have $\mathfrak{B}^{*} \subseteq \mathfrak{B}_{j} \mathfrak{\Re}$. By Lemma 8.6, $\mathfrak{B}^{*}$ and $\mathfrak{D}_{j}^{\cdot \mathfrak{P}}$ commute elementwise. Set $\mathfrak{P}_{3}=\left[\mathfrak{P}_{2}, \mathfrak{B}^{*}\right] \mathfrak{F}_{i} \triangleleft \mathfrak{P}$. Since $B$ centralizes $\overline{\mathfrak{P}}_{2} / \boldsymbol{D}\left(\overline{\mathfrak{P}}_{2}\right)$, (8.8) implies that $\cong$ normalizes $\mathfrak{P}_{3}$. Thus, $\mathfrak{P}_{3} \triangleleft \mathfrak{S}$. Since $\mathfrak{D}_{j}^{\cdot \mathfrak{P}}$ and $\mathfrak{B}^{*}$ commute elementwise, $\left[\mathfrak{P}_{2}, \mathfrak{D}_{j}^{*}\right.$ ] centralizes $\mathfrak{P}_{3}$. Hence, $\left[\mathfrak{P}_{2}, \mathfrak{D}_{j}^{*}\right]^{Q}$ centralizes $\mathfrak{P}_{3}^{Q}=\mathfrak{P}_{3}, Q$ being an element of $\mathfrak{\Omega}-\mathfrak{Q}^{\prime}$. Since $\mathfrak{P}_{2}=\left[\mathfrak{P}_{2}, \mathfrak{D}_{j}^{j}\right]\left[\mathfrak{P}_{2}, \mathfrak{D}_{j}^{*}\right]^{Q}$, it follows that $\mathfrak{P}_{2}$ centralizes $\mathfrak{P}_{3}$.

Let $\tilde{\mathfrak{P}}_{3}=\mathfrak{P}_{3} \cap C(\mathfrak{D})$. Thus, $\mathfrak{P}_{3}=\tilde{\mathfrak{F}}_{3} \times \mathfrak{F}_{i}$. Clearly, $N_{\Im}(\mathfrak{Q})$ normalizes $\tilde{\mathfrak{P}}_{3}$; so does $\mathfrak{P}_{2}$ since $\mathfrak{P}_{2}$ centralizes $\mathfrak{P}_{3}$. Since $\mathfrak{S}=\mathfrak{P}_{2} N_{\mathbb{S}}(\mathfrak{D})$, we have $\tilde{\mathfrak{P}}_{3} \triangleleft \mathfrak{S}$. Since $\mathfrak{Q}$ contains an involution, no subgroup of $\tilde{\mathfrak{P}}_{3}$ is in $\mathscr{Q}^{*}(\mathfrak{P})$. Hence, $\tilde{\mathfrak{P}}_{3}$ is cyclic. Since $\mathfrak{P}_{3}$ is isomorphic to a subgroup of $\overline{\mathfrak{P}}_{2}$, it follows that $\tilde{\mathfrak{R}}_{3}$ is of order 1 or 3 .

Suppose $\left[\mathfrak{P}_{2}, B\right] \subseteq \mathfrak{F}_{i}$. Then $(8.3)$ forces $\left[\mathfrak{P}_{2}, B\right]=1$. This violates (8.6), (8.10), (8.13). Thus, $\tilde{\mathfrak{P}}_{3}$ is of order 3 and $\mathfrak{P}_{3}=\left[\mathfrak{P}_{2}, B\right] \mathfrak{F}_{i}$, and $\left[\mathfrak{P}_{2}, B\right]$ is of order 3. Now (8.10) and (8.13) yield that $d=1$.

Suppose $\boldsymbol{D}\left(\overline{\mathfrak{P}}_{2}\right)=1$. Then by (8.11), $B$ centralizes $\overline{\mathfrak{P}}_{2}$. This conflicts with $(8.10)$ and (8.13). Hence, $\boldsymbol{D}\left(\overline{\mathfrak{P}}_{2}\right) \neq 1$, so that

$$
\left|\mathfrak{P}_{2}\right|=3^{5} \text {. }
$$

Since $\mathfrak{B}_{i} \tilde{\mathfrak{R}}_{3}$ is a normal subgroup of $\subseteq$ centralized by $\mathfrak{\Omega}$, we get $\mathfrak{B}_{i}=\tilde{\mathfrak{P}}_{3}$, as $\mathfrak{Q}$ centralizes no element of $\mathscr{C}^{*}(3)$. Hence,

$$
\boldsymbol{Z}\left(\mathfrak{P}_{2}\right)=\mathfrak{D}_{i} \text {. }
$$

Since $\mathfrak{B}_{2}$ is the normal closure of $\left[\mathfrak{P}_{2}, \mathfrak{D}_{j}^{*}\right]$ in $\mathfrak{S}$, and since $\mathfrak{D}_{j}^{\cdot \mathfrak{N}}$ is of exponent 3 , it follows that $\mathfrak{P}_{2}$ is generated by elements of order 3 . Since $\mathfrak{P}_{2}$ is of class 2 , it follows that

$$
\mathfrak{P}_{2} \text { is of exponent } 3 \text {. }
$$

Since $\mathfrak{B}_{i} \subset \mathfrak{P}_{2}$, the group $\mathfrak{P}_{2} / \mathfrak{B}_{i}$ is of order $3^{4}$ and is inverted by the involution of $\mathfrak{\Omega}$. Hence, $\mathfrak{P}_{2}^{\prime} \subseteq \mathfrak{B}_{i}$. Since $\mathfrak{P}_{2}$ is non abelian it follows that

$$
\mathfrak{P}_{2}^{\prime}=\mathfrak{B}_{i} \text {. }
$$

We next show that $B \in \mathfrak{P}_{2}$. Namely, $\mathfrak{B}=C U$, so that $[C, U]=$ $[C, C U]=[C, B]$. As we have already seen, $C$ centralizes $\overline{\mathfrak{P}}_{2}$, that is, $[C, U] \in \mathfrak{F}_{i}$. Since $[C, U]=[C, B],(8.3)$ implies that $[C, B]=1$. Since 
we have also shown that $\left[\mathfrak{P}_{2}, B\right]$ has order $3^{d}=3$, it follows that $U$

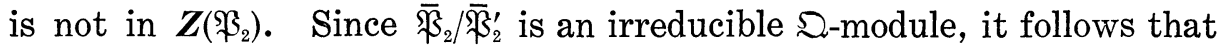
$C$ centralizes $\mathfrak{P}_{2}$, as $C$ centralizes an element of $\mathfrak{P}_{2}$ (namely, $U$ ) which does not map into $\overline{\mathfrak{P}}_{2}^{\prime}$. Since $C$ and $U$ commute, and since $B$ and $U$ have order 1 or 3 , it follows that $C$ has order 1 or 3 . If $C \notin \Re_{2}$, then $\Omega_{1}\left(C_{\mathfrak{P}_{0}}\left(\mathfrak{P}_{2}\right)\right) \cap C(\mathfrak{\Omega})$ is noncyclic, so that $\mathfrak{Q}$ centralizes an element of $\mathscr{U}^{*}(3)$. Since this is not the case, we conclude that

$$
B \in \mathfrak{P}_{2} \text {. }
$$

We will next show that $\mathfrak{P}_{0}=\mathfrak{P}_{2}$.

Since $B \in \mathfrak{P}_{2}$, $\left[\mathfrak{P}_{0}, B\right]$ is a subgroup of $\mathfrak{P}_{2}$ centralized by $\mathfrak{D}_{j}$. Suppose $\left[\mathfrak{F}_{0}, B\right] \nsubseteq \mathfrak{B}_{2}^{\prime}\left(=\mathfrak{B}_{i}\right)$. Let $B_{i}$ be a generator for $\mathfrak{B}_{i}, A$ a generator for $\mathfrak{V} \cdot$. Choose $P$ in $\mathfrak{P}_{0}$ so that $[P, B]=B_{i}{ }^{a} A^{b}$ with $b \neq 0$. Clearly, $a \neq 0$, since $\boldsymbol{A}_{\mathscr{S}}(\langle B, A\rangle)$ is a $3^{\prime}$-group. Since $\left[\mathfrak{P}_{2}, B\right]=\mathfrak{P}_{2}^{\prime}=\mathfrak{B}_{i}$, we may choose $P_{2}$ in $\mathfrak{P}_{2}$ so that $\left[P_{2}, B\right]=B_{i}^{-a}$. Then $\left[P P_{2}, B\right]=A^{b}$, which is impossible. Hence, $\left[\mathfrak{P}_{0}, B\right]=\mathfrak{P}_{2}^{\prime}$. Hence, $\left[\mathfrak{P}_{1}, \mathfrak{P}_{2}\right]=1$, by the three subgroups lemma. Here $\mathfrak{P}_{1}=\mathfrak{P}_{0} \cap \boldsymbol{C}(\mathfrak{Q})$. Hence, $\mathfrak{P}_{1} \triangleleft \mathfrak{S}$, so $\mathfrak{P}_{1}$ is cyclic, as $\mathfrak{Q}$ centralizes no element of $\mathscr{U}^{*}(3)$. If $\left|\mathfrak{P}_{1}\right|>3$, it is easy to verify that $\sigma^{1}\left(\mathfrak{P}_{1}\right) \triangleleft\left\langle\mathfrak{N}_{i}, \mathfrak{N}_{j}\right\rangle$ against the nonsolvability of $\left\langle\mathfrak{N}_{i}, \mathfrak{R}_{j}\right\rangle$. Hence, $\mathfrak{P}_{1}$ is of order 3 , so that $\mathfrak{P}_{1}=\mathfrak{B}_{i}$. Hence,

$$
\mathfrak{P}_{0}=\mathfrak{P}_{2} \text { is of order } 3^{5} \text {, }
$$

$\mathfrak{B}$ is of order $3^{6}$.

With the preceding information at our disposal, we turn our attention to $\mathfrak{M}=\mathfrak{N}_{i}$ once again. Let $\widetilde{\subseteq}$ be a $S_{\{2,3\}}$-subgroup of $\mathfrak{M}$ permutable with $\mathfrak{P}$. Then $\widetilde{\mathfrak{S}}$ centralizes $\mathfrak{D}_{i}$, for otherwise $2^{3} \cdot 3 \cdot 13$ divides $\boldsymbol{A}_{\mathfrak{M}}\left(\mathfrak{D}_{i}\right)$, forcing nonsolvability of $\boldsymbol{A}_{\mathfrak{M}}\left(\boldsymbol{D}_{i}\right)$. Since $\left|\boldsymbol{O}_{2}(\mathfrak{M}): \mathfrak{D}_{i}\right| \leqq$ 9, $\widetilde{\subseteq}$ also centralizes $\boldsymbol{O}_{3}(\mathfrak{M}) / \mathfrak{D}_{i}$. Hence, $\widetilde{\subseteq}$ centralizes $\boldsymbol{O}_{3}(\mathfrak{M})$, or equivalently,

$\mathfrak{M}$ is a 2,3 -group .

Let $\mathfrak{I}$ be a $S_{2}$-subgroup of $\mathfrak{N}_{i}$ containing $\mathfrak{\Omega}$. Since $\mathfrak{F}_{i} \in \mathscr{U}^{*}(\mathfrak{P})$, no element of $\mathfrak{I}^{\sharp}$ centralizes $\mathfrak{F}_{i}$. Thus,

$$
\mathfrak{P}_{0}=C\left(\mathfrak{F}_{i}\right) \text {. }
$$

Since $\mathfrak{B}_{i} \subseteq \mathfrak{B}$, it follows that $\boldsymbol{C}(\mathfrak{B}) \subseteq \mathfrak{N}_{i}=\mathfrak{M}$. By Lemma 7.4, $|\boldsymbol{C}(\mathfrak{B})|$ is odd. From (8.21) we conclude that

$$
\mathfrak{P}=C(\mathfrak{B}) \text {. }
$$

By construction, $\mathfrak{D}_{i} \subseteq \mathfrak{P}_{0}$. Suppose $j_{0}$ is an index such that $\mathfrak{D}_{j_{0}} \nsubseteq \mathfrak{P}_{0}$. Then $\left[\mathfrak{D}_{i}, \mathfrak{D}_{j_{0}}\right] \neq 1$. In this case, two applications of Lemma $8.5 \mathrm{imply}$ that $\left|\mathfrak{D}_{i}\right|=\left|\mathfrak{D}_{j_{0}}\right|=27$ and that $\left[\mathfrak{P}, \mathfrak{D}_{j_{0}}\right]$ is of order 3 . Hence, $\left[\mathfrak{P}, \mathfrak{D}_{j_{0}}\right]=$ 
$\left[\mathfrak{D}_{i}, \mathfrak{D}_{j_{0}}\right]$ so that $\mathfrak{D}_{j_{0}}$ centralizes $\mathfrak{P}_{0} / \mathfrak{D}_{i}$. This contradicts (8.6) and (8.19). Hence, no such $j_{0}$ exists, that is,

$$
\mathfrak{D} \subseteq \mathfrak{P}_{0} \text { • }
$$

Our previous information shows that $\boldsymbol{Z}(\mathfrak{P})=\mathfrak{B}$. Hence, $\boldsymbol{N}(\mathfrak{P})$ normalizes $\mathfrak{B}$, so permutes the groups $\mathfrak{B}^{N\left(\mathfrak{B}_{k}\right)}$ among themselves, $1 \leqq$ $k \leqq 4$. By definition of $\mathfrak{D}$, we get

$$
N(\mathfrak{P}) \subseteq N(D) \text {. }
$$

Suppose $\mathfrak{D} \cdot \triangleleft \mathfrak{P}$. Since $\mathfrak{D} \triangleleft \mathfrak{P}$, it follows that $\mathfrak{D} \cdot \triangleleft\langle\mathfrak{P}, \mathfrak{P} \cdot\rangle$. We can choose $N$ in $N\left(\mathfrak{D}^{\cdot}\right)$ so that $\mathfrak{P}^{\cdot N}=\mathfrak{P}$. Hence, $\mathfrak{D}^{\cdot N}=\mathfrak{D} \cdot$ and $\mathfrak{P}^{G N}=$ $\mathfrak{P}$. Let $H=G N$. Then $\mathfrak{D}^{\cdot}=\mathfrak{D}^{H}$ and $H \in N(\mathfrak{P})$. By (8.25), we get $\mathfrak{D}^{\cdot}=\mathfrak{D}^{H}=\mathfrak{D}$. This conflicts with (8.24), since by construction $\mathfrak{D} \cdot \nsubseteq \mathfrak{P}_{0}$. Thus,

$$
\mathfrak{D} \cdot 丸 \mathfrak{P} \text {. }
$$

Suppose $\mathfrak{B}^{*}$ is a $S_{3}$-subgroup of $\mathfrak{R}_{j}$ and that $\mathfrak{P}^{*} \subseteq \mathfrak{R}_{i}$. Thus, $\boldsymbol{Z}\left(\mathfrak{P}^{*}\right) \subseteq \mathfrak{D}_{i} \cap \mathfrak{D}_{j}^{+}=\mathfrak{A} \cdot$. This is impossible since $|\mathfrak{A} \cdot|=3,\left|\boldsymbol{Z}\left(\mathfrak{P}^{*}\right)\right|=9$. We conclude that

$$
\mathfrak{N}_{i} \cap \mathfrak{N}_{j} \text { contains no } S_{3} \text {-subgroup of } \$ \text { S . }
$$

Since $\mathfrak{D} \cdot \not \mathfrak{P},(8.20)$ implies that $|\mathfrak{D}| \leqq 3^{4}$. Suppose $|\mathfrak{D}| \leqq 3^{3}$. Then $\mathfrak{D} \supseteqq \mathfrak{D}_{i}$ implies $|\mathfrak{D}|=3^{3}$ and $\mathfrak{D}=\mathfrak{D}_{i}$. Thus, for each $k, 1 \leqq k \leqq 4$, we have $\mathfrak{B} \subseteq \mathfrak{D}_{k} \subseteq \mathfrak{D}_{i}$. If $\mathfrak{B}=\mathfrak{D}_{k}$, then $\mathfrak{N}_{k}$ normalizes $C(\mathfrak{B})$. By (8.23), we have $\mathfrak{P} \triangleleft \mathfrak{N}_{k}$, so by (8.25), we have $\mathfrak{R}_{k} \subseteq N(\mathfrak{D})$. Thus, if $|\mathfrak{D}|=3^{3}$, then $\left\langle\mathfrak{N}_{1}, \mathfrak{R}_{2}, \mathfrak{R}_{3}, \mathfrak{N}_{4}\right\rangle \subseteq N(\mathfrak{D})$. Since $\mathfrak{H} \cdot \subseteq Z(\mathfrak{P})$, it follows that $\mathfrak{P} \cdot=\mathfrak{B}_{k}$ for some $k$. Hence, $N(\mathfrak{X} \cdot) \subseteq \mathfrak{N}_{i}$. But $\mathfrak{Y} \cdot$ is a subgroup of $\mathfrak{D}_{j}$, so there is a $S_{3}$-subgroup $\mathfrak{B}^{*}$ of $\mathfrak{R}_{j}$ which contains $\mathfrak{A} \cdot$ in its center. This violates (8.27). Hence, $|\mathfrak{D}|=3^{4}$. Since $\mathfrak{P}_{0} \supset \mathfrak{D} \supset \mathfrak{D}_{i}$, we conclude that

(D) is elementary of order $3^{4}$.

Let $\mathfrak{C}$ be any subgroup of $\mathfrak{D}_{j}$ which is of order 3 and is not contained in $\mathfrak{B}_{0}$. Thus, $\mathfrak{D}_{j}=\mathfrak{C} \times \mathfrak{B}_{j} \times \mathfrak{A} \cdot$. Since $\mathfrak{D} \cdot \subseteq \mathfrak{P}$, it follows that $\mathfrak{D} \cdot \subseteq C_{\mathfrak{P}_{3}}\left(\mathfrak{D}_{j}^{j}\right)=\left(\mathfrak{S} \cdot C_{\mathfrak{P}_{0}}\left(\mathfrak{D}_{j}^{*}\right)\right.$, and as we have already shown, $\boldsymbol{C}_{\mathfrak{S}_{0}}\left(\mathfrak{B}_{j}\right)=$ $\mathfrak{D}_{i} \mathfrak{B}_{j}$ (that is, $\mathfrak{B}_{j} \nsubseteq Z\left(\mathfrak{P}_{0}\right)$ ). Since $\mathfrak{D}_{j}$ does not centralize $\mathfrak{F}_{i}$, it follows that $C_{\mathfrak{P}_{0}}\left(\mathfrak{D}_{j}^{\cdot}\right)=\mathfrak{B}_{j} \times \mathfrak{B}_{i} \times \mathfrak{U} \cdot$. Since $\mathfrak{D} \cdot \nless \mathfrak{F}$, it follows that $\boldsymbol{N}_{\mathfrak{\beta}}(\mathfrak{D} \cdot)=$ $\mathfrak{D} \cdot \mathfrak{F}_{i}$. Choose $P$ in $\mathfrak{P}_{0}-N_{\mathfrak{P}}(\mathfrak{D} \cdot)$. Since

$$
\left[P, \mathfrak{B}_{j}, \mathfrak{B}_{i} \mathfrak{A} \cdot\right] \subseteq \mathfrak{B}_{i} \subseteq \mathfrak{D} \cdot
$$

it follows that $[P, C] \notin \mathfrak{D} \cdot$, where $C$ is a generator for (5. Hence, $[P, C]=D E_{i}$ with $E_{i}$ in $\mathfrak{F}_{i}-\mathfrak{A} \cdot$ and $D$ in $\mathfrak{D} \cdot \cap \mathfrak{P}_{0}$. Hence, $[P, C, C]=$ $\left[D E_{i}, C\right]=\left[E_{i}, C\right]$ is a generator for $\mathfrak{A} \cdot$ This is a subtle and important 
bit of information, since it shows that the $\mathfrak{E}$ module $\mathfrak{P}_{0} / \mathfrak{P}_{0}^{\prime}$ has an indecomposable constituent of dimension 3. Thus,

the indecomposable direct factors of $\mathfrak{P}_{0} / \mathfrak{P}_{0}^{\prime}$ as 5 -modules are of dimensions 1 and 3 .

We note

$$
\mathfrak{P}=V\left(\operatorname{ccl}_{(S)}(\mathfrak{D}) ; \mathfrak{P}\right) \text {. }
$$

Namely, $\mathfrak{D}$ does not normalize $\mathfrak{D}, \mathfrak{D} \mathfrak{Q}=\mathfrak{P}_{0}$. Since $\mathfrak{P}=\left\langle\mathfrak{P}_{0}, \mathfrak{D} \cdot\right\rangle$, (8.30) holds. This fact has an important consequence. Namely, if

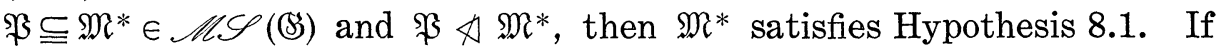
this were not so, then $\mathfrak{P} \leqq C_{\mathfrak{M}^{*}}\left(\Omega_{1}(\boldsymbol{Z}(\mathfrak{P}))^{\mathfrak{M}^{*}}\right)$. Now (8.23) implies that $\mathfrak{P} \triangleleft \mathfrak{M}^{*}$. Thus,

if $\mathfrak{P} \subseteq \mathfrak{M}^{*} \in \mathscr{L C S}(\mathfrak{S})$, then either $\mathfrak{P} \triangleleft \mathfrak{M}^{*}$ or $\mathfrak{M}^{*}$ satisfies Hypothesis 8.1 .

Let $\widetilde{\mathfrak{M}}$ be an element of $\mathscr{L S}(\mathfrak{S})$ which contains $N(\mathfrak{A} \cdot)$ and let $\mathfrak{P}_{0}=\boldsymbol{O}_{3}(\widetilde{\mathfrak{M}})$. We argue that

$$
\mathfrak{P} \rtimes N(\mathfrak{U} \cdot) \text {. }
$$

Namely, $\mathfrak{P} \subseteq N(\mathfrak{Q} \cdot)$. Also, $N(\mathfrak{H} \cdot)$ contains a $S_{3}$-subgroup of $\mathfrak{R}_{\dot{j}}$. By (8.27), this implies that $N(\mathfrak{2} \cdot)$ has more than one $S_{3}$-subgroup so (8.32) holds. By (8.31), it follows that $\left|\widetilde{\mathfrak{R}}_{0}\right|=3^{5}$ and that $\widetilde{\mathfrak{M}}=N(\mathfrak{X})$, where $\mathfrak{X}$ is some subgroup of $\boldsymbol{Z}(\mathfrak{P})$ of order 3 . Clearly, $\mathfrak{X} \neq \mathfrak{B}_{i}$, since $N(\mathfrak{H} \cdot) \nsubseteq$

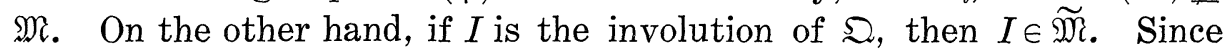
$\mathfrak{A} \cdot$ and $\mathfrak{B}_{i}$ are the only subgroups of $\boldsymbol{Z}(\mathfrak{P})$ of order 3 which are normalized by $I$, it follows that $\mathfrak{X}=\mathfrak{A} \cdot$.

Since $\mathfrak{M} \neq \widetilde{\mathfrak{M}}$, so also $\mathfrak{P}_{0} \neq \widetilde{\mathfrak{R}}_{0}$. Hence, (8.20) implies that $\mathfrak{P}_{0} \cap \tilde{\mathfrak{F}}_{0}$ is of order $3^{4}$. Now (8.24) implies that $\mathfrak{D} \subseteq \mathfrak{P}_{0} \cap \tilde{\mathfrak{P}}_{0}$, so by $(8.28)$, we have

$$
\mathfrak{D}=\mathfrak{P}_{0} \cap \tilde{\mathfrak{P}}_{0} \text {. }
$$

Since $\widetilde{\mathfrak{M}}=N(\mathfrak{A} \cdot)$, we have $\mathfrak{D} \cdot \subseteq O_{3}(\widetilde{\mathfrak{M}})$. Hence,

$$
\tilde{\mathfrak{P}}_{0}=\langle\mathfrak{D}, \mathfrak{D} \cdot\rangle \text {. }
$$

Since $I$ inverts $\mathfrak{P} \cdot$, we have $I \in \widetilde{\mathfrak{M}}$. Let $\widetilde{\mathfrak{Q}}$ be a $S_{2}$-subgroup of $O^{3 \prime}(\widetilde{\mathfrak{M}})$ which is normalized by $I$. Thus, $\tilde{\mathfrak{D}}$ is a quaternion group, and by (8.22) (with $\widetilde{\mathfrak{M}}$ in the role of $\mathfrak{M}$ ), we get that

$$
\widetilde{\mathfrak{\Omega}}\langle I\rangle \text { is a } S_{2} \text {-subgroup of } \widetilde{\mathfrak{M}} \text {. }
$$

Let $J$ be the involution of $\widetilde{\mathfrak{a}}$ and set

$$
\mathfrak{S}=\langle I, J\rangle \text {. }
$$


Thus, $\mathfrak{S}$ is a four-group and $\mathfrak{S} \subseteq N(\mathfrak{P})$. Since $\mathfrak{B}_{i}=Z(\mathfrak{P}) \cap C(I)$, it follows that $\mathfrak{S} \subseteq \mathfrak{M}$. Hence,

$$
\mathfrak{P} \triangleleft \mathfrak{P S}=\mathfrak{M} \cap \widetilde{\mathfrak{M}} \text {. }
$$

Notice that by (8.22), we have $\mathfrak{M}=\mathscr{S}\langle J\rangle$. Consider $C_{\mathfrak{M}}(I)=C_{\mathscr{S}}(I)\langle J\rangle$. By (8.5), it follows that $\mathfrak{C _ { \Im }}(I)$. Thus, $J$ normalizes $\mathfrak{\Omega}$ so that

$$
\mathfrak{Q}\langle J\rangle \text { is a } S_{2} \text {-subgroup of } \mathfrak{M} \text {. }
$$

Let $Q$ be an element of $\Omega$ of order 4 which normalizes $\mathfrak{S}$ and let $\widetilde{Q}$ be an element of $\tilde{\mathfrak{Q}}$ of order 4 which normalizes $\mathscr{H}$. By (8.22), it follows that $N_{\mathfrak{M}}(\mathfrak{Q}) / N_{\mathfrak{P}_{0}}(\mathfrak{Q}) \cong G L(2,3)$. Similarly for $\widetilde{\mathfrak{D}}$. Hence, neither $Q$ nor $\widetilde{Q}$ centralizes $\mathfrak{S}$, that is,

$$
\langle Q, \mathfrak{S}\rangle \text { and }\langle\widetilde{Q}, \mathfrak{S}\rangle \text { are dihedral groups of order } 8 \text {. }
$$

We set

$$
I_{1}=J Q, \quad I_{2}=I Q .
$$

Thus, $I_{1}$ and $I_{2}$ are involutions and

$$
\begin{array}{ll}
I_{1} J I_{1}=J I, & I_{1} I I_{1}=I, \\
I_{2} J I_{2}=J, & I_{3} I I_{2}=I J .
\end{array}
$$

Finally, we get

$$
\mathfrak{W}_{0}=\left\langle I_{1}, I_{2}\right\rangle \subseteq N(\mathfrak{S}) \text {. }
$$

We next show that $\mathfrak{P}_{0}$ is complemented in $\mathfrak{M}$. It is clear from the structure of $\mathfrak{M}=\mathfrak{R}_{i}$ that $C_{\mathfrak{M}}(I)$ covers $\mathfrak{N}_{i} / \mathbb{E}_{i}=\mathfrak{N}_{i} / \mathfrak{\Re}_{0}$ and that $C_{\mathfrak{M}}(I) \cap \mathfrak{P}_{0}=\mathfrak{B}_{0}$. Hence $\mathfrak{M}$ will split over $\mathfrak{P}_{0}$ if $C_{\mathfrak{M}}(I)$ splits over $\mathfrak{B}_{i}$. Since $\mathfrak{B}_{i}$ is an abelian 3-group, this occurs if and only if a $S_{3}$-subgroup of $C_{\mathfrak{M}}(I)$ splits over $\mathfrak{B}_{i}$, hence, if and only if $C_{\mathfrak{P}}(I)$ is elementary of order $3^{2}$. Regarding $I$ as an element of $\widetilde{\mathfrak{M}}$, we know from the structure of this group that $C_{\mathfrak{P}_{\mathfrak{\beta}}}(I)=\boldsymbol{C}_{\widetilde{\mathfrak{P}}_{0}}(I)$. But $\tilde{\mathfrak{P}}_{0}$ has exponent 3 , by $(8.16)$ and (8.19). Since the structure of $\mathfrak{M}$ implies that $\left|C_{\mathfrak{S}}(I)\right|=3^{2}$, we have proved that

$$
\mathfrak{M} \text { splits over } \mathfrak{P}_{0} ; \widetilde{\mathfrak{W}} \text { splits over } \tilde{\mathfrak{P}}_{0} \text {. }
$$

We define

$$
\mathfrak{X}_{6}=\mathfrak{A} \cdot \mathfrak{X}^{5}=\mathfrak{B}_{i}, \mathfrak{X}_{4}=\mathfrak{2} \cdot Q, \mathfrak{X}_{3}=\mathfrak{X}_{5}^{\check{Q}} .
$$

Since $\left\langle\mathfrak{X}_{4}, \mathfrak{X}_{5}, \mathfrak{X}_{6}\right\rangle=\mathfrak{B}^{\mathfrak{M}}$ and $\left\langle\mathfrak{X}_{3}, \mathfrak{X}_{5}, \mathfrak{X}_{6}\right\rangle=\mathfrak{B}^{\tilde{M}}$, (8.28) implies that

$$
\mathfrak{D}=\left\langle\mathfrak{X}_{3}, \mathfrak{X}_{4}, \mathfrak{X}_{5}, \mathfrak{X}_{6}\right\rangle \text {. }
$$

We set 


$$
\mathfrak{X}_{1}=\mathfrak{X}_{3}^{Q}, \mathfrak{X}_{2}=\mathfrak{X}_{4}^{\tilde{Q}}
$$

It then follows that

$$
\mathfrak{X}_{i+1} \ldots \mathfrak{X}_{6} \text { is a subgroup of } \mathfrak{P} \text { of }
$$

$$
\text { order } 3^{6-i}, i=0, \cdots, 5 \text {. }
$$

Further, by construction,

$$
\mathfrak{S} \text { normalizes } \mathfrak{X}_{i}, 1 \leqq i \leqq 6 \text {. }
$$

We now set up a 6 by 2 array whose $(i, j)$ entry is $\mathfrak{X}_{i}^{I} i$, in case $\mathfrak{X}_{i}^{I} \subseteq \mathfrak{B}$, and is - otherwise.

\begin{tabular}{c|c|c} 
& $I_{1}$ & $I_{2}$ \\
\hline $\mathfrak{X}_{1}$ & $\mathfrak{X}_{3}$ & - \\
$\mathfrak{X}_{2}$ & - & $\mathfrak{X}_{4}$ \\
$\mathfrak{X}_{3}$ & $\mathfrak{X}_{1}$ & $\mathfrak{X}_{5}$ \\
$\mathfrak{X}_{4}$ & $\mathfrak{X}_{6}$ & $\mathfrak{X}_{2}$ \\
$\mathfrak{X}_{5}$ & $\mathfrak{X}_{5}$ & $\mathfrak{X}_{3}$ \\
$\mathfrak{X}_{6}$ & $\mathfrak{X}_{4}$ & $\mathfrak{X}_{6}$
\end{tabular}.

We will eventually determine $\mathfrak{M}$ and $\widetilde{\mathfrak{M}}$ in terms of generators and relations. To do this, a number of choices must be made, and some care is required to guarantee that these choices are possible. We have already chosen the groups $\mathfrak{X}_{i}, 1 \leqq i \leqq 6$, each of order 3 and each normalized by $\mathfrak{S}$. Since $\mathfrak{X}_{1} \nsubseteq \tilde{\mathfrak{P}}_{0}$, and since $\mathfrak{X}_{1}$ centralizes $J$, we have $\mathfrak{X}_{1} \subseteq C_{\mathfrak{M}}(J)=\tilde{\mathfrak{Q}}\langle I\rangle \mathfrak{X}_{1} \mathfrak{X}_{6}$. Hence, $\mathfrak{X}_{1}$ normalizes $\tilde{\mathfrak{Q}}$. We therefore may choose a generator $X_{1}$ of $\mathfrak{X}_{1}$ such that $X_{1} \widetilde{Q}$ has order 3. Namely, let $X_{1}$ be any generator for $\mathfrak{X}_{1}$. Then $\left(X_{1} \widetilde{Q}\right)^{3} \in\langle J\rangle$, so either $\left(X_{1} \widetilde{Q}\right)^{3}=1$ or $\left(X_{1} \widetilde{Q}\right)^{3}=J$. Since $\widetilde{Q} J=\widetilde{Q}^{-1}$, in the second case we get $\left(X_{1} \widetilde{Q}^{-1}\right)^{3}=1$, or equivalently, $\left(\widetilde{Q} X_{1}^{-1}\right)^{3}=1$, or equivalently, $\left(X_{1}^{-1} \widetilde{Q}\right)^{3}=1$. Thus, we may assume that

$$
\left(X_{1} \widetilde{Q}\right)^{3}=1 .
$$

For the same reason, we may choose a generator $X_{2}$ for $\mathfrak{X}_{2}$ such that

$$
\left(X_{2} Q\right)^{3}=1 \text {. }
$$

We set $X_{3}=X_{1}^{Q}, X_{4}=X_{2}^{\widetilde{Q}}, X_{5}=X_{3}^{\widetilde{Q}}, X_{6}=X_{4}^{Q}$. Notice that

$$
\left\langle X_{i}\right\rangle=\mathfrak{X}_{i}, 1 \leqq i \leqq 6 \text {. }
$$

It is now convenient to draw up a table listing the action of $\mathfrak{F}$ on each $\mathfrak{X}_{i}$. This information is available since we know the action 
of $\mathfrak{F}$ on $\mathfrak{X}_{1}$ and $\mathfrak{X}_{2}$, and we know the action of $Q$, $\widetilde{Q}$ on $\mathfrak{S}$, and of course we know the way in which $Q, \widetilde{Q}$ permute the $\mathfrak{X}_{i}$. The result of this calculation is given in the following self-explanatory table:

\begin{tabular}{c|r|r} 
& \multicolumn{1}{|c|}{$I$} & \multicolumn{1}{|c}{$J$} \\
\hline$X_{1}$ & -1 & 1 \\
$X_{2}$ & 1 & -1 \\
$X_{3}$ & -1 & -1 \\
$X_{4}$ & -1 & -1 \\
$X_{5}$ & 1 & -1 \\
$X_{6}$ & -1 & 1
\end{tabular}

Since $Q^{2}=I, \widetilde{Q}^{2}=J$, we couple our two tables and determine the action of $Q, \widetilde{Q}$ on $\mathfrak{P}_{0}, \mathfrak{P}_{0}$ respectively. The result of this calculation is summarized below:

\begin{tabular}{|c|c|c|}
\hline & $Q$ & $\widetilde{Q}$ \\
\hline$X_{1}$ & $X_{3}$ & - \\
\hline$X_{2}$ & - & $X_{4}$ \\
\hline$X_{3}$ & $X_{1}^{-1}$ & $X_{5}$ \\
\hline$X_{4}$ & $X_{6}$ & $X_{2}^{-1}$ \\
\hline$X_{5}$ & $X_{5}$ & $X_{3}^{-1}$ \\
\hline$X_{6}$ & $X_{4}^{-1}$ & $X_{6}$ \\
\hline
\end{tabular}

It remains to determine the commutation relations in $\mathfrak{P}$. Since $\mathfrak{D}$ is abelian and $\mathfrak{D}_{i}=\boldsymbol{Z}\left(\mathfrak{S}_{0}\right)$, we get

$$
\begin{array}{ll}
{\left[X_{i}, X_{j}\right]=1,} & 3 \leqq i, j \leqq 6, \\
{\left[X_{1}, X_{j}\right]=1,} & 4 \leqq j \leqq 6 .
\end{array}
$$

Since $\left\langle\mathfrak{X}_{3}, \mathfrak{X}_{5}, \mathfrak{X}_{6}\right\rangle=\boldsymbol{Z}\left(\tilde{\mathfrak{R}}_{0}\right)$, we get

$$
\left[X_{2}, X_{3}\right]=\left[X_{2}, X_{5}\right]=\left[X_{2}, X_{6}\right]=1 .
$$

The three remaining commutation relations can be written as follows:

$$
\left[X_{1}, X_{3}\right]=X_{5}^{a} \text {, }
$$

$$
\left[X_{2}, X_{4}\right]=X_{6}^{b} \text {. }
$$$$
\left[X_{1}, X_{2}\right]=X_{3}^{c} X_{4}^{d} X_{5}^{e} X_{6}^{f} \text {. }
$$

Here $a, b, c, d, e, f \in F_{3}$. Since $\mathfrak{P}_{0}$ and $\tilde{\mathfrak{P}}_{0}$ are non abelian, we see that 
$a b \neq 0$. It follows from (8.29) that $\left[X_{1}, X_{2}, X_{2}\right]$ does not lie in $\mathfrak{X}_{5}$, so $d \neq 0$. By symmetry, $c \neq 0$. To determine the values $a$ through $f$ explicitly, we make use of the following identities:

$$
\begin{aligned}
{[A B, C] } & =[A, C][A, C, B][B, C] \\
{[A, B C] } & =[A, C][A, B][A, B, C] \\
{\left[A^{-1}, C\right] } & =\left[A, C, A^{-1}\right]^{-1}[A, C]^{-1} \\
{\left[A, B^{-1}\right] } & =\left[A, B, B^{-1}\right]^{-1}[A, B]^{-1} \\
{\left[A^{-1}, B^{-1}\right] } & =\left[A, B^{-1}, A^{-1}\right]^{-1}\left[A, B^{-1}\right]^{-1} .
\end{aligned}
$$

Since $X_{2} Q$ has order 3 , we have

$$
Q^{-1} X_{2} Q=I Q X_{2} Q=I X_{2}^{-1} Q^{-1} X_{2}^{-1}=X_{2}^{-1} Q X_{2}^{-1} \text {. }
$$

Using this relation, conjugate (8.59) by $Q$, to obtain

$$
\left[X_{3}, X_{2}^{-1} Q X_{2}^{-1}\right]=X_{1}^{-c} X_{6}^{d} X_{5}^{e} X_{4}^{-f} \text {. }
$$

Since $X_{2}$ and $X_{3}$ commute, we have

$$
\left[X_{3}, X_{2}^{-1} Q X_{2}^{-1}\right]=\left[X_{3}, Q X_{2}^{-1}\right] \text {. }
$$

By the preceding identities, $\left[X_{3}, Q X_{2}^{-1}\right]=\left[X_{3}, Q\right]\left[X_{3}, Q, X_{2}^{-1}\right]$. Now

$$
\left[X_{3}, Q\right]=X_{3}^{-1} Q^{-1} X_{3} Q=X_{3}^{-1} X_{1}^{-1},
$$

so that

$$
\left[X_{3}, Q X_{2}^{-1}\right]=X_{3}^{-1} X_{1}^{-1}\left[X_{3}^{-1} X_{1}^{-1}, X_{2}^{-1}\right] \text {. }
$$

Since $\mathfrak{X}_{2}$ and $\mathfrak{X}_{3}$ commute, we have $\left[X_{3}^{-1} X_{1}^{-1}, X_{2}^{-1}\right]=\left[X_{1}^{-1}, X_{2}^{-1}\right]$. Now by the preceding identities, we have

$$
\begin{aligned}
{\left[X_{1}^{-1}, X_{2}^{-1}\right]=} & {\left[X_{1}, X_{2}^{-1}, X_{1}^{-1}\right]^{-1}\left[X_{1}, X_{2}^{-1}\right]^{-1} } \\
= & {\left[\left[X_{1}, X_{2}, X_{2}^{-1}\right]^{-1}\left[X_{1}, X_{2}\right]^{-1}, X_{1}^{-1}\right]^{-1} } \\
& \times\left(\left[X_{1}, X_{2}, X_{2}^{-1}\right]^{-1}\left[X_{1}, X_{2}\right]^{-1}\right)^{-1} .
\end{aligned}
$$

Since $\left[X_{1}, X_{2}, X_{2}^{-1}\right] \in \mathfrak{F}_{i}$, it follows that

$$
\left[\left[X_{1}, X_{2}, X_{2}^{-1}\right]^{-1}\left[X_{1}, X_{2}\right]^{-1}, X_{1}^{-1}\right]^{-1}=\left[\left[X_{1}, X_{2}\right]^{-1}, X_{1}^{-1}\right]^{-1} \text {. }
$$

We get that $\left[X_{1}^{-1}, X_{2}^{-1}\right]=X_{5}^{a c} X_{3}^{c} X_{4}^{d} X_{5}^{e} X_{6}^{f+b d}$. Since $X_{3}^{-1} X_{1}^{-1}=X_{1}^{-1} X_{3}^{-1} X_{5}^{-a}$, we see that $\left[X_{3}, X_{2}^{-1} Q X_{2}^{-1}\right]=X_{1}^{-1} X_{3}^{-1} X_{5}^{-a+a c} X_{3}^{c} X_{4}^{d} X_{5}^{e} X_{6}^{f+b d}$. This gives us the following equations: $c=1, d=-f, f+b d=d$. Conjugating (8.59) successively by $I, J, I J$ and using the fact that $d \neq 0$ yield the values $b=-1, a=e, d=-f$. No more information is forthcoming from $\mathfrak{M}$, so we conjugate $(8.59)$ by $\widetilde{Q}$ and work in $\widetilde{\mathfrak{M}}$. We state the result of these calculations: 


$$
a=-1, b=-1, c=1, d=-1, e=-1, f=1 .
$$

Let $\mathfrak{R}^{*}=\left\langle\mathfrak{X}_{2}, \mathfrak{X}_{5}\right\rangle$ and note that $\mathfrak{R}^{*}=C_{\mathfrak{P}}(I)$. By construction, $\mathfrak{X}_{2} \subseteq \boldsymbol{O}_{3}(\widetilde{\mathfrak{M}})$ and $\mathfrak{X}_{3} \subseteq \boldsymbol{Z}\left(\boldsymbol{O}_{3}(\widetilde{\mathfrak{M}})\right)$. Hence,

$$
\boldsymbol{C}_{\mathfrak{P}}\left(\mathfrak{X}_{2}\right)=\boldsymbol{C}_{\mathfrak{P}}\left(\Re^{*}\right)=\left\langle X_{2}, X_{3}, X_{5}, X_{6}\right\rangle,
$$

so that $\left|\mathfrak{P}: \boldsymbol{C}_{\mathfrak{P}}\left(\mathfrak{R}^{*}\right)\right|=9$. With $\mathfrak{R}^{*}$ in the role of $\mathfrak{A}$ in Lemma 7.6 (c), it follows that $\Re^{*}$ centralizes every abelian subgroup of $И\left(\Re^{*} ; 2\right)$.

Since $\boldsymbol{O}^{3^{\prime}}(\mathfrak{M}) \cap C(I)=\mathfrak{R}^{*} \mathfrak{Q}$, it follows that $\mathfrak{Q}$ is normalized by $\mathfrak{R}^{*}$ but is not centralized by $\Re^{*}$. Let $\mathfrak{I}^{*}$ be a $S_{2,3}$-subgroup of $C(I)$ which contains $\mathfrak{R}^{*} \mathfrak{O}$. Then $\mathfrak{I}^{*}$ contains an element $\mathscr{U}(2)$. Let $\mathfrak{I}_{2}$ be a $S_{2^{-}}$ subgroup of $\mathfrak{I}^{*}$ which contains $\Omega$. By Lemma 7.5, there is an element $\mathfrak{M}_{1}$ of $\mathscr{L C S}(\mathfrak{S})$ such that $\left(\mathfrak{R}^{*}, \mathfrak{I}^{*}, \mathfrak{I}_{2}, \Re=\boldsymbol{O}_{2}\left(\mathfrak{M}_{1}\right), \mathfrak{M}_{1}\right)$ satisfies all parts of Lemma 7.5 with $\mathfrak{R}^{*}$ in the role of $\mathfrak{B}, \mathscr{R}$ in the role of $\mathfrak{S}_{2}, \mathfrak{M}_{1}$ in the role of $\mathfrak{M}$. Since by (e) of Lemma 7.5, $\cong \subseteq \Re$, it follows that $\mathfrak{M}_{1}=C(I)$. Hence, $J \in \mathfrak{M}_{1}$.

The next task is shown that

$$
N(\mathfrak{D}) \subseteq N(\mathfrak{P}) \text {. }
$$

By our preceding results, $\mathfrak{D} \triangleleft \mathfrak{P}$. It is straightforward to verify that

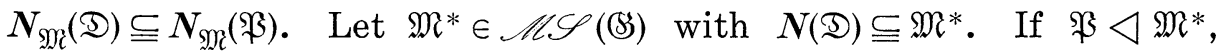
we have our desired containment. Otherwise, $\mathfrak{M}^{*}$ satisfies Hypothesis 8.1. Hence, $N(\mathfrak{D})=N_{\mathfrak{M}^{*}}(\mathfrak{D}) \subseteq N_{\mathfrak{M}^{*}}(\mathfrak{P}) \subseteq N(\mathfrak{P})$, as desired.

We next show that

$$
\text { if } X \in \mathfrak{X}_{2}^{\sharp}, Y \in \mathfrak{X}_{3}^{\sharp} \text {, then }|C(X Y)|_{3}=3^{4} \text {. }
$$

Let $Z=X Y$. Let $X^{*}=X^{\tilde{Q}}, Y^{*}=Y^{\tilde{Q}}, Z^{*}=X^{*} Y^{*}$. Then $X^{*} \in \mathfrak{X}_{4}$, $Y^{*} \in \mathfrak{X}_{3}$, so it suffices to show that $\mathcal{D}$ is a $S_{3}$-subgroup of $C\left(Z^{*}\right)$. Suppose false. Let $\widetilde{\mathfrak{D}}$ be a $S_{3}$-subgroup of $C\left(\mathfrak{X}^{*}\right)$ which contains $\mathfrak{D}$, and let $\mathfrak{D} \leqq \mathfrak{D}^{*} \subseteq \widetilde{D}$, with $\left|\mathfrak{D}^{*}: \mathfrak{D}\right|=3$. Then $\mathfrak{D}^{*} \subseteq N(\mathfrak{D}) \subseteq N(\mathfrak{P})$, so $\mathfrak{D}^{*} \subseteq \mathfrak{B}$. However, $\mathfrak{D}=C_{\mathfrak{F}}\left(Z^{*}\right)$. Notice that we have shown that D $\triangle \boldsymbol{C}\left(Z^{*}\right)$. Namely, $\mathfrak{D}$ is a $S_{3}$-subgroup of $\boldsymbol{C}\left(Z^{*}\right)$, and since $\mathfrak{D} \in \mathscr{S}_{\text {s }_{3}}(P)$, we have $\boldsymbol{O}_{3^{\prime}}\left(\boldsymbol{C}\left(Z^{*}\right)\right)=1$ so that

(D) is a normal $S_{3}$-subgroup of $C\left(Z^{*}\right)$.

Retaining the preceding notation we will show that $\langle I\rangle=\boldsymbol{C}_{\mathscr{R}}(Z)$. Suppose false. Since $\langle I\rangle=C_{\Re^{\prime}}\left(\Re^{*}\right)$, it follows that $1 \neq\left[C_{\Re^{\prime}}(Z), \Re^{*}\right]$. This violates the fact that $C(Z)$ is 3 -closed by (8.63).

We next observe that $\mathfrak{X}_{2} \sim \mathfrak{X}_{6}$ so that $\left|C\left(\mathfrak{X}_{2}\right)\right|_{2}=\left|C\left(\mathfrak{X}_{5}\right)\right|_{2}=8$. These equalities together with the preceding paragraph show that $\Re$ is extra special of width 2 and that

$\Re$ is the central product of quaternion groups

$$
\mathfrak{Q}, \mathfrak{\Omega}_{1} \text {, where } \quad \mathfrak{\Omega}=\boldsymbol{C}_{\Re}\left(\mathfrak{X}_{5}\right), \mathfrak{\Omega}_{1}=\boldsymbol{C}_{\mathfrak{\Re}}\left(\mathfrak{X}_{2}\right) \text {. }
$$


This choice of notation conforms with our previous definition of $\mathfrak{}$.

Since $\mathfrak{R}^{*}$ maps onto a $S_{3}$-subgroup of $\mathfrak{A}_{\mathscr{S}}(\Re)$, it follows that $\mathfrak{R} \Re \triangleleft \mathfrak{M}_{1}$. Let

$$
\stackrel{\Omega}{ }=N_{\mathfrak{M}_{1}}\left(\Re^{*}\right),
$$

so that $\mathfrak{Q} \cap \Re=\langle I\rangle$, and $\mathfrak{M}_{1}=\Re \mathscr{R}$. $\mathfrak{R}$ acts as a permutation group on the subgroups of $\Re^{*}$ of order 3 . By the previous arguments, $\mathfrak{X}_{2}$ and $\mathfrak{X}_{5}$ are permuted among themselves. Let

$$
\mathfrak{2}^{*}=\boldsymbol{N}_{\mathfrak{\Omega}}\left(\mathfrak{X}_{2}\right)=\boldsymbol{N}_{\mathfrak{\Omega}}\left(\mathfrak{X}_{5}\right)
$$

so that $\left|\mathfrak{R}: \mathbb{R}^{*}\right| \leqq 2$. Also, if $L \in \mathbb{R}^{*}$ and $L$ centralizes $\mathfrak{X}_{5}$, then $L \in \Re^{*}\langle I\rangle$. Hence, $\mathbb{2}^{*}=\mathfrak{R}^{*} \mathfrak{K}$, and $\left|\mathfrak{M}_{1}: \mathfrak{R}^{*}\right| \leqq 2$. Let $\mathfrak{\Omega}_{2}$ be a $S_{2}$-subgroup of $\mathbb{R}$ which contains $\mathfrak{S}$. Thus, $\left|\mathfrak{L}_{2}\right|=4$ or 8 .

We must now show that

$$
\mathfrak{L}=\mathbb{2}^{*} \text {. }
$$

Suppose false. Since $\Re^{* \widetilde{\mathscr{Q}}}=\left\langle\mathfrak{X}_{3}, \mathfrak{X}_{4}\right\rangle$, it follows that $\mathbb{2}^{\widetilde{\mathscr{Q}}}$ normalizes $\left\langle\mathfrak{X}_{3}, \mathfrak{X}_{4}\right\rangle$. From (8.63), we conclude that $\mathfrak{D}$ char $C\left(\mathfrak{X}_{3} \mathfrak{X}_{4}\right)$. Hence, $N\left(\mathfrak{X}_{3} \mathfrak{X}_{4}\right) \subseteq$ $N(\mathfrak{D})$. Now by $(8.61)$, we have $N(\mathfrak{D}) \subseteq N(\mathfrak{P})$. Thus $2^{\tilde{Q}}$ normalizes $\mathfrak{P}$.

It is a straightforward consequence of (8.55) through (8.60) that $\mathfrak{P}_{0} \cup \widetilde{\mathfrak{P}}_{0}$ is the set of elements of $\mathfrak{P}$ of order at most 3 . Hence, $\mathfrak{P}$ contains exactly $3^{6}-2 \cdot 3^{5}+3^{4}=4.3^{4}$ elements of order 9 . Thus, some involution $I_{0}$ of $\mathcal{R}_{2}^{\tilde{Q}}$ centralizes an element $P$ of $\mathfrak{P}$ of order 9 . It is clear from (8.53) that $I_{0} \notin \mathfrak{S}$.

If $X \in \mathfrak{X}_{5}^{\sharp} \mathfrak{X}$, we will show that $C(X) \subseteq N(\mathfrak{P})$. Suppose false. Let $\mathfrak{M}^{*} \in \mathscr{M C S}^{(\mathbb{S})}$ with $C(X) \subseteq \mathfrak{M}^{*}$. We may apply all the preceding results to $\mathfrak{M}^{*}$ in place of $\mathfrak{M}$ and conclude that $\boldsymbol{O}_{3}\left(\mathfrak{M}^{*}\right)$ is of exponent 3 and order $3^{5}$. However, $\mathfrak{B}_{0}$ and $\tilde{\mathfrak{P}}_{0}$ are the only subgroups of $\mathfrak{P}$ meeting these conditions, so $\boldsymbol{C}(X) \subseteq \mathfrak{M}$ or $\boldsymbol{C}(X) \subseteq \widetilde{\mathfrak{M}}$, from which the desired containment is obvious. In particular,

$$
C\left(P^{3}\right) \subseteq N(\mathfrak{P}) \text {. }
$$

Let $\Re_{2}$ be a $S_{2}$-subgroup of $N(\mathfrak{P})$ which contains $\mathbb{2}_{2}^{\widetilde{Q}}$. By (8.23) $\mathfrak{N}_{2}$ is faithfully represented on $\mathfrak{B}=\boldsymbol{Z}(\mathfrak{P})=\left\langle\mathfrak{X}_{5}, \mathfrak{X}_{6}\right\rangle$. It is clear that Aut $(\mathfrak{P})$ is a 2,3 -group, so we conclude that

$$
N(\mathfrak{P})=\mathfrak{B} \mathbb{Q}^{\tilde{Q}} \text {. }
$$

It now follows from (8.68), (8.69), and (8.53) that

$$
C\left(P^{3}\right)=\mathfrak{P}\left\langle I_{0}\right\rangle \text {. }
$$

By hypothesis, $\boldsymbol{C}\left(I_{0}\right)$ is solvable. Let $\mathfrak{F}=\boldsymbol{O}_{2}\left(\boldsymbol{C}\left(I_{0}\right)\right)$. Suppose $\langle P\rangle$ acts faithfully on $\mathfrak{F}$. Then $m(\mathfrak{F}) \geqq 6$, since $P$ has order 9 . But $\mathfrak{M}_{1}$ 
contains a $S_{2}$-subgroup of $\mathfrak{B S}$, and since $S_{2}$-subgroups of $\mathfrak{M}_{1}$ are extensions of $\Omega$ by a 4 group, it follows that every 2-subgroup of (B) is generated by 4-elements (naturally, this uses the action of the 4-group on $\Re)$. Hence, $P^{3}$ centralizes $\mathfrak{F}$. By (8.70), we get $\mathfrak{F}=\left\langle I_{0}\right\rangle$.

By Lemma 5.38 (a)(ii), $C\left(I_{0}\right)$ contains an element $\mathfrak{U}$ of $\mathscr{\mathscr { C }}(2)$. Since $\boldsymbol{O}_{2}\left(\boldsymbol{C}\left(I_{0}\right)\right)=\left\langle I_{0}\right\rangle$, we get that $\boldsymbol{O}_{2,2^{\prime}}\left(\boldsymbol{C}\left(I_{0}\right)\right)=\left\langle I_{0}\right\rangle \times \boldsymbol{O}_{2^{\prime}}\left(\boldsymbol{C}\left(I_{0}\right)\right)$, so that by Lemma 7.1, $\mathfrak{U}$ centralizes $\boldsymbol{O}_{2,2^{\prime}}\left(\boldsymbol{C}\left(I_{0}\right)\right)$. But $\boldsymbol{C}\left(I_{0}\right)$ is solvable, so that $\boldsymbol{O}_{2,2^{\prime}}\left(\boldsymbol{C}\left(I_{0}\right)\right)$ contains its centralizer. Thus, $\mathfrak{u} \subseteq \boldsymbol{O}_{2,2^{\prime}}\left(C\left(I_{0}\right)\right)$, an absurdity. This contradiction establishes (8.67). Notice that (8.67) is equivalent to

$$
\mathfrak{M}_{1}=\mathfrak{R}^{*}\langle J\rangle \text {. }
$$

Since $J$ inverts $\Re^{*}$, it follows that $\mathfrak{Q}\langle J\rangle$ and $\mathfrak{\Omega}_{1}\langle J\rangle$ are both isomorphic to $S_{2}$-subgroups of $G L(2,3)$. This implies that

$$
C_{\Re_{1}}(J) \text { is elementary of order } 8 \text {. }
$$

The hard work is now completed. We may now determine the Weyl group. Recall that $I_{1}=J Q, I_{2}=I \widetilde{Q}$, so that $I_{1}$ and $I_{2}$ are involutions. Let $W=I_{1} I_{2}$. Thus $W^{3}$ centralizes $\mathfrak{S}_{2}$. Since $W$ centralizes no element of $\mathfrak{S}^{\sharp}, W^{3}$ is not in $\mathfrak{S}^{\sharp}$. Since $W^{3} \in \boldsymbol{O}\left(\mathfrak{S}_{\mathfrak{C}}\right) \subseteq \mathfrak{M}_{1}$, and since the structure of $C_{\mathfrak{M}_{1}}(J)$ is given in (8.72), it follows that $W^{6}=1$, so that $W$ is of order 3 or 6 .

From (8.49), we get that $\mathfrak{X}_{1}^{W^{3}}=\mathfrak{X}_{1}^{I_{2}} \neq \mathfrak{X}_{1}$, and conclude that $W$ is of order 6 . Thus,

(8.73) $W_{0}=W^{3}$ is an involution in the center of $\left\langle I_{1}, I_{2}\right\rangle=\mathfrak{W}_{0}$.

We argue that

$$
\mathfrak{P} \cap \mathfrak{P}^{W_{0}}=1 .
$$

Since $\mathfrak{P} \cap \mathfrak{P}^{W_{0}}$ is normalized by $\mathfrak{S}$ and by $W_{0}$, (8.53) implies that if $\mathfrak{P}^{*}=\mathfrak{P} \cap \mathfrak{P}^{W_{0}}$, then

$$
\mathfrak{P}^{*}=\left(\mathfrak{P}^{*} \cap\left\langle\mathfrak{X}_{1}, \mathfrak{X}_{6}\right\rangle\right)\left(\mathfrak{P}^{*} \cap\left\langle\mathfrak{X}_{2}, \mathfrak{X}_{5}\right\rangle\right)\left(\mathfrak{P}^{*} \cap\left\langle\mathfrak{X}_{3}, \mathfrak{X}_{4}\right\rangle\right) .
$$

If $X \in \mathfrak{X}_{3}^{\sharp} \mathfrak{X}_{4}^{\sharp}$, we know that $C(X) \subseteq N(\mathfrak{D})$. This fact, coupled with (8.49) implies that $\mathfrak{P}^{*}=1$, so that $(8.74)$ holds.

Let $\mathfrak{B}=\mathfrak{B} \mathcal{C}_{\mathcal{C}} . \quad$ (No confusion with previous notation is to be feared.) We then get that $\mathfrak{M}=\mathfrak{B} \cup \mathfrak{B} I_{1} \mathfrak{B}, \widetilde{\mathfrak{M}}=\mathfrak{B} \cup \mathfrak{B} I_{2} \mathfrak{B}$. Hence, (8.49) implies that conditions (i') and (iv) of Théorème 1 of [40] are satisfied. Hence, $\mathfrak{B W}_{0} \mathfrak{B}=\mathfrak{B S}_{0}$ is a group and if we let $\mathfrak{B}_{X}$ be the largest subset of $\mathfrak{B}$ such that $\mathfrak{B}_{X}^{X} \subseteq \mathfrak{B}^{W_{0}}$, it follows easily from (8.74) that each element of $\mathbb{B}_{0}$ has a unique representation of the shape $B X B_{X}, B \in \mathfrak{B}, X \in \mathfrak{W}_{0}$, $B_{X} \in \mathfrak{B}_{X}$. Thus, $\left|\mathscr{S}_{0}\right|=\left|E_{2}(3)\right|$, by an easy calculation. Hence, (8.41), (8.50), (8.51), (8.53), (8.54), (8.57), (8.58), (8.59), (8.60), (8.73) determine the multiplication table of $\mathbb{S}_{0}$. Thus, if $\mathbb{S}^{*}$ is any group which satisfies 
the hypothesis of Theorem 8.1 and also satisfies Hypothesis 8.1, it follows that $\mathbb{S}^{*} *$ contains a subgroup isomorphic to $\mathbb{S}_{0}$. Since we may take $\mathbb{S}^{*}=E_{2}(3)$, it follows that $\mathbb{S}_{0} \cong E_{2}(3)$, and so $i\left(\mathbb{S}_{0}\right)=1$. Clearly, $\mathbb{S}_{0}$ contains $\mathfrak{M}_{1}$, so that $\mathscr{S}_{0}$ contains the centralizer of each of its involutions. Hence, $i(\mathbb{S})=1$, by Lemma 5.35.

Since $E_{2}(3)$ does not satisfy $E_{7,13}$ (by Sylow's theorem), it follows from Lemma 5.35 that $\mathbb{B S}_{0}=$ (S) $\cong E_{2}(3)$.

The remaining lemmas are proved under the following hypothesis:

Hypothesis 8.2. Whenever $\mathfrak{P} \subseteq \mathfrak{M} \in \mathscr{C l S}(\mathfrak{S})$ and $\mathfrak{B}=\Omega_{1}(\boldsymbol{Z}(\mathfrak{P}))^{\mathfrak{M}}$, then $V\left(\operatorname{ccl}_{\mathfrak{S}}(\mathfrak{D}) ; \mathfrak{B}\right) \subseteq C_{\mathfrak{M}}(\mathfrak{B})$.

We must derive a contradiction from this hypothesis. When this is done, the proof of Theorem 8.1 will be complete.

LEMMA 8.7. If $\mathfrak{I}$ is a 2, 3-subgroup of (S) and $\mathfrak{I}_{3}$ is a $S_{3}$-subgroup of $\mathfrak{T}$, then $V\left(\operatorname{ccl}_{\mathfrak{G}}(\mathfrak{D}) ; \mathfrak{I}_{3}\right) \triangleleft \mathfrak{I}$.

Proof. We assume without loss of generality that $\mathfrak{I}_{3} \subseteq \mathfrak{P}$. First, suppose $\mathfrak{I}_{3}=\mathfrak{P}$. Let $\mathfrak{I} \subseteq \mathfrak{M} \in \mathscr{M S}(\mathfrak{S})$, and let $\mathfrak{I}^{*}$ be a $S_{2,3}$-subgroup of $\mathfrak{M}$ containing $\mathfrak{T}$. Let $\mathfrak{B}=\Omega_{1}(\boldsymbol{Z}(\mathfrak{P}))^{\mathfrak{M}}$, $\mathfrak{\mathbb { N }}=\boldsymbol{C}_{\mathfrak{M}}(\mathfrak{B})$. As $\mathbb{\mathfrak { S }} \triangleleft \mathfrak{M}$, $\mathfrak{C} \cap \mathfrak{T}^{*}$ is a $S_{2,3}$-subgroup of $\mathfrak{c}$. By Hypothesis $8.2, \mathfrak{B}^{*} \subseteq \mathfrak{C} \cap \mathfrak{I}^{*}$, where $\mathfrak{B}^{*}=$ $\mathfrak{B}\left(\operatorname{ccl}_{\mathfrak{S}}(\mathfrak{D})\right.$; $\left.\mathfrak{P}\right)$. Since $\mathfrak{B} \subseteq \mathfrak{B}$, Lemmas 7.4 and 5.38 imply that $\left|\mathfrak{S} \cap \mathfrak{I}^{*}\right|$ is odd. Hence, $\mathfrak{S} \cap \mathfrak{I}^{*} \triangleleft \mathfrak{I}^{*}$ implies $\mathfrak{B}^{*}=V\left(\operatorname{ccl}_{\mathfrak{S}}(\mathfrak{D}) ; \mathfrak{S} \cap \mathfrak{I}^{*}\right) \triangleleft \mathfrak{I}^{*}$.

We may now assume that $\mathfrak{I}_{3} \subset \mathfrak{P}$. We proceed by induction on $|\mathfrak{P}| /\left|\mathfrak{I}_{3}\right|$. Let $\mathfrak{B}^{*}=\boldsymbol{V}\left(\operatorname{ccl} l_{(\mathfrak{S}}(\mathfrak{D}) ; \mathfrak{I}_{3}\right)$. As $\mathfrak{B}^{*}$ is generated by conjugates of $\mathfrak{B}$, it follows that $\mathfrak{B}^{*}$ centralizes $\boldsymbol{O}_{2}(\mathfrak{T})$. Hence, if $\mathfrak{B}^{*} \neq 1$, then $\boldsymbol{O}_{2}(\mathfrak{I})=1$, so that $\boldsymbol{O}_{2,3}(\mathfrak{I})=\boldsymbol{O}_{3}(\mathfrak{I})$. If $\mathfrak{B}^{*}=1$, the lemma is trivial, so suppose $\mathfrak{B}^{*} \neq 1$. In particular, $\boldsymbol{O}_{3}(\mathfrak{T}) \neq 1$. If $\mathfrak{I}_{3}$ is not a $S_{3}$-subgroup of $N\left(O_{3}(\mathfrak{T})\right.$ ), let $\mathfrak{T}^{*}$ be a $S_{2,3}$-subgroup of $N\left(O_{3}(\mathfrak{T})\right)$ containing $\mathfrak{I}$, and let $\mathfrak{I}_{3}^{*}$ be a $S_{3}$-subgroup of $\mathfrak{T}^{*}$ which contains $\mathfrak{I}_{3}$. Then $V\left(\operatorname{ccl} l_{\mathfrak{S}}(\mathfrak{D}) ; \mathfrak{I}_{3}^{*}\right) \triangleleft$

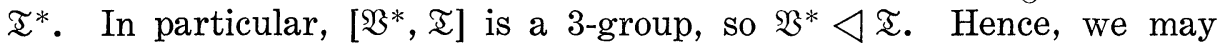
assume that $\mathfrak{I}_{3}$ is a $S_{3}$-subgroup of $N\left(O_{3}(\mathfrak{I})\right)$.

Let $\mathfrak{W}_{0}=\Omega_{1}\left(\boldsymbol{Z}\left(\boldsymbol{O}_{3}(\mathfrak{I})\right)\right)$, so that $\mathfrak{B} \subseteq \mathfrak{W}_{0}$. Since $\left|\boldsymbol{C}_{T}\left(\mathfrak{W}_{0}\right)\right|$ is odd, it follows that $\boldsymbol{O}_{3}(\mathfrak{I})=\boldsymbol{C}_{\mathfrak{I}}\left(\mathfrak{W}_{0}\right)$. Suppose $\mathfrak{B}^{*} \nsubseteq \boldsymbol{O}_{2}(\mathfrak{T})$. Choose $G$ in (S) so that $\mathfrak{D}^{a} \subseteq \mathfrak{S}^{*}$ but $\mathfrak{D}^{a} \nsubseteq \boldsymbol{O}_{3}(\mathfrak{I})$, and for any subset $\mathfrak{S}$ of $\mathbb{S}$, let $\mathfrak{S} \cdot=\mathfrak{S}^{a}$.

It is a straightforward consequence of Hypothesis 8.2 that $\mathfrak{D}^{\prime}=1$.

As $\mathfrak{D} \cdot$ acts nontrivially on $\boldsymbol{Q}_{3}^{1}(\mathfrak{T})$, we let $\mathfrak{Q}$ be a $\mathfrak{D} \cdot$-invariant subgroup of $\boldsymbol{Q}_{3}^{1}(\mathfrak{T})$ minimal subject to $[\mathfrak{D} \cdot \mathfrak{Q}] \neq 1$. Let $\mathfrak{D}_{0}^{*}=\boldsymbol{C}_{\mathfrak{D}} \cdot(\mathfrak{D})$, so that $\left|\mathfrak{D} \cdot: \mathfrak{D}_{0}\right|=3$. Thus, $\widetilde{\mathfrak{W}}_{0}=C_{\mathfrak{M}_{0}}\left(\mathfrak{D}_{0}^{*}\right)$ is invariant under $\mathfrak{D} \cdot$ and $\cong$.

Let $N=\left\{i \mid 1 \leqq i \leqq 4, \mathfrak{B}_{i} \subseteq \mathfrak{D}_{0}, \mathfrak{D}_{i}^{*} \nsubseteq \mathfrak{D}_{0}^{*}\right\}$. If $\mathfrak{B} \cdot \subseteq \mathfrak{D}_{0}$, then it is obvious that $N \neq \varnothing$. If $\mathfrak{B}^{\cdot} \subseteq \mathfrak{D}_{0}^{*}$, then no $\mathfrak{D}_{i}$ is contained in $\mathfrak{D}_{0}^{*}, 1 \leqq$ $i \leqq 4$. Since $\mathfrak{B} \cdot \cap \mathfrak{D}_{0}^{*}$ is of order 3 in this case, we again conclude that $N \neq \varnothing$. Choose $i \in N$. Thus, $\mathfrak{D}^{\cdot}=\left\langle\mathfrak{D}_{0}^{*}, \mathfrak{D}_{i}\right\rangle$ and $\left|\mathfrak{D}_{i}: \mathfrak{D}_{i} \cap \mathfrak{D}_{j}\right|=$ 
3. Since $\mathfrak{Q}$ is faithfully represented on $\widetilde{\mathfrak{W}}_{0}$, it follows that $\left[\mathfrak{D}_{i}^{*}, \widetilde{\mathfrak{W}}_{0}\right] \neq 1$. By Lemma 8.5, [ㅁ, $\left.\widetilde{\mathfrak{W}}_{0}\right]$ is of order 9 and is not centralized by $\mathfrak{D} \cdot$. Since $\mathfrak{B} \subseteq \mathfrak{M}_{0}$, so also $\mathfrak{B} \subseteq \widetilde{\mathfrak{B}}_{0}$. Hence, $\mathfrak{Q}$ centralizes some $B$ of $\mathfrak{B}^{\sharp}$, so if $\mathfrak{\Omega}=\mathfrak{Q}^{*} / C_{\mathfrak{T}}\left(\mathfrak{W}_{0}\right)$, then $\left\langle\mathfrak{D} \cdot \mathfrak{\Omega}^{*}\right\rangle \subseteq C(B)$. By the preceding argument, [*, $\left.\mathfrak{D}^{\cdot}\right]$ is a 3-group, violating the nontrivial action of $\mathfrak{D}^{\cdot}$ on D. Thus, $\mathfrak{B}^{*} \subseteq \boldsymbol{O}_{3}(\mathfrak{I})$, and so $\mathfrak{B}^{*} \triangleleft \mathfrak{I}$, completing the proof of this lemma.

For the remainder of this section, we let

$$
\mathfrak{B}=V\left(\operatorname{ccl}_{\mathscr{S}}(\mathfrak{D}) ; \mathfrak{P}\right), \mathfrak{R}=N(\mathfrak{B}) \text {. }
$$

Lemma 8.8. ( i ) $\mathfrak{i}$ contains no element of $\mathscr{T}(2)$. (See Definition 2.9.)

(ii) If $\mathfrak{I}_{0}$ is any 2-subgroup of $\mathfrak{R}$, then $\boldsymbol{A}_{\mathfrak{N}}\left(\mathfrak{I}_{0}\right)$ does not contain a subgroup of type $(3,3)$.

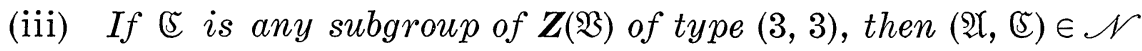
for all $\mathfrak{A} \in \mathscr{C}(2)$. (See Definition 7.2).

(iv) If $\mathfrak{I}_{1}$ is an abelian 2-subgroup of $\mathfrak{R}$, the $\boldsymbol{A}_{\mathfrak{R}}\left(\mathfrak{I}_{1}\right)$ is a $3^{\prime}$-group.

Proof. We first prove (iii). We invoke Lemma 7.4, so that (iii) will hold if we can show that $\sqrt{ }$ centralizes every element of $U(\mathfrak{S} ; 2)$. Suppose $\mathfrak{Q} \in \boldsymbol{U}(\mathfrak{S} ; 2)$ is minimal subject to $[\Omega, \mathfrak{E}] \neq 1$. Let $\mathfrak{E}_{0}=\boldsymbol{C}_{\mathbb{E}}(\mathfrak{Q}) \neq$ 1. Let $\mathfrak{I}$ be a $S_{2,3}$-subgroup of $\boldsymbol{C}\left(\mathfrak{S}_{0}\right)$ containing $\mathfrak{C} \cong$. Since $\boldsymbol{C}\left(\mathfrak{S}_{0}\right) \supseteqq \mathfrak{B}$, it follows that if $\mathfrak{I}_{0}$ is a $S_{2,3}$-subgroup of $C\left(\mathbb{E}_{0}\right)$ containing $\mathfrak{B}$, then $\mathfrak{B} \subseteq \boldsymbol{O}_{3}\left(\mathfrak{I}_{0}\right)$. By Lemma 0.7.5, we have $\mathfrak{S} \subseteq \boldsymbol{O}_{3}(\mathfrak{I})$, so that $[\mathfrak{N}, \mathfrak{E}] \subseteq$ $\mathfrak{O} \cap O_{3}(\mathfrak{T})=1$. (iii) is proved.

Let $\mathfrak{I} \in \mathscr{T}(2), \mathfrak{I} \subseteq \mathfrak{R}$. We may assume that $\mathfrak{I}$ is a noncyclic abelian group of order 8 . Since $\mathfrak{B} \subseteq \boldsymbol{Z}(\mathfrak{B}), \boldsymbol{Z}(\mathfrak{B})$ is noncyclic. Hence, $\mathfrak{I}$ contains an involution $I$ such that $C(I) \cap Z(\mathfrak{B})$ is noncyclic. Thus, $C(I)$ contains an element of $\mathscr{U}(2)$ and also a subgroup $\sqrt{5}$ of $Z(\mathfrak{B})$ of type $(3,3)$. By hypothesis, $C(I)$ is solvable, in violation of (iii). is proved.

(ii) is a straightforward consequence of (i).

To prove (iv), let $\mathfrak{I}_{1}$ be an abelian 2-subgroup of $\mathfrak{R}$ minimal subject to $3 \| \boldsymbol{A}_{\mathfrak{N}}\left(\mathfrak{I}_{1}\right) \mid$. Thus, $\mathfrak{I}_{1}$ is a four-group, and the involutions of $\mathfrak{I}_{1}$ are all $\mathfrak{N}$-conjugate. Thus, (iii) implies that $C(I) \cap Z(\mathfrak{B})$ is cyclic for all $I \in \mathfrak{I}_{1}^{\sharp}$. This implies that $\left|\Omega_{1}(\boldsymbol{Z}(\mathfrak{B}))\right| \leqq 3^{3}$. Since the reverse inequality holds by $(B)$, we find that $\boldsymbol{Z}(\mathfrak{P}) \cap \boldsymbol{Z}(\mathfrak{B})$ is cyclic. This is not the case, since $\mathfrak{B} \subseteq \boldsymbol{Z}(\mathfrak{P}) \cap \boldsymbol{Z}(\mathfrak{B})$. (iv) is proved.

Lemma 8.9. If $\mathfrak{T}$ is a 2,3-subgroup of (s) and $\mathfrak{I}$ contains a conjugate of $\mathfrak{B}$, then $\mathfrak{I}$ is contained in conjugate of $\mathfrak{R}$.

Proof. We assume without loss of generality that $\mathfrak{I}$ is a maximal 
2, 3-subgroup of $\mathfrak{B S}$, and that $\mathfrak{B} \subseteq \mathfrak{I}$. Since $\mathfrak{B}$ centralizes $\boldsymbol{O}_{2}(\mathfrak{T})$, it follows that $\boldsymbol{O}_{2}(\mathfrak{T})=1$, and so $\boldsymbol{O}_{3}(\mathfrak{I}) \neq 1$. Let $\mathfrak{I}_{3}$ be a $S_{3}$-subgroup of $\mathfrak{T}$. By maximality of $\mathfrak{T}, \mathfrak{I}_{3}$ is a $S_{3}$-subgroup of $N\left(O_{3}(\mathfrak{T})\right)$. We assume without loss of generality that $\mathfrak{I}_{3} \subseteq \mathfrak{P}$. This implies that $\mathfrak{B} \subseteq Z\left(O_{3}(\mathfrak{T})\right)$.

If $\mathfrak{I}$ contains a conjugate of $\mathfrak{D}$, we are done by Lemma 8.7. We therefore suppose that for each $G$ in $\left(\mathbb{B}, \mathfrak{D}^{a} \nsubseteq \mathfrak{T}\right.$.

Suppose $1 \leqq i \leqq 4$, and $\mathfrak{D}_{i} \cap \boldsymbol{O}_{3}(\mathfrak{I})=\mathfrak{D}_{i} \cap \mathfrak{I}_{3}$. We conclude that $\mathfrak{D}_{i} \subseteq \boldsymbol{O}_{3}(\mathfrak{T})$. Since $\mathfrak{D} \nsubseteq \mathfrak{T}_{3}$, we may choose $i$ with $1 \leqq i \leqq 4$ such that $\mathfrak{D}_{i} \cap \boldsymbol{O}_{3}(\mathfrak{I}) \subset \mathfrak{D}_{i} \cap \mathfrak{I}_{3}$. Set $\mathfrak{F}=\mathfrak{D}_{i} \cap \boldsymbol{O}_{3}(\mathfrak{T}), \mathfrak{F}^{*}=\mathfrak{D}_{i} \cap \mathfrak{I}_{3}$. The index $i$ is fixed in the following discussion. We note that $\mathfrak{F}$ and $\mathfrak{F}^{*}$ are normal elementary subgroups of $\mathfrak{I}_{3}$.

Let $\mathfrak{Z}$ be a $\mathfrak{F}^{*}$-invariant subgroup of $\boldsymbol{Q}_{3}^{1}(\mathfrak{T})$ minimal subject to $\left[\mathfrak{F}^{*}, \mathfrak{Q}\right] \neq 1$. Thus, $\mathfrak{F}^{*}$ acts irreducibly on the Frattini quotient group

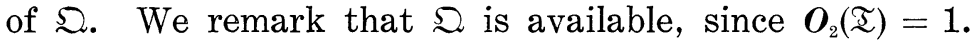

Let $\mathfrak{B}_{0}=\Omega_{1}\left(\boldsymbol{Z}\left(\boldsymbol{O}_{3}(\mathfrak{I})\right)\right)$, so that $\mathfrak{B} \subseteq \mathfrak{V}_{0}$.

Choose $Q \in \mathfrak{Q}-\mathfrak{Z}^{\prime}$. We will show that $\mathfrak{B}^{2} \cap \boldsymbol{C}\left(\mathfrak{D}_{i}\right)=1$. Suppose false, and that $B$ in $\mathfrak{B}^{*}$ satisfies $B^{Q} \in C\left(\mathfrak{D}_{i}\right)$. Hence, for $D$ in $\mathfrak{F}^{*}\left(\subseteq \mathfrak{D}_{i}\right)$, we have $B^{Q D}=B^{Q}$, or $B^{Q D Q^{-1}}=B$. Hence, $Q D Q^{-1} D^{-1}$ centralizes $B$ for each $D$ in $\mathfrak{F}^{*}$. This implies that $\Omega$ centralizes $B$. Apply Lemma 8.7 to $C(B)$ and conclude that if $\mathfrak{Q}=\mathfrak{\Omega}^{*} / \boldsymbol{O}_{3}(\mathfrak{I})$, then $\left[\mathfrak{\Omega}^{*}, \mathfrak{F}^{*}\right]$ is a

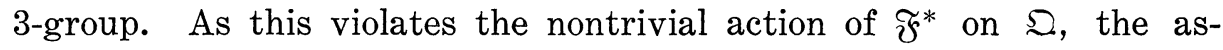
sertion follows.

Since $\mathfrak{B}^{Q} \subseteq O_{3}(\mathfrak{I}) \subseteq \mathfrak{I}_{3} \subseteq \mathfrak{P}$, we have $\mathfrak{B}^{Q} \subseteq N\left(\mathfrak{D}_{i}\right)$. Since $\mathfrak{D}_{i}$ is $3-$ reducible in $N\left(\mathfrak{D}_{i}\right)$, it follows that $\mathfrak{B}^{Q}$ is faithfully represented on $\mathbb{R}=$ $O_{3},\left(N\left(\mathfrak{D}_{i}\right) / C\left(\mathfrak{D}_{i}\right)\right)$. On the other hand, if $B \in \mathfrak{B}^{\#}$, then $\left[C(B)^{Q}, \mathfrak{B}^{Q}, \mathfrak{B}^{Q}\right]=1$. This implies that $\mathfrak{B}^{Q}$ centralizes every $2^{\prime}$-subgroup of $\mathbb{Z}$ which $\mathfrak{B}^{Q}$ normalizes. Thus, there is a 2-subgroup $\mathfrak{I}_{0}$ of $N\left(\mathfrak{D}_{i}\right)$ such that $\boldsymbol{A}_{N\left(\mathfrak{D}_{i}\right)}\left(\mathfrak{I}_{0}\right)$ contains a subgroup of type $(3,3)$. This violates Lemma 8.8 by $D_{2,3}$ in $N\left(D_{i}\right)$. The proof is complete.

LemMA 8.10. If $\sqrt{5}$ is any subgroup of (5) of type $(3,3)$, then (5) centralizes every abelian subgroup in $И(\mathfrak{S} ; 2)$.

Proof. Suppose $\mathfrak{Q}$ is a four-group in $И(\mathfrak{S} ; 2)$ with $[\mathfrak{Q}, \mathfrak{S}] \neq 1$. Let $\mathfrak{C}_{0}=C_{\mathfrak{G}}(\mathfrak{Q})$. Let $\mathfrak{I}$ be a $S_{2,3}$-subgroup of $C\left(\mathfrak{S}_{0}\right)$ which contains $\mathfrak{S} \mathfrak{D}$. By Lemma 8.9, $\mathfrak{T}^{G} \subseteq \mathfrak{N}$ for some $G$ in (3). Lemma 8.8 (iv) is violated.

Lemma 8.11. Hypothesis 7.2 is satisfied with $p=2$. Furthermore, $\mathfrak{M}$ has the following properties:

(i) $S_{3}$-subgroups of $\mathfrak{M}$ are noncyclic.

(ii) $\mathfrak{M}$ is a 2, 3-group.

(iii) $\mathfrak{M}$ contains no elementary subgroup of order 27.

(iv) $m\left(\mathfrak{M}_{0}\right) \leqq 2$ for every 3-subgroup $\mathfrak{M}_{0}$ of $\mathfrak{M}$. 
Proof. Let $\mathfrak{T}$ be a 2, 3-subgroup of $(\$)$ which contains elements of $\mathscr{T}(2)$ and $\mathscr{T}(3)$; $\mathfrak{I}$ is available by hypothesis (vii) of Theorem 8.1. We assume without loss of generality that $\mathfrak{T}$ is a maximal 2,3-subgroup of (3). By Lemma 8.8 (i), $\mathfrak{I}$ is contained in no conjugate of $\mathfrak{R}$. By Lemma 8.9, $\mathfrak{I}$ contains no conjugate of $\mathfrak{B}$. This fact together with maximality of $\mathfrak{I}$ implies that $\boldsymbol{O}_{3}(\mathfrak{I})=1$.

Let $\left(\mathfrak{S}\right.$ be a subgroup of $\mathfrak{I}$ of type $(3,3)$ and let $\mathfrak{I}_{3}$ be a $S_{3}$-subgroup of $\mathfrak{I}$ containing $\mathfrak{C}$. By Lemma 8.10, $\mathfrak{C}$ centralizes $\boldsymbol{Z}\left(\boldsymbol{O}_{2}(\mathfrak{I})\right)$. Hence, $\Omega_{1}\left(\mathfrak{I}_{3}\right)$ centralizes $\boldsymbol{Z}\left(\boldsymbol{O}_{2}(\mathfrak{I})\right)$. By Lemma 8.9, each $S_{2,3}$-subgroup of $N\left(\Omega_{1}\left(\mathfrak{I}_{3}\right)\right)$ is contained in a conjugate of $\mathfrak{R}$. Hence, $\mathfrak{I}_{2}$ centralizes $\boldsymbol{Z}\left(\boldsymbol{O}_{2}(\mathfrak{I})\right)$ by Lemma 8.8 (iv). By hypothesis (iv) of Theorem 8.1, $\mathfrak{I} \cdot \boldsymbol{C}\left(\boldsymbol{Z}\left(\boldsymbol{O}_{2}(\mathfrak{T})\right)\right.$ is solvable, so by maximality of $\mathfrak{I}$, we conclude that $\mathfrak{T}$ is a $S_{2,3}$-subgroup of $\mathfrak{I} \boldsymbol{C}\left(\boldsymbol{Z}\left(\boldsymbol{O}_{2}(\mathfrak{I})\right)\right)$. Hence, we can choose a $S_{2}$-subgroup $\mathfrak{P}_{2}$ of $\mathfrak{S}_{3}$ such that $\mathfrak{P}_{2} \cap \mathfrak{I}=\mathfrak{T}_{2}$ is a $S_{2}$-subgroup of $\mathfrak{I}$, and be guaranteed that $\boldsymbol{Z}\left(\mathfrak{P}_{2}\right) \subseteq \boldsymbol{Z}\left(\boldsymbol{O}_{2}(\mathfrak{I})\right)$. Hence, $\mathfrak{I} \subseteq \boldsymbol{C}\left(\boldsymbol{Z}\left(\mathfrak{P}_{2}\right)\right)$, so by maximality of $\mathfrak{I}$, we have $\mathfrak{I}_{2}=\mathfrak{P}_{2}$.

By Lemma 7.4, $\Omega_{1}\left(\boldsymbol{Z}\left(\boldsymbol{O}_{2}(\mathfrak{T})\right)\right)=\Omega_{1}\left(\boldsymbol{Z}\left(\mathfrak{I}_{2}\right)\right)$ is of order 2 and

$$
N\left(\Omega_{1}\left(Z\left(\mathfrak{I}_{2}\right)\right)\right)=\mathfrak{M} \in \mathscr{M C S}(G) .
$$

By construction, $\mathfrak{I}_{3} \subseteq \mathfrak{M}$, so (i) is satisfied. By Lemma 7.5, $\boldsymbol{O}_{2}(\mathfrak{M})=\mathfrak{F}$ is of symplectic type with $w \leqq 4$. (ii) is an easy consequence of this fact together with (i).

Suppose $\mathfrak{F}$ is an elementary subgroup of $\mathfrak{M}$ of order 27. Clearly, the width of $\mathfrak{S}$ is at least 3 . By Lemma 7.5, no element of $\mathfrak{F}^{\sharp}$ centralizes any four-subgroup of $\mathfrak{S}$. This is obviously impossible.

It remains to prove (iv). By Lemma 7.5 (c), $\mathfrak{M}_{0}$ is isomorphic to a subgroup $\mathfrak{M}_{1}$ of $\left(Z_{3} \int Z_{3}\right) \times Z_{3}$. By Lemma 8.11 (iii), the intersection of $\mathfrak{M}_{1}$ with the normal abelian subgroup $\mathfrak{U}$ such that $m(\mathfrak{U})=4$ in $\left(Z_{3} \sim Z_{3}\right) \times Z_{3}$, is of order at most $3^{2}$. It follows that $\mathfrak{M}_{0}$ is either trivial, abelian of type $(3),(3,3)$ or $\left(3^{2}, 3\right)$, or non abelian of order $3^{3}$. In all cases, $m\left(\mathfrak{M}_{0}\right) \leqq 2$. The proof is complete.

Let $\mathfrak{C}$ be a subgroup of $\mathfrak{M}$ of type $(3,3)$, let $\mathfrak{I}_{3}$ be a $S_{3}$-subgroup of $\mathfrak{M}$ containing $\mathfrak{E}$, and let $\mathfrak{S}_{1}=\left[\mathfrak{F}_{\mathfrak{E}}, \mathfrak{E}\right]$, where $\mathfrak{S}=\boldsymbol{O}_{2}(\mathfrak{M})$. Let $I$ be the involution of $\mathfrak{S g}^{\prime}$. Choose $C$ in $\mathfrak{C}^{\#}$ so that $C_{\mathfrak{S}_{1}}(C)=\mathfrak{Q}$ is not centralized by $\mathfrak{C}^{5}$. We may assume that $C_{\mathfrak{M}}(C) \subseteq \mathfrak{N}$, since replacing $\mathfrak{M}$ by a suitable conjugate guarantees this. Let $\mathbb{Q}$ be a $S_{2,3}$-subgroup of $\mathfrak{R}$ containing $\mathfrak{S} \mathfrak{\Omega}$. This notation is fixed throughout the concluding argument.

LEMMA 8.12. (i) $\mathfrak{D}$ is a quaternion group.

(ii) $\mathfrak{R}$ is a 2,3-group.

(iii) $\mathfrak{R} \in \mathscr{L C S}$ (S) and $\mathfrak{R}$ is the only element of $\mathscr{L} S(\mathbb{S}$ (S) which contains $\mathfrak{R}$.

(iv) $\mathfrak{N}$ is the only element of $\mathscr{L S}(\mathbb{S})$ which contains $\mathfrak{E}$. 
Proof. Since $\mathfrak{K}_{1}$ is extra special, so is $\mathfrak{l}$. Since $\mathfrak{Q} \subseteq O^{3^{\prime}}(\mathfrak{R})$, Lemma 8.8 and Lemma 5.27 imply (i)

By (i) and Lemma 8.8, $\mathfrak{Z}$ is a $S_{2}$-subgroup of $\boldsymbol{O}^{3^{\prime}}(\mathfrak{R})$. Clearly, since $\mathfrak{M}$ is a 2, 3-group, $N_{\mathfrak{R}}(\mathfrak{Q})$ is a 2,3-group. Since $\mathfrak{C} \cong \subseteq O^{3^{\prime}}(\mathfrak{R})$, it follows that $\mathfrak{\subseteq} \boldsymbol{O}^{3^{\prime}}(\Re)^{\prime}$. Hence, $\mathfrak{Q}$ has a normal complement $\Re$ in $\boldsymbol{O}^{3^{\prime}}(\mathfrak{R})^{\prime}$. To prove (ii), it suffices to show that $\Re$ is a 3-group. Let $\Re_{0}$ be a $S_{3^{\prime}}$-subgroup of $\Re$ normalized by $\Omega$. Then $I$ inverts $\Re_{0}$ since $\mathfrak{M}$ is a 2, 3-group. Choose $\mathfrak{F} \operatorname{char} \boldsymbol{O}_{3}(\Re)$ with $\operatorname{ker}(\Re \rightarrow$ Aut $(\mathfrak{F}))$ a 3group, and with $\mathfrak{F}$ of exponent 3 . Such an $\mathfrak{F}$ is available by Lemma 5.18 and 0.3.6. As $\cong$ is nonabelian, $\Re_{0}$ is noncyclic. It follows readily that $I$ centralizes a subgroup of $\mathfrak{F} / \boldsymbol{D}(\mathfrak{F})$ of order 27. This implies that $\boldsymbol{C}_{\widetilde{F}}(I)$ contains an elementary subgroup of order 27 , in violation of Lemma 8.11. (ii) is proved.

Let $\mathfrak{P} \cong \mathfrak{R}_{1} \in \mathscr{L C S}(\mathbb{S}) . \quad$ By Hypothesis 8.2, it suffices to show that $C_{\mathfrak{\Re}_{1}}(\widetilde{\mathfrak{B}}) \leqq \Re$, where $\widetilde{\mathfrak{B}}=\Omega_{1}(\boldsymbol{Z}(\mathfrak{P}))^{\mathfrak{R}_{1}}$. Since $\boldsymbol{C}_{\mathfrak{R}_{1}}(\widetilde{\mathfrak{B}}) \subseteq N\left(\Omega_{1}(\boldsymbol{Z}(\mathfrak{P}))\right)$, and since $I \in N(\mathfrak{P}) \subseteq N\left(\Omega_{1}(Z(\mathfrak{P}))\right)$, we may replace $\mathfrak{N}_{1}$ by an element of ((S) which contains $N\left(\Omega_{1}(Z(\mathfrak{P}))\right)$ and so assume that $I \in \Re_{1}$.

Let $\&$ be a $S_{3^{\prime}}$-subgroup of $\mathfrak{R}_{1}$ which contains $I$. By Lemma 8.8 (i) and Lemma 8.9, it follows that $\&$ has a normal 2-complement $\Re$. Since $\mathfrak{M}$ is a 2,3-group, $I$ inverts $\Re$. Suppose by way of contradiction that $\Omega \neq 1$. Since $O_{3^{\prime}}\left(\mathfrak{N}_{1}\right)=1, \Omega\langle I\rangle$ is faithfully represented as automorphisms of $O_{3}\left(\Re_{1}\right)$. By Lemma 8.11 (iv), the only possibility is that $|\Re|=5$, that $\boldsymbol{C}(\Re) \cap \boldsymbol{O}_{3}\left(\mathfrak{N}_{1}\right) \supseteqq D\left(\boldsymbol{O}_{2}\left(\mathfrak{N}_{1}\right)\right)$ and that $\boldsymbol{O}_{3}\left(\mathfrak{N}_{1}\right) / \boldsymbol{C}(\Re) \cap \boldsymbol{O}_{3}\left(\mathfrak{N}_{1}\right)$ is elementary of order $3^{4}$. Since $\Re$ is an $S$-subgroup of $\Re_{1}$, it follows that $\Re_{5^{\prime}}\left(\Re_{1}\right)$ / $\boldsymbol{O}_{5^{\prime}}\left(\mathfrak{R}_{1}\right)$ is a chief factor of $\mathfrak{R}_{1}$. Hence, $I \notin \mathfrak{R}_{1}^{\prime}$. This implies that $O_{3}\left(\mathfrak{N}_{1}\right)=\mathfrak{P}$, so that $\mathfrak{N}_{1} \subseteq N(\mathfrak{B})=\mathfrak{R}$. Hence, $\mathfrak{N}_{1}=\mathfrak{R}$. This is absurd since $I \in \mathfrak{N}^{\prime}$. This contradiction forces $\Re=1$, that is, $\mathfrak{R}_{1}$ is a 2, 3-group.

Since $\left|\boldsymbol{C}\left(\Omega_{1}(\boldsymbol{Z}(\mathfrak{P}))\right)\right|$ is odd, it follows that $\boldsymbol{C}_{\mathfrak{R}_{1}}(\widetilde{\mathfrak{B}})=\boldsymbol{C}_{\mathfrak{B}}(\widetilde{\mathfrak{B}}) \subseteq \mathfrak{P} \leqq \mathfrak{N}$. Thus, (iii) holds.

We turn to (iv). Let $\mathscr{P}=\left\{\mathfrak{P}_{0} \mid\right.$ (i) $\mathfrak{P}_{0}$ is a 3-subgroup of $N$, (ii) $\mathfrak{P}_{0} \supseteqq$ $\mathfrak{B}^{N}$ for some $N$ in $\mathfrak{R}$, (iii) $\mathfrak{P}_{0}$ is contained in a solvable subgroup of (S) which is not contained in $\mathfrak{R}$.\}. Suppose by way of contradiction that $\mathscr{P} \neq \varnothing$. Choose $\mathfrak{P}_{0}$ in $\mathscr{P}$ with $\left|\mathfrak{P}_{0}\right|$ maximal. We assume without loss of generality that $\mathfrak{P}_{0} \subseteq \mathfrak{P}$. Let $\Re$ be a solvable subgroup of $(B)$ which contains $\mathfrak{P}_{0}$ and is minimal subject to $\Re \nsubseteq \Re$. Since $\mathfrak{P} \notin \mathscr{P}$, it follows that $\mathfrak{P}_{0} \subset \mathfrak{P}$, so maximality of $\left|\mathfrak{P}_{0}\right|$ forces $\mathbb{N}_{\mathfrak{s}}\left(\mathfrak{B}_{0}\right) \notin \mathscr{P}$. In particular, $N\left(\mathfrak{B}_{0}\right) \subseteq \Re$. This implies that $\mathfrak{P}_{0}$ is a $S_{p}$-subgroup of $\Re$. Minimality of $\Omega$ yields that $\Re=\Re_{0} \Re_{1}$, where $\Re_{1}$ is a $q$-group for some prime $q \neq 3$.

Since $\mathfrak{B}^{N} \subseteq \mathfrak{B}_{0}$ for some $N$ in $\mathfrak{R}$, it follows that $\boldsymbol{O}_{3^{\prime}}(\Re) \subseteq \mathfrak{R}$, as $\boldsymbol{O}_{3^{\prime}}(\Re)$ is generated by its subgroups $\boldsymbol{O}_{3^{\prime}}(\Re) \cap C(B), B \in\left(\mathfrak{B}^{N}\right)^{*}$.

Suppose $q=2$. Then by Lemma 8.9, $\Re \subseteq \mathfrak{R}^{G}$ for some $G$ in (S). Hence $\mathfrak{P}_{0} \subseteq \mathfrak{R}^{\alpha}$. Let $\mathfrak{P}^{*}$ be a $S_{3}$-subgroup of $\mathfrak{R} \cap \mathfrak{R}^{G}$ which contains $\mathfrak{P}_{0}$. 
Maximality of $\left|\mathfrak{P}_{0}\right|$ forces $\mathfrak{P}_{0}=\mathfrak{P}^{*}$. But then since $N\left(\mathfrak{B}_{0}\right) \subseteq \mathfrak{N}$, we get that $\mathfrak{B}_{0}$ is a $S_{3}$-subgroup of $\mathfrak{N}^{G}$. This is absurd. Hence, $q \neq 2$.

It is a consequence of [43] that $\Re=\boldsymbol{O}_{3^{\prime}}(\Re) \mathfrak{U}_{1} \mathfrak{A}_{2}$, where

$$
\mathfrak{A}_{1}=C_{\Re}\left(Z\left(\mathfrak{P}_{0}\right)\right), \mathfrak{A}_{2}=N_{\Re}\left(J\left(\mathfrak{P}_{0}\right)\right) \text {. }
$$

Maximality of $\left|\mathfrak{P}_{0}\right|$ forces $N\left(Z\left(\mathfrak{P}_{0}\right)\right) \subseteq \mathfrak{R}, N\left(J\left(\mathfrak{P}_{0}\right)\right) \subseteq \mathfrak{N}$, so $\mathfrak{\Re} \cong \mathfrak{R}$. This establishes (iv).

We may now complete the proof of Theorem 8.1. Choose $C_{1}$ in ${ }^{\sharp}$. Then $\boldsymbol{C}\left(C_{1}\right) \supseteqq \mathfrak{B}$, so that $\boldsymbol{C}\left(C_{1}\right) \subseteq \mathfrak{R}$. Hence, $\mathfrak{S} \subseteq \mathfrak{N}$, in violation of Lemma 8.8 (ii).

\section{A characterization of $S_{4}(3)$.}

THEOREM 9.1. $\quad S_{4}(3)$ is the only simple group \& with the following properties:

( i ) (S) contains an elementary subgroup of order 27.

( ii ) If $\mathfrak{P}$ is a $S_{3}$-subgroup of $\mathbb{S}$ and $\mathfrak{2} \in \mathscr{S}_{\text {cre }}(\mathfrak{\$})$, then $\mathrm{U}(\mathfrak{Q})$ is trivial.

(iii) The center of a $S_{3}$-subgroup of \&s is cyclic.

(iv) The normalizer of every nonidentity 3-subgroup of (S) is solvable.

( v ) $S_{2}$-subgroups of $\$$ s contain normal elementary subgroups of order 8.

( vi ) If $\mathfrak{I}$ is a $S_{2}$-subgroup of $\mathbb{( S )}$ and $\mathfrak{B} \in \mathscr{S}_{\text {cne } 3}(\mathfrak{T})$, then $\mathrm{U}(\mathfrak{B})$ is trivial.

(vii) The centralizer of every involution of (B) is solvable.

(viii) $2 \sim 3 . \quad$ (See Definition 2.9.).

After careful translation, it can be shown that Dickson [12] lists several properties of $S_{4}(3)$. Namely, $A(4,3)$ is Dickson's notation for $S_{4}(3)$ (pp. 89-100). Now in $\S 194$ (pp. 109-191), Dickson sets $F O\left(m, p^{n}\right)=$ $\boldsymbol{O}_{1}^{\prime}\left(m, p^{n}\right)$ (for $m$ odd), so by $\S 189$ (pp. 179-183), $A(4,3) \cong F O(5,3) \cong$ $S_{4}(3)$. Thus, by $\S 270$ (pp. 292-293), $S_{4}(3)$ has a subgroup of index 27 which is a split extension of an elementary group of order 16 by $A_{5}$. So $S_{4}(3)$ is not an $N$-group. That $S_{4}(3)$ satisfies the hypothesis of Theorem 9.1 is left as an exercise. We remark that (viii) holds for $S_{4}(3)$, the centralizers of suitable involutions exhibiting $2 \sim 3$.

Throughout most of this section the following notation is used: $\mathfrak{P}$ is a $S_{3}$-subgroup of $\mathbb{B}$,

$$
3=\Omega_{1}(Z(\mathfrak{P})), \mathfrak{R}=N(3), \mathfrak{K}=\boldsymbol{O}_{3}(\mathfrak{R}) .
$$

By hypothesis (iii), $|3|=3$, and by hypothesis (ii), $\boldsymbol{O}_{3^{\prime}}(\mathfrak{N})=1$. By hypothesis (iv), $\mathfrak{R}$ is solvable, so by Lemma 1.2 .3 of [26], $\boldsymbol{C}_{\mathfrak{R}}(\mathfrak{S})=\boldsymbol{Z}(\mathfrak{S})$. 
Clearly, $\boldsymbol{C}_{\mathfrak{R}}(\mathfrak{K})=\boldsymbol{C}(\mathfrak{K})$.

We remark that (S) satisfies Hypothesis 7.4 and also satisfies Hypothesis 7.1 for $p=2$ and for $p=3$.

Hypothesis 9.1. $\quad \mathfrak{S}$ is the central product of a cyclic group and a nonabelian group of order 27 and exponent 3.

Lemma 9.1. Assume that Hypothesis 9.1 is satisfied. Then

(i ) $|\mathfrak{B}: \mathfrak{S} \mathfrak{E}|=3$.

(ii) $|\mathfrak{S}|=27$.

(iii) $\quad O^{3^{\prime}}(\Re) / \mathfrak{S} \cong S L(2,3)$.

Proof. We remark that $G L(2,3)$ contains no noncyclic abelian subgroup of order 8 .

As $\mathfrak{R} / \mathfrak{S}_{\mathfrak{C}}$ is faithfully represented on $\Omega_{1}(\mathfrak{S}) / \boldsymbol{D}\left(\Omega_{1}\left(\mathfrak{S}_{\mathfrak{C}}\right)\right)$, it follows that $\mathfrak{N}$ is a 2,3-group, and $\mathfrak{R} / \mathfrak{F}$ is isomorphic to a subgroup of $G L(2,3)$. As $\mathfrak{S}$ contains no elementary subgroup of order 27 and $\mathfrak{P}$ does, (i) holds.

Let $\mathfrak{A}$ be a normal elementary subgroup of $\mathfrak{P}$ of order 27 . Since $|\mathfrak{P}: \mathfrak{S}|=3$, it follows that $\boldsymbol{O}^{3^{\prime}}(\mathfrak{N}) / \mathfrak{S} \cong S L(2,3)$, yielding (iii). Let $\mathfrak{Q}$ be a $S_{2}$-subgroup of $\boldsymbol{O}^{3^{\prime}}(\mathfrak{R})$ so that $\mathfrak{Q}$ is a quaternion group. Let $\mathfrak{A}_{0}=$ $\mathfrak{A} \cap \mathfrak{F}$. Let $I$ be the involution of $\Omega$. As $I$ inverts every element of $\Omega_{1}(\mathfrak{E}) / 3$, it folllows that $I$ normalizes $\mathfrak{A}_{0}$. Since $I$ also normalizes $\mathfrak{P}$, it follows that $I$ normalizes $\boldsymbol{C}_{\mathfrak{B}}\left(\mathfrak{H}_{0}\right)=\langle\mathfrak{A}, \boldsymbol{Z}(\mathfrak{F})\rangle$. Hence, $I$ normalizes $\Omega_{1}\left(\boldsymbol{C}_{\mathfrak{B}}\left(\mathfrak{H}_{0}\right)\right)=\mathfrak{A}$. Since $I$ centralizes the factor $\boldsymbol{O}^{3^{\prime}}(\mathfrak{R}) / \boldsymbol{O}^{3^{\prime}}(\mathfrak{N})^{\prime} \mathfrak{S} \cong \mathfrak{F} / \mathfrak{F}$, it follows that $\mathfrak{A}-\mathfrak{U}_{0}$ contains an element $A_{1}$ such that $A_{1}^{I}=A_{1}$. Since $I$ also centralizes 3 , it follows that $C_{\mathfrak{P}}(I)=\left\langle A_{1}\right\rangle \times 3=\mathfrak{A}_{1}$. Also, $\boldsymbol{C}_{\mathfrak{\beta}}(I)=\mathfrak{U}_{1} \mathcal{Z}_{1}$, where $\mathfrak{Z}_{1}=\boldsymbol{Z}(\mathfrak{K})$, and it is clear that $\boldsymbol{C}_{\mathfrak{P}}(I)$ is a $S_{3^{-}}$ subgroup of $C_{\mathfrak{R}}(I)$.

Suppose $\left|Z_{1}\right|>3$. Thus, $|\mathfrak{P}|>3^{4}$, so Lemma 7.6 is at our disposal.

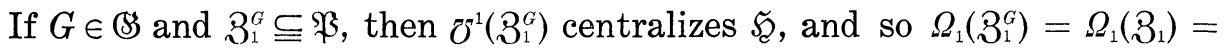
3 , so that $G \in \mathfrak{N}, 3_{1}^{G}=Z_{1}$. We may therefore apply Theorem 14.4.2 of [21] and conclude that $\mathfrak{B} \subseteq \mathfrak{R}^{\prime}$. Since Aut $\left(B_{1}\right)$ is abelian, this implies that $Z_{1}=\boldsymbol{Z}(\Re)$. We may therefore appeal to Lemma $7.6(\mathrm{~d})$ and conclude that if $\mathfrak{U}^{*}$ is any subgroup of $\mathfrak{A}$ of type $(3,3)$, then $\mathfrak{U}^{*}$ centralizes every element of $\boldsymbol{U}\left(\mathfrak{U}^{*} ; 2\right)$. Taking $\mathfrak{A}^{*}=\mathfrak{A}_{1}$, Lemma 7.4 is violated. This completes the proof of (ii).

Lemma 9.2. Assume that Hypothesis 9.1 is satisfied. Let $\mathfrak{U} \in \mathscr{S}_{\text {cnz }_{3}}(\mathfrak{P})$ and let $I$ be an involution of $\mathfrak{N}^{\prime}$. Then

(i) $S_{2}$-subgroups of $\mathfrak{N}$ are quaternion.

(ii) If $\mathfrak{A}_{0}=C_{\mathfrak{Q}}(I)$, then

(a) $\left|\mathfrak{A}_{0}\right|=9$.

(b) $\mathfrak{U}_{0}$ contains a subgroup $\mathfrak{X}_{1}$ of order 3 such that $\boldsymbol{C}\left(\mathfrak{U}_{1}\right) \nsubseteq \mathfrak{N}$. 
(iii) If $\mathfrak{M}=C(I)$, then $\boldsymbol{O}_{2}(\mathfrak{M})$ is extra special of width $2, \boldsymbol{O}_{2^{\prime}}(\mathfrak{M})=$ 1, and $\left|\mathfrak{M}: \mathrm{O}_{2}(\mathfrak{M})\right|_{2}=2$.

(iv) $\boldsymbol{A}_{\mathscr{S}}(\mathfrak{U}) \cong \Sigma_{4}$.

Proof. Let $\cong$ be a $S_{2}$-subgroup of $O^{3^{\prime}}(\mathfrak{R})$. By Lemma $9.1(\mathrm{i})$, $\mathfrak{}$ is a quaternion group. It clearly suffices to prove the lemma on the assumption that $I$ is the involution of $\cong$.

By Lemma 9.1 and hypothesis (i) of Theorem 9.1, the group $\mathfrak{P}$ is $Z_{3} \int Z_{3}$. Hence, $\mathfrak{A}$ char $\mathfrak{P}$. Since $I$ normalizes $\mathfrak{P}$, it therefore normalizes $\mathfrak{A}$. This implies (ii)(a), since $I$ centralizes $\boldsymbol{Z}(\mathfrak{C})$ and $\boldsymbol{O}^{3^{\prime}}(\mathfrak{R}) / \boldsymbol{O}^{3^{\prime}}(\mathfrak{R})^{\prime}$.

Clearly, $\mathfrak{M}$ contains an element of $\mathscr{\mathscr { C }}(2)$. It is equally clear from $(B)$ and Lemma 9.1 that

$(*)$

if $\mathfrak{X}$ is any noncyclic subgroup of $\mathfrak{A}$, then $\mathfrak{X}$ centralizes every abelian subgroup of $h(\mathfrak{X} ; 2)$.

Let $\mathfrak{I}$ be a $S_{2,3}$-subgroup of $\mathfrak{M}$ which contains $\left\langle\mathfrak{U}_{0}, \mathfrak{Q}\right\rangle$. Let $\mathfrak{I}_{2}$ be a $S_{2}$-subgroup of $\mathfrak{I}$ which contains $\mathfrak{\Omega}$. We may apply Lemma 7.5 with $\mathfrak{A}_{0}$ in the role of $\mathfrak{B}$. Thus, there is an element $\widetilde{\mathfrak{M}}$ of $\mathscr{A S}(\mathbb{S})$ satisfying the conclusions of Lemma 7.5. By Lemma 7.5 (e), we get $\mathfrak{Q} \leqq \boldsymbol{O}_{2}(\widetilde{\mathfrak{M}})$. Hence, $\langle I\rangle \triangleleft \widetilde{\mathfrak{M}}$, so $\widetilde{\mathfrak{M}}=C(I)=\mathfrak{M}$. Since $\langle I\rangle$ is a $S_{2}$ subgroup of $\boldsymbol{C}\left(\mathfrak{A}_{0}\right)$, it follows that

$$
\boldsymbol{O}_{2}(\mathfrak{M}) \text { is extra special of width } w=2,3 \text {, or } 4 \text {. }
$$

Thus, (ii)(b) holds.

Again, let $\mathfrak{X}$ be a noncyclic subgroup of $\mathfrak{A}$. Suppose that $|\boldsymbol{C}(\mathfrak{X}) \cap N(\mathfrak{U})|$ is even. Then of course $|\mathfrak{X}|=9$, as $\mathfrak{A}$ is a self-centralizing subgroup of $\mathbb{S}$. Let $J$ be an involution of $C(\mathfrak{X}) \cap N(\mathfrak{U})$. Then (*) and Lemma 7.5 yield that $J$ and $I$ are conjugate in $\mathbb{S}$. Since $\mathfrak{X}$ is faithfully represented on $\boldsymbol{O}_{2}(\boldsymbol{C}(J))$, we can choose a subgroup $\mathfrak{Y}$ of $\mathfrak{X}$ of order 3 such that

$$
\mathfrak{X} \text { does not centralize } \boldsymbol{C}(\mathfrak{Y}) \cap \boldsymbol{O}_{2}(\boldsymbol{C}(J)) \text {. }
$$

Thus, $\boldsymbol{C}(\mathfrak{Y})$ is not 3-closed. Thus, $\mathfrak{A}$ is not a $S_{3}$-subgroup of $\boldsymbol{C}(\mathfrak{Y})$ ). This implies that

$$
C\left(\mathfrak{Y )} \text { contains a } S_{3} \text {-subgroup of }\right. \text { (S) . }
$$

Let $\tilde{\mathfrak{P}}$ be a $S_{3}$-subgroup of $\boldsymbol{C}(\mathfrak{Y})$ which contains 2 . Thus $\langle\tilde{\mathfrak{P}}, J\rangle \subseteq$ $\boldsymbol{C}(\mathfrak{Y})$. Thus, $J$ normalizes both $\mathfrak{U}$ and $\boldsymbol{O}_{3}(\boldsymbol{C}(\mathfrak{Y}))$, so $J$ normalizes $\left\langle\mathfrak{A}, \boldsymbol{O}_{3}(\boldsymbol{C}(\mathfrak{Y}))\right\rangle$. Thus, Lemma 9.1 yields that

if $\mathfrak{X}$ is any noncyclic subgroup of $\mathfrak{A}$, then each involution of $C(\mathfrak{X}) \cap N(\mathfrak{U})$ normalizes some $S_{3}$-subgroup of $N(\mathfrak{U})$. 
By (9.3) with the pair $\left(\mathfrak{A}_{1}, \mathfrak{A}_{0}\right)$ in the role of $(\mathfrak{Y}, \mathfrak{X})$, we conclude that $C\left(\mathfrak{U}_{1}\right)$ contains a $S_{3}$-subgroup $\mathfrak{P}^{*}$ of $\mathbb{S}$ with $\mathfrak{A} \subset \mathfrak{P}^{*}$. Hence, $N(\mathfrak{U})$ is not 3 -closed, since $\mathfrak{P}$ and $\mathfrak{P}^{*}$ are distinct $S_{3}$-subgroups of $N(\mathfrak{A})$.

Set $\tilde{\mathfrak{N}}=N(\mathfrak{U})$. Clearly,

$$
\mathfrak{A}=\boldsymbol{O}_{3}(\tilde{\mathfrak{N}})=C(\mathfrak{A}), 1=\boldsymbol{O}_{3^{\prime}}(\tilde{\mathfrak{N}}), I \in \tilde{\mathfrak{N}} .
$$

Suppose 13||$\tilde{\mathfrak{N}} \mid$. Since $I \in \tilde{\mathfrak{N}}$, it follows that $I$ centralizes a $S_{1^{-}}$ subgroup of $\tilde{\mathfrak{N}}$, since the nonidentity 13-elements of $G L(3,3)$ are nonreal. However, $13 \nmid|\mathfrak{M}|$, since $4 \geqq w$. Hence, $\tilde{\mathfrak{N}}$ is a 2,3 -group.

Let $\mathfrak{I}_{0}$ be a $S_{2}$-subgroup of $\boldsymbol{O}_{3,2}(\tilde{\mathfrak{R}})$, and let $\Omega=N_{\tilde{\mathfrak{N}}}\left(\mathfrak{T}_{0}\right)$. Thus, $\tilde{\mathfrak{R}}=\mathfrak{U} \Re, \mathfrak{A} \cap \mathfrak{R}=1$, so that $\mathfrak{R} \cong \boldsymbol{A}_{\mathfrak{S}}(\mathfrak{A})$. Suppose $J$ is an involution of $\mathfrak{I}_{0}$ and $\mathfrak{A} \cap C(J)=\mathfrak{X}$ is noncyclic. Thus, $|\mathfrak{X}|=9$. By (9.4), $J$ normalizes some $S_{3}$-subgroup $\tilde{\mathfrak{R}}$ of $\tilde{\mathfrak{R}}$. Since $\tilde{\mathfrak{R}} \supset \mathfrak{A}, \tilde{\mathfrak{R}} \cap \Re$ is of order 3 . Hence, $[\tilde{\mathfrak{R}} \cap \Omega, J] \subseteq \mathfrak{I}_{0} \cap \tilde{\mathfrak{P}}=1$, so that $\tilde{\mathfrak{P}} \cap \Re$ centralizes $J$. Hence, $\tilde{\mathfrak{P}} \cap \Re$ normalizes $\mathfrak{X}$, that is, $\mathfrak{X} \triangleleft \tilde{\mathfrak{P}}$. Hence, $\mathfrak{X} \in \mathscr{K}(3)$. Thus, $J$ centralizes elements of $\mathscr{C}(3)$ and $\mathscr{C}(2)$. This violates the solvability of $C(J)$. Hence,

no element of $\mathfrak{T}_{0}^{\sharp}$ centralizes a noncyclic subgroup of $\mathfrak{A}$.

Since $|\boldsymbol{Z}(\mathfrak{P})|=3, \mathfrak{R} \cap \Re$ is indecomposable on $\mathfrak{A}$. Hence, $\mathfrak{I}_{0}$ acts on $\mathfrak{U}$ as a multiple of the sum of the $\Re$-conjugates of a fixed $F_{3^{-}}$irreducible representation $\rho$. If $\mathfrak{I}_{0}$ is nonabelian, then 2 divides $\operatorname{deg} \rho$. Hence, 2 divides $3=m(\mathfrak{A})$, a contradiction. Hence, $\mathfrak{I}_{0}$ is abelian. If $\mathfrak{I}_{0}$ is not elementary, then $\operatorname{deg} \rho \neq 1$ or 3 . So $\operatorname{deg} \rho=2$, which again gives a contradiction. Hence, $\mathfrak{I}_{0}$ is elementary. Now (9.5) implies that $\left|\mathfrak{I}_{0}\right| \leqq 4$, so we must have equality, since $\mathfrak{I}_{0}=\boldsymbol{O}_{2}(\mathfrak{R})$ and $|\mathfrak{P} \cap \mathfrak{R}|=$ 3. Since $I \in \mathfrak{R} \cap N(\mathfrak{P})$, it follows that $\Re \cong \Sigma_{4}$, which establishes (iv) and also (i).

It remains to show that $w=2$ and that $\left|\mathfrak{M}: \boldsymbol{O}_{2}(\mathfrak{M})\right|_{2}=2$, since by (9.1) we know that as $\boldsymbol{O}_{2}(\mathfrak{M})$ is extra special.

Suppose $\mathfrak{A}$ is any subgroup of $\mathfrak{A}$ of order 3 which is conjugate

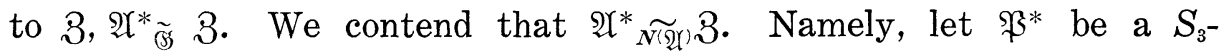
subgroup of $\boldsymbol{C}\left(\mathfrak{A}^{*}\right)$ which contains $\mathfrak{A}^{\text {. Then }} \mathfrak{P}$ and $\mathfrak{P}^{*}$ both normalize $\mathfrak{A}$, since $\left|\mathfrak{P}^{*}: \mathfrak{U}\right|=3$. We may thus choose $N$ in $N(\mathfrak{A})$ such that $\mathfrak{P}^{* N}=\mathfrak{P}$; since $3=\boldsymbol{Z}(\mathfrak{P})$, we necessarily have $\mathfrak{A}^{* N}=3$, as desired.

Since $\mathfrak{P}\langle I\rangle$ normalizes 3 , we obtain all $N(\mathfrak{U})$-conjugates of 3 by transformation with elements of $\mathfrak{I}_{0}$. We will show that 3 and $\mathfrak{I}_{1}$ are the only $N(\mathfrak{H})$-conjugates of 3 which are in $\mathfrak{U}_{0}$. If $K \in \mathfrak{I}_{0}^{\sharp}$ and $\mathfrak{Z}^{K} \subseteq \mathfrak{A}_{0}$, then since no element of $\mathfrak{I}_{0}^{\sharp}$ normalizes 3 , we conclude that $K$ normalizes $\mathfrak{A}_{0}$. It is clear that $\mathfrak{I}_{0}$ does not normalize $\mathfrak{A}_{0}$, so our assertion follows. It is an immediate consequence of the preceding paragraph that $w=2$. That is, only 3 and $\mathfrak{A}_{1}$ centralize elements of $\boldsymbol{O}_{2}(\mathfrak{M})-\langle I\rangle$. Since $N\left(\mathfrak{U}_{0}\right) \subseteq N(\mathfrak{U})$, we have $\left|\mathfrak{M}: O_{2}(\mathfrak{M})\right|_{2}=2$, and the proof is complete. 
We now change notation somewhat in order to conform with more standard notation. Let $\mathfrak{B}_{1}=N(\mathfrak{3}), \mathfrak{B}_{2}=N(\mathfrak{A})$, and let $\mathfrak{B}=\mathfrak{P}\langle I\rangle, \mathfrak{S}=$ $\langle I\rangle$. Let $\mathfrak{\Omega}_{1}$ be a $S_{2}$-subgroup of $\mathfrak{B}_{1}$ which contains $I$, and let $\mathfrak{Q}_{2}$ be a $S_{2}$-subgroup of $\mathfrak{B}_{2}$ which contains $I$. Thus, $\mathfrak{\Omega}_{1}$ is a quaternion group and $\mathfrak{\Omega}_{2}$ is a dihedral group of order 8 . Let $\mathfrak{I}_{2}=\mathfrak{\Omega}_{2} \cap \boldsymbol{O}^{3^{\prime}}\left(\mathfrak{B}_{2}\right)$, so that $\mathfrak{I}_{2}$ is a four-group.

Let $\mathfrak{F}_{2}=N_{\mathfrak{B}_{2}}\left(\mathfrak{I}_{2}\right)$. Thus, $\mathfrak{F}_{2}$ is a complement to $\mathfrak{A}$ in $\mathfrak{B}_{2}$ and $\mathfrak{F}_{2} \cong$ $\Sigma_{4}$. Let $\mathfrak{X}_{1}=\mathfrak{P} \cap \mathfrak{E}_{2}$, so that $\mathfrak{X}_{1}$ is of order 3 and is inverted by $I$. Since $\boldsymbol{O}_{3}\left(\mathfrak{B}_{1}\right)$ contains all elements of $\mathfrak{P}$ which are inverted by $I$, we have $\mathfrak{X}_{1} \subseteq O_{3}\left(\mathfrak{B}_{1}\right)$. Since $\mathfrak{Q}_{1}$ permutes transitively the subgroups of $\boldsymbol{O}_{3}\left(\mathfrak{B}_{1}\right) / 3$ of order 3 , we may choose $Q$ in $\mathfrak{\Omega}_{1}$ so that $\mathfrak{X}_{2}=\mathfrak{X}_{1}^{Q}$ lies in $\mathfrak{A}$. Thus, $I$ inverts $\mathfrak{X}_{2}$, since $Q$ centralizes $I$. Let $\langle J\rangle=\mathfrak{I}_{2} \cap C(I)$, that is, let $J$ be a generator for $\boldsymbol{Z}\left(\mathfrak{\Omega}_{2}\right)$. Since $\mathfrak{A} \cap \boldsymbol{C}(I)$ is of order $9, \mathfrak{X}_{2}$ is the only subgroup of $\mathfrak{A}$ of order 3 which is inverted by $I$, so that $\mathfrak{X}_{2}^{J}=\mathfrak{X}_{2}$. Let $\mathfrak{X}_{4}=3$ and set $\mathfrak{X}_{3}=\mathfrak{X}_{4}^{J}$. We may now draw up the following table:

\begin{tabular}{c|c|c} 
& $J$ & $Q$ \\
\hline $\mathfrak{X}_{1}$ & - & $\mathfrak{X}_{2}$ \\
$\mathfrak{X}_{2}$ & $\mathfrak{X}_{2}$ & $\mathfrak{X}_{1}$ \\
$\mathfrak{X}_{3}$ & $\mathfrak{X}_{4}$ & - \\
$\mathfrak{X}_{4}$ & $\mathfrak{X}_{3}$ & $\mathfrak{X}_{4}$
\end{tabular}.

Let $X_{i}$ be a generator for $\mathfrak{X}_{i}$, so that we have the following table:

\begin{tabular}{c|c} 
& $I$ \\
\hline$X_{1}$ & $X_{1}^{-1}$ \\
$X_{2}$ & $X_{2}^{-1}$ \\
$X_{3}$ & $X_{3}$ \\
$X_{4}$ & $X_{4}$
\end{tabular}.

Let $\mathfrak{N}=\langle J, Q\rangle$. Since $\mathfrak{N} \subseteq \mathfrak{M}$, the structure of $\mathfrak{N}$ may be easily determined. Let $\mathfrak{Q}_{1}, \mathfrak{\Omega}_{1}^{*}$ be the quaternion subgroups of $\boldsymbol{O}_{3}(\mathfrak{M}), \mathfrak{Q}_{1}$ being as above. As $J$ normalizes $\left\langle\mathfrak{X}_{3}, \mathfrak{X}_{4}\right\rangle$ and $\left\langle\mathfrak{X}_{3}, \mathfrak{X}_{4}\right\rangle$ is a $S_{3}$-subgroup of $\mathfrak{M}$, it follows that $\mathfrak{\Omega}_{1}^{J}=\mathfrak{\Omega}_{1}^{*}$. Hence, $(J Q)^{2}=J Q J Q$ is an involution distinct from $I$. This means that $\mathfrak{N} /\langle I\rangle$ is a dihedral group of order 8 with involutory generators $J\langle I\rangle, Q\langle I\rangle$.

Finally, notice that $\mathfrak{B}=\mathfrak{P}\langle I\rangle=N(\mathfrak{P})$.

Since $\left(J X_{1}\right)^{3}=1$ and since $\left(\Omega X_{3}\right)^{3} \in\langle I\rangle$, it is straightforward to deduce from the first table that $\mathfrak{B N B}=\mathbb{S}_{1}$ is a group. We will determine the multiplication table of $\mathbb{S}_{1}$. First, we assume without 
loss of generality that $\left(Q X_{3}\right)^{3}=1$, since replacement of $Q$ by $Q^{-1}=Q I$ will achieve this if $\left(Q X_{3}\right)^{3}=I$. Since $I$ inverts $X_{1}$ and centralizes $X_{3}$, it follows easily that $I$ neither inverts nor centralizes $\left[X_{1}, X_{3}\right]$. Thus, we may choose $X_{2}, X_{4}$ as generators for $\mathfrak{X}_{2}, \mathfrak{X}_{4}$ respectively such that

$$
\left[X_{1}, X_{3}\right]=X_{2} X_{4} \text {. }
$$

By construction, $\mathfrak{X}_{4}=3=\boldsymbol{Z}(\mathfrak{P})$, so to complete the determination of $\mathfrak{B}$, we must compute $\left[X_{1}, X_{2}\right]$. Conjugation of (9.6) by $I$ yields $\left[X_{1}^{-1}, X_{3}\right]=X_{2}^{-1} X_{4}$, from which we find easily that $\left[X_{1}, X_{2}\right]=X_{4}$.

Let $X_{1}^{Q}=X_{2}^{a}$. Since $\left(Q X_{3}\right)^{3}=1$, an easy calculation (conjugation of (9.6) by $Q$ ) shows that $a=+1$. Since $J \in \mathfrak{I}_{2} \triangleleft \mathfrak{E}_{2}$, it follows that $C_{\mathfrak{O}}(J)$ has order 3 . Since $J$ normalizes but does not invert $\left\langle X_{3}, X_{4}\right\rangle$, it follows that $C_{9 \mathfrak{A}}(J) \subseteq\left\langle X_{3}, X_{4}\right\rangle$. Hence, $X_{2}^{J}=X_{2}^{-1}$. Let $X_{3}^{J}=X_{4}^{b}$. Since $\left(X_{1} J\right)^{3}=1$, an easy calculation (conjugation of (9.6) by $J$ ) shows that $b=-1$.

Set $W_{0}=\left(J Q^{2}\right)$. We argue that

$$
\mathfrak{B} \cap \mathfrak{B}^{W_{0}}=1 \text {. }
$$

Suppose by way contradiction that 9.7 is false. Since $W_{0}$ is an involution, $\mathfrak{D}=\mathfrak{P} \cap \mathfrak{P}^{W_{0}}$ is normalized by $W_{0}$. Since $W_{0} \in \boldsymbol{Z}(\mathfrak{R})$, it follows that $\mathfrak{S}=\langle I\rangle$ also normalizes $\mathfrak{D}$. Since $\boldsymbol{C}_{\mathfrak{S}}(I)=\left\langle X_{3}, X_{4}\right\rangle$ and since $W_{0} \in \mathfrak{M}$, it follows from the construction of $\mathfrak{N}$ that $I$ inverts $\mathfrak{D}$. Thus, $\mathfrak{D} \subset \boldsymbol{O}_{3}\left(\mathfrak{B}_{1}\right)$, since $\boldsymbol{O}_{3}\left(\mathfrak{B}_{1}\right)$ contains all the elements of $\mathfrak{P}$ which are inverted by $I$. As $\mathfrak{D}$ is abelian, and as $I$ centralizes $\mathfrak{X}_{4}=Z\left(O_{3}\left(\mathfrak{B}_{1}\right)\right)$, it follows that $|\mathfrak{D}|=3$. There are exactly 4 subgroups of $\boldsymbol{O}_{3}\left(\mathfrak{B}_{1}\right)$ of order 3 which are inverted by $I$; they are all of the shape $\mathfrak{X}_{2}^{Q^{*}}$ for some $Q^{*}$ in $\mathfrak{Q}_{1}$. Since $I$ normalizes $\mathfrak{X}_{2}$ and since $\mathfrak{\Omega}_{1}=\langle Q\rangle \cup\left\langle Q^{X_{3}}\right\rangle \cup\left\langle Q^{X_{3}^{-1}}\right\rangle$, we may assume that $\mathfrak{D}=\mathfrak{X}_{2}^{Q^{*}}$, where $Q^{*}$ is one of $1, Q, X_{3}^{-1} Q X_{3}, X_{3} Q X_{3}^{-1}$. Since $W_{0}$ normalizes $\mathfrak{D}$, we get that $Q^{*} W_{0} Q^{*-1} \in N\left(\mathfrak{X}_{2}\right)$. Since $Q^{*} \in \mathfrak{M}$ and $W_{0} \in \boldsymbol{O}_{2}(\mathfrak{M})$, we get that $Q^{*} W_{0} Q^{*-1} \in N\left(\mathfrak{X}_{2}\right) \cap \boldsymbol{O}_{2}(\mathfrak{M})=\mathbb{R}$, say. Since $I$ inverts $X_{2}$, it follows that $I \notin D(\&)$. Thus, $\mathcal{L}$ is elementary. But 1 and $\langle I\rangle$ are the only elementary subgroups of $\boldsymbol{O}_{2}(\mathfrak{M})$ which admit $\left\langle X_{3}, X_{4}\right\rangle$, so $Q^{*} W_{0} Q^{*-1}=\langle I\rangle$. This is not the case, since $Q^{*} \in \mathfrak{M}$, $I \in Z(\mathfrak{M})$, and $I \neq W_{0}$. This proves (9.7).

Set $\mathfrak{B}=\left\{1, Q, J, Q J, J Q, Q J Q, J Q J, W_{0}\right\}$, a set of representatives for the cosets of $\mathfrak{K}$ in $\mathfrak{R}$. For each $W$ in $\mathfrak{B}$, let $\mathfrak{B}_{W}=\left\langle\mathfrak{X}_{i}\right| 1 \leqq i \leqq 4$, $\left.\mathfrak{X}_{i}^{W-1} \subseteq \mathfrak{P}^{W_{0}}\right\rangle$. It follows that condition (iii) of Théorème I of [36] is satisfied, so by that theorem, so is condition (ii), that is, if $W_{1}, W_{2} \in \mathfrak{W}$ and $B W_{1} B=B W_{2} B$, then $W_{1}=W_{2}$. In view of our preceding information, we conclude that each element of $\mathbb{S}_{1}$ has a normal form of the shape $P H W P^{\prime}$, where $P \in \mathfrak{P}, H \in \mathfrak{S}, W \in \mathfrak{W}, P^{\prime} \in \mathfrak{B}_{W}$. Furthermore, it is clear that the normal forms for $P H W P^{\prime} J$ and $P H W P^{\prime} Q$ are determined by our information. This implies immediately that if (5)* is any group which satisfies the hypothesis of Theorem 9.1 and $\mathrm{Hy}-$ 
pothesis 9.1 , then $\mathbb{S B}^{*}$ contains a subgroup $\mathbb{B}_{1}^{*}$ isomorphic to $\mathbb{S}_{1}$. Taking (3) $=S_{4}(3)$, a comparison of orders yields $\mathscr{B S}_{1} \cong S_{4}(3)$. In particular, $i\left(\mathfrak{S}_{1}\right)=2$ and $I, W_{0}$ are representatives for the two classes of involutions of $\mathscr{S}_{1}$. Since $\mathfrak{M}=\left\langle Q_{1}, J, X_{3}, X_{4}\right\rangle$, we have $\mathfrak{M} \subseteq \mathbb{S}_{1}$. We will show that $\boldsymbol{C}\left(W_{0}\right) \subseteq \mathscr{G}_{1}$. Let $\Re=\boldsymbol{O}_{2}\left(\boldsymbol{C}_{\mathscr{G}_{1}}\left(W_{0}\right)\right)$. Then $\Re$ is elementary of order $2^{4}$ and $\Re$ is characteristic in a $S_{2}$-subgroup of $C_{\mathscr{S}_{1}}\left(W_{0}\right)$. Thus, it suffices to show that $N(\Re)=N_{\mathscr{S}_{1}}(\Re)$. Since $N_{\mathscr{S}_{1}}(\Re)$ is an extension of $\Re$ by $A_{5}$ and since $\Re=C(\Re)$, it follows that $A_{\mathscr{S}}(\Re)$ is a subgroup of Aut $(\Re)=$ $L_{4}(2)$ which contains a subgroup isomorphic to $A_{5}$ and has $S_{2}$-subgroups of order 4. Hence, $\boldsymbol{A}_{\overparen{S}}(\Re)=\boldsymbol{A}_{\mathscr{S}_{1}}(\Re) \cong A_{5}$. Hence, $\mathscr{B}_{1}$ contains the centralizer of each of its involutions. By Lemma 5.35, $\mathbb{B}=\mathbb{S}_{1}$. Thus, Theorem 9.1 is proved in case Hypothesis 9.1 is satisfied.

We now revert to our previous notation.

HYPOTHESIS 9.2. $\quad \mathfrak{S}$ is of symplectic type and width $w \geqq 2$.

Hypothesis 9.3. (i) $\mathfrak{K}$ is extra special of order $3^{5}$.

(ii) $|\mathfrak{P}|=3^{6}$.

(iii) 3 is not weakly closed in $\mathfrak{S}$.

Lemmas 9.3 through 9.10 are all proved under Hypothesis 9.2. Notice that Hypothesis 9.3 trivially implies Hypothesis 9.2.

Lemma 9.3. (i) C(3) does not contain a four-group.

(ii) If $\mathfrak{Q}$ is any abelian 2-subgroup of $\mathfrak{N}$, then $\boldsymbol{A}_{\mathfrak{N}}(\mathfrak{Q})$ is a 2group.

(iii) If $\mathfrak{X}$ is any subgroup of $\mathfrak{S E}$ of type $(3,3)$ which contains 3 , then $|\boldsymbol{C}(\mathfrak{U})|$ is odd.

(iv) If $\mathfrak{A}$ is any subgroup of $\mathfrak{S}$ of type $(3,3)$ which contains 3 , then $\mathfrak{A} \in \mathscr{E}(3)$.

Proof. Clearly, (i) implies (ii), and (iii) implies (i). Suppose (iv) holds, but $I$ is an involution in $C(\mathfrak{X})$. By Lemma 5.37, $C(I)$ contains an element of $\mathscr{C}(2)$. By Lemma 7.4, $C(I)$ is nonsolvable. Hence, (iv) implies (iii). To complete the proof of the lemma, it suffices to prove (iv). However, (iv) is a consequence of Lemma 7.2.

Lemma 9.4. Suppose $B \in \Omega_{1}(\mathfrak{S})-3$ and $\mathfrak{S}_{0}=C_{\mathfrak{5}}(B)$. Then

$$
\boldsymbol{C}\left(\mathfrak{S}_{0}\right)=\boldsymbol{Z}\left(\mathfrak{S}_{0}\right)=\langle B\rangle \times \boldsymbol{Z}(\mathfrak{\mathfrak { Y }}) .
$$

Proof. Since $3 \subset \mathfrak{F}_{0}$, it follows that $C\left(\mathfrak{S}_{0}\right)=\boldsymbol{C}_{\mathfrak{R}}\left(\mathfrak{K}_{0}\right)$. Since a $S_{3^{\prime}}$ subgroup of $\mathfrak{R}$ is faithfully represented on $\mathfrak{K}$, it follows that $C\left(\mathfrak{S}_{0}\right)$ is a 3-group. It suffices to show that $C\left(\mathfrak{K}_{0}\right) \subseteq \mathfrak{F}_{2}$. Suppose false and 
$C \in C\left(\mathscr{S}_{0}\right), C \notin \mathscr{\mathcal { F }}$. We may assume that $C^{3} \in \mathfrak{\mathcal { F }}$. In this case, $\langle C\rangle /\left\langle C^{3}\right\rangle$ is faithfully represented on $\boldsymbol{Q}_{3}^{1}(\mathfrak{N})$ and by Lemma 5.30, it follows that $\left[\boldsymbol{Q}_{3}^{1}(\mathfrak{R}),\langle C\rangle\right]=\widetilde{\mathfrak{Q}}$ is a quaternion group. Let $\mathfrak{Q}$ be a subgroup of $\mathfrak{R}$ incident with $\tilde{\mathfrak{Q}}$. Clearly, $\mathfrak{S}=C_{\mathfrak{S}}(\mathfrak{Q})[\mathfrak{\Omega}, \mathfrak{S}]$ and $\boldsymbol{C}_{\mathfrak{S}}(\mathfrak{Q})$ commutes elementwise with $[\Omega, \mathfrak{S}]$. By Lemma 9.3 (iii), $\mathfrak{\Omega}^{\prime}$ centralizes no noncyclic subgroup of $\mathfrak{S C}_{\text {. }}$ It follows that $\boldsymbol{C}_{\mathfrak{S}}(\mathfrak{Q})=\boldsymbol{Z}(\mathfrak{S})$ is cyclic. However, $w \geqq 2$ and $C$ centralizes $\mathfrak{S}_{0}$.

\section{LEMMA 9.5. Hypothesis 9.3 is not satisfied.}

\section{Proof. Suppose false.}

Let $\mathscr{Z}=\left\{Z_{1} \mid Z_{1} \subseteq \mathfrak{K}_{2}, Z_{1} \sim \mathcal{Z}, Z_{1} \neq 3\right\}$. By Hypothesis 9.3 (iii), $\mathscr{Z} \neq \varnothing$. Since $\mathfrak{K} \triangleleft \mathfrak{R}$, $\mathscr{Z}$ is invariant in $\mathfrak{R}$. Choose $\beta_{1} \in \mathscr{Z}$ such that $C_{\mathfrak{F}}\left(3_{1}\right)$ is a $S_{3}$-subgroup of $C_{\mathfrak{R}}\left(3_{1}\right)$. Let $\mathfrak{P}_{0}=C_{\mathfrak{P}}\left(3_{1}\right)$.

If $\mathfrak{P}_{0}=C_{\mathfrak{H}}\left(\mathfrak{Z}_{1}\right)$, then 3 char $\mathfrak{P}_{0}$. This is impossible since $\mathfrak{P}_{0}$ is not a $S_{3}$-subgroup of $C\left(3_{1}\right)$. Hence, $\left|\mathfrak{P}_{0}\right|=3^{5}$.

Let $\mathfrak{D}=\left\langle\mathcal{Z}, \mathfrak{Z}_{1}\right\rangle$, so that $\mathfrak{D} \subseteq \boldsymbol{Z}\left(\mathfrak{P}_{0}\right)$. If $\mathfrak{D} \subset \boldsymbol{Z}\left(\mathfrak{P}_{0}\right)$, then choose $Z \in Z\left(\mathfrak{P}_{0}\right)-\mathfrak{S}$, so that $Z$ centralizes a 3 -dimensional subspace of $\mathfrak{S} / \mathfrak{F}^{\prime}$. This implies that some involution of $\boldsymbol{O}^{3 \prime}(\mathfrak{N})$ has a noncyclic fixed point set on $\mathfrak{S}$, in violation of Lemma 9.3 (iii). Hence, $\mathfrak{D}=\boldsymbol{Z}\left(\mathfrak{P}_{0}\right)$.

Let $\mathfrak{B}^{*}$ be a $S_{3}$-subgroup of $C\left(B_{1}\right)$ which contains $\mathfrak{\beta}_{0}$. Thus, $\left\langle\mathfrak{P}, \mathfrak{P}^{*}\right\rangle \leqq N\left(\mathfrak{P}_{0}\right) \subseteq N(\mathfrak{D})$, so $O^{3^{\prime}}\left(A_{\mathscr{S}}(\mathfrak{D})\right) \cong S L(2,3)$.

By Lemma 9.3, $|C(\mathfrak{D})|$ is odd. Since $\mathfrak{P}_{0}$ is a $S_{3}$-subgroup of $C(\mathfrak{D})$, and since $O_{3^{\prime}}(C(D))=1$, it follows that $\mathfrak{P}_{0}=C(\mathfrak{D})$. Hence, $N\left(\mathfrak{P}_{0}\right)=$ $N(\mathfrak{D})$. Let $\mathfrak{M}=N(\mathfrak{D})$.

Let $\mathfrak{Q}$ be a $S_{2}$-subgroup of $\boldsymbol{O}^{\prime \prime}(\mathfrak{R})$. Thus, $\mathfrak{Q}$ is a quaternion group. Let $J$ be the involution of $\mathfrak{\Omega}$. Let $\mathfrak{\Omega}^{*}$ be a $S_{2}$-subgroup of $\boldsymbol{O}^{3^{\prime}}(\mathfrak{M})$. Thus $\Omega^{*}$ is a quaternion group. Let $I$ be the involution of $\mathfrak{R}^{*}$. Since $J$ inverts $\mathfrak{S} / \mathfrak{S}^{\prime}, J \in \mathfrak{M}$. Since $I$ inverts $\mathfrak{D}, I \in \mathfrak{R}$. We assume without loss of generality that $I$ normalizes $\cong$ and $J$ normalizes $\Omega^{*}$.

Since $J$ neither inverts nor centralizes $\mathfrak{D}$, it is clear that $\boldsymbol{A}_{\mathscr{S}}(\mathfrak{D}) \cong$ $G L(2,3)$ and so $\left\langle J, \mathfrak{\Omega}^{*}\right\rangle$ is isomorphic to a $S_{2}$-subgroup of $G L(2,3)$. Let $Q^{*}$ be an element of $\mathfrak{\Omega}^{*}$ of order 4 which is inverted by $J$.

We will show that $\langle I, \mathfrak{\Omega}\rangle$ is isomorphic to a $S_{2}$-subgroup of $G L(2,3)$. Since $I \in \mathfrak{R}$, we need only prove that $I$ inverts $\mathfrak{P}_{0} / \mathfrak{P}_{0} \cap \mathfrak{S}_{\mathrm{C}}$. Suppose false. Then $I$ centralizes $\mathfrak{P}_{0} / \mathfrak{P}_{0} \cap \mathfrak{S}_{\mathcal{C}}$. We know that $\mathfrak{D} \subseteq \mathfrak{P}_{0}^{\prime}$, because $\mathfrak{M}$ operates irreducibly on $\mathfrak{D}$ and $\mathfrak{D}$ contains $\mathbb{Z}=\left(\mathfrak{P}_{0} \cap \mathfrak{F}\right)^{\prime}$. Since $\mathfrak{Z}^{*}$ is faithfully represented on $\mathfrak{P}_{0}$, there must be a 2 -dimensional subspace of $\mathfrak{P}_{0} / \boldsymbol{D}\left(\mathfrak{F}_{0}\right)$ which $I$ inverts and $\mathfrak{\Omega}^{*}$ leaves invariant. Since $I$ centralizes $\mathfrak{P}_{0} / \mathfrak{F}_{0} \cap \mathfrak{S}$ and $\left|\mathfrak{P}_{0}\right|=3^{5}$, we conclude that $\mathfrak{D}=\mathfrak{P}_{0}^{\prime}=\boldsymbol{D}\left(\mathfrak{P}_{0}\right)$, and that $\mathfrak{P}_{0} \cap \mathfrak{F} / \mathscr{D}$ is the subspace of $\mathfrak{P}_{0} / \mathfrak{P}_{0}^{\prime}$ inverted by $I$. This forces $I$ to invert both $\mathfrak{P}_{0} \cap \mathfrak{S}_{2} / \mathcal{D}$ and $\mathfrak{D}$, that is, to invert $\mathfrak{P}_{0} \cap \mathfrak{S}_{\mathrm{S}}$. So $\mathfrak{P}_{0} \cap \mathfrak{S}_{\mathcal{C}}$ is abelian, which is false.

Let $Q$ be an element of $\cong$ of order 4 which is inverted by $I$. 
Set $\mathfrak{X}_{6}=3$. Since $J$ normalizes $\mathfrak{D}$ and centralizes $\mathfrak{X}_{6}$, we can choose an element $X_{5}$ of $\mathfrak{D}$ of order 3 such that $X_{5}^{J}=X_{5}^{-1}$. Let $\mathfrak{X}_{5}=$ $\left\langle X_{5}\right\rangle, \mathfrak{X}_{4}=\mathfrak{X}_{5}^{Q}, X_{4}=X_{5}^{Q}$. Then we have relations $X_{5}^{I}=X_{5}^{-1}, X_{5}^{J}=X_{5}^{-1}$, $X_{4}^{I}=X_{4}, X_{4}^{J}=\mathfrak{X}_{4}^{-1}$. Suppose $\left[X_{4}, X_{5}\right] \neq 1$. The following argument is designed to exclude this possibility.

Let $\left[X_{4}, X_{5}\right]=X_{6}$ so that $X_{6}$ is a generator for $\mathfrak{X}_{6}$. Since $X_{4} \notin \mathfrak{P}_{0}$, it follows that $\mathfrak{P}_{0} \cap \boldsymbol{C}(I)$ is of order 3 with generator $X_{3}$, say. Thus, $\left[\left\langle X_{3}, X_{4}\right\rangle=C_{\mathfrak{P}}(I)\right.$ is of order 9 , so that $\left[X_{3}, X_{4}\right]=1$. As $\mathfrak{S}$ contains all the elements of $\mathfrak{P}$ which are centralized by $I$, we have $X_{3} \in H$. Since $\left\langle X_{3}\right\rangle=$ $\boldsymbol{C}_{\mathfrak{B}_{0}}(I)$, it follows that $J$ normalizes $\left\langle X_{3}\right\rangle$, so that $X_{3}^{J}=X_{3}^{-1}$, as $J$ inverts $\mathfrak{S} / 3$. Let $X_{2}=X_{3}^{Q}$. Since $\left[X_{3}, X_{4}\right]=1$, so also $\left[X_{2}, X_{4}^{Q}\right]=1$. But $X_{4}^{Q}=$ $X_{5}^{Q^{2}}=X_{5}^{-1}$, so $X_{2}$ centralizes $X_{5}$, that is, $X_{2} \in \mathfrak{P}_{0}$. Let $X_{1}=X_{2}^{Q^{*}}$, and let $\mathfrak{X}_{i}=\left\langle\mathfrak{X}_{i}\right\rangle, 1 \leqq i \leqq 6$. We obtain the following data:

Table 1

\begin{tabular}{c|l|l} 
& \multicolumn{1}{|c|}{$J$} & \multicolumn{1}{|c}{$I$} \\
\hline$X_{1}$ & $X_{1}$ & $X_{1}^{-1}$ \\
$X_{2}$ & $X_{2}^{-1}$ & $X_{2}^{-1}$ \\
$X_{3}$ & $X_{3}^{-1}$ & $X_{3}$ \\
$X_{4}$ & $X_{4}^{-1}$ & $X_{4}$ \\
$X_{5}$ & $X_{5}^{-1}$ & $X_{5}^{-1}$ \\
$X_{6}$ & $X_{6}$ & $X_{6}^{-1}$
\end{tabular}

Table 2

\begin{tabular}{l|l|l} 
& \multicolumn{1}{|c|}{$Q$} & \multicolumn{1}{|c}{$Q^{*}$} \\
\hline$X_{1}$ & - & $X_{2}^{-1}$ \\
$X_{2}$ & $X_{3}^{-1}$ & $X_{1}$ \\
$X_{3}$ & $X_{2}$ & $X_{3}$ \\
$X_{4}$ & $X_{5}^{-1}$ & - \\
$X_{5}$ & $X_{4}$ & $X_{6}^{-a}$ \\
$X_{6}$ & $X_{6}$ & $X_{5}^{a}$
\end{tabular}

Here $a^{2}=1$, and the last two entries in Table 2 are at our disposal since $Q^{*}$ normalizes $\langle I, J\rangle$ and since $\mathfrak{X}_{5}, \mathfrak{X}_{6}$ are the only subgroups of (D) of order 3 which admit $\langle I, J\rangle$. In addition we have the following: commutation relations:

$$
\begin{aligned}
& {\left[X_{i}, X_{6}\right]=1,1 \leqq i \leqq 6,\left[X_{4}, X_{5}\right]=X_{6},} \\
& {\left[X_{i}, X_{5}\right]=1,1 \leqq i \leqq 3,\left[X_{3}, X_{4}\right]=\left[X_{2}, X_{4}\right]=1 .}
\end{aligned}
$$

Furthermore, $\left[X_{2}, X_{3}\right]=X_{6}^{b}$, so by Table 2 , we get $\left[X_{1}, X_{3}\right]=X_{5}^{a b}$. Here $b^{2}=1$, for if $b=0$, we get $X_{3} \in Z(\mathfrak{F})$, which is not the case. The as yet undetermined commutation relations are:

$$
\left[X_{1}, X_{4}\right]=X_{2}^{x} X_{3}^{y} X_{5}^{z} X_{6}^{t}, \quad\left[X_{1}, X_{2}\right]=X_{3}^{c} X_{5}^{d} X_{6}^{e} .
$$

Use Table 1 and conjugate the second relation by $J$, obtaining $e=b c$. Then conjugation by $I$ yields $d=a b c$. Conjugation of the first relation by $J$ yields $t=x y b+z$. Conjugation of the first relation by $I$ yields $y=c x$.

Assume $c \neq 0$. Then

$$
\mathfrak{P}_{0}^{\prime}=\left\langle X_{3}, X_{5}, X_{6}\right\rangle,\left[\mathfrak{P}_{0}^{\prime}, \mathfrak{P}_{0}\right]=\left\langle X_{5}, X_{6}\right\rangle=\mathfrak{D}=\boldsymbol{Z}\left(\mathfrak{P}_{0}\right) \text {. }
$$

We see that $\mathfrak{P}_{0}^{\prime}$ is elementary abelian. If $A \in \mathfrak{P}_{0}, B \in \mathfrak{P}_{0}^{\prime}$, then $(A B)^{3}=$ $A^{3} B^{A^{2}+A+1}$. But $c l\left(\mathfrak{P}_{0}\right)=3$ and so $B^{A^{2}+A+1}=B^{3}[B, A]^{3}=1$. Hence, 
there is a map $\varphi$ of $\mathfrak{P}_{0} / \mathfrak{P}_{0}^{\prime}$ given by $\varphi\left(A \mathfrak{P}_{0}^{\prime}\right)=A^{3}$.

Clearly, $\varphi\left(X_{1} \mathfrak{P}_{0}^{\prime}\right)=1$. But $\mathfrak{M}$ operates as $G L(2,3)$ on $\mathfrak{D}$. Since $\left|\mathfrak{P}_{0}: \mathfrak{P}_{0}^{\prime}\right|=3^{2}$, this forces $\mathfrak{M}$ to operate as $G L(2,3)$ on $\mathfrak{P}_{0} / \mathfrak{P}_{0}^{\prime}$. In particular, the four subgroups of $\mathfrak{P}_{0} / \mathfrak{P}_{0}^{\prime}$ of order 3 are all conjugate under $\mathfrak{M}$. Hence, $\varphi\left(A \Re_{0}^{\prime}\right)=1$ for all $A$ in $\mathfrak{P}_{0}$ and $\mathfrak{B}_{0}$ is of exponent 3 .

By [21, p. 324], the order of the Burnside group of exponent 3 on 2 generators is 27. Since $\mathfrak{P}_{0}$ must be a homomorphic image of this group, we get a contradiction, as $\left|\mathfrak{P}_{0}\right|=3^{5}>27$. So $c=0$.

Since $c=0$, so also $c=d=e=y=0$. Since $y=0$, we also have $t=z$. Conjugation of the first relation by $Q$ yields $\left[Q^{-1} X_{1} Q, X_{5}^{-1}\right]=$ $X_{3}^{-x} X_{4}^{z} X_{6}^{z}$. Now $C(J) \cap \boldsymbol{O}^{3^{\prime}}(\mathfrak{R})=\left\langle X_{1}, X_{6}, \mathfrak{Q}\right\rangle$, so $\boldsymbol{C}(J) \cap \boldsymbol{O}^{3^{\prime}}(\mathfrak{R})$ is 2-closed, that is, $X_{1}$ normalizes $\supseteq$. Hence, $\left(Q X_{1}\right)^{3}=J^{u}, u=0$ or 1 . Hence, $Q^{-1} X_{1} Q=J X_{1}^{-1} Q^{-1} X_{1}^{-1} J^{u}$. The previous commutation relation now yields $x=0$.

Since $x=0$, it follows that $X_{4}$ centralizes $\mathfrak{P}_{0} / \mathfrak{D}$. Hence, $\mathfrak{Q}^{*}$ is forced to centralize $\mathfrak{P}_{0} / \mathfrak{D}$. This is not the case, since $\mathfrak{D} \subseteq \mathfrak{P}_{0}^{\prime}$. We conclude that $\left[X_{4}, X_{5}\right]=1$.

Since $I$ centralizes $X_{4}$, it follows that $\left\langle X_{4}, X_{5},, X_{6}\right\rangle=\mathbb{5} \triangleleft \mathfrak{M}$. Namely, $\mathfrak{D} \triangleleft \mathfrak{M}$, so we need to show that $\mathfrak{F} / \mathfrak{D} \triangleleft \mathfrak{M} / \mathcal{D}$. Since $X_{4}$ centralizes $\mathfrak{D}$, we have $X_{4} \in \mathfrak{P}_{0}$. Since $\mathfrak{P}_{0} / \mathfrak{D}$ is of order 27 and admits $\mathfrak{Q}^{*}$ as a group of automorphisms, it follows that $\mathfrak{E} / \mathfrak{D}=C_{\mathfrak{P}_{0} / \mathfrak{D}}(I) \triangleleft \mathfrak{M} / \mathfrak{D}$. Thus, $\langle\mathfrak{M}, Q\rangle \subseteq N(\mathfrak{S})$. Since $\langle\mathfrak{P}, Q\rangle=O^{3^{\prime}}(\mathfrak{R})$, it follows that both $\mathfrak{M}$ and $O^{3^{\prime}}(\mathfrak{R})$ are subgroups of $N(\mathfrak{F})$.

Let $\mathfrak{F}^{*}=O_{3}(N(\mathfrak{F}))$. Thus,

$$
\mathfrak{F} \subseteq \mathfrak{F}^{*} \subseteq \boldsymbol{O}_{3}(\mathfrak{M}) \cap \boldsymbol{O}_{3}(\mathfrak{R})=\mathfrak{P}_{0} \cap \mathfrak{S} \text {. }
$$

Suppose $\mathfrak{F}^{*}=\mathfrak{P}_{0} \cap \mathfrak{S}$. Then $3=\mathfrak{F}^{* \prime} \triangleleft N(\mathfrak{F})$, against $\boldsymbol{O}^{3^{\prime}}(\mathfrak{R}) \subset \boldsymbol{N}(\mathfrak{F})$. Hence, $\quad \mathfrak{F}^{*} \subset \mathfrak{P}_{0} \cap \mathfrak{F}$. Since $|\mathfrak{F}|=3^{3}$ and $\left|\mathfrak{P}_{0} \cap \mathfrak{S}\right|=3^{4}$, it follows that

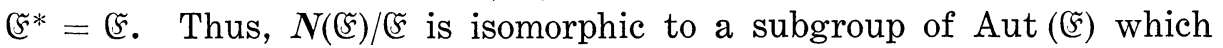
(a) is solvable, (b) contains a $S_{3}$-subgroup of Aut(卢), (c) is 3-reduced. There are no such groups. The proof of the lemma is complete.

Lemma 9.6. Let $\mathfrak{B}$ be a subgroup of $\mathfrak{S}$ of type $(3,3)$. Then $\mathfrak{B} \in \mathscr{D}$. (See Definition 7.3.)

Proof. We first show that if $B \in \mathfrak{B}$, then

(9.8) for some $N$ in $\mathfrak{R}, B$ centralizes an element of $\mathscr{C}\left(\mathfrak{P}^{N}\right)$.

Let

$$
\mathfrak{S}_{1}=\Omega_{1}(\mathfrak{S}), \mathfrak{B}=\mathfrak{S}_{1} / D\left(\mathfrak{S}_{1}\right), \mathfrak{B}_{0}=\boldsymbol{C}_{\mathfrak{B}}(\mathfrak{P})
$$

Suppose $\left|\mathfrak{B}_{0}\right|>3$. Then $\mathfrak{B}_{0}=\mathfrak{W} / \boldsymbol{D}\left(\mathfrak{F}_{1}\right)$ and every subgroup of $\mathfrak{W}$ which contains $\boldsymbol{D}\left(\mathfrak{S}_{1}\right)$ is normal in $\mathfrak{P}$. Let $\mathfrak{W}_{1}=\mathfrak{W} \cap \boldsymbol{C}(B)$ so that $\left|\mathfrak{W}: \mathfrak{W}_{1}\right| \leqq 3$. 
Since $\left|\mathfrak{B}_{0}\right|>3$, so also $\left|\mathfrak{W}_{1}\right| \geqq 9$. Thus, $B$ centralizes an element of $\mathscr{U}(\mathfrak{B})$ in this case. We may assume that $\left|\mathfrak{V}_{0}\right|=3$.

Suppose $\mathfrak{N} / \mathfrak{S}$ has a normal subgroup $\mathfrak{R} / \mathfrak{F}=\mathfrak{X}$ of odd order $\neq 1$. Let $k$ be a field of characteristic 3 which contains all $|\mathfrak{X}|^{\text {th }}$ roots of 1 . Let $\widetilde{\mathfrak{B}}=k \bigotimes_{F_{3}} \mathfrak{B}$. Thus, $\widetilde{\mathfrak{B}}$ admits $\mathfrak{N} / \mathfrak{S}$ and $k \otimes \mathfrak{B}_{0}$ is the set of all fixed points of $\mathfrak{P} / \mathfrak{S}$ on $\widetilde{\mathfrak{B}}$. Let $\widetilde{\mathfrak{B}}=\bigoplus_{\rho} \widetilde{\mathfrak{B}}(\rho)$, where $\widetilde{\mathfrak{B}}(\rho)$ is the largest $\mathfrak{X}$-submodule of $\widetilde{\mathfrak{B}}$ on which $\mathfrak{X}$ acts as a multiple of the irreducible representation $\rho$. Since $\widetilde{\mathfrak{B}}$ inherits the non singular symplectic structure of $\mathfrak{B}$, it follows that $\rho$ and $\rho^{*}$ appear with the same multiplicity in $\widetilde{\mathfrak{B}}, \rho^{*}$ denoting the contragredient representation of $\rho$. Since $|\mathfrak{P}|$ is odd, $\widetilde{\mathfrak{B}}(\rho)$ and $\widetilde{\mathfrak{B}}\left(\rho^{*}\right)$ are not conjugate under $\mathfrak{P}$. Hence, $\mathfrak{B}_{0}$ is not 1 dimensional in this case.

We may now assume that

$$
\boldsymbol{F}(\mathfrak{N} / \mathfrak{S}) \text { is a } 2 \text {-group . }
$$

If $\mathfrak{K}=\mathfrak{P}$, then (9.8) is obvious, so suppose $\mathfrak{S} \subset \mathfrak{P}$. Set $\mathfrak{R}^{*}=C(3)$, so that $\left|\mathfrak{R}: \mathfrak{N}^{*}\right| \leqq 2$. By Lemma 9.3 (i), together with (9.9), we conclude that $\mathfrak{N}$ is a 2,3-group, and that a $S_{2}$ subgroup of $\mathfrak{R}^{*}$ is quaternion. Hence, $|\mathfrak{S}: \mathfrak{S}|=3$. Since $\left|\mathfrak{B}_{0}\right|=3$, we get that the width of $\mathfrak{S}$ is 1 , against Hypothesis 9.2. Thus, (9.8) holds.

Suppose $\mathfrak{B} \notin \mathscr{D}$. Then $И(\mathfrak{B} ; 2)$ contains a four-group $\mathfrak{Q}$ which is not centralized by $\mathfrak{B}$. Hence, $[\mathfrak{B}, \mathfrak{Q}]=\mathfrak{Q}$, and $\mathfrak{B}_{0}=\boldsymbol{C}_{\mathfrak{B}}(\mathfrak{Q})$ is of order 3 .

Let $\mathfrak{C}=\boldsymbol{C}\left(\mathfrak{B}_{0}\right), \mathfrak{S}_{0}=\boldsymbol{C}_{\mathfrak{S}}\left(\mathfrak{B}_{0}\right)$. By Lemma 7.2 applied to $\left\langle\mathfrak{B}_{0}, 3\right\rangle$, it follows that 3 centralizes $\boldsymbol{O}_{3^{\prime}}(\mathfrak{C})$. Hence, $\left[\boldsymbol{O}_{3^{\prime}}(\mathfrak{C}), \mathfrak{F}_{\mathbb{C}}\right] \subseteq \mathfrak{F}_{\mathcal{C}} \cap \boldsymbol{O}_{3^{\prime}}(\mathfrak{C})=1$. This implies that $\boldsymbol{O}_{3^{\prime}}(\mathfrak{S})=1$ by Lemma 9.4.

Let $\mathfrak{P}_{0}$ be a $S_{3}$-subgroup of $\mathbb{C}$ containing $\mathfrak{S}_{0}$ and let $\mathfrak{P}^{*}$ be a $S_{3}$ subgroup of $\mathbb{S}$ containing $\mathfrak{P}_{0}$. Then $\mathfrak{P}^{*}=\mathfrak{P}^{G}$, so that with $3^{*}=3^{G}$, it follows that $3^{*} \cong \boldsymbol{Z}\left(\boldsymbol{O}_{3}(\mathfrak{E})\right)$. Let $\mathfrak{W}=3^{* \mathfrak{E}}$, so that $\mathfrak{W}$ is 3 -reducible in $\mathfrak{c}$. Set $\mathfrak{S}_{1}=\boldsymbol{C}_{\mathfrak{G}}(\mathfrak{W})$. We argue that $\mathfrak{S}_{1} \cap \mathfrak{Q}=1$. If not, then $\mathfrak{C} \subseteq \boldsymbol{C}(\mathfrak{W})$, as $\mathfrak{Q}$ is an irreducible $\mathfrak{B}$-module. Hence, $\mathfrak{\varrho} \subseteq \boldsymbol{C}\left(3^{*}\right)$, against Lemma 9.3 (i). Hence,

$$
\mathfrak{S}_{1} \cap \mathfrak{Q}=1
$$

By $(B)$, elements of $\mathfrak{B}-\mathfrak{B}_{0}$ have minimal polynomial $(x-1)^{3}$ on $\mathfrak{W}$.

We next argue that $3 \subseteq \mathfrak{C}_{1}$. If not, then $\mathfrak{F}_{0}$ contains an extra special subgroup of width $w-1$ disjoint from $\mathfrak{F}_{1}$. We get that $m(\mathfrak{W}) \geqq 2 \cdot 3^{w-1}$. Since $m\left(\mathfrak{W} \cap \mathfrak{S C}^{G}\right) \leqq w+1$, we have $m\left(\mathfrak{W} / \mathfrak{W} \cap \mathfrak{S C}^{G}\right) \geqq 2 \cdot 3^{w-1}-w-1$. By [32], it follows that $w^{2} \geqq 2 \cdot 3^{w-1}-w-1$. This is false for $w \geqq 3$, so $w=2$. Thus, $C(3) / \mathscr{S}$ is isomorphic to a subgroup of $G L(4,3)$ which (a) is solvable, (b) is 3-reduced, (c) has an elementary subgroup of order 27. There are no such groups. We conclude that $3 \subseteq \mathfrak{\Im}_{1}$.

Since $3 \subseteq \mathfrak{F}_{1}$, we have $\mathfrak{W} \subseteq \mathfrak{R}$, so that $[\mathfrak{W}, \mathfrak{B}, \mathfrak{B}] \subseteq \mathfrak{Z}$. Hence by $(B)$, 
Since $\mathfrak{W} \subseteq \boldsymbol{Z}\left(\mathfrak{\complement}_{1}\right)$, we get

$$
3 \subseteq \boldsymbol{Z}\left(\mathfrak{\complement}_{1}\right)
$$

which implies that

$$
\mathfrak{\mho}_{1} \subseteq \mathfrak{N}
$$

By (9.13), we get $\left[\mathfrak{\complement}_{1}, \mathfrak{B}, \mathfrak{B}\right] \subseteq 3$, and in particular,

$$
\left[\mathrm{O}_{3}(\mathfrak{C}), \mathfrak{B}, \mathfrak{B}\right]=3,
$$

equality holding by (9.11) and the obvious containment $\mathfrak{W} \subseteq O_{3}(\mathfrak{S})$

Now (9.14) and (9.11) yield

$$
\boldsymbol{O}_{3}(\mathfrak{c})=\mathfrak{W}_{1} \times \mathfrak{W}_{2},
$$

where

$$
\begin{gathered}
3 \subset \mathfrak{W}_{1}=\left[\mathfrak{Q}, \boldsymbol{O}_{3}(\mathfrak{S})\right], \text { and } \mathfrak{W}_{1} \text { is elementary of order } 27, \\
\mathfrak{W}_{2}=C(\mathfrak{D}) \cap \boldsymbol{O}_{3}(\mathfrak{S}) .
\end{gathered}
$$

Let $\mathfrak{V}_{2}=\boldsymbol{O}_{3^{\prime}}\left(\mathfrak{C} \bmod \mathfrak{S}_{1}\right), \mathfrak{V}_{3}=\mathfrak{V}_{2} \boldsymbol{Z}(\mathfrak{F})$. Thus, $\mathfrak{F}_{1} \boldsymbol{Z}(\mathfrak{S})$ contains a $S_{3^{-}}$ subgroup of $\mathfrak{C}_{3}$. Thus, $\boldsymbol{Z}(\mathfrak{F})$ is normal in a $S_{3}$-subgroup of $\mathfrak{E}_{3}$. By Lemma 5.22, we get $\boldsymbol{Z}(\mathfrak{S}) \subseteq \boldsymbol{O}_{3}\left(\mathfrak{S}_{3}\right)$. Hence, $\boldsymbol{Z}(\mathfrak{S}) \subseteq \boldsymbol{O}_{3}(\mathfrak{E})$. From (9.16), we conclude that $Z(\mathfrak{S})=3$, that is,

$$
\mathfrak{S} \text { is extra special . }
$$

We argue that $\boldsymbol{O}_{3,3^{\prime}}(\mathfrak{E})$ does not centralize $\boldsymbol{Z}\left(\boldsymbol{O}_{3}(\mathfrak{S})\right)$. If it does, then since $3 \subseteq \boldsymbol{Z}\left(\boldsymbol{O}_{3}(\mathfrak{S})\right)$, it follows that $\boldsymbol{O}_{3,3^{\prime}}(\mathfrak{S}) \subseteq \mathfrak{N}$, so $\left[\boldsymbol{O}_{3,3^{\prime}}(\mathfrak{E}), \mathfrak{F}_{0}\right] \subseteq \mathfrak{F}$, which implies that $\mathfrak{S}_{0} \subseteq O_{3}(\mathfrak{C})$, which in turn gives $\mathfrak{\Omega}=[\mathfrak{B}, \mathfrak{l}] \subseteq O_{3}(\mathbb{E})$. Since $\mathfrak{B}_{0} \subseteq Z(\mathfrak{C})$, it follows that

$$
\left[\boldsymbol{Z}\left(\boldsymbol{O}_{3}(\mathfrak{S})\right), \boldsymbol{O}_{3,3^{\prime}}(\mathfrak{S})\right] \text { and } \boldsymbol{C}\left(\boldsymbol{O}_{3,3^{\prime}}(\mathfrak{S})\right) \cap \boldsymbol{Z}\left(\boldsymbol{O}_{3}(\mathfrak{S})\right)
$$

are disjoint nontrivial normal abelian subgroups of $\sqrt{5}$. In particular, if $\mathfrak{P}_{0}$ is a $S_{3}$-subgroup of $\mathfrak{c}$ containing $\mathfrak{S}_{0}$, then $\boldsymbol{Z}\left(\mathfrak{P}_{0}\right)$ is not cyclic. By Lemma 9.4 , we get that $\Omega_{1}\left(\boldsymbol{Z}\left(\mathfrak{P}_{0}\right)\right)=\mathfrak{B}_{0} \times 3$, and in particular, $\mathfrak{P}_{0} \subseteq \mathfrak{N}$.

Since $\mathfrak{P}_{0} \subseteq \mathfrak{N}$, we get that $\left[\mathfrak{P}_{0}, \mathfrak{B}, \mathfrak{B}\right] \subseteq \mathbb{3}$. Thus, if $\mathrm{B} \in \mathfrak{B}$, the minimal polynomial of $B$ on the Frattini quotient group of $\boldsymbol{O}_{3,3^{\prime}, 3}(\mathfrak{5}) /$ $\boldsymbol{O}_{3,3^{\prime}}(\mathfrak{S})$ divides $(x-1)^{2}$. By $(B)$, it follows that $\Omega$ centralizes $\boldsymbol{O}_{3,3^{\prime}, 3}(\mathfrak{S}) /$ $\boldsymbol{O}_{3,3^{\prime}}(\mathfrak{C})$, and so $\mathfrak{O} \subseteq \boldsymbol{O}_{3,3^{\prime}}(\mathfrak{C})$.

Let $\Re=\left\langle\Omega, \mathfrak{S}_{0}\right\rangle \leqq \mathbb{E}$, let $\Re_{0}=C_{\mathscr{\Re}}\left(O_{3}(\mathfrak{E})\right)$ and for any subset $\mathfrak{S}$ of $\Re$, let $\bar{\varsigma}=\subseteq \Re_{0} / \Re_{0}$.

We argue that $\overline{\mathfrak{Q}} \subseteq \boldsymbol{O}_{3^{\prime}}(\bar{\Re})$. Namely, $\mathfrak{\Omega} \subseteq \boldsymbol{O}_{3,3^{\prime}}(\mathfrak{\complement})$, and so $\mathfrak{\varrho} \subseteq \boldsymbol{O}_{3,3^{\prime}}(\Re)$, 
Thus, it suffices to show that $\left[\boldsymbol{O}_{3}(\Re), \mathfrak{Q}\right] \subseteq \Re_{0}$. But

$$
\left[O_{3}(\Re), \mathfrak{\bigcap}\right] \subseteq O_{3}(\Re) \cap O_{3,3^{\prime}}(\mathfrak{S}) \subseteq O_{3}(\Re) \cap O_{3}(\mathfrak{S}),
$$

and so

$$
\begin{aligned}
{\left[O_{3}(\Re), \mathfrak{\Omega}\right] } & =\left[O_{3}(\Re), \mathfrak{\Omega}, \mathfrak{\Omega} \subseteq\left[O_{3}(\Re) \cap O_{3}(\mathfrak{S}), \mathfrak{\bigcap}\right] \subseteq\left[O_{3}(\mathfrak{S}), \mathfrak{Q}\right]\right. \\
& =\mathfrak{W}_{1} \subseteq Z\left(O_{3}(\mathfrak{S})\right),
\end{aligned}
$$

whence $\left[\boldsymbol{O}_{3}(\Re), \mathfrak{\Omega}\right] \subseteq \Re \cap \boldsymbol{Z}\left(\boldsymbol{O}_{3}(\mathfrak{S})\right) \subseteq \Omega_{0}$.

Case 1. $\left\langle\overline{\mathfrak{\Omega}, \mathfrak{\Omega}^{H}}\right\rangle$ is abelian for all $H \in \mathfrak{S}_{0}$.

Since $\left[\overline{\mathfrak{S}^{\mathfrak{H}_{0}}, \mathfrak{B}}\right]$ admits the abelian group $\overline{\mathfrak{S}}_{0}$, and since $\mathfrak{\varrho}\left[\mathfrak{D}^{\mathfrak{S}_{0}}, \mathfrak{B}\right]$, it follows that $\left[\overline{\mathfrak{D}^{\mathfrak{L}_{0}}, \mathfrak{B}}\right]=\overline{\mathfrak{\Omega}^{\mathfrak{K}_{0}}}$. Since $\mathfrak{W}_{2}=\boldsymbol{C}(\mathfrak{Q}) \cap \boldsymbol{O}_{3}(\mathfrak{S})$ admits the abelian group $\overline{\mathfrak{Q}^{\mathfrak{W}_{0}}},(B)$ implies that $\overline{\mathfrak{\Omega}^{\mathfrak{S}_{0}}}$ centralizes $\mathfrak{W}_{2}$. Hence, $\overline{\mathfrak{D}^{\mathfrak{W}_{0}}}$ is isomorphic to an elementary 2-subgroup of Aut $\left(\mathfrak{W}_{1}\right)$. Since $\left[\overline{\mathfrak{D}^{\mathfrak{S}_{0}}, \mathfrak{B}}\right]=$ $\overline{\mathfrak{\Omega}^{\mathfrak{K}_{0}}}$, we get that $\overline{\mathfrak{D}}=\overline{\mathfrak{\Omega}^{\mathfrak{S}_{0}}}$, so that $\sqrt{\mathfrak{D}}$ is a $S_{2}$-subgroup of $\bar{\Re}$.

Let $\Re_{1}=\boldsymbol{O}_{3}\left(\Re \bmod \Re_{0}\right)$. Thus, $\Re_{1} \cap \mathfrak{S}_{0}$ is of index 3 in $\mathscr{S}_{0}$ and $\left\langle\Re_{1} \cap \mathfrak{S}_{0}, \mathfrak{B}\right\rangle=\mathfrak{S}_{0}$. Since $\left|\Re_{0}\right|$ is odd, it follows that $\mathfrak{Q}$ is a $S_{2}$-subgroup of $\Re$. Let $\mathbb{Z}=\Re_{1} O_{3}(\mathbb{C})$ and let $\mathfrak{\Omega}_{3}$ be a $S_{3}$-subgroup of $\mathbb{R}$ which contains $\Re_{1} \cap \mathscr{S}_{0}$ and is normalized by $\mathfrak{S}_{0}$. Since $|\mathbb{R}|$ is odd, it follows that $S_{2}$ subgroups of $N\left(\Omega_{3}\right) \cap \Omega \Re$ are four-groups. If $3 \subseteq D\left(\Omega_{3}\right)$, then by $(B)$, $S_{2}$-subgroups of $N\left(\Omega_{3}\right) \cap \Omega \Re$ centralize $\Omega_{3}$. This is not the case, as $\Omega$ does not centralize $\mathfrak{S}_{1}$. Hence, $3 \nsubseteq \boldsymbol{D}\left(\mathbb{R}_{3}\right)$. In particular, $3 \nsubseteq \boldsymbol{D}\left(\Re_{1} \cap \mathfrak{S}_{0}\right)$. But $\mathfrak{K}_{1} \cap \mathfrak{S}_{0}$ is of index 3 in $\mathfrak{K}_{0}$. Since $\mathfrak{F}_{\mathcal{C}}$ is extra special, it follows that $w=2$. Clearly, $\mathfrak{S} \subset \mathfrak{F}$, since $\boldsymbol{O}_{3}(\mathfrak{E})$ contains an elementary subgroup of order $3^{4}$. On the other hand, Lemma 9.3 implies that $|\mathfrak{B}: \mathfrak{S}| \leqq 3$. Hence, $|\mathfrak{B}: \mathfrak{K}|=3$, and $|\mathfrak{P}|=3^{6}$. Since $\mathfrak{B}_{0}$ is obviously not conjugate to 3 , it follows that $\boldsymbol{O}_{3}(\mathfrak{C})$ is elementary of order $3^{4}$ and $\mathfrak{P}_{0}=\boldsymbol{O}_{3}(\mathfrak{C}) \mathfrak{S}_{0}$, $\left|\mathfrak{S}_{0} \cap \boldsymbol{O}_{3}(\mathfrak{S})\right|=27$. Clearly, $\boldsymbol{O}_{3}(\mathfrak{S})$ char $\mathfrak{B}_{0}$, since $\boldsymbol{O}_{3}(\mathfrak{S})$ is the only elementary subgroup of its order in $\mathfrak{P}_{0}$.

Let $\mathfrak{M}=N\left(O_{3}(\mathbb{C})\right)$ so that $\mathfrak{M}$ contains a $S_{3}$-subgroup $\tilde{\mathfrak{P}}$ of $\mathbb{B}$ with $\tilde{\mathfrak{R}} \supset \mathfrak{P}_{0}$. Since $3=Z\left(\mathfrak{P}_{0}\right) \cap \mathfrak{P}_{0}^{\prime}$ char $\mathfrak{P}_{0}$, we have $3 \triangleleft \tilde{\mathfrak{R}}$. In particular, $\mathfrak{S C} \subset \mathfrak{M}$. We therefore assume without loss of generality that $\mathfrak{P}=\tilde{\mathfrak{P}}$.

It is clear that $\boldsymbol{O}_{3}(\mathfrak{S})=\boldsymbol{O}_{3}(\mathfrak{M})$ and that $\mathfrak{M}$ is a 2,3-group. It is equally clear that $l_{3}(\mathfrak{M})=2$, so that $\mathfrak{Q} \subseteq \boldsymbol{O}_{3,2}(\mathfrak{M})$. Hence, $\mathfrak{B}$ is a $S_{3}$ subgroup of $N(\mathfrak{Q}) \cap \mathfrak{M}$, so we can choose a subgroup $\mathfrak{B}_{1}$ of $\mathfrak{B}$ of order 3 such that $\mathfrak{B}=\mathfrak{B}_{0} \times \mathfrak{B}_{1}$ and such that $\mathfrak{B}_{1}$ normalizes some $S_{2}$-subgroup $\mathfrak{I}_{0}$ of $\boldsymbol{O}_{3,2}(\mathfrak{M})$. Let $\mathfrak{A}=\boldsymbol{N}\left(\mathfrak{I}_{0}\right) \cap \mathfrak{P}$. Thus, $\mathfrak{U}$ is elementary of order 9 , $\mathfrak{B}_{1} \subset \mathfrak{U} \quad$ and $\mathfrak{P}=\mathfrak{U} \boldsymbol{O}_{3}(\mathfrak{M}), \mathfrak{U} \cap \boldsymbol{O}_{3}(\mathfrak{M})=1$. Since $\boldsymbol{O}_{3}(\mathfrak{M}) \cap C\left(\mathfrak{B}_{1}\right)$ is of order 9 , it follows that $\boldsymbol{C}\left(\mathfrak{B}_{1}\right) \cap \mathfrak{P}$ is of order $3^{4}$. Hence, $\boldsymbol{C}\left(\mathfrak{B}_{1}\right) \cap \mathfrak{P}=$ $\boldsymbol{C}\left(\mathfrak{B}_{1}\right) \cap \mathfrak{F}$; since $\mathfrak{A}$ is elementary we get $\mathfrak{U} \subset \mathfrak{K}$.

We now choose $\mathfrak{U}_{1}$ of order 3 in $\mathfrak{U}$ so that $\mathfrak{U}$ does not centralize $\boldsymbol{C}_{\mathfrak{I}_{0}}\left(\mathfrak{H}_{1}\right)$. Let $\mathfrak{I}_{1}=\left[\boldsymbol{C}_{\mathfrak{I}_{0}}\left(\mathfrak{R}_{1}\right), \mathfrak{X}\right]$. Thus, $\mathfrak{I}_{1}$ is faithfully represented on $\boldsymbol{C}\left(\mathfrak{U}_{1}\right) \cap O_{3}(\mathfrak{M})=\Re$. It is straightforward to verify that $|\Re|=9$ and 
that $\mathfrak{I}_{1}$ is a quaternion group. Hence, $\Re=\left[\boldsymbol{O}_{3}(\mathfrak{M}), \mathfrak{N}_{1}\right]$, so $\mathfrak{R} \cong \mathfrak{L}$.

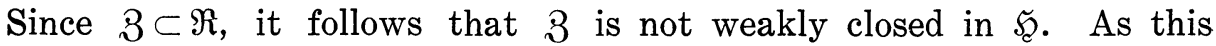
violates Lemma 9.5, we conclude that Case 1 does not hold.

Case 2. There is an element $H$ of $\mathfrak{S}_{0}$ such that $\left\langle\overline{\mathfrak{D}, \mathfrak{\Omega}^{H}}\right\rangle$ is nonabelian.

Set $\widetilde{\mathfrak{W}}=\left\langle\mathfrak{W}_{1}, \mathfrak{W}_{1}^{H}\right\rangle$, so that $\left\langle\mathfrak{Q}, \mathfrak{Q}^{H}\right\rangle$ normalizes $\widetilde{\mathfrak{W}}$ and centralizes $\boldsymbol{O}_{3}(\mathfrak{C}) / \widetilde{\mathfrak{W}}$. Since $\mathfrak{W}_{1} \cap \mathfrak{S}_{\mathfrak{Z}} \supset$ 3, it follows that $\left|\mathfrak{W}_{1} \cap \mathfrak{W}_{1}^{H}\right| \geqq 9$. Clearly, $\mathfrak{W}_{1} \neq \mathfrak{W}_{1}^{H}$, since $\left\langle\overline{\left\langle\mathfrak{D}, \mathfrak{Q}^{H}\right.}\right\rangle$ is nonabelian. Hence, $\widetilde{\mathfrak{W}}$ is elementary of

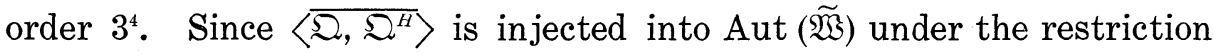
map, it follows readily that $\left\langle\overline{\mathfrak{\Omega}, \mathfrak{\Omega}^{H}}\right\rangle$ is the central product of two quaternion groups, each of which necessarily admits $\mathfrak{B}$. In particular, $\left\langle\overline{\mathfrak{D}, \mathfrak{\Omega}^{H}}\right\rangle^{\prime}$ is of order 2 and inverts $\mathfrak{W}_{1} \cap \mathfrak{S}$. Since no involution of $\mathbb{B}$ centralizes $\mathfrak{W}_{1} \cap \mathfrak{F}$, it follows that $\overline{\mathfrak{Q}_{0}}$ is extra special of order 32 . Hence, $\left[\mathfrak{Q}^{\mathfrak{S}_{0}}, \boldsymbol{O}_{3}(\mathbb{E})\right]$ is elementary of order $3^{4}$. This implies that $\boldsymbol{O}_{3}(\mathfrak{S})$ contains $\left[\mathfrak{D}^{\mathfrak{S}_{0}}, \boldsymbol{O}_{3}(\mathfrak{C})\right] \times \mathfrak{B}_{0}$, an elementary subgroup of order $3^{5}$. Hence, $w \geqq 3$.

Write $\boldsymbol{O}_{3}(\mathfrak{E})=\mathfrak{X} \times \mathfrak{Y}$, where

$$
\mathfrak{X}=\left[\boldsymbol{O}_{3}(\mathfrak{C}), \mathfrak{\Omega}^{\mathfrak{S}_{0}}\right] \text { and } \mathfrak{Y}=\boldsymbol{O}_{3}(\mathfrak{S}) \cap C\left(\mathfrak{\beth}^{\mathfrak{L}_{0}}\right) \text {. }
$$

Thus, $\mathfrak{Y}_{0}$ normalizes both $\mathfrak{X}$ and $\mathfrak{Y}$. Suppose $Y \in \mathfrak{Y} \cap \mathfrak{S}$. Then

$$
\left[Y, \mathfrak{S}_{0}\right] \subseteq 3 \cap \mathfrak{Y}=1, \quad \text { so } \quad Y \in Z\left(\mathfrak{S}_{0}\right)=8 \times \mathfrak{B}_{0}
$$

Hence, $\mathfrak{Y} \cap \mathfrak{S}_{\mathfrak{Z}}=\mathfrak{B}_{0}$. Since $\left[\mathfrak{Y}, \mathfrak{S}_{0}\right] \subseteq \mathfrak{S C}_{\mathcal{C}}$, it follows that $\left[\mathfrak{Y}, \mathfrak{S}_{0}\right] \subseteq \mathfrak{B}_{0}$. Since $\mathfrak{\Omega}^{\mathfrak{S}_{0}}$ is absolutely irreducible on $\mathfrak{X}$, it follows that

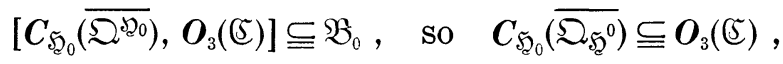

since $\boldsymbol{O}_{3}(\mathfrak{S})=\boldsymbol{O}_{3}\left(\mathfrak{\complement} \bmod \mathfrak{B}_{0}\right)$.

Clearly, $\mid \mathfrak{S}_{0}: C_{\mathfrak{S}_{0}}\left(\overline{\left.\mathfrak{\Omega}^{\mathfrak{F}_{0}}\right)} \mid=3^{a}, a=1\right.$ or 2 , since $\overline{\mathfrak{D}^{\mathfrak{S}_{0}}}$ is extra special of order 32. If $a=1$, then $\mathfrak{S}_{0} \cap \boldsymbol{O}_{3}(\mathfrak{E})$ is of index 9 in $\mathfrak{S}$, so is nonabelian since $w \geqq 3$. This is impossible, since $3 \nsubseteq D\left(\boldsymbol{O}_{3}(\mathfrak{E})\right)$.

Suppose $a=2$. Set $\mathfrak{A}=\mathfrak{H}_{0} \cap \boldsymbol{O}_{3}(\mathfrak{C})$. Since $\mathfrak{A}$ is abelian, $w=3$. Thus, $\mathfrak{A} \in \mathscr{S}_{\text {con }}(\mathfrak{Y})$. Let $\mathfrak{A}_{1}=\mathfrak{X} \cap \mathfrak{A}$, so that $27 \geqq\left|\mathfrak{A}_{1}\right| \geqq 9$. Suppose $\left|\mathfrak{A}_{1}\right|=9$. Let $\mathfrak{A}_{2}$ be a complement to $\mathfrak{A}_{1}$ in $\mathfrak{X}$, so that $\left|\mathfrak{A}_{2}\right|=9$, and $\mathfrak{A}_{2} \cap \mathfrak{S}=1$. Since $\mathfrak{U}_{2}$ centralizes $\mathfrak{A}$, we get $\left[\mathfrak{H}_{2}, \mathfrak{N}_{2}\right] \subseteq \mathfrak{S} \cap \boldsymbol{C}(\mathfrak{T})=\mathfrak{A}$, so that $\left[\mathfrak{H}_{\mathcal{E}}, \mathfrak{A}_{2}, \mathfrak{A}_{2}\right]=1$. Thus, $\left[\mathfrak{Q}_{3}^{\prime}(\mathfrak{N}), \mathfrak{N}_{2}\right]$ is a 2 -group on which $\mathfrak{U}_{2}$ is faithfully represented. This violates Lemma 9.3. Hence, $\mathfrak{A}_{1}$ is of order $3^{3}$, so that $\mathfrak{U}=\mathfrak{U}_{1} \times \mathfrak{B}_{0}$. Suppose $\mathfrak{B}_{0} \subset \mathfrak{Y}$. Let $\mathfrak{Y}_{1}$ be a subgroup of $\mathfrak{Y}$ of order 9 which contains $\mathfrak{B}_{0}$. Then $\mathfrak{X} \mathfrak{Y}_{1}$ is abelian of order $3^{6}$, and $\left[\mathfrak{S}_{\mathfrak{E}}, \mathfrak{X} \mathfrak{Y}_{1}\right] \subseteq \mathfrak{S} \cap \boldsymbol{C}(\mathfrak{U})=\mathfrak{U}$, so that $\left[\mathfrak{K}, \mathfrak{X} \mathfrak{Y}_{1}, \mathfrak{X} \mathfrak{Y}_{1}\right]=1$. It follows that $\left[\boldsymbol{Q}_{3}^{1}(\mathfrak{R}), \mathfrak{X Y} \mathfrak{Y}_{1}\right]$ is a 2-group on which $\mathfrak{X} \mathfrak{Y}_{1} / \mathfrak{A}$ is faithfully represented. This again violates Lemma 9.3 , so $\mathfrak{V}=\mathfrak{B}_{0}$. 
Since $\mathfrak{Y}=\mathfrak{B}_{0}$ and $a=2, \boldsymbol{O}_{3}(\mathfrak{C})$ is elementary of order $3^{5}$ and $\left|\mathfrak{P}_{0}\right|=3^{7}$. Lemma 5.2 implies that if $U$ is any element of $\mathbb{E} / O_{3}(\mathbb{E})$ of order 3 , then $\boldsymbol{C}(U) \cap \boldsymbol{O}_{3}(\sqrt{5})$ is of order at most $3^{3}$.

Suppose by way of contradiction that $\mathfrak{U}$ is an elementary subgroup of $\mathfrak{P}_{0}$ of order $3^{5}$ which is distinct from $\boldsymbol{O}_{3}(\mathfrak{E})$. By the previous paragraph, we conclude that $\mathfrak{u} \cap \boldsymbol{O}_{3}(\boldsymbol{C})$ is of order $3^{3}$, and that if $U \in \mathfrak{U}-\boldsymbol{O}_{3}(\mathfrak{E})$, then $\boldsymbol{O}_{3}(\mathfrak{E}) \cap \boldsymbol{C}(U)=\boldsymbol{O}_{3}(\mathfrak{S}) \cap \mathfrak{U}$. Let $\mathfrak{u}_{0}$ be a complement to $\mathfrak{U} \cap \boldsymbol{O}_{3}(\mathfrak{E})$ in $\mathfrak{U}$. Thus, $\mathfrak{U}_{0}$ is faithfully represented on $\boldsymbol{Q}_{3}^{\mathfrak{l}}(\Re)$, the central product of two quaternion groups. Let $\Re$ be a quaternion subgroup of $\boldsymbol{Q}_{3}^{1}(\Re)$, and let $\mathfrak{U}_{1}=\boldsymbol{C}(\Re) \cap \mathfrak{U}_{0}$. Thus, $\mathfrak{U}_{1}$ is of order 3 . By Lemma 3.7 of [20], $\Re$ is faithfully represented on $\boldsymbol{O}_{3}(\mathfrak{S}) \cap \boldsymbol{C}\left(\mathfrak{U}_{1}\right)$. This is absurd, since $\mathfrak{U}_{0}$ centralizes $\boldsymbol{O}_{3}(\mathfrak{E}) \cap \boldsymbol{C}\left(\mathfrak{U}_{1}\right)$. We conclude that $\boldsymbol{O}_{3}(\mathfrak{C})$ is the only elementary subgroup of its order in $\mathfrak{P}_{0}$.

Since $\left|\mathfrak{P}_{0}\right|=|\mathfrak{K}|=3^{7}$ and since $\mathfrak{P}_{0}$ is obviously not extra special it follows that $\mathfrak{P}_{0}$ is not a $S_{3}$-subgroup of $\left(\mathcal{S}_{0}\right.$. Hence, $\mathfrak{P}_{0}$ is not a $S_{3}$ subgroup of $N\left(O_{3}(\mathbb{E})\right)$. Hence, $\boldsymbol{A}_{\circlearrowleft}\left(O_{3}(\mathfrak{E})\right)$ is a solvable subgroup of $G L(5,3)$ with $S_{3}$-subgroups of order at least 27 and with no nonidentity normal 3-subgroups. There are no such groups. The proof of Lemma 9.6 is complete.

\section{Lemma 9.7. Every involution in $\mathfrak{R}$ centralizes 3.}

Proof. Suppose false. Let $\mathscr{F}_{1}=\Omega_{1}\left(\mathscr{F}_{\mathcal{E}}\right)$, so that $\mathscr{F}_{1}$ is extra special of exponent 3 and width $w \geqq 2$. Let $\mathfrak{S}_{0}=C_{\mathfrak{S}_{1}}(I)$ and let $\mathfrak{S}_{2}$ be the set of elements of $\mathfrak{S}_{1}$ inverted by $I$. Here $I$ is an involution of $\mathfrak{R}$ which does not centralize 3 . Since $3 \subseteq \mathscr{S}_{2}$, it follows that $\mathfrak{S}_{0}$ is abelian. Since $I$ centralizes $\left[H, H^{\prime}\right]$ for all $H, H^{\prime}$ in $\mathscr{S}_{2}$, it follows that $\left\langle\mathscr{S}_{2}\right\rangle$ is abelian. Hence, $\mathfrak{S}_{2}=\left\langle\mathfrak{S}_{2}\right\rangle$. As is well known, $\mathfrak{S}_{1}=\mathfrak{S}_{0} \mathfrak{S}_{2}$ and $\mathfrak{S}_{0} \cap \mathfrak{S}_{2}=$ 1. Hence, $\mathfrak{S}_{2}$ is elementary of order $3^{w+1}$ and $\mathfrak{S}_{0}$ is elementary of order $3^{w}$.

By Lemmas 7.5 and 9.6, there is a subgroup $\mathfrak{M}$ in $\mathscr{A C S}$ (S) with $\mathfrak{S}_{0} \subseteq \mathfrak{M}$ such that $\mathfrak{M}$ satisfies Hypothesis 7.2 and $p=2$. Let $w_{1}$ be the width of $\boldsymbol{O}_{2}(\mathfrak{M})$. Hence, $w \leqq w_{1} \leqq 4$, the first inequality holding since $\mathscr{S}_{0}$ is faithfully represented on $\boldsymbol{O}_{2}(\mathfrak{M})$, the second inequality holding by Lemma 7.5 .

Suppose $w \geqq 3$. Hence, $w_{1} \geqq 3$. If $H \in \mathfrak{S}_{0}^{\ddagger}$ and $C(H) \cap \boldsymbol{O}_{2}(\mathfrak{M})$ contains a four-subgroup $\mathfrak{B}$ containing $l_{1}\left(\boldsymbol{Z}\left(\boldsymbol{O}_{2}(\mathfrak{M})\right)\right)$, then by Lemma 7.2 , both $\langle H, 3\rangle$ and $\mathfrak{B}$ satisfy the hypothesis of Lemma 7.4 , so $C(H)$ is nonsolvable. This is impossible, so $H$ is not available. This implies that $w=2$.

Suppose $\mathfrak{S}=\mathfrak{P}$. In this case, if $H$ is any element of order 3 in $\mathfrak{S}$, then 3 char $C_{\mathfrak{S}}(H)$. This implies immediately that 3 is weakly closed in $\mathfrak{P}$, which in turn implies that $\mathfrak{N}$ contains the centralizer of each of its nonidentity 3-elements. This implies that $\boldsymbol{O}_{2}(\mathfrak{M}) \subseteq \mathfrak{R}$, which 
is not the case. Hence $\mathfrak{S C} \subset \mathfrak{P}$.

Since every 3,5-subgroup of $S_{4}(3)$ is either a 3-group or a 5-group, it follows from the preceding paragraph that $\mathfrak{N}$ is a 2,3-group, $S_{4}(3)$ being a 2,3,5-group. By Lemma 9.3 , it then follows that $O^{3^{\prime}}(\mathfrak{R}) / \mathfrak{S} \cong$ $S L(2,3)$. Furthermore, if $J$ is an involution of $O^{3^{\prime}}(\mathfrak{R})$, then $C_{\mathfrak{5}}(J) \triangleleft \mathfrak{R}$. It follows that $J$ inverts $\mathfrak{S} / Z(\mathscr{S})$.

If $I$ centralizes $O^{3^{\prime}}(\mathfrak{R}) / \boldsymbol{O}^{3^{\prime}}(\mathfrak{R})^{\prime}$, we conclude that $I$ centralizes $\boldsymbol{O}^{3^{\prime}}(\mathfrak{R}) / \mathfrak{S}$. But $C(I) \subseteq \mathfrak{M}$, so in particular, $C_{\mathfrak{R}}(I) \subseteq \mathfrak{M}$. Since $I$ centralizes $\boldsymbol{O}^{3^{\prime}}(\mathfrak{N}) / \mathfrak{S}$, it follows that $I$ centralizes a $S_{2}$-subgroup $\mathfrak{Q}$ of $\boldsymbol{O}^{3^{\prime}}(\mathfrak{R})$. Hence, $\mathfrak{Q}$ normalizes $\mathfrak{S}_{0}$. Hence, $\mathfrak{Q} \mathfrak{S}_{0}$ is of index 3 in $C(I) \cap \boldsymbol{O}^{\prime \prime}(\mathfrak{R})$. Let $\mathfrak{\Omega}^{\prime}=$ $\langle J\rangle$. By the preceding paragraph, $\mathscr{Z}$ is faithfully represented on $\mathfrak{S}_{0}$. Thus, $\mathfrak{D}=C(I) \cap N(\mathfrak{D}) \cap \boldsymbol{O}^{3}(\mathfrak{R}) \cong S L(2,3)$ and $\mathfrak{D}$ is faithfully represented on $\mathfrak{S}_{0}$.

Since $\mathfrak{S}_{0}$ is faithfully represented on $\boldsymbol{O}_{2}(\mathfrak{M})$, so is $\mathfrak{S}_{0} \mathfrak{D}$. Since $\mathfrak{S}_{0} \cap \mathfrak{D}=1, S_{3}$-subgroups of $\mathfrak{S}_{0} \mathfrak{D}$ are of exponent 3 . Since the four subgroups of $\mathfrak{S}_{0}$ of order 3 are permuted transitively by $\mathfrak{Q}$, it follows that $w_{1} \geqq 4$. Hence, $w_{1}=4$ and $\boldsymbol{O}_{2}(\mathfrak{M})$ is extra special. Let $\mathfrak{P}_{0}$ be a $S_{3}$-subgroup of $\mathfrak{S}_{0} \mathfrak{D}$. We can choose $P$ in $\mathfrak{P}_{0}-\mathfrak{S}_{0}$ such that $C(P) \cap \boldsymbol{O}_{2}(\mathfrak{M})$ contains a four-group. Since $C_{\mathfrak{R}}(P)$ clearly contains an element of $\mathscr{C}(3)$, Lemma 7.4 is violated. We conclude that $I$ does not centralize $O^{3^{\prime}}(\mathfrak{R}) /$ $\boldsymbol{O}^{3^{\prime}}(\mathfrak{N})^{\prime}$.

Since $\operatorname{Aut}(\boldsymbol{Z}(\mathfrak{E}))$ is abelian, the preceding paragraph implies that $\boldsymbol{Z}(\mathfrak{E})=\boldsymbol{Z}(\mathfrak{P})$.

Since $\boldsymbol{O}_{2}(\mathfrak{M}) \nsubseteq \Re$, we can choose $H$ in $\mathfrak{S}_{0}^{*}$ such that $\boldsymbol{C}(H) \nsubseteq \mathfrak{R}$.

Let $|\boldsymbol{Z}(\mathfrak{S})|=3^{a}$, and suppose $a \geqq 2$. Let $\tilde{\mathfrak{B}}$ be a $S_{3}$-subgroup of $\boldsymbol{C}_{\mathfrak{R}}(H)$. Thus, $\boldsymbol{Z}(\mathfrak{S}) \subseteq \boldsymbol{Z}(\tilde{\mathfrak{P}})$, and $\boldsymbol{B}=\tilde{\sigma}^{a-1}(\boldsymbol{Z}(\tilde{\mathfrak{P}}))$ char $\tilde{\mathfrak{P}}$, whence $\tilde{\mathfrak{P}}$ is a $S_{3}$-subgroup of $C(H)$. By Lemma 7.2 applied to $\langle H, 3\rangle$, it follows that 3 centralizes $\boldsymbol{O}_{3^{\prime}}(\boldsymbol{C}(H))$, and so

$$
\left[\boldsymbol{O}_{3^{\prime}}(\boldsymbol{C}(H)), \boldsymbol{C}_{\mathfrak{S}}(H)\right] \subseteq \mathfrak{S} \cap \boldsymbol{O}_{3^{\prime}}(\boldsymbol{C}(H))=1 .
$$

By Lemma 9.4, we have $\boldsymbol{O}_{3^{\prime}}(\boldsymbol{C}(H))=1$. Let $\tilde{\mathfrak{P}}_{1}=\boldsymbol{O}_{3}(\boldsymbol{C}(H)) \subseteq \tilde{\mathfrak{P}}$. Thus, $\boldsymbol{Z}(\mathfrak{S}) \subseteq \boldsymbol{Z}\left(\tilde{\mathfrak{P}}_{1}\right)$, and we get $\mathbb{Z}=\sigma^{a-1}\left(\boldsymbol{Z}\left(\tilde{\mathfrak{P}}_{1}\right)\right)$, whence $\boldsymbol{C}(H) \subseteq \mathfrak{R}$. This contradiction forces $a=1, Z(\mathfrak{S})=3,|\mathfrak{P}|=3^{6}$.

Throughout the remainder of this lemma, the following notation is used: $\mathfrak{Q}$ is a $S_{2}$-subgroup of $O^{3^{\prime}}(\mathfrak{R})$ normalized by $I$. Since $\mathfrak{Q}$ is a quaternion group, our preceding information implies the existence of an element $Q$ in $\mathfrak{Q}$ of order 4 such that $I Q I=Q^{-1}$. Let $J=Q^{2}$. Thus, $J$ centralizes 3 and inverts $\mathfrak{S} / 3$.

We argue that (S) is not 3-normal. Namely, for some $H$ in $\mathfrak{S}_{0}^{\#}$, we have $\mathfrak{E}=\boldsymbol{C}(H) \nsubseteq \mathfrak{R}$. If $|\mathfrak{E}|_{3}=|\mathfrak{S}|_{3}$, then $\langle H\rangle$ is a conjugate of 3 contained in $\mathfrak{S}$, and we are done. Otherwise, it is clear that $\mathfrak{E} \cap \mathfrak{N}$ contains a $S_{3}$-subgroup of $\sqrt{ } \sqrt{ }$ and since $3 \subseteq \mathfrak{C}, \boldsymbol{O}_{3}(\mathfrak{E})$ contains at least two conjugates of 3 . As $\boldsymbol{O}_{3}(\mathfrak{C}) \subseteq \Re$, we again are done.

We next argue that 3 is not weakly closed in $\mathfrak{F}_{\text {. }}$. Choose $G$ in 
(8) such that $3_{1}=3^{G} \subseteq \mathfrak{P}$ and $3 \neq 3_{1}$. If $Z_{1} \subseteq \mathfrak{K}$, we are done. Otherwise, let $\mathfrak{P}_{0}=\boldsymbol{C}_{\mathfrak{P}}\left(\Re_{1}\right)=3_{1} \times \boldsymbol{C}_{\mathfrak{\mathfrak { H }}}\left(\mathfrak{Z}_{1}\right)$. Since $\mathfrak{P}_{0}$ is not a $S_{3}$-subgroup of (3) but $\mathfrak{P}_{0}$ is a $S_{3}$-subgroup of $C_{\mathfrak{N}}\left(B_{1}\right)$, it follows that $3 \mathrm{ch} /$ ar $\mathfrak{P}_{0}$. This implies that $\mathfrak{P}_{0}$ is elementary. Clearly, $27 \leqq\left|\mathfrak{P}_{0}\right| \leqq 81$, since $w=2$. We assume without loss of generality that $\mathfrak{P} \cap \mathfrak{P}^{G}=\mathfrak{P}_{0}$. If $3 \subseteq \mathfrak{S g}^{G}$, we are done, so we may assume that $3 \nsubseteq \mathfrak{S C}^{G}$, which yields $\mathfrak{P}_{0}=$ $3 \times\left(\mathfrak{P}_{0} \cap \mathfrak{S}^{G}\right)$. In particular, $\mathfrak{S} \cap \mathfrak{S}^{G} \neq 1$; let $\mathfrak{B}=\mathfrak{S} \cap \mathfrak{S}^{G}$, a group of order at least 3. Suppose $|\mathfrak{B}|=3$. Let $\Re=C(B)$, so that $|\Re \cap \Re|=$ $\left|\Re \cap \mathfrak{P}^{6}\right|=3^{5}$. If $|\Re|_{3}=3^{6}$, we are done, so we may assume that $|\Re|_{3}=3^{5}$. In this case, we see that $\left|\boldsymbol{O}_{3}(\Re)\right|=3^{4}$, which implies that $\left|O_{3}(\Omega) \cap \mathscr{S}_{\mathcal{C}}\right| \geqq 27,\left|O_{3}(\Omega) \cap \mathscr{S}_{\mathcal{C}}{ }^{G}\right| \geqq 27$. Hence, $\left|\mathfrak{S} \cap \mathscr{S}^{G}\right| \geqq 9$, contrary to assumption. Thus, we may assume that $|\mathfrak{B}|=9$. We may also assume that $\mathfrak{B}$ contains no conjugate of $\mathfrak{3}$. We have $\mathfrak{P}_{0}=3 \times \mathfrak{Z}_{1} \times \mathfrak{B}$. We argue that $\mathfrak{P}_{0} \triangleleft\left\langle\mathfrak{P}, \mathfrak{P}^{a}\right\rangle . \quad$ Namely, let $\mathfrak{P}_{0} \subset \mathfrak{P}_{1} \subset \mathfrak{P}$. If $\mathfrak{P}_{0}$ char $\mathfrak{P}_{1}$, then clearly $\mathfrak{P}$ normalizes $\mathfrak{P}_{0}$. Suppose $\mathfrak{P}_{0} \operatorname{ch} /$ ar $\mathfrak{P}_{1}$. Then $\mathfrak{P}_{1}=\mathfrak{P}_{0} \mathfrak{P}_{0}^{*}$, where $\mathfrak{P}_{0}^{*}$ is elementary of order $3^{4}$. Hence, $Z\left(\mathfrak{P}_{1}\right)=\mathfrak{P}_{0} \cap \mathfrak{P}_{0}^{*}$ is of order 27. This implies that $\mathfrak{P}_{1}^{\prime}$ is of order 3 . Since $\mathfrak{P}_{1} \cap \mathfrak{F}_{2}$ is nonabelian, we have $\mathfrak{P}_{1}^{\prime}=3$. Thus, $\mathfrak{Z}_{1}$ centralizes $\left(\mathfrak{P}_{1} \cap \mathfrak{S}\right) / 3$. This is not the case, since involutions of $O^{3^{\prime}}(\mathfrak{R})$ invert $\mathscr{S} / 3$, so that the action of $Z_{1}$ on $\mathfrak{S}_{2} / 3$ is given either by $J_{3} \oplus J_{1}$ or by $J_{2} \oplus J_{2}$. By symmetry, we have $\mathfrak{P}_{0} \triangleleft\left\langle\mathfrak{P}, \mathfrak{P}^{G}\right\rangle$. It is easy to verify that $\boldsymbol{O}_{3}\left(N\left(\mathfrak{P}_{0}\right)\right)$ is of order $3^{5}$, which implies that $\left|\mathfrak{S} \cap \mathfrak{S E}^{G}\right| \geqq 27$, the desired contradiction.

Since all parts of Hypothesis 9.3 are satisfied, Lemma 9.5 is violated. The proof of the lemma is complete.

LEMMA 9.8. $\mathfrak{N}$ is the only element of $\mathscr{L S}(\mathbb{S})$ which contains $\mathfrak{S}$.

Proof. Suppose false. Choose $\Re \in \mathscr{S}_{\mathcal{U}} /(\mathfrak{S})$ so that $\mathfrak{S} \cong \Re \nsubseteq \mathfrak{R}$, and with this restriction, minimize $|\Omega|$. Since $И(\mathfrak{S})$ contains only 1 , Lemma 0.7.6 implies that $l_{3}(\Re) \leqq 2$. If $l_{3}(\Re)=1$, then $3 \triangleleft \Re$, contrary to assumption. Hence, $l_{3}(\Re)=2$; and $\Re$ is a $3, p$-group for some prime p. Furthermore, $\mathfrak{S}$ acts irreducibly on $\boldsymbol{O}_{3, p}(\Re) / \boldsymbol{D}\left(\boldsymbol{O}_{3, p}\left(\Re \bmod \boldsymbol{O}_{3}(\Re)\right)\right)$. Let $\mathfrak{S}_{0}=\mathfrak{S} \cap \boldsymbol{O}_{3}(\Re), \mathfrak{B}=\Omega_{1}\left(\boldsymbol{Z}\left(\boldsymbol{O}_{3}(\Re)\right)\right)$. By Lemma 9.4, $\mathfrak{B} \leqq \mathfrak{S C}_{\text {, so }}|\mathfrak{B}| \leqq$ 9. Thus, $O_{3, p}(\Re) / O_{3}(\Re)$ is a quaternion group whose involution inverts $\mathfrak{B}$. Since $3 \subset \mathfrak{B}$, Lemma 9.7 is violated. The proof is complete.

Lemma 9.9. Every involution $I$ of $\mathfrak{N}$ inverts $\mathfrak{S} / \boldsymbol{Z}(\mathfrak{S})$.

Proof. By Lemma 9.7, I centralizes 3, so centralizes $\boldsymbol{Z}(\mathfrak{S})$. If the lemma is false, then $C_{\mathfrak{H}}(I)$ contains a subgroup $\mathfrak{U}$ of type $(3,3)$ with $\mathfrak{2} \supset 3$. This violates Lemma 9.3 (iii).

Lemma 9.10. If $\mathfrak{U} \in \mathscr{A}_{4}(\mathfrak{P})$, then $\mathfrak{N}$ is the only element of $\mathscr{H} S(\mathbb{S})$ which contains $\mathfrak{A}$. 
Proof. As in $O$, let $\mathscr{A}_{1}=\{\mathfrak{A} \mid \mathfrak{A}$ is a 3 -subgroup of $\mathfrak{N}$, $\mathfrak{A}$ contains an element of $\mathscr{S C C}_{\mathfrak{S}}\left(\mathfrak{P}^{N}\right)$ for some $N$ in $\mathfrak{R}, \mathscr{A}_{n+1}=\{\mathfrak{X} \mid \mathfrak{A}$ is a 3subgroup of $\mathfrak{N}$, $\mathfrak{X}$ contains a subgroup $\mathfrak{B}$ of type $(3,3), C_{\mathfrak{R}}(B)$ contains an element of $\mathscr{A}_{n}$ for all $B$ in $\left.\mathfrak{B}\right\}$. Among all $\mathfrak{A} \in \mathscr{A}_{4}$ which violate the conclusion of the lemma, maximize $|\mathfrak{A} \cap \mathfrak{S}|$, and with this restriction, maximize $|\mathfrak{U}|$. By Lemma 9.8, $\mathfrak{S} \nsubseteq \mathfrak{A}$. Let $\mathfrak{M} \in \mathscr{M C S}(\mathbb{S})$ with $\mathfrak{X} \subseteq \mathfrak{M}, \mathfrak{M} \neq \mathfrak{R}$. By maximality of $|\mathfrak{U}|$, it follows that $\mathfrak{X}$ is a $S_{3}$-subgroup of $\mathfrak{M}$. We can therefore choose a prime $q$ and a $q$-subgroup $\mathfrak{Q}$ of $\mathfrak{M}$ permutable with $\mathfrak{A}$ such that $\mathfrak{L}=\mathfrak{U} \mathfrak{Q}$ is not contained in $\mathfrak{N}$. Let $\Omega$ be minimal with these properties. By Lemma 0.7.6, $l_{3}(\Omega) \leqq 2$.

We first show that $\boldsymbol{O}_{q}(\mathfrak{Q}) \cong \mathfrak{R}$. Suppose $\mathfrak{U} \cap \mathfrak{S}$ is noncyclic. Let $\mathfrak{B}$ be a subgroup of $\mathfrak{A} \cap \mathfrak{F}$ of type $(3,3)$. It suffices to show that $C(B) \subseteq \mathfrak{R}$ for all $B \in \mathfrak{B}^{\sharp}$. Suppose false. Then maximality of $|\mathfrak{A} \cap \mathfrak{S}|$ yields $|\mathfrak{S}: \mathfrak{U} \cap \mathfrak{S}| \leqq 3$. In this case, $И(\mathfrak{U} \cap \mathfrak{S})=1$, so $\boldsymbol{O}_{q}(\mathfrak{L})=1$. Thus, we may assume that $\mathfrak{U} \cap \mathfrak{F}$ is cyclic. Since $w \geqq 2$, it follows that if $P$ is any element of $\mathfrak{P}$ of order 3 , then $\boldsymbol{C}_{\mathfrak{5}}(P)$ is noncyclic. Hence, every subgroup of $\mathfrak{R}$ of type $(3,3)$ is in $\mathscr{A}_{4}$. Since $\mathfrak{A}$ contains a subgroup of type $(3,3)$, maximality of $|\mathfrak{U} \cap \mathfrak{S}|$ implies that $C(A) \subseteq \mathfrak{N}$ for all elements $A$ of $\mathfrak{X}$ of order 3 . Thus, in all cases, we have $\boldsymbol{O}_{q}(\mathfrak{R}) \cong \mathfrak{R}$.

By minimality of $\mathfrak{Q}, \boldsymbol{O}_{q, 3}(\mathfrak{Q})=\boldsymbol{O}_{q}(\mathfrak{Q}) \times \boldsymbol{O}_{3}(\mathfrak{Q})$. Since $l_{3}(\mathfrak{R}) \leqq 2$, it follows that $l_{3}(\mathbb{R})=2$, by maximality of $|\mathfrak{A}|$ and the structure of $\boldsymbol{O}_{q, 3}(\mathfrak{Q})$. Since $\boldsymbol{D}(\mathfrak{Q})$ is permutable with $\mathfrak{A}$, we get $\boldsymbol{D}(\mathfrak{\Omega}) \subseteq \mathfrak{R}$, by minimality of $\mathfrak{\Omega}$.

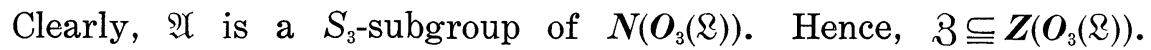
Since $\mathfrak{O} \boldsymbol{O}_{3}(\mathbb{R}) \triangleleft \mathscr{L}$, and since $\mathfrak{U}$ is a $S_{3}$-subgroup of $\boldsymbol{N}\left(\boldsymbol{O}_{3}(\mathbb{R})\right)$, and since $\mathfrak{S} \nsubseteq \mathfrak{A}$, it follows that $\mathfrak{S} \cap \mathfrak{U}$ acts nontrivially on $\boldsymbol{Q}_{3}^{1}(\mathbb{R})$, but trivially on every proper $\mathfrak{A}$-invariant subgroup of $\boldsymbol{Q}_{3}^{1}(\mathfrak{Q})$. Since $\boldsymbol{D}(\mathfrak{Q})$ centralizes 3 , it follows that $\boldsymbol{D}(\mathfrak{Q})$ centralizes $\mathfrak{B}=Z^{\mathfrak{R}}$.

If $q \geqq 5$, then maximality of $\mathfrak{A}$ and Theorem 1 of [39] imply that $\mathfrak{U}=\mathfrak{P}$, against Lemma 9.8. Hence, $q=2$. We may apply Theorem 1 of [39] once again and conclude that $\boldsymbol{D}(\Omega) \neq 1$. By Lemma 9.9, each element of $\boldsymbol{D}(\mathfrak{\Omega})^{\sharp}$ inverts $\mathfrak{K} / 3(\mathfrak{S})$. Since $\boldsymbol{Z}(\mathfrak{K})$ is a normal cyclic subgroup of $\mathfrak{P}$, it follows that $\mathfrak{U} \cap \boldsymbol{Z}(\mathfrak{S}) \subseteq \boldsymbol{O}_{3}(\mathfrak{L})$. Since $\mathfrak{A} \cap \mathfrak{\mathcal { F }} \varsubsetneqq \boldsymbol{O}_{3}(\mathfrak{L})$, choose $H \in \mathfrak{U} \cap \mathfrak{S}-\boldsymbol{O}_{3}(\mathfrak{Q})$. Let $I$ be the element in $\boldsymbol{D}(\mathfrak{\Omega})^{*}$. Then $H^{I}=H^{-1} H_{0}$ with $H_{0}$ in $Z(\mathfrak{S})$. Since $H_{0} \in \mathfrak{A}$, it follows that $[H, I]$ is contained in $\mathfrak{U} \cap \mathfrak{S}_{\mathcal{C}} \cap O_{3, q}(\mathfrak{R}) \subseteq \mathfrak{A} \cap \boldsymbol{O}_{3}(\mathbb{R})$. This violates the fact that $\mathfrak{U} \cap \mathfrak{S}_{\mathfrak{Z}} \equiv \boldsymbol{O}_{3}(\mathbb{R})$. The proof is complete.

It is now easy to show that Hypothesis 9.2 is not satisfied. Otherwise, $\mathfrak{R}$ contains a four-subgroup $\mathfrak{T}$. But by Lemma 9.9, each element of $\mathfrak{T}^{\sharp}$ inverts $\mathfrak{S} / Z(\mathfrak{Z})$. This is not possible, since $\mathfrak{S}=$ $\left\langle C_{\mathfrak{S}}(J) \mid J \in \mathfrak{T}^{\sharp}\right\rangle$.

The remaining lemmas in this section are proved on the hypothesis 
that $\mathfrak{S}$ contains a noncyclic characteristic abelian subgroup.

Among all noncyclic normal elementary subgroups of $\mathfrak{N}$, let $\leftleftarrows$ be minimal. Thus, $\mathbb{E} / 3$ is a chief factor of $\Re$. Let $\mathscr{C}: \mathbb{F} \supset 3 \supset 1$. We will show that $\boldsymbol{A}_{\mathscr{G}}(\mathscr{C})=\boldsymbol{A}(\mathscr{C})$. First, suppose $\mathbb{F}$ is not 3-reducible in $\Re$. Let $\mathfrak{L}=\boldsymbol{O}_{3}(\mathfrak{R} \bmod C(\mathbb{F}))$. Since $\mathbb{E} / 3$ is a chief factor of $N$, we have $[\mathbb{R}, \mathbb{F}]=3$, and $3=C_{\mathfrak{F}}(\mathbb{R})$. These equalities imply immediately that $\mathbb{Q}$ maps onto $\boldsymbol{A}(\mathscr{C})$. Suppose $\mathbb{F}$ is 3 -reducible in $\mathfrak{R}$. Let $\mathbb{L}=$ $\boldsymbol{O}_{3^{\prime}}(\mathfrak{N} \bmod \boldsymbol{C}(\mathfrak{F}))$. Then $3=\boldsymbol{C}_{\mathfrak{F}}(\mathfrak{R})$ and [R, [] admits $\boldsymbol{N}_{\mathfrak{R}}(\mathfrak{R})=\mathfrak{R}$. Since $\boldsymbol{A}_{\mathfrak{Q}}(\mathfrak{S})$ is a $3^{\prime}$-group it follows that [R, [্] is a normal subgroup of $\mathfrak{R}$ disjoint from 3 . Hence, $[\mathcal{Q}, \mathbb{F}]=1$, since 3 is the only minimal normal subgroup of $\mathfrak{P}$. Since $\mathfrak{F}$ is 3 -reducible in $\mathfrak{N}$ and $\boldsymbol{O}_{3^{\prime}}(\mathfrak{N} \bmod \boldsymbol{C}(\mathfrak{F}))$, it follows that $\mathfrak{F} \subseteq \boldsymbol{Z}(\mathfrak{N})$. This is absurd since 3 is the only minimal normal subgroup of $\mathfrak{P}$. Thus, $\boldsymbol{A}_{\mathscr{S}}(\mathscr{C})=\boldsymbol{A}(\mathscr{C})$.

Throughout the remainder of this section, the following notation is used: $\mathfrak{P}, \mathbb{Z}, \mathfrak{R}$ are as before, and $\mathfrak{F}$ is a noncyclic normal elementary

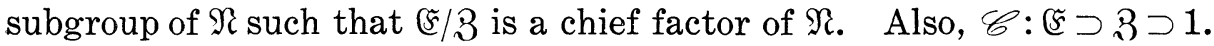

Lemma 9.11. ( i ) If $\mathfrak{S}$ is a 2, 3-subgroup of (S) and $\mathfrak{S}$ contains an element $\mathfrak{A}$ of $\mathscr{E}(3)$, then $\boldsymbol{O}_{2}(\mathfrak{S})=1$.

(ii) If $\mathfrak{U} \in \mathscr{E}(3)$, then $|\boldsymbol{C}(\mathfrak{U})|$ is odd.

Proof. ( i ) Suppose $I$ is an involution in $\boldsymbol{O}_{2}(\Im)$. Since $\mathfrak{A}$ centralizes $\boldsymbol{O}_{2}(\Im)$, Lemmas 7.4 and 5.38 imply that $\boldsymbol{C}(I)$ is nonsolvable.

(ii) Suppose $I$ is an involution of $C(\mathfrak{H})$. Then $\mathfrak{A} \times\langle I\rangle$ violates (i).

Lemma 9.12. ( i ) If I is an involution of $C(3)$, then I inverts $5 / 3$.

(ii) C(3) contains no four-group.

(iii) If $\mathfrak{I}$ is an abelian 2-subgroup of $\mathfrak{R}$, then $\boldsymbol{A}_{\mathfrak{N}}(\mathfrak{I})$ is a 2-group.

Proof. (i) is a consequence of Lemmas 9.11 and 7.3, and (ii), (iii) are consequences of (i).

LEMma 9.13. $\mathfrak{R}$ does not contain a noncyclic abelian subgroup of order 8.

Proof. Suppose false. Let $\mathfrak{\Omega}_{3}^{*}$ be a $S_{2}$-subgroup of $\mathfrak{R}$ permutable with $\mathfrak{P}$, and let $\mathfrak{N}_{0}=\mathfrak{P}_{\mathfrak{\Omega}_{0}^{*}}^{*}$. Let $\mathfrak{\Omega}_{0}=\mathfrak{\Omega}_{0}^{*} \cap{O^{3}}^{\prime}\left(\mathfrak{N}_{0}\right)$. Thus, $\mathfrak{\Omega}_{0}$ is either a quaternion group or $\mathfrak{l}_{0}=1$. Let $\mathfrak{\Omega}$ be a subgroup of $\mathfrak{\Omega}_{3}^{*}$ which contains $\bigcap_{0}$, is permutable with $\mathfrak{P}$, contains a noncyclic abelian subgroup of order 8 , and is minimal with these properties. Let $\mathfrak{R}_{1}=\mathfrak{P} \mathfrak{P}$. Thus, $\mathfrak{Q}$ is abelian of type $(2,4)$ if and only if every 2,3 -subgroup of $\mathfrak{N}$ is 3-closed. If $\mathfrak{\Omega}_{0} \neq 1$, then $|\mathfrak{Q}|=2^{4}$ and $\mathfrak{Q}$ is either the direct product of a group of order 2 and $\mathfrak{\Omega}_{0}$ or $\mathfrak{Q}$ is the central product of a cyclic group of order 4 and $\mathfrak{\Omega}_{0}$. Let $\mathfrak{F} / 3$ be a chief factor of $\mathfrak{R}_{1}$ 


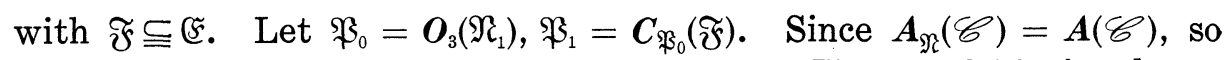
also $\boldsymbol{A}_{\mathfrak{R}_{1}}\left(\mathscr{C}_{0}\right)=\boldsymbol{A}\left(\mathscr{C}_{0}\right)$ where $\mathscr{C}_{0}: \mathfrak{F} \supset \mathbb{Z} \supset 1$. Hence, $\mathfrak{P}_{0} / \mathfrak{F}_{1}$ is also a chief factor of $\mathfrak{N}_{1}$ with the same order as $\mathfrak{F} / \mathfrak{Z}$. If $\mathfrak{Q}^{\prime}=1$, then

$$
\mathfrak{P} \triangleleft \mathfrak{N}_{1}, \mathfrak{Q} \text { is of type }(4,2) \text {, and }|\mathfrak{F}: 3|=9 \text {. }
$$

Suppose $\mathfrak{Q}^{\prime} \neq 1$. If $\mathfrak{\Omega}=\mathfrak{\Omega}_{0} \times \mathfrak{\Omega}_{1}$, where $\left|\mathfrak{Q}_{1}\right|=2$, then

$$
\mathfrak{R}_{1} / \mathfrak{P}_{0} \cong S L(2,3) \times Z_{2} \text {, and }|\mathfrak{F}: 3|=9 \text {. }
$$

Suppose $\mathfrak{Q}$ is the central product of $\mathfrak{\Omega}_{0}$ and a cyclic group of order 4 . Then

$$
\mathfrak{R}_{1} / \mathfrak{P}_{0} \text { is the central product of }
$$$$
S L(2,3) \text { and } Z_{4} \text {, and }|\mathfrak{F}: 3|=3^{4} \text {. }
$$

By Lemmas 5.41 and 9.12, (9.19), (9.20), (9.21) exhaust all possibilities. It is clear from Lemma 9.12 that

$$
\text { if (9.19) holds, a } S_{2,3} \text {-subgroup of } \mathfrak{N} \text { is } 3 \text {-closed . }
$$

We next will show that

$$
\text { every subgroup of } \mathfrak{F} \text { of order } 9 \text { is in } \mathscr{D} \text {. }
$$

To see this, let $\widetilde{F}_{0}$ be a subgroup of $\widetilde{F}$ of order 9 . If $3 \subset \mathfrak{F}_{0}$, then $\mathfrak{F}_{0} \in \mathscr{E}(3) \subseteq \mathscr{D}$. Thus, we may assume that $\mathfrak{F}_{0} \cap 3=1$. Let $\mathfrak{I}$ be an abelian subgroup in $\boldsymbol{U}\left(\mathfrak{F}_{0} ; 2\right)$ and assume by way of contradiction that $\left[\mathfrak{T}, \mathfrak{F}_{0}\right] \neq 1$. We may assume that $\mathfrak{I}$ is a four-group. Let $\mathfrak{F}_{1}=$ $\mathfrak{F}_{0} \cap \boldsymbol{C}(\mathfrak{T})$, a group of order 3 . Let $\mathfrak{F}=\boldsymbol{C}\left(\mathfrak{F}_{1}\right) \supseteqq\langle\mathfrak{F}, \mathfrak{T}\rangle$. Since $m(\mathfrak{F}) \geqq 3$ and $\widetilde{F} \triangleleft \mathfrak{P}$, there is $\mathfrak{A} \in \mathscr{S} \mathscr{C} \mathscr{N}_{3}(\mathfrak{P})$ with $\mathfrak{F} \subseteq \mathfrak{X}$. Hence, $\mathfrak{A} \subseteq \mathfrak{S}=\boldsymbol{C}\left(\mathfrak{F}_{1}\right)$ implies $O_{3^{\prime}}(\mathfrak{C})=1$ by hypothesis (ii) of Theorem 9.1 .

By Lemma $5.5,3 \subseteq \boldsymbol{O}_{3}(\mathfrak{C})$. Let $\mathfrak{B}=\Omega_{1}\left(\boldsymbol{Z}\left(\boldsymbol{O}_{3}(\mathfrak{E})\right)\right)$, and let $\mathfrak{P}^{*}$ be a $S_{3}$-subgroup of $\mathfrak{C}$. Let $\mathfrak{P}^{G}$ be a $S_{3}$-subgroup of $\mathbb{B S}$ which contains $\mathfrak{P}^{*}$. Then $\mathfrak{Z}^{G} \subset \mathfrak{P}^{*}$, so $\mathfrak{Z}^{G} \subseteq \mathfrak{W}$. By Lemma 9.12 (iii), $\mathfrak{I}$ is faithfully represented on $\mathfrak{M}$. Hence, if $F \in \mathfrak{F}_{0}-\mathfrak{F} \cap \boldsymbol{C}(\mathfrak{I})$, then the minimal polynomial of $F$ on $\mathfrak{W}$ is $(x-1)^{3}$. On the other hand, 3 centralizes $\mathfrak{W}$. Since $\mathbb{F} \triangleleft \mathfrak{N}$, the minimal polynomial of $F$ on $\mathfrak{W}$ is a divisor of $(x-1)^{2}$. This contradiction establishes (9.23).

Since $\mathfrak{Q}$ contains an abelian subgroup of type $(2,4)$, we can choose an involution $I$ of $\Omega$ such that $\widetilde{\mho}_{0}=C_{\mathfrak{\Im}}(I)$ is noncyclic. By Lemmas 7.4 and 5.38, $C(I)$ contains no element of $\mathscr{E}(3)$. Hence, $I$ inverts 3 . Thus, in cases $(9.19),(9.20)$ respectively, we have

$$
\mathfrak{F}=\mathfrak{F}_{0} \times 3
$$

In case (9.21), we have

$$
\left|\mathfrak{F}_{0}\right|=\left|C_{\mathfrak{P}_{0} / \mathfrak{F}_{1}}(I)\right|=9 .
$$


Thus, in case (9.21), we have $\left|\boldsymbol{C}_{\mathfrak{P}_{0}}(I)\right| \geqq 3^{4}$.

Let $\&$ be a $S_{2,3}$-subgroup of $C(I)$ which contains $\mathfrak{F}_{0}$. Since $\mathfrak{F}_{0} \in \mathscr{D}$ and since $C(I)$ contains an element of $\mathscr{U}(2)$, there is an element $\mathfrak{M}$ of $\mathfrak{M S}(G)$ which satisfies all the conclusions of Lemma 7.5, contains $\mathfrak{F}_{0}$ and contains a $S_{2}$-subgroup of $\mathcal{R}$. By Lemma $7.5(\mathrm{f}), I \in \boldsymbol{O}_{2}(\mathfrak{M})$.

We will show that

$$
C_{\mathfrak{R}_{1}}(I) \subseteq \mathfrak{M}
$$

By Lemma $7.5(f)$, it suffices to show that $\mathfrak{M}$ contains an $S_{2}$ subgroup of $C(I)$. By construction, $\mathfrak{M}$ contains an $S_{2}$-subgroup of $\mathbb{R}$, which is an $S_{2,3}$-subgroup of $C(I)$. This proves (9.24).

Suppose (9.19) holds. In this case, we have (9.22). Also, (9.19) implies that every element of $\$ S$ of order 3 centralizes an element of $\mathscr{E}(3)$. Let $\mathfrak{F}_{1}$ be a subgroup of $\mathfrak{F}_{0}$ of order 3 such that

$$
\left[O_{2}(\mathfrak{M}) \cap C\left(\mathfrak{F}_{1}\right), \mathfrak{F}_{0}\right]=\mathfrak{\Omega}^{*} \neq 1 .
$$

Thus, $\Omega^{*}$ is a quaternion group and a $S_{2,3}$-subgroup of $C\left(\widetilde{\mho}_{1}\right)$ is not 3closed. By (9.22), $\mathfrak{F}_{1} \nsim 3$. Since $\left|C_{\mathfrak{R}_{1}}\left(\mathfrak{F}_{1}\right)\right|_{3}=|\mathfrak{P}| / 3$, it follows that $\boldsymbol{C}_{\mathfrak{R}_{1}}\left(\widetilde{\mho}_{1}\right)$ contains a $S_{3}$-subgroup of $\boldsymbol{C}\left(\widetilde{\mho}_{1}\right)$. Let $\mathfrak{P}^{*}$ be a $S_{3}$-subgroup of $\boldsymbol{C}_{\mathfrak{R}_{1}}\left(\mathfrak{\mho}_{1}\right)$. Since $\boldsymbol{C}\left(\widetilde{\mho}_{1}\right)$ contains an element of $\mathscr{S}_{\text {on }_{3}}(\mathfrak{P})$, it follows that $\boldsymbol{O}_{3^{\prime}}(\mathfrak{C})=1$, where $\mathfrak{E}=\boldsymbol{C}\left(\mathfrak{\mho}_{1}\right)$. Let $\Re=\boldsymbol{O}_{3}(\mathfrak{F}) / \mathfrak{\mho}_{1}$. Thus, $\mathfrak{\Omega} *\langle F\rangle$ is faithfully represented on $\boldsymbol{Z}(\Re)$ for each $F$ in $\mathfrak{F}_{0}-\mathfrak{F}_{1}$. But $[\Re, F] \subseteq$ $\left\langle 3, \widetilde{F}_{1}\right\rangle / \widetilde{\mho}_{1}$, so $\mathfrak{Q}^{*}$ centralizes a subgroup of $O_{3}(\Re)$ of index 9 .

Suppose (9.19) holds and $O_{3}(\mathbb{E}) \cap C(I)$ is noncyclic, where $I$ is the involution of $\mathfrak{Z}^{*}$. In this case, since $\mathfrak{\mho}_{0}$ centralizes $I$ and $\boldsymbol{O}_{3}(\mathfrak{E}) \cap \mathfrak{F}_{0}=$ $\mathfrak{F}_{1}$, it follows that $\mathfrak{C} \cap \mathbb{M}$ contains a subgroup of order 27 and exponent 3. Since every element of $\mathbb{S S}$ of order 3 centralizes an element of $\mathscr{E}(3)$, it follows that a $S_{3}$-subgroup of $\mathfrak{M}$ is nonabelian of order 27 and the width of $\boldsymbol{O}_{2}(\mathfrak{M})$ is 3 . Since $\left|O_{3}(\mathfrak{E}): O_{3}(\mathfrak{E}) \cap C(I)\right|=9$, it follows that $\left|\boldsymbol{O}_{3}(\mathbb{E})\right| \leqq 3^{4}$. Since $\boldsymbol{O}_{3}(\mathbb{E}) \cap C(I)$ is assumed noncyclic, and since $m\left(\boldsymbol{Z}\left(\boldsymbol{O}_{3}(\mathfrak{E})\right) \geqq 3\right.$, it follows that $\boldsymbol{O}_{3}(\mathfrak{E})$ is elementary of order $3^{4}$. Since $\mathfrak{\Omega}^{*} \subseteq \mathfrak{E}$, and since $\langle I\rangle=\boldsymbol{O}_{2}(\mathfrak{M}) \cap \boldsymbol{C}\left(\mathfrak{F}_{0}\right)$, it follows that $\boldsymbol{O}_{3}(\mathfrak{E})$ is of index 3 in $\mathfrak{P}^{*}$. Hence, $|\mathfrak{P}|=3^{6}$, since $\mathfrak{P}^{*}$ is of index 3 in some $S_{3^{-}}$ subgroup of $\$ 3$.

Since $\left|\mathfrak{P}^{*}\right|=3^{5}$, we have $\mathfrak{P}^{*}=\boldsymbol{O}_{3}(\mathfrak{S}) \mathfrak{F}_{0}$.

We argue that $C_{\mathfrak{P}^{*}}(F)$ is of index 9 in $\mathfrak{P}^{*}$ for every $F$ in $\widetilde{F}_{0}-\widetilde{F}_{1}$. This assertion is equivalent to the assertion that $\boldsymbol{O}_{3}(\mathbb{E}) \cap \boldsymbol{C}(F)$ is of order 9 , since $\mathfrak{P}^{*}=\boldsymbol{O}_{3}(\mathfrak{E})\langle F\rangle$. Now $\boldsymbol{O}_{3}(\mathfrak{E})=\mathfrak{U}_{1} \times \mathfrak{U}_{2}$, where $\mathfrak{U}_{1}=$ $C(I) \cap \boldsymbol{O}_{3}(\mathfrak{E}), \mathfrak{U}_{2}$ is inverted by $I$, and $\left|\mathfrak{U}_{1}\right|=\left|\mathfrak{U}_{2}\right|=9$. Since $\mathfrak{U}_{i} \triangleleft \mathfrak{P}^{*} \mathfrak{Q}^{*}$, $i=1,2$, we must show that $F$ does not centralize either $\mathfrak{U}_{1}$ or $\mathfrak{U}_{2}$. It is obvious that $F$ does not centralize $\mathfrak{u}_{2}$. If $F$ centralizes $\mathfrak{U}_{1}$, then $\left\langle\mathfrak{U}_{1}, F\right\rangle$ is elementary of order 27 and is contained in $\mathfrak{M}$, whereas we already know that $S_{3}$-subgroups of $\mathfrak{M}$ are nonabelian of order 27 . So 


\section{$\left|\mathfrak{P}^{*}: C_{\mathfrak{P}^{*}}(F)\right|=9$.}

Since $C_{\mathfrak{P}^{*}}(F)$ is of index 9 in $\mathfrak{P}^{*}$ for every $F$ in $\mathfrak{\mho}_{0}-\widetilde{F}_{1}$, it follows that $O_{3}(\mathbb{E})$ char $\mathfrak{B}^{*}$. Thus, $N\left(O_{3}(\mathfrak{E})\right)$ contains a $S_{3}$-subgroup of $\mathbb{S}$ and $S_{2,3}$-subgroup of $N\left(O_{3}(\mathbb{E})\right)$ are not 3 -closed. This implies that if $\tilde{\mathfrak{R}}$ is a $S_{3}$-subgroup of $N\left(O_{3}(\mathbb{E})\right)$, then $\boldsymbol{O}_{3}(\mathbb{E})$ is not characteristic in $\tilde{\mathfrak{R}}$. More explicitly, $N\left(\boldsymbol{O}_{3}(\mathfrak{S})\right) \cap N(\tilde{\mathfrak{P}})$ does not contain a noncyclic abelian subgroup of order 8, while $\boldsymbol{N}(\tilde{\mathfrak{R}})$ does. Let $\mathfrak{A}$ be an elementary subgroup of $\mathfrak{P}$ of order $3^{4}$ with $\mathfrak{Y} \neq \boldsymbol{O}_{3}(\mathfrak{F})$. If $\mathfrak{U} \cap \boldsymbol{O}_{3}(\mathfrak{S})$ is of order 9 , then $\tilde{\mathfrak{R}}=$ $\mathfrak{U} \boldsymbol{O}_{3}(\mathfrak{S})$ and $\boldsymbol{Z}(\tilde{\mathfrak{P}})$ is not cyclic. Hence, $\mathfrak{A} \cap \boldsymbol{O}_{3}(\mathfrak{S})$ is of order 27 . Hence, $\tilde{\mathfrak{P}}=\mathfrak{P} * \mathfrak{A}$, and it follows that $N\left(\mathrm{O}_{3}(\mathfrak{S})\right) \cap C(I)$ contains $S_{3}$-subgroups of order $3^{4}$. Furthermore, every subgroup of $\tilde{\mathfrak{R}}$ of order 3 centralizes an element of $\mathscr{E}(3)$. Since the width of $\boldsymbol{O}_{2}(\mathfrak{M})$ is 3 , it follows that a $S_{3}$ subgroup of $\mathfrak{M}$ is of the shape $Z_{3} \int Z_{3}$. But we have already shown that $S_{3}$-subgroups of $\mathfrak{M}$ are of order 27 .

Suppose (9.19) holds and $\boldsymbol{O}_{3}(\mathbb{E}) \cap C(I)$ is cyclic. Since $S_{3}$-subgroups of $\mathfrak{M}$ are of exponent 3 or 9 , it follows that $\left|O_{3}(\mathbb{E}) \cap C(I)\right|=3$ or 9 . Hence, $\left|\boldsymbol{O}_{3}(\mathfrak{C})\right| \leqq 3^{4}$, so $\boldsymbol{O}_{3}(\mathfrak{E})$ is abelian. Hence, $\mathfrak{P}^{*}=\boldsymbol{O}_{3}(\mathfrak{E}) \widetilde{\mho}_{0}$. Since elements of $\widetilde{F}_{0}-\mathfrak{F}_{1}$ have quadratic minimal polynomial of $\boldsymbol{O}_{3}(\mathfrak{E})$, it follows that $\sigma^{1}\left(\mathfrak{P}^{*}\right)=\sigma^{1}\left(\boldsymbol{O}_{3}(\mathfrak{E})\right)=\sigma^{1}\left(\boldsymbol{O}_{3}(\mathfrak{E}) \cap \boldsymbol{C}(I)\right)$. Hence, $\sigma^{1}\left(\mathfrak{P}^{*}\right)=1$, since otherwise $\sigma^{1}\left(\mathfrak{B}^{*}\right)$ is conjugate to 3 , against $(9.22)$. Hence, $\boldsymbol{O}_{3}(\mathbb{C})$ is elementary of order 27.

Since $\left|O_{3}(\mathfrak{E})\right|=27$, we get $\left|\mathfrak{P}^{*}\right|=3^{4},|\mathfrak{P}|=3^{5}$. Since (9.19) holds, $\mathfrak{D}$ is of type $(4,2)$ and $\mathfrak{Q}$ normalizes $\mathfrak{B}$. Let $\mathfrak{Q}=\tilde{\mathfrak{D}} \cap C(3)$. Thus, $\widetilde{\mathfrak{Q}}$ is cyclic of order 4 , by Lemma 9.12 (ii). Also, the involution $Q$ of $\widetilde{\mathfrak{Q}}$ inverts $\mathfrak{F} / \mathbb{Z}$, so inverts $\mathfrak{P} / \mathfrak{Z}$. Hence, $\mathfrak{P} / \mathbb{Z}$ is elementary of order $3^{4}$

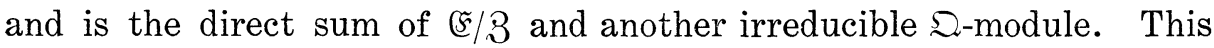
implies that $\mathfrak{P}$ is of exponent 3 and is extra special. Thus, for each $P$ in $\mathfrak{P}, 3$ char $C_{\mathfrak{P}}(P)$. This implies that 3 is weakly closed in $\mathfrak{P}$. But turning back to $\mathfrak{S}$, it follows that $\mathfrak{\Omega}^{*}$ does not normalize 3 , so 3 is not weakly closed in $\mathfrak{B}$. This contradiction shows that (9.19) does not hold.

Suppose (9.20) holds. By (9.24) it follows that $\mathfrak{F}_{0} \subseteq \mathfrak{M}$. Hence, the width of $\boldsymbol{O}_{2}(\mathfrak{M})$ is four. Hence, $\boldsymbol{C}_{\mathfrak{P}_{0}}(I)=\mathfrak{\mho}_{0}$. Thus, $\boldsymbol{C}_{\mathfrak{\Re}_{1}}(I)$ contains a $S_{3}$-subgroup $\tilde{\mathfrak{P}}$ which is a nonabelian group of order 27 and exponent 3. This is not the case, since $C(P) \cap \boldsymbol{O}_{2}(\mathfrak{M})$ contains no four-subgroup for any element $P$ of $\tilde{R}^{\sharp}$.

Suppose (9.21) holds. By (9.24) and $(9.21)^{\prime}$, it follows that $S_{3^{-}}$ subgroups of $\mathfrak{M}$ are of order at least $3^{4}$. Hence, the width of $\boldsymbol{O}_{2}(\mathfrak{M})$ is four, and $C(I) \cap \mathfrak{P}_{0}$ contains no subgroup of order 27 and exponent 3 , and of course $C(I) \cap \mathfrak{P}_{0}$ is of exponent 9. This is absurd, since $S_{3}$ subgroups of Aut $\left(\boldsymbol{O}_{2}(\mathfrak{M})\right)$ contain subgroups of index and exponent 3 . This completes the proof of this lemma.

LEMmA 9.14. $\quad \boldsymbol{N}(\boldsymbol{J}(\mathfrak{P}))$ does not contain a noncyclic abelian subgroup 
of order 8.

Proof. First, suppose that $\boldsymbol{J}(\mathfrak{P})$ is not elementary. Then $\mathfrak{B}=$ $\boldsymbol{Z}(\boldsymbol{J}(\mathfrak{P})) \cap \boldsymbol{D}(\boldsymbol{J}(\mathfrak{P})) \neq 1$. If $\mathfrak{W}$ is cyclic, then $\Omega_{1}(\mathfrak{W})=3$ char $N(\boldsymbol{J}(\mathfrak{P}))$, so $N(J(\mathfrak{P})) \subseteq \mathfrak{N}$, and this lemma follows from Lemma 9.13. We may assume that $\mathfrak{W}$ is noncyclic. Let $\mathfrak{W}_{1}$ be a noncyclic elementary subgroup of order 9 . We will show that $\mathfrak{W}_{1} \in \mathscr{E}(3)$. Choose $\mathfrak{Q} \in \boldsymbol{U}\left(\mathfrak{W}_{1} ; 3^{\prime}\right)$, minimal subject to $\left[\mathfrak{Q}, \mathfrak{W}_{1}\right] \neq 1$. Let $\mathfrak{W}_{0}=C_{\mathfrak{W}_{1}}(\mathfrak{Q})$ so that $\left|\mathfrak{W}_{0}\right|=3$. Let $\mathbb{E}=\boldsymbol{C}\left(\mathfrak{W}_{0}\right)$, and let $\mathfrak{P}^{*}$ be a $S_{3}$-subgroup of $\sqrt{5}$ which contains $\boldsymbol{J}(\mathfrak{P})$. Hence, $\boldsymbol{J}(\mathfrak{P})=\boldsymbol{J}\left(\mathfrak{P}^{*}\right)$. Let $\mathfrak{P}=\mathfrak{P}^{G}$ be a $S_{3}$-subgroup of $(5)$ which contains $\mathfrak{P}^{*}$.

Since $\mathbb{E}$ contains an element of $\mathscr{S}_{\mathrm{v}^{\prime} z_{3}}(\mathfrak{P})$, it follows that $\boldsymbol{O}_{3^{\prime}}(\mathbb{S})=1$. Since $\mathfrak{W}_{1} \subseteq \boldsymbol{Z}(\boldsymbol{J}(\mathfrak{P}))=\boldsymbol{Z}\left(\boldsymbol{J}\left(\mathfrak{B}^{*}\right)\right)$, we have $\left[\boldsymbol{O}_{3}(\mathfrak{S}), \mathfrak{W}_{1}\right] \subseteq \boldsymbol{J}(\mathfrak{P})$ and $\left[\boldsymbol{O}_{3}(\mathfrak{S})\right.$, $\left.\mathfrak{W}_{1}, \mathfrak{W}_{1}\right]=1$. It follows that $\mathfrak{Q}$ is a quaternion group. Let $\mathfrak{B}=\left(\mathfrak{马}^{G}\right)^{\mathfrak{E}}$. Thus, $\mathfrak{B}$ is a normal elementary 3 -subgroup of $\mathfrak{E}$, and by Lemma 5.10 , $\mathfrak{B}$ is 3-reducible in $\mathfrak{c}$. It is a straightforward consequence of Lemma 5.2 that $\mathfrak{B} \subseteq \boldsymbol{J}\left(\mathfrak{B}^{*}\right)$. Thus, $\mathfrak{O}$ centralizes $\mathfrak{B}$, as $\mathfrak{W}_{1}$ centralizes $\mathfrak{B}$. Thus, it follows that $\mathfrak{B}_{1} \nsubseteq \boldsymbol{O}_{3}\left(\mathfrak{N}_{1}\right)$, where $\mathfrak{R}_{1}$ is a $S_{2,3}$-subgroup of $\mathfrak{R}^{G}$ which contains $\mathfrak{P}^{G}$. By Lemma 9.12, $\left|\mathfrak{P}^{G}: \boldsymbol{O}_{3}\left(\mathfrak{N}_{1}\right)\right| \leqq 3$. Thus, $\mathfrak{B}_{1} \nsubseteq \boldsymbol{D}\left(\mathfrak{P}^{G}\right)$. This is absurd, since $\mathfrak{W}_{\perp} \subseteq \boldsymbol{D}(\boldsymbol{J}(\mathfrak{F}))$, and $\boldsymbol{J}(\mathfrak{P})=\boldsymbol{J}\left(\mathfrak{P}^{*}\right)=\boldsymbol{J}\left(\mathfrak{F}^{G}\right)$.

It is an immediate consequence of the preceding argument and Lemmas 7.4 and 5.38 that if $\boldsymbol{D}(\boldsymbol{J}(\mathfrak{B})) \neq 1$, then this lemma holds.

Assume now that $\boldsymbol{J}(\mathfrak{F})$ is elementary. To complete the proof of the lemma, it suffices to show that each subgroup of $\boldsymbol{J}(\mathfrak{P})$ of order 9 is in $\mathscr{E}(3)$. Suppose false, and $\mathfrak{W} \subseteq \boldsymbol{J}(\mathfrak{P}),|\mathfrak{W}|=9, \mathfrak{B} \notin \mathscr{E}(3)$. Let $\mathfrak{I}$ be an element of $И\left(\mathfrak{W} ; 3^{\prime}\right)$ minimal subject to $[\mathfrak{W}, \mathfrak{I}] \neq 1$. Let $\mathfrak{W}_{0}=$ $\mathfrak{W} \cap \boldsymbol{C}(\mathfrak{I})$, so that $\left|\mathfrak{W}_{0}\right|=3$.

Let $\mathbb{E}=\boldsymbol{C}\left(\mathfrak{W}_{0}\right) \supseteqq\langle\boldsymbol{J}(\mathfrak{P}), \mathfrak{I}\rangle$. Let $\mathfrak{P}^{*}$ be a $S_{3}$-subgroup of $\mathfrak{E}$ which contains $\boldsymbol{J}(\mathfrak{B})$, and let $\mathfrak{P}^{G}$ be a $S_{3}$-subgroup of $\mathbb{S}$ which contains $\mathfrak{P}^{*}$. Hence, $\boldsymbol{J}(\mathfrak{P})=\boldsymbol{J}\left(\mathfrak{P}^{G}\right)$. Since $\boldsymbol{J}\left(\mathfrak{P}^{G}\right)=\boldsymbol{J}(\mathfrak{P})^{G}$, we get that $G \in \boldsymbol{N}(\boldsymbol{J}(\mathfrak{P}))$. Replacing $\mathfrak{W}$ by $\mathfrak{W}^{G^{-1}}$ and $\mathfrak{I}$ by $\mathfrak{I}^{G^{-1}}$, we assume without loss of generality that $\mathfrak{S}^{*} \leqq \mathfrak{s}$.

Since $\mathfrak{S}^{*}$ contains an element of $\mathscr{S} \mathscr{C} \mathscr{N}_{3}(\mathfrak{P})$, it follows that $\boldsymbol{O}_{3^{\prime}}(\mathfrak{C})=$ 1. Hence, $3 \subseteq \boldsymbol{O}_{3}(\mathfrak{E})$. Let $\mathfrak{B}=\mathfrak{Z}^{\mathfrak{E}}$, so that $\mathfrak{B}$ is a normal elementary subgroup of $\mathfrak{\complement}$. Since $\mathfrak{B}$ is 3 -reducible in $\mathfrak{E}$, it follows that $\mathfrak{B} \subseteq \boldsymbol{J}(\mathfrak{P})$. Hence, $\mathfrak{I}$ centralizes $\mathfrak{B}$. In particular, $\mathfrak{I}$ centralizes 3 .

Let $\widetilde{\widetilde{S}}=\boldsymbol{J}(\mathfrak{P}) \cap \boldsymbol{O}_{3}(\mathfrak{S})$. Thus, $\mathfrak{W} \nsubseteq \widetilde{\widetilde{F}}$, and $\boldsymbol{J}(\mathfrak{P}) / \widetilde{\Im}$ acts faithfully on $\boldsymbol{O}_{3,3^{\prime}}(\mathfrak{S}) / \boldsymbol{O}_{3}(\mathfrak{S})$. Let $\mathfrak{R}=\left[\boldsymbol{O}_{3,3^{\prime}}(\mathfrak{S}), \boldsymbol{J}(\mathfrak{P})\right] \boldsymbol{O}_{3}(\mathfrak{S})$. Since

$$
\left[\boldsymbol{O}_{3}(\mathfrak{S}), J(\mathfrak{P}), J(\mathfrak{P})\right]=1,
$$

it follows that $\bar{\Re}=\Re / O_{3}(\mathfrak{C})$ is a 2 -group, and that $J(\Re)$ centralizes every characteristic abelian subgroup of $\bar{\Re}$. Since $J(\mathfrak{P})$ centralizes $\mathfrak{B}$, so does $\Re$. By Lemma 9.12 (ii), $\Re$ contains no four-group. So $\Re$ is a quaternion group and $\boldsymbol{J}(\mathfrak{P}) / \widetilde{\Im}$ is of order 3 , whence $\boldsymbol{J}(\mathfrak{P})=\widetilde{\Im} \mathfrak{W}$, and so $\Re=$ 


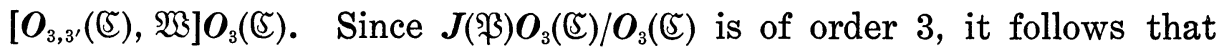
$\boldsymbol{J}(\mathfrak{P}) \subseteq \boldsymbol{O}_{3,3^{\prime}, 3}(\mathfrak{E})$, and so $\mathfrak{I} \subseteq \boldsymbol{O}_{3,3^{\prime}}(\mathfrak{E})$, whence $\mathfrak{T} \subseteq \Re$, and so $\mathfrak{R}=\mathfrak{T} \boldsymbol{O}_{3}(\mathfrak{F})$, and $\mathfrak{I} \cong \overline{\mathfrak{R}}$ is a quaternion group. Note that $\mathfrak{T}$ is permutable with $\mathfrak{P}^{*}$, as $\mathfrak{P}^{*}$ normalizes $\left[\boldsymbol{O}_{3,3^{\prime}}(\mathfrak{E}), \boldsymbol{J}(\mathfrak{P})\right]$.

Enlarge $\mathfrak{P}^{*} \mathfrak{I}$ to a $S_{2,3^{-}}$-subgroup $\mathfrak{N}_{0}$ of $\mathfrak{R}$, and let $\mathfrak{R}_{1}=\boldsymbol{O}^{\prime \prime}\left(\mathfrak{R}_{0}\right)$. Thus, $\mathfrak{R}_{1}=\mathfrak{I} \tilde{\mathfrak{P}}$, where $\tilde{\mathfrak{P}}=\mathfrak{S}^{N}$ for some $N$ in $\mathfrak{R}$. Since $\boldsymbol{J}(\mathfrak{P})=\boldsymbol{J}\left(\mathfrak{P}^{N}\right)=$ $\boldsymbol{J}(\mathfrak{P})^{N}$, replacing $\mathfrak{B}$ by $\mathfrak{W}^{N^{-1}}$ and $\mathfrak{T}$ by $\mathfrak{T}^{N^{-1}}$, we assume without loss of generality that $\tilde{\mathfrak{P}}=\mathfrak{P}$ is permutable with $\mathfrak{T}$.

Let $\mathfrak{W}_{1}$ be a subgroup of $\mathfrak{W}$ of order 3 different from $\mathfrak{W}_{0}$. Let $\mathfrak{P}_{0}=\boldsymbol{O}_{3}\left(\mathfrak{N}_{1}\right)$. Thus, $\mathfrak{P}=\mathfrak{P}_{0} \mathfrak{W}_{1}$ and $\mathfrak{W}_{1} \mathfrak{T}$ is a complement to $\mathfrak{P}_{0}$ in $\mathfrak{R}_{1}$. Let $I$ be the involution of $\mathfrak{I}$, let $T$ be an element of $\mathfrak{I}$ of order 4 , let $\Re=J(\Re) \cap \mathfrak{P}_{0}$, and let $\mathfrak{L}=\left\langle\Re, \Re^{T}\right\rangle$. Since $T^{2}=I$ normalizes $\mathfrak{P}$, and $J(\mathfrak{P})$ char $\mathfrak{P}$, it follows that $T^{2}$ normalizes $\Re$. Hence, $T$ normalizes

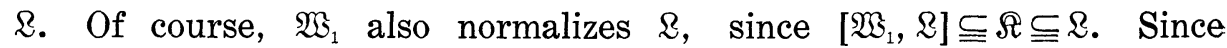
$\mathfrak{R} \triangleleft \mathfrak{P}_{0}$, it follows that $\mathfrak{R} \triangleleft \mathfrak{P}_{0}$, so $\mathfrak{L} \triangleleft \mathfrak{R}_{1}$. Since

$$
\mathbb{L}^{\prime}=\left[\Re, \Re^{T}\right] \cong \Re \cap \Re^{T} \subseteq \Re \cong J(\Re),
$$

$\boldsymbol{J}(\mathfrak{P})$ centralizes $\mathbb{R}^{\prime}$. Since $\mathfrak{W}_{1} \subseteq J(\mathfrak{P})$, it follows that $\mathfrak{I}$ centralizes $\mathbb{R}^{\prime}$. Hence, $\mathbb{R}^{\prime} \subseteq 3$, as otherwise $I$ centralizes an element of $\mathscr{C}(3)$.

Clearly, $\mathcal{Z}$ is of exponent 3 , being of class at most 2 and being generated by its elementary subgroups. The definition of $\boldsymbol{J}(\mathfrak{P})$ forces $\Re=C_{\mathfrak{\Omega}}(\Re)$. Hence, $\boldsymbol{Z}(\mathfrak{\Omega})=3=\mathbb{R}^{\prime}$, so that $\mathfrak{R}$ is extra special, while $\Re \in \mathscr{S}_{\text {cor }}(\mathbb{R})$. The width of $\mathbb{R}$ is at least 2 , since otherwise, Hypothesis 9.1 would be satisfied.

Now $I$ centralizes 3 and normalizes $\Re$. We argue that $I$ inverts $\Re / 3$. Suppose false and $\widetilde{F}$ is a subgroup of $\Re$ of order 9 which contains 3 and is centralized by $I$. Since $\boldsymbol{A}_{\mathfrak{N}}(\tilde{\mathscr{C}})=\boldsymbol{A}(\tilde{\mathscr{C}})$, where $\tilde{\mathscr{C}}: \Re \supset 3 \supset 1$, it follows from Lemma 5.5 that $\widetilde{F} \in \mathscr{E}(3)$. Thus, $C(I)$ is nonsolvable by Lemmas 7.4 and 5.38. This contradiction shows that $I$ inverts $\Re / 3$. Hence $I$ inverts $\mathfrak{L} / \mathbb{Z}$.

We next show that $\mathfrak{I}$ centralizes $\mathfrak{P}_{0} / \mathbb{R}$. This is clear, since $\left[\mathfrak{P}_{0}, \mathfrak{W}_{1}\right] \subseteq \Re \subseteq \mathbb{R}$, so that $\mathfrak{W}_{1}$ centralizes $\mathfrak{P}_{0} / \mathbb{R}$.

Since $I$ inverts $\mathbb{L} / 3$, it follows that if $\widetilde{\mathfrak{W}}$ is any subgroup of $J(\mathfrak{P})$

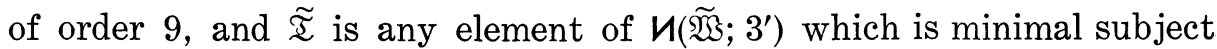
to $[\widetilde{\mathfrak{W}}, \widetilde{\mathfrak{I}}] \neq 1$, then $\widetilde{\mathfrak{I}}$ is a quaternion group and $\widetilde{\mathfrak{W}} \cap \boldsymbol{C}(\widetilde{\mathfrak{T}}) \sim 3$. In particular, $\mathfrak{B} \in \mathscr{D}$.

Let $\mathfrak{M}$ be the subgroup given by Lemma 7.5 which contains a $S_{2}$-subgroup of $C(I)$ and contains $\mathfrak{W}$. Then Lemma 9.12 (ii) implies that $\boldsymbol{O}_{2}(\mathfrak{M})$ is extra special, and that $\langle I\rangle=\boldsymbol{O}_{2}(\mathfrak{M})^{\prime}$. Hence, $\boldsymbol{C}_{\mathfrak{R}_{1}}(I) \subseteq \mathfrak{M}$.

If the width of $\boldsymbol{O}_{2}(\mathfrak{M})$ is 2 , then $\mathfrak{M}=\boldsymbol{C}_{\mathfrak{S}}(I)$ is a $S_{3}$-subgroup of $C_{\mathfrak{R}_{1}}(I)$ so $\mathfrak{Z}=\mathfrak{P}_{0}$ is extra special. Since $\mathfrak{P}_{0}=\boldsymbol{O}_{3}(\mathfrak{R})$, it follows that Hypothesis 9.2 is satisfied, an excluded case. Hence, the width of $\boldsymbol{O}_{2}(\mathfrak{M})$ is 3 or 4 . On the other hand, every element of $\left(\boldsymbol{C}(I) \cap \boldsymbol{O}_{3}\left(\mathfrak{N}_{1}\right)\right) \mathfrak{W}$ of order 3. 
centralizes an element of $\mathscr{E}(3)$, so $C_{\mathfrak{N}_{1}}(I)$ contains no subgroup of exponent 3 and order 27. Hence, $C_{\mathfrak{F}_{0}}(I)$ is cyclic of order 3 or 9 . If $C_{\mathfrak{F}_{0}}(I)=3$, Hypothesis 9.2 is satisfied, an excluded case. Hence, $\boldsymbol{C}_{\mathfrak{F}_{0}}(I)$ is cyclic of order 9 . This means that if $\mathfrak{W}_{1}$ is any subgroup of $\mathfrak{W}$ of order 3 such that $\boldsymbol{C}\left(\mathfrak{W}_{1}\right) \cap \boldsymbol{O}_{2}(\mathfrak{M}) \supset\langle I\rangle$, then $\mathfrak{B}_{1}=\left\langle P^{3}\right\rangle$ for some $P$ in $\mathfrak{M}$. This is absurd, since we get that $\mathfrak{B} \subseteq \Omega^{1}(\tilde{\mathfrak{P}})$ for some $S_{3}$-subgroup $\tilde{\mathfrak{R}}$ of $\mathfrak{M}$, while $\tilde{\mathfrak{P}}$ is isomorphic to a subgroup of $Z_{3} \times\left(Z_{3} \int Z_{3}\right)$. The proof is complete.

LEMMA 9.15. If $\mathfrak{A} \in \mathscr{E}(3)$ and $\mathfrak{B}$ is a noncyclic abelian subgroup of order 8 , then $\langle\mathfrak{U}, \mathfrak{B}\rangle$ is nonsolvable.

Proof. Suppose false. Let $\mathscr{S}$ be the set of all 2, 3-subgroups $\mathfrak{S}$ of (3) such that

(i) $\subseteq$ contains an element of $\mathscr{E}(3)$.

(ii) $\mathfrak{S} / \boldsymbol{O}_{3}(\mathfrak{S})$ satisfies the hypothesis of Lemma 5.41 . Thus, $\mathscr{S} \neq \varnothing$.

If $\mathfrak{S}_{1}$ and $\mathfrak{S}_{2}$ are elements of $\mathscr{S}$, we say that $\mathfrak{S}_{1} \ll \mathfrak{S}_{2}$ if and only if either $\left|\mathfrak{S}_{1}\right|_{3}<\left|\mathfrak{S}_{2}\right|_{3}$ or $\mathfrak{S}_{1}=\mathfrak{S}_{2}$.

Let $\subseteq$ be a maximal element of $\mathscr{S}$ under $\ll$. Let $\mathfrak{S}_{p}$ be a $S_{p^{-}}$ subgroup of $\mathfrak{S}, p=2,3$. Since $\mathfrak{S}$ contains an element of $\mathscr{E}(3)$, it follows from Lemma 9.11 (ii) that $O_{2}(S)=1$.

Replacing $\subseteq$ by a conjugate if necessary, we assume that $\mathfrak{S}_{3} \subseteq \mathfrak{P}$. By Lemma 5.41, $\subseteq$ has 2-length 1 . If $\mathfrak{S}_{2}^{\prime}=1$, then

$$
\mathfrak{S}=C_{\Im}\left(Z\left(\Im_{3}\right)\right) \cdot N_{\Im}\left(J\left(\Im_{3}\right)\right)
$$

by Theorem 1 of [43]. Since $\boldsymbol{Z}(\mathfrak{\beta}) \subseteq Z\left(\Im_{3}\right), S_{2}$-subgroups of $\boldsymbol{C}_{\Im}\left(\boldsymbol{Z}\left(\mathfrak{S}_{3}\right)\right)$ are cyclic. Thus, $C_{\Im}\left(Z\left(\Im_{3}\right)\right) \cong N_{\Im}\left(\Im_{3}\right) \subseteq N_{\Im}\left(J\left(\Im_{3}\right)\right)$, so $J\left(\Im_{3}\right) \triangleleft \Im_{\text {. }}$ Maximality of $\subseteq$ forces $\mathfrak{S}_{3}=\mathfrak{P}$, against Lemma 9.14. Hence, $\mathfrak{S}_{2}^{\prime} \neq 1$.

Suppose $\mathfrak{S}_{2}$ is extra special of width at least 2. By Lemma 5.52, it follows that $\subseteq=C_{\Im}\left(Z\left(O_{3}(\subseteq)\right)\right) N_{\Im}\left(J\left(\Im_{3}\right)\right)$. Thus, maximality of $\subseteq$ together with Lemmas 9.13 and 9.14 imply that neither $C_{\Im}\left(Z\left(O_{3}(\Im)\right)\right)$ nor $N_{\Im}\left(J\left(\mathscr{S}_{3}\right)\right)$ contains a noncyclic abelian subgroup of order 8 . Let $\mathfrak{I}_{0}=C_{\overleftarrow{S}}\left(Z\left(O_{3}(\mathfrak{S})\right)\right) \cap \mathfrak{S}_{2}, \mathfrak{I}_{1}=N\left(J\left(\mathfrak{S}_{3}\right)\right) \cap \mathfrak{S}_{2}$.

Since $\mathfrak{S}_{2}=\mathfrak{I}_{0} \mathfrak{I}_{1}$ and $\mathfrak{I}_{i}$ has no noncyclic abelian subgroup of order 8, the width of $\mathfrak{S}_{2}$ is 2 , and $4 \leqq\left|\mathfrak{I}_{i}\right| \leqq 8, i=0,1$.

Suppose $\mathfrak{S}_{3} \cdot C_{\Im}\left(\boldsymbol{Z}\left(\boldsymbol{O}_{3}(\mathfrak{S})\right)\right)$ is 3 -closed. Then $\mathfrak{I}_{0}$ normalizes $\mathfrak{S}_{3}$, so normalizes $J\left(\Im_{3}\right)$. This yields $\mathfrak{I}_{0} \subseteq \mathfrak{I}_{1}$, which is not the case. Thus, $\mathfrak{S}_{3} \cdot C_{\mathfrak{S}}\left(Z\left(O_{3}(\mathfrak{S})\right)\right)$ is not 3-closed. Since $\mathfrak{I}_{0} \triangleleft \mathfrak{S}_{2}$, it follows that $\mathfrak{I}_{0}$ is a quaternion group. Suppose $N_{\Im}\left(J\left(\Im_{3}\right)\right)$ is 3-closed. Since $Z\left(\Im_{3}\right) \subseteq$ $\boldsymbol{Z}\left(\boldsymbol{O}_{3}(\Im)\right)$, it follows that $\boldsymbol{Z}\left(\Im_{3}\right) \triangleleft \Im$. Maximality of $|\Im|_{3}$ forces $\Im_{3}=\mathfrak{P}$. This violates Lemma 9.13. Thus, $N_{\circlearrowleft}\left(J\left(\Im_{3}\right)\right)$ is not 3-closed. Since

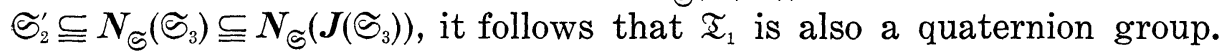
Since $l_{2}(\mathfrak{S})=1, \mathfrak{S}_{2}$ is the central product of $\mathfrak{I}_{0}$ and $\mathfrak{I}_{1}$. 
Let $\mathfrak{P}=\mathfrak{P}_{1}, \cdots, \mathfrak{P}_{r}$ be all the $S_{3}$-subgroups of (S) which contain $\mathfrak{S}_{3}$, and let $\bigotimes_{i}=\Omega_{1}\left(\boldsymbol{Z}\left(\mathfrak{P}_{i}\right)\right)$. Thus, $Z_{i} \subseteq \boldsymbol{Z}\left(\boldsymbol{O}_{3}(\mathfrak{S})\right)$ for all $i$, so that $\mathfrak{I}_{0}$ centralizes each $3_{i}$. Let $\langle T\rangle=\mathfrak{I}_{0} \cap \mathfrak{I}_{1}$ so that $T$ is an involution which centralizes each $3_{i}$. Also, $\mathfrak{I}_{0} \leqq C\left(3_{i}\right)$ for each $i$, so for each $i, \mathfrak{I}_{1} \nsubseteq \boldsymbol{C}\left(\mathfrak{Z}_{i}\right)$.

Suppose $\mathfrak{S}_{3}=\mathfrak{P}$. Since $\mathfrak{S}_{2}^{\prime}=\mathfrak{I}_{j}^{\prime}$ centralizes $Z\left(\boldsymbol{O}_{3}(\mathfrak{S})\right)$, it follows that $3=\Omega_{1}\left(\boldsymbol{Z}\left(\boldsymbol{O}_{3}(\mathfrak{S})\right)\right.$; otherwise, $\mathfrak{S}_{2}^{\prime}$ centralizes an element of $\mathscr{U}(3)$. Hence, $3 \triangleleft \mathfrak{S}$, against Lemma 9.13. We conclude that $\mathfrak{S}_{3} \subset \mathfrak{P}$.

Enlarge $\mathfrak{S}_{3} \mathfrak{I}_{1}$ to a $S_{2,3}$-subgroup of $\boldsymbol{N}\left(\boldsymbol{J}\left(\mathfrak{S}_{3}\right)\right)$ and enlarge this subgroup to a maximal 2 , 3-subgroup $\Omega_{0}$ of $(S)$. Let $\mathbb{R}=\boldsymbol{O}^{3^{\prime}}\left(\mathbb{\Omega}_{0}\right)$. Since $|\mathbb{R}|_{3}>|\mathfrak{S}|_{3}$, it follows that $\mathbb{R}$ contains no noncyclic abelian subgroup of order 8. Since $\mathfrak{R} \supseteqq \boldsymbol{O}^{3}\left(\mathfrak{S}_{3} \mathfrak{I}_{1}\right)=\mathfrak{S}_{3} \mathfrak{T}_{1}$, it follows that $\mathfrak{I}_{1}$ is a $S_{2^{-}}$ subgroup of $\mathbb{L}$. Let $\mathbb{Z}_{3}$ be a $S_{3}$-subgroup of $\mathbb{Q}$ which contains $\mathfrak{S}_{3}$. Thus, $\mathfrak{Z}_{3} \subseteq \mathfrak{P}_{i}$ for some $i$.

Let $\mathfrak{W}$ be the normal closure of $Z_{i}$ in $\mathcal{R}$. Thus, $C_{\mathfrak{Q}}(\mathfrak{W})$ contains $T$. Since $\mathfrak{I}_{0} \subseteq C\left(\Re_{i}\right)$, it follows that $C_{\mathfrak{Q}}(\mathfrak{W}) \cap \mathfrak{I}_{1}=\langle T\rangle$, so $S_{2}$-subgroups of $\boldsymbol{A}_{\mathbb{\Omega}}(\mathfrak{W})$ are four-groups. It follows that $\boldsymbol{J}\left(\mathbb{Q}_{3}\right) \triangleleft \mathbb{Q}$. Hence, $\mathbb{Q}_{3}=\mathfrak{P}_{i}$ and so $T$ centralizes an element of $\mathscr{\mathscr { C }}\left(\mathfrak{P}_{i}\right)$. Thus, by Lemmas 7.1 (i) and 7.4, $C(T)$ is nonsolvable. This contradiction shows that $\mathfrak{S}_{2}$ is not extra special of width $\geqq 2$.

Suppose $\mathfrak{S}_{2}$ is the central product of a quaternion group and a cyclic group of order 4 . If $J\left(\mathfrak{S}_{3}\right) \subseteq O_{3}(\Im)$, then again $\mathfrak{S}_{3}=\mathfrak{P}$ and Lemma 9.14 is violated. Hence, $\boldsymbol{J}\left(\mathfrak{S}_{3}\right) \nsubseteq \boldsymbol{O}_{3}(\mathfrak{S})$. If $\mathfrak{S}_{2}^{\prime}$ centralizes $\boldsymbol{Z}\left(\boldsymbol{O}_{3}\left(\Im_{)}\right)\right.$, then we get $\subseteq=\boldsymbol{C}_{\Im}\left(\boldsymbol{Z}\left(\Im_{3}\right)\right) \boldsymbol{N}_{\Im}\left(\boldsymbol{J}\left(\Im_{3}\right)\right)$, so that either $\boldsymbol{Z}\left(\Im_{3}\right)$ or $J\left(\subseteq_{3}\right)$ is normal in $\subseteq$. Both these possibilities are excluded by Lemmas 9.13 and 9.14 , so we may assume that $\left[\mathfrak{S}_{2}^{\prime}, Z\left(O_{3}(\mathfrak{S})\right)\right]=\mathfrak{W} \neq 1$. Let $\mathfrak{X}$ be a minimal normal subgroup of $\subseteq$ with $\mathfrak{X} \subseteq \mathfrak{M}$. Thus, $\mathfrak{S}_{2}$ is faithfully represented on $\mathfrak{X}$. Since $\left|\mathfrak{S}_{3}: \boldsymbol{O}_{3}\left(\Im_{S}\right)\right|=3$ and $\boldsymbol{J}\left(\mathfrak{S}_{3}\right) \nsubseteq \boldsymbol{O}_{3}\left(\subseteq_{)}\right)$, it follows that elements of $\mathfrak{S}_{3}-\boldsymbol{O}_{3}(\mathfrak{S})$ centralize a hyperplane of $\mathfrak{X}$. This is not the case, since $|\mathfrak{X}|=3^{4}$. Thus, $\mathfrak{S}_{2}$ is not the central product of a quaternion group and a cyclic group of order 4 .

By Lemma 5.41 and maximality of $\mathfrak{\subseteq}$ under $\ll$, it follows that $\mathfrak{S}_{2}$ is either the direct product of a quaternion group and a group of order 2 or $\mathfrak{S}_{2}$ is special with $\left|\mathfrak{S}_{2}^{\prime}\right|=4$. Let $\mathfrak{B}=Z\left(\mathfrak{S}_{2}\right)$, so that in both cases, $\mathfrak{B}$ is a four-group. We will exploit $\mathfrak{B}$ by showing that $\mathfrak{S}_{3}=\mathfrak{P}$, that is, by showing that $\mathfrak{S}_{3}$ is a $S_{3}$-subgroup of (G). Suppose by way of contradiction that $\widetilde{S}_{3} \subset \mathfrak{P}$.

We argue that $\mathfrak{B}$ normalizes $\boldsymbol{J}\left(\mathfrak{S}_{3}\right)$. For if this is not the case, then $\mathfrak{B}$ centralizes $\boldsymbol{Z}\left(\boldsymbol{O}_{3}(\mathfrak{S})\right.$ ), against Lemma 9.12 (ii).

Since $\mathfrak{B}$ normalizes $\boldsymbol{J}\left(\mathfrak{S}_{3}\right)$, we may enlarge $\mathfrak{S}_{3} \mathfrak{B}$ to a $S_{2,3}$-subgroup $\mathcal{L}$ of $N\left(\boldsymbol{J}\left(\mathfrak{S}_{3}\right)\right)$. Since $\mathfrak{S}_{3}$ is not a $S_{3}$-subgroup of $\mathbb{2}, \mathbb{\&}$ does not contain a noncyclic abelian subgroup of order 8 .

Let $\mathfrak{R}_{p}$ be a $S_{p}$-subgroup of $\mathfrak{R}, p=2,3$, with $\mathfrak{B} \subseteq \mathbb{R}_{2}, \mathfrak{S}_{3} \subset \mathbb{Z}_{3}$. 
Case 1. $\boldsymbol{O}_{3}(\mathfrak{S}) \mathfrak{B} / \boldsymbol{O}_{3}(\mathfrak{S}) \subseteq Z\left(\Im / O_{3}(\Im)\right)$.

Let $\widetilde{\Im}_{3}$ be a maximal element of $h_{\mathfrak{L}}(\mathfrak{B} ; 3)$ with $\widetilde{S}_{3} \subseteq \widetilde{\subseteq}_{3}$. Suppose $\widetilde{S}_{3} \subset \widetilde{\mathfrak{S}}_{3}$. Choose $\widetilde{S}_{3}^{*}$ in $\boldsymbol{U}_{\mathfrak{\Omega}}(\mathfrak{B} ; 3)$ so that $\left|\mathfrak{S}_{3}^{*}: \mathfrak{S}_{3}\right|=3$, and let $\mathfrak{B}_{0}=$ $\boldsymbol{C}_{\mathfrak{B}}\left(\mathfrak{S}_{3}^{*} / \mathfrak{S}_{3}\right)$. Hence, $\left[\mathfrak{S}_{3}^{*}, \mathfrak{B}_{0}\right]=\left[\mathfrak{S}_{3}, \mathfrak{B}_{0}\right]$ is normal in $\mathfrak{S}$ and in $\mathfrak{S}_{3}^{*}$. Maximality of $\mathfrak{S}$ in $\mathscr{S}$ forces $\left[\mathfrak{S}_{3}, \mathfrak{B}_{0}\right]=1$, against $\boldsymbol{O}_{2}(\mathfrak{S})=1$. Thus, $\mathfrak{S}_{3}$ is a maximal element of $\boldsymbol{U}_{\mathcal{R}}(\mathfrak{B} ; 3)$. In particular, $\mathbb{Z}$ is not 3-closed. Hence, $\boldsymbol{O}_{3}(\mathbb{R})$ is of index 3 in $\mathfrak{\Omega}_{3}$ and $\boldsymbol{O}_{3}(\mathbb{R}) \subseteq \Im_{3}$. Hence, $\boldsymbol{O}_{3}(\mathbb{R})=\mathfrak{S}_{3}$. If $\mathfrak{B}=\mathfrak{Q}_{2}$, then $\left[\mathfrak{B}, \mathfrak{S}_{3}\right] \triangleleft \mathfrak{Q}$ so that $\langle\mathfrak{S}, \mathfrak{R}\rangle \subseteq N\left(\left[\mathfrak{V}, \mathfrak{S}_{3}\right]\right)$, since $\left[\mathfrak{B}, \mathfrak{S}_{3}\right]=$ $\left[\mathfrak{B}, \boldsymbol{O}_{3}(\mathfrak{S})\right] \neq 1$. This is impossible, so $\mathfrak{B} \subset \mathfrak{R}_{2}$.

Case 1a. $\mathfrak{\Im}_{2}$ is special.

Since $\mathfrak{B} \subseteq \boldsymbol{Z}\left(\mathfrak{S}_{2}\right)$, it follows that $\mathfrak{S}_{3} \mathfrak{B}$ is a maximal subgroup of $\mathfrak{S}$. Thus, $\boldsymbol{O}_{3}(\mathfrak{S}) \mathfrak{S}_{2} / \boldsymbol{O}_{3}(\mathfrak{S}) \mathfrak{B}$ is a chief factor of $\mathfrak{S}$. Let $\mathfrak{B}_{0}$ be a subgroup of $\mathfrak{B}$ of order 2 and let $\mathfrak{S}_{2}^{0}=\boldsymbol{Z}\left(\mathfrak{S}_{2} \bmod \mathfrak{B}_{0}\right)$. Since $\boldsymbol{O}_{3}(S) \mathfrak{B}_{0} \triangleleft \mathfrak{S}, \mathfrak{S}_{3} \mathfrak{S}_{2}^{\prime}$ is a group. Hence, $\mathfrak{S}_{2}^{\prime}=\mathfrak{B}$ or $\mathfrak{S}_{2}^{o}=\mathfrak{S}_{2}$. If $\mathfrak{S}_{2}^{\prime}=\mathfrak{S}_{2}$, then $\mathfrak{S}_{2}^{\prime} \subseteq \mathfrak{B}_{0}$, so that $\mathfrak{S}_{2} / \mathfrak{B}_{0}$ is abelian. This is not the case, since $\mathfrak{S}_{2}^{\prime}=\mathfrak{B}$. Hence, $\mathfrak{S}_{2}^{0}=\mathfrak{B}$, so that $\mathfrak{S}_{2} / \mathfrak{B}_{0}$ is extra special of width $\geqq 2$. It follows from the proof of Lemma 5.52 that $J\left(\mathscr{S}_{3}\right) \triangleleft \subseteq$. Maximality of $\subseteq$ in $\mathscr{S}$ guarantees that $\widetilde{S}_{3}$ is a $S_{3}$-subgroup of 8 , against our assumption that $\mathfrak{S}_{3} \subset \mathfrak{P}$.

Case $1 \mathrm{~b} . \widetilde{S}_{2}$ is the direct product of a quaternion group and a group of order 2 .

Since $\mathbb{R}_{2}$ has no noncyclic abelian subgroup of order 8 , it follows that $\mathfrak{B}$ is a self centralizing subgroup of $\mathbb{R}_{2}$. Hence, $\mathbb{Z}_{2}$ is of maximal class. Let $\tilde{\mathfrak{\Omega}}_{2}=\mathfrak{\Omega}_{2} \cap \boldsymbol{O}_{3,2}(\mathfrak{Q})$. Thus, $\widetilde{\mathfrak{I}}_{2}$ has an automorphism of order 3. Being a subgroup of a group of maximal class, $\tilde{\Omega}_{2}$ is either a quaternion group or a four-group.

Suppose $\widetilde{\Omega}_{2}$ is a quaternion group. In this case, $\mathbb{2} / \boldsymbol{O}_{3}(\mathbb{2}) \cong G L(2,3)$ and $\mathfrak{B}$ normalizes a $S_{3}$-subgroup of $\mathfrak{L}$, against our previous argument. we conclude that $\tilde{\mathbb{R}}_{2}$ is a four-group.

If $\tilde{\mathfrak{L}}_{2}=\mathfrak{B}$, then $\left[\boldsymbol{O}_{3}(\mathfrak{R}), \mathfrak{B}\right] \triangleleft \mathfrak{R}$. But $\boldsymbol{O}_{3}(\mathfrak{R})=\mathfrak{S}_{3}$ and $\left[\mathfrak{S}_{3}, \mathfrak{S}\right]=$ $\left[\boldsymbol{O}_{3}(\mathfrak{S}), \mathfrak{B}\right] \triangleleft \mathfrak{S}$. Hence, $\left[\boldsymbol{O}_{3}(\mathfrak{R}), \mathfrak{B}\right] \triangleleft\langle\mathfrak{Q}, \mathfrak{S}\rangle$ against the maximality of $\mathfrak{S}$ in $\mathscr{S}$. We conclude that $\widetilde{\mathfrak{\Omega}}_{2} \neq \mathfrak{B}$, so that $\mathfrak{R}_{2}$ is a dihedral group of order 8 whose two four-subgroups are $\mathfrak{B}$ and $\tilde{\mathfrak{\Omega}}_{2}$.

Since $\tilde{\Omega}_{2}$ does not centralize $\boldsymbol{Z}\left(\boldsymbol{O}_{3}(\mathbb{R})\right)$, it follows that $\boldsymbol{J}\left(\Omega_{3}\right) \triangleleft \mathbb{\Omega}_{0}$. Hence, $\boldsymbol{J}\left(\mathbb{R}_{3}\right)=\boldsymbol{J}\left(\mathfrak{S}_{3}\right)$, so by construction of $\mathbb{R}$, we conclude that $\mathbb{R}_{3}$ is a $S_{3}$-subgroup of $(5)$. We may therefore assume without loss of generality that $\mathfrak{P}=\mathfrak{\Omega}_{3}$. Hence, $\left|\mathfrak{P}: \mathfrak{S}_{3}\right|=3$.

Let $\mathfrak{X}=\Omega_{1}\left(\boldsymbol{Z}\left(\mathfrak{S}_{3}\right)\right)$. Recalling that $\mathfrak{S}_{3}=\boldsymbol{O}_{3}(\mathbb{R})$, we get $\mathfrak{X} \triangleleft \mathbb{R}$. Since $\widetilde{\mathfrak{\Omega}}_{2}$ does not centralize 3 , we get $3 \subset \mathfrak{X}$. Since $Z(\mathfrak{P})$ is cyclic and $\left|\mathfrak{F}: \mathfrak{S}_{3}\right|=3$, we get $|\mathfrak{X}| \leqq 27$. Hence, $|\mathfrak{X}|=27$ and $\mathfrak{X}$ is the only minimal normal subgroup of $\mathbb{R}$.

Since $\boldsymbol{J}(\mathfrak{P}) \triangleleft \mathfrak{L}$, we get $\mathfrak{X}_{0} \triangleleft \mathfrak{L}$, where $\mathfrak{X}_{0}=\Omega_{1}(\boldsymbol{Z}(\boldsymbol{J}(\mathfrak{P})) \cap \boldsymbol{D}(\boldsymbol{J}(\mathfrak{P})))$, if 
$\boldsymbol{D}(\boldsymbol{J}(\mathfrak{P})) \neq 1$, and $\mathfrak{X}_{0}=\boldsymbol{J}(\mathfrak{P})$ if $\boldsymbol{D}(\boldsymbol{J}(\mathfrak{P}))=1$. If $\left|\mathfrak{X}_{0}\right|>27$, then some element of $\mathfrak{B}^{\sharp}$ centralizes a noncyclic subgroup of $\mathfrak{X}_{0}$. This was shown to be impossible in the proof of Lemma 9.14. Hence, $\left|\mathfrak{X}_{0}\right| \leqq 27$. This implies that $\mathfrak{X}_{0}=\mathfrak{X}$.

Suppose $A, B \in \mathfrak{X}_{0}$ and $A=B^{B}$ for some $G$ in (s). Thus, $\langle\boldsymbol{J}(\mathfrak{P})$, $\left.\boldsymbol{J}\left(\mathfrak{P}^{G^{-1}}\right)\right\rangle \subseteq C(B)$, and we can choose $C$ in $C(B)$ such that $J(\mathfrak{P})^{C}=$ $\boldsymbol{J}\left(\mathfrak{P}^{G^{-1}}\right)$. Hence, $C G=N \in N(\boldsymbol{J}(\mathfrak{P}))$ and $A=B^{G}=B^{C^{-1}} N=B^{N}$. Thus, elements of $\mathfrak{X}$ are $\mathbb{S}$-conjugate only if they are $N(\mathfrak{X})$-conjugate.

Let $\mathfrak{X}=\mathfrak{X}_{1} \times \mathfrak{X}_{2} \times \mathfrak{X}_{3}$, where $\left|\mathfrak{X}_{i}\right|=3$ and where $\mathfrak{X}_{i}$ admits $\mathfrak{B}, i=$ 1, 2,3. Let $\mathfrak{B}_{i}=C\left(\mathfrak{X}_{i}\right) \cap \mathfrak{B}=\left\langle V_{i}\right\rangle$. Thus, $\mathfrak{X}_{1}, \mathfrak{X}_{2}, \mathfrak{X}_{3}$ are the only subgroups of $\mathfrak{X}$ of order 3 which admit $\mathfrak{B}$. Let $Z$ be a generator for 3. Then $Z=X_{1} X_{2} X_{3}$ with $X_{i} \in \mathfrak{X}_{i}$.

We argue that $3 \nsim \mathfrak{X}_{i}$ for $i=1,2,3$. Namely, if $3 \sim \mathfrak{X}_{i}$, there is $N \in N(\mathfrak{X})$ such that $\mathfrak{X}_{i}=\mathfrak{3}^{N}$. Let $\mathfrak{A}=\boldsymbol{A}_{\mathscr{S}}(\mathfrak{X})$. Thus, $|\mathfrak{A}|_{3}=3, \mathfrak{A}$ is solvable, and $\mathfrak{A} \supseteqq \mathfrak{B}=\boldsymbol{A}_{\mathfrak{Q}}(\mathfrak{X}) \cong \Sigma_{4}$. So $\mathfrak{A}=\mathfrak{B}$ or $\mathfrak{A}=\mathfrak{B} \times \mathfrak{A}_{0}$, where $\mathfrak{A}_{0}=\langle A\rangle$ and $A$ inverts $\mathfrak{X}$. In neither case are 3 and $\mathfrak{X}_{i}$ in the same orbit under $\mathfrak{A}$.

We now return to our study of $\mathfrak{S}$. Let $\mathfrak{S}_{2}=\mathfrak{Q} \times\langle V\rangle$, where $\mathfrak{Q}$ is a quaternion group and $V \in \mathfrak{B}$. Let $\left\langle V_{0}\right\rangle=\mathfrak{Q}$ '. Since $V_{0}$ does not centralize $\boldsymbol{Z}\left(\boldsymbol{O}_{3}(\mathfrak{S})\right)$, there is a minimal normal subgroup $\mathfrak{Y}$ of $\mathfrak{S}$ such that $\mathfrak{Q}$ is represented faithfully on $\mathfrak{Y}$. Let $\mathfrak{B}^{*}=\boldsymbol{C}_{\mathfrak{B}}(\mathfrak{Y})$ so that $\left|\mathfrak{B}^{*}\right|=$ $2, \mathfrak{S}_{2}=\mathfrak{Q} \times \mathfrak{B}^{*}$. We see that $|\mathfrak{Y}|=9$ and that $\mathfrak{Y}_{0}=\mathfrak{Y} \cap \boldsymbol{Z}\left(\mathfrak{S}_{3}\right)$ is of order 3 and admits $\mathfrak{B}$. Thus, $\mathfrak{Y}_{0} \subset \mathfrak{X}$, so $\mathfrak{Y}_{0}=\mathfrak{X}_{i}$ for some $i=1,2,3$. Since $\mathfrak{X}_{i} \nsim 3$, it follows that $\mathfrak{S}_{3}$ is a $S_{3}$-subgroup of $C\left(\mathfrak{Y}_{0}\right)$. Since $\left[\mathfrak{Y}, \mathfrak{S}_{3}\right] \subseteq \mathfrak{Y}_{0}$, it follows that $\mathfrak{Y} \subseteq \boldsymbol{O}_{3}\left(\boldsymbol{C}\left(\mathfrak{V}_{0}\right)\right)$. Since $\mathfrak{Q}$ permutes transitively the subgroups of $\mathfrak{Y}$ of order 3 , it follows that $\mathfrak{Y} \subseteq \boldsymbol{O}_{3}\left(\boldsymbol{C}(\mathfrak{Y})^{*}\right)$ ) for every subgroup $\mathfrak{Y} *$ of $\mathfrak{Y}$ of order 3 . This implies that $\mathfrak{Y} \in \mathscr{E}(3)$. Now $\boldsymbol{C}\left(\mathfrak{B}^{*}\right)$ contains $\mathfrak{Y}$ and also contains an element of $\mathscr{U}(2)$, so $C\left(\mathfrak{B}^{*}\right)$ is nonsolvable. This contradiction shows that this case does not arise.

\section{Case 2. $\boldsymbol{O}_{3}(\mathfrak{S}) \mathfrak{B} / \boldsymbol{O}_{3}(\mathfrak{S}) \nsubseteq Z\left(\mathfrak{S} / \boldsymbol{O}_{3}(\Im)\right)$.}

We conclude that $\mathfrak{S}_{2}$ is special and that $\mathfrak{B}=\mathfrak{S}_{2}^{\prime}$. Since $\mathfrak{B}=Z\left(\mathfrak{S}_{2}\right)$, we get that $\mathfrak{S}_{3} \mathfrak{B}$ is a maximal subgroup of $\mathfrak{S}$. That is, $\boldsymbol{O}_{3}(\mathfrak{S}) \mathfrak{S}_{2} / \boldsymbol{O}_{3}(\mathfrak{S}) \mathfrak{B}$ is a chief factor of $\mathfrak{S}$.

Let $\mathfrak{P}_{0}$ be a maximal element of $\boldsymbol{U}_{\widetilde{\Xi}}(\mathfrak{B} ; 3)$ with $\mathfrak{P}_{0} \subset \mathfrak{S}_{3}$. Hence, $\mathfrak{P}_{0}$ is of index 3 in $\mathfrak{S}_{3}$, and all involutions of $\mathfrak{B}$ are fused in $\mathfrak{S}_{3} \mathfrak{B}$. Also, $\left[\mathfrak{B}_{0}, \mathfrak{P}_{0}\right]=\left[\boldsymbol{O}_{3}(\mathfrak{S}), \mathfrak{B}_{0}\right]$ for every subgroup $\mathfrak{B}_{0}$ of $\mathfrak{B}$.

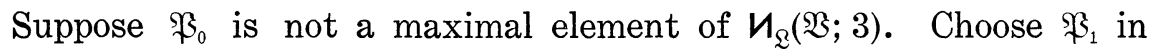
$\boldsymbol{U}_{\mathfrak{Q}}(\mathfrak{B} ; 3)$ so that $\left|\mathfrak{P}_{1}: \mathfrak{P}_{0}\right|=3$, and let $\mathfrak{B}_{0}=\boldsymbol{C}_{\mathfrak{B}}\left(\mathfrak{P}_{1} / \mathfrak{P}_{0}\right)$. Then $\mathfrak{P}_{1}$ and $\mathfrak{S}_{2}$ both normalize $\left[\mathfrak{B}_{0}, \mathfrak{P}_{1}\right]$. Let $\mathfrak{2}^{*}$ be a $S_{2,3}$-subgroup of $N\left(\left[\mathfrak{B}_{0}, \mathfrak{P}_{1}\right]\right)$ which contains $\mathfrak{B}_{1}$, and let $\mathfrak{R}_{p}^{*}$ be a $S_{p}$-subgroup of $\mathfrak{R}^{*}$ with $\mathfrak{P}_{1} \subseteq \Omega_{3}^{*}$, $\mathfrak{B} \subseteq \mathfrak{R}_{2}^{*}$. Note that $\mathfrak{Q}^{*}$ contains a conjugate of $\mathfrak{S}_{2}$.

By maximality, $\mathfrak{S}_{3}=N_{\mathfrak{\beta}}\left(\Omega_{1}\left(Z\left(O_{3}(\Im)\right)\right)\right)$. Hence, $3 \subset \widetilde{S}_{3}$, and so $3 \subseteq$ $\Omega_{1}\left(Z\left(O_{3}(\mathfrak{S})\right)\right)$, since $\boldsymbol{O}_{2}(\mathfrak{S})=1$. If $\mathfrak{U} \in \mathscr{U}(\mathfrak{P})$, then $[\mathfrak{P}, \mathfrak{M}] \subseteq \mathcal{Z}$, and so 
$\mathfrak{u} \subseteq \mathfrak{S}_{3}$. Since $\mathfrak{B}$ is a 4-group and does not centralize $\Omega_{1}\left(Z\left(\boldsymbol{O}_{3}(\mathfrak{S})\right)\right)$, we conclude from $[\mathfrak{P}, \mathfrak{u}, \mathfrak{u}]=1$ that $\mathfrak{u}$ centralizes $\mathfrak{B} \boldsymbol{O}_{3}(\mathfrak{S}) / \boldsymbol{O}_{3}(\mathfrak{S})$. That is, $\mathfrak{U} \subseteq \mathfrak{P}_{0} \subseteq \mathbb{2}^{*}$. Hence, $\mathbb{2}^{*}$ contains an element of $\mathscr{S}$ so maximality of $\mathfrak{S}$ guarantees that $\mathfrak{P}_{1}=\mathfrak{R}_{3}^{*}$, since $\mathfrak{P}_{1}$ and $\mathfrak{S}_{3}$ are of the same order.

Let $\Omega_{2}^{* *}=\Omega_{2}^{*} \cap \boldsymbol{O}_{3,2}\left(\Omega^{*}\right)$. Thus, $\Omega_{2}^{* *}$ contains a noncyclic abelian subgroup of order 8 , since $\Omega^{*}$ contains a conjugate of $\mathfrak{S}_{2}$.

Suppose every subgroup of $\mathfrak{\Omega}_{2}^{* *}$ which is characteristic and abelian is also cyclic. Let $\mathbb{R}_{2}^{* * *}$ be a subgroup of $\mathbb{R}_{2}^{* *}$ which is minimal subject to (a) containing a noncyclic abelian subgroup of order 8 and (b) being permutable with $\mathfrak{\Omega}_{3}^{*}$. Then since $\boldsymbol{D}\left(\Omega_{2}^{* * *}\right) \subseteq \boldsymbol{D}\left(\mathbb{R}_{2}^{* *}\right)$, it follows that $\mathfrak{\Omega}_{2}^{* * *}$ is not a special group with center of order 4 . Since $\mathfrak{R}_{3}^{*} \mathfrak{R}_{2}^{* * *} \in \mathscr{S}$, our previous reduction excludes this possibility.

Let $\tilde{\Omega}_{2}$ be a noncyclic characteristic abelian subgroup of $\Omega_{2}^{* *}$. If $\left|\tilde{\mathfrak{L}}_{2}\right|>4$, then $\mathfrak{F}_{1} \tilde{\mathfrak{R}}_{2}$ contains an element of $\mathscr{S}$, against our previous reduction. We may assume that $\tilde{\Omega}_{2}$ is a four-group. If $\tilde{\Omega}_{2} \cap \mathfrak{B} \neq 1$, then $\boldsymbol{O}_{3}\left(\mathbb{2}^{*}\right) \tilde{\mathbb{R}}_{2} / \boldsymbol{O}_{3}\left(\mathbb{2}^{*}\right)$ is centralized by $\mathfrak{P}_{1}$, since $\mathfrak{B}$ normalizes $\mathfrak{P}_{1}$. But in this case, there are maximal elements of $\mathscr{S}$ which do not satisfy our previous reduction. If $\tilde{\mathfrak{L}}_{2} \cap \mathfrak{B}=1$, then $\mathfrak{P}_{1} \mathfrak{B} \tilde{\mathfrak{R}}_{2}$ contains an element of $\subseteq$ which also violates our previous reduction. Hence, $\mathfrak{P}_{0}$ is a maximal element of $\boldsymbol{U}_{\mathfrak{L}}(\mathfrak{B} ; 3)$.

Since $O_{3}(\mathfrak{Q}) \in N_{\mathfrak{Q}}(\mathfrak{B} ; 3)$, we have $\boldsymbol{O}_{3}(\mathfrak{Q}) \subseteq \mathfrak{P}_{0}$. Maximality of $\mathfrak{S}$ in $\mathscr{S}$ implies that $\mathbb{R}$ contains no noncyclic abelian subgroup of order 8 . Since the involutions of $\mathfrak{B}$ are fused in $\mathfrak{S}_{3} \mathfrak{B}$, it follows that $\boldsymbol{O}_{3,2}(\mathbb{R})=\boldsymbol{O}_{3}(\mathbb{R}) \mathfrak{B}$. Hence, $\mathfrak{P}_{0}=O_{3}(\mathbb{R})$ is of index 3 in $\mathbb{R}_{3}$. This violates $\left|\mathfrak{R}_{3}\right|>\left|\mathfrak{S}_{3}\right|=3\left|\mathfrak{P}_{0}\right|$.

Thus, in all cases, we have shown that $\mathfrak{S}_{3}=\mathfrak{P}$.

Suppose that $\mathfrak{B}$ normalizes $\mathfrak{S}_{3}$. Let $\mathfrak{W}$ be a minimal normal subgroup of $\mathfrak{S}$. Clearly, $\mathfrak{W} \supset 3$. Since $\mathfrak{B} \boldsymbol{O}_{3}(\mathfrak{S}) / \boldsymbol{O}_{3}(\mathfrak{S})$ is a central factor of $\mathfrak{S}$, some involution of $\mathfrak{B}$ centralizes $\mathfrak{W}$. But $\mathfrak{W}$ contains an element of $\mathscr{U}(\mathfrak{P})$, so Lemmas 7.4 and 5.38 imply that $C(V)$ is nonsolvable for some involution $V$ of $\mathfrak{B}$. Thus, $\mathfrak{B}$ does not normalize $\mathfrak{P}$. In particular, $\Im_{2}$ is special.

Let $\mathfrak{P}_{0}$ be the largest subgroup of $\mathfrak{P}$ normalized by $\mathfrak{B}$. Thus, $\left|\mathfrak{P}: \mathfrak{P}_{0}\right|=3$ and $\boldsymbol{N}_{\mathfrak{P}}\left(\mathfrak{S}_{2}\right)$ permutes transitively the involutions of $\mathfrak{B}$.

Let $\mathfrak{W}$ be a minimal normal subgroup of $\mathfrak{S}$. Clearly, $\mathfrak{W} \supset 3$, so $\mathfrak{B}$ is faithfully represented on $\mathfrak{W}$. Hence, $\mathfrak{S}_{2}$ is faithfully represented on $\mathfrak{W}$, so $\boldsymbol{C}_{\mathfrak{S}}(\mathfrak{W})=\boldsymbol{O}_{3}(\mathfrak{W})$. Let $\mathfrak{W}=\mathfrak{W}_{1} \times \mathfrak{W}_{2} \times \mathfrak{W}_{3}$, where $\mathfrak{W}_{i}=\boldsymbol{C}_{\mathfrak{W}}\left(\mathfrak{B}_{i}\right)$ and $V_{1}, V_{2}, V_{3}$ are the involutions of $\mathfrak{B}$. Thus, $\mathfrak{S}_{2} \mathfrak{P}_{0}$ normalizes each $\mathfrak{W}_{i}$, and $\mathfrak{S}$ permutes $\mathfrak{W}_{1}, \mathfrak{W}_{2}, \mathfrak{W}_{3}$ transitively. Obviously each $\mathfrak{W}_{i}$ is an irreducible $\mathfrak{B}_{0} \mathfrak{\subseteq}_{2}$-module.

Let $\Re_{i}=C_{\mathfrak{\Re}_{0} \mathfrak{\subseteq}_{2}}\left(\mathfrak{W}_{i}\right)$, and $\mathfrak{S}_{2 i}=\left\{S \in \mathfrak{S}_{2} \mid\left[S, \mathfrak{S}_{2}\right] \subseteq\left\langle V_{i}\right\rangle\right\}$, for $i=1,2,3$. Then $\boldsymbol{N}_{\mathfrak{S}}\left(\mathfrak{S}_{2}\right)$ permutes $\left\{\mathfrak{S}_{21}, \mathfrak{S}_{22}, \mathfrak{S}_{23}\right\}$ transitively, and so one of the following holds:

(a) $\mathfrak{S}_{2 i}=\mathfrak{B}, i=1,2,3$,

(b) $\quad \mathfrak{S}_{2 i} \supset \mathfrak{B}, i=1,2,3$. 
If (a) holds, then $\mathfrak{S}_{2} /\left\langle V_{i}\right\rangle$ is extra special, and since $V_{j}$ inverts $\mathfrak{B}_{i}$ for $j \neq i$, it follows that $\Re_{i} \cap \mathfrak{S}_{2}=\left\langle V_{i}\right\rangle$, whence $\Re_{i}=\boldsymbol{O}_{3}(\mathfrak{S})\left\langle V_{i}\right\rangle$. The proof of Lemma 5.52 now shows that $\boldsymbol{O}_{3}(\mathfrak{S}) \supseteqq \boldsymbol{J}\left(\mathfrak{S}_{3}\right)$, the desired contradiction.

Suppose (b) holds. The group $\boldsymbol{O}_{3}\left(\mathfrak{S}_{)} \mathfrak{S}_{21} \mathfrak{S}_{22} \mathfrak{S}_{23}\right.$ is clearly $\mathfrak{S}_{3}$-invariant, and since $\boldsymbol{O}_{3}(\mathfrak{S}) \mathfrak{S}_{2} / \boldsymbol{O}_{3}(\mathfrak{S}) \mathfrak{B}$ is a chief factor of $\mathfrak{S}$, we have $\mathfrak{S}_{2}=\mathfrak{S}_{21} \mathfrak{S}_{22} \mathfrak{S}_{23}$. Obviously, $\left[\mathfrak{S}_{2 i}, \mathfrak{S}_{2 j}\right] \subseteq\left\langle V_{i}\right\rangle \cap\left\langle V_{j}\right\rangle=1$ for $i \neq j$. Because $\mathfrak{S}_{2}$ is special, we conclude that $\mathfrak{S}_{2 i^{\prime}}=\left\langle V_{i}\right\rangle$ and hence that $\mathfrak{S}_{2 j} \mid\left\langle V_{i}\right\rangle$ is extra special for $i \neq j$. If the width of $\Im_{2_{j}} \mid\left\langle V_{i}\right\rangle$ is greater than 1 , then since $\boldsymbol{J}\left(\mathfrak{S}_{3}\right) \nsubseteq \boldsymbol{O}_{3}\left(\mathfrak{S}_{)}\right.$, and since $\mathfrak{S}_{2 j} /\left\langle V_{i}\right\rangle$ acts faithfully on $\mathfrak{W}_{i}$, it follows that $\boldsymbol{J}\left(\mathfrak{S}_{3}\right)$ centralizes $\boldsymbol{O}_{3}\left(\mathfrak{S}_{)} \mathfrak{S}_{2 j} / \boldsymbol{O}_{3}(\mathfrak{S})\left\langle V_{i}\right\rangle\right.$. But $\boldsymbol{J}\left(\mathfrak{S}_{3}\right) \triangleleft \mathfrak{S}_{3}$, and so $\boldsymbol{J}\left(\mathfrak{S}_{3}\right)$ centralizes $\boldsymbol{O}_{3}(S) \mathfrak{S}_{2} / \boldsymbol{O}_{3}\left(\Im_{)}\right) \mathfrak{B}$, that is, $\boldsymbol{J}\left(\mathfrak{S}_{3}\right) \subseteq \boldsymbol{O}_{3}\left(\mathfrak{S}_{)}\right.$. We may assume that if $\mathrm{i} \neq j$, then $\mathfrak{S}_{2 i} \mid\left\langle V_{i}\right\rangle$ is of width 1 . But then $\mathfrak{S}_{21} \mathfrak{S}_{22} /\left\langle V_{3}\right\rangle$ is the central product of $\mathfrak{S}_{21} \mid\left\langle V_{3}\right\rangle$ and $\mathfrak{S}_{22} /\left\langle V_{3}\right\rangle$, so is extra special of width 2, acts faithfully on $\mathfrak{W}_{3}$, and $\boldsymbol{O}_{3}\left(\mathfrak{S}_{)} \mathfrak{S}_{21} \mathfrak{S}_{22}\right.$ admits $\boldsymbol{J}\left(\mathfrak{S}_{3}\right)$. By Lemma 5.52, $\boldsymbol{J}\left(\Im_{3}\right)$ centralizes $\boldsymbol{O}_{3}\left(\Im_{)} \Im_{21} \Im_{22} / \boldsymbol{O}_{3}(\Im)\right.$, so again we get the contradiction $\boldsymbol{J}\left(\Im_{3}\right) \subseteq \boldsymbol{O}_{3}\left(\Im_{S}\right)$. The proof is complete.

LEMMA 9.16. If $\mathfrak{A}$ is a subgroup of $(3)$ of type $(3,3)$ and each element of $\mathfrak{X}$ centralizes an element of $\mathscr{Q}(3)$, then

(i ) $\mathfrak{A} \in \mathscr{D}$.

(ii) $4 \nmid|\boldsymbol{C}(\mathfrak{H})|$.

Proof. (i) Suppose false, and $\mathfrak{I}$ is a four-group normalized but not centralized by $\mathfrak{A}$. Let $\mathfrak{U}_{0}=C_{\mathfrak{U}}(\mathfrak{T})$, so that $\left|\mathfrak{U}_{0}\right|=3$. Let $\mathfrak{\Omega}_{0}$ be a maximal 2, 3-subgroup of (S) which contains a $S_{2,3}$-subgroup of $C\left(\mathfrak{H}_{0}\right)$ containing $\mathfrak{Y T}$. Let $\mathbb{L}=\boldsymbol{O}^{3^{\prime}}\left(\mathbb{L}_{0}\right)$. Since $\mathbb{R}$ contains an element of $\mathscr{E}(3)$, Lemma 9.15 implies that $\&$ contains no noncyclic abelian subgroup of order 8. Hence, $\mathfrak{I}$ is a $S_{2}$-subgroup of $\mathbb{Z}$ and $\mathbb{L} / O_{3}(\mathbb{R}) \cong A_{4}$. Let $\mathbb{R}_{3}$ be a $S_{3}$-subgroup of $\mathbb{2}$. Since $\mathfrak{I}$ does not centralize $\boldsymbol{Z}\left(\boldsymbol{O}_{3}(\mathbb{R})\right)$, it follows that $\boldsymbol{J}\left(\mathbb{R}_{3}\right) \triangleleft \mathbb{R}$. Hence, $\mathbb{R}_{3}$ is a $S_{3}$-subgroup of $\mathbb{S}$, and we may assume that $\mathbb{\Omega}_{3}=\mathfrak{P}$.

Let $\mathfrak{X}$ be a minimal normal subgroup of $\mathbb{Q}$ with $\mathfrak{X} \subseteq Z(J(\mathfrak{P}))$. Thus, $\mathfrak{X}$ is elementary of order 27 and $C_{\mathfrak{X}}(\mathfrak{I})=1$. Choose $T$ in $\mathfrak{I}^{*}$ so that $\mathfrak{X}_{1}=C_{\mathfrak{X}}(T)$ is of order 3 and is inverted by the generator of $\mathfrak{T} /\langle T\rangle$. Hence, $\left\langle\mathfrak{X}_{0}, \mathfrak{X}_{1}\right\rangle=\mathfrak{U}^{*}$ is elementary of order 9 and is normalized by $\mathfrak{T}$, and every element of $\mathfrak{U}^{*}$ centralizes an element of $\mathscr{U}(\mathfrak{F})$.

Let $\mathfrak{C}$ be a $S_{2,3}$-subgroup of $\boldsymbol{C}(T)$ which contains $\mathfrak{2} * \mathfrak{T}$. By Lemma 5.38, $\sqrt{5}$ contains an element of $\mathscr{U}(2)$, so $\left|\boldsymbol{O}_{3}(\mathfrak{S})\right| \leqq 3$, and $\boldsymbol{O}_{3}(\mathfrak{S}) \cap \mathfrak{A}^{*}=$ 1. Hence, $\mathfrak{A}^{*}$ is faithfully represented on $\boldsymbol{O}_{2}(\mathfrak{E})$. Let $\mathfrak{S}_{0}$ be a characteristic abelian subgroup of $\boldsymbol{O}_{2}(\mathfrak{C})$. Suppose $\mathfrak{U} *$ does not centralize $\mathfrak{C}_{0}$. Hence, there is an element $A$ in $\mathfrak{2}^{* *}$ such that $C(A)$ contains an elementary subgroup of order 8 . This is not the case, so $\mathfrak{A}^{*}$ centralizes $\mathfrak{S}_{0}$. If $\left|\mathfrak{S}_{0}\right|>2$, then some element $A$ of $\mathfrak{U}^{* *}$ centralizes a noncyclic 
abelian subgroup of $\boldsymbol{O}_{2}(\mathbb{E})$ of order 8. This is not the case, by Lemma 9.15. Hence, $O_{2}(\mathbb{S})$ is extra special of width at least 2 and $\langle T\rangle=O_{2}(\mathfrak{S})^{\prime}$. Hence, (5) contains a $S_{2}$-subgroup of (s).

By Lemma 9.15, no element of $\mathfrak{U}^{* \sharp}$ centralizes any noncyclic abelian subgroup of order 8. Hence, $\langle T\rangle=\boldsymbol{O}_{2}(\mathfrak{E}) \cap \boldsymbol{C}\left(\mathfrak{H}^{*}\right)$. For each $A$ in $\mathfrak{X}^{* *}$, $\boldsymbol{O}_{2}(\mathbb{S}) \cap \boldsymbol{C}(A)$ is either $\langle T\rangle$ or is extra special. Thus, $\boldsymbol{O}_{2}(\mathbb{S}) \cap \boldsymbol{C}(A)$ is either $\langle T\rangle$ or is a quaternion group, so no element of $\mathfrak{A}^{* \sharp}$ centralizes any four-subgroup of $\boldsymbol{O}_{2}(\mathbb{E})$. Thus, the width of $\boldsymbol{O}_{2}(\mathbb{E})$ is at most 4 . Since $\boldsymbol{O}_{2}(\mathfrak{S}) \cap \boldsymbol{C}\left(\mathfrak{U}_{0}\right)$ is centralized by $\mathfrak{U}^{*}$, it follows that $\boldsymbol{O}_{2}(\mathfrak{S}) \cap \boldsymbol{C}\left(\mathfrak{U}_{0}\right)=$ $\langle T\rangle$, and so the width of $\boldsymbol{O}_{2}(\mathfrak{S})$ is at most 3 .

Consider $C^{*}\left(\mathfrak{X}_{1}\right)=\left\{G \in \mathbb{S}, G\right.$ either centralizes or inverts $\left.\mathfrak{X}_{1}\right\}$. By construction, $\left|\boldsymbol{C}_{\mathbb{Q}}\left(\mathfrak{X}_{1}\right)\right|_{3}=|\mathfrak{F}| / 3$. Also, $\mathfrak{I} \subseteq C^{*}\left(\mathfrak{X}_{1}\right)$, and $C^{*}\left(\mathfrak{X}_{1}\right)$ contains no noncyclic abelian subgroup of order 8. Suppose $\mathfrak{U}_{0} \nsubseteq \boldsymbol{O}_{3}(\tilde{\Omega})$, where $\tilde{\mathfrak{L}}$ is a $S_{2,3}$-subgroup of $C^{*}\left(\mathfrak{X}_{1}\right)$ which contains $\boldsymbol{C}_{\mathfrak{\Omega}}^{*}\left(\mathfrak{X}_{1}\right)$. Then $\tilde{\mathfrak{\Omega}} / \boldsymbol{O}_{3}(\tilde{\mathfrak{L}})$ contains a subgroup isomorphic to $\mathfrak{T}_{0} \times \mathfrak{I}$. This is obviously impossible, since $S_{2}$-subgroups of $\tilde{\mathfrak{L}} / \boldsymbol{O}_{3}(\tilde{\mathfrak{L}})$ are of maximal class. Hence, $\mathfrak{N}_{0} \leqq \boldsymbol{O}_{3}(\tilde{\mathfrak{L}})$. This implies that $\mathfrak{U}_{0}$ centralizes $\boldsymbol{O}_{2}(\mathfrak{E}) \cap C\left(\mathfrak{X}_{1}\right)$, so the width of $\boldsymbol{O}_{2}(\mathfrak{E})$ is 2. Hence, $\mathfrak{X}_{1} \times \mathfrak{A}_{0}$ is a $S_{3}$-subgroup of $C(T)$, so $\mathfrak{X}_{1} \times \mathfrak{N}_{0}$ is a $S_{3}$ subgroup of $C_{\Omega}(T)$. By a formula of Wielandt [40],

$$
\left|\boldsymbol{O}_{3}(\mathbb{Q})\right|=\left|\boldsymbol{O}_{3}(\mathbb{2}) \cap \boldsymbol{C}(T)\right|^{3} /\left|\boldsymbol{O}_{3}(\mathbb{Q}) \cap \boldsymbol{C}(\mathfrak{T})\right|^{2} .
$$

Hence, $\left|\boldsymbol{O}_{3}(\mathbb{R})\right|=3^{6} / 3^{2}=3^{4}$, so that $\boldsymbol{O}_{3}(L)=\mathfrak{U}_{0} \times \mathfrak{X}$. This implies that $\boldsymbol{Z}(\mathfrak{P})$ is noncyclic, since $\left|\mathfrak{P}: \boldsymbol{O}_{3}(\mathbb{2})\right|=3$. The proof of (i) is complete.

As for (ii), suppose $\mathfrak{I}$ is a subgroup of $C(\mathfrak{I})$ of order 4 . Then $C(T)$ contains an element of $\mathscr{C}(2), T$ being an involution of $\mathfrak{T}$. Thus, by Lemma 7.5, there is a subgroup $\mathfrak{M}$ in $\mathscr{C S}$ (S) which contains $\mathfrak{V} \mathfrak{I}$ and satisfies $\boldsymbol{O}_{2^{\prime}}(\mathfrak{M})=1$, while $\boldsymbol{O}_{2}(\mathfrak{M})$ is of symplectic type. Since $\mathfrak{A}$ acts faithfully on $\boldsymbol{O}_{2}(\mathfrak{M}) \cap \boldsymbol{C}(\mathfrak{I})$, we can therefore choose $A$ in $\mathfrak{U}^{\sharp}$ such that $\mathfrak{A}$ does not centralize $\boldsymbol{O}_{2}(\mathfrak{C}) \cap \boldsymbol{C}(\mathfrak{I}) \cap \boldsymbol{C}(\mathfrak{H})$. Thus, $\boldsymbol{C}(A)$ contains a noncyclic abelian subgroup of order 8, against Lemma 9.15. The proof of (ii) is complete.

Lemma 9.17. Suppose

(a) $\Re$ is a maximal 2, 3-subgroup of (5).

(b) $\Re$ contains an element of $\mathscr{D}$.

(c) $\Re$ contains a noncyclic abelian subgroup of order 8. Then $\boldsymbol{O}_{2}(\Re) \neq 1$.

Proof. Let $\Re_{p}$ be a $S_{p}$-subgroup of $\Re, p=2$, 3. We assume without loss of generality that $\Re_{3} \subseteq \mathfrak{P}$. Suppose by way of contradiction that $\boldsymbol{O}_{2}(\Re)=1$. Then $\boldsymbol{O}_{3}(\Re) \neq 1$, so by maximality of $\Re, \Re_{3}=\boldsymbol{N}_{\mathfrak{\beta}}\left(\boldsymbol{O}_{3}(\Re)\right)$. Hence, $3 \subseteq \Re_{3}$. Since $\boldsymbol{O}_{2}(\Re)=1$, we get $3 \subseteq Z\left(O_{3}(\Re)\right)$. Hence, $\Re_{3}$ contains every element of $\mathscr{C}(\mathfrak{P})$. This contradicts Lemma 9.15 and completes the proof. 
We now begin the construction of the final configuration.

By hypothesis, $2 \sim 3$. Let $\mathfrak{A}$ be a noncyclic abelian subgroup of (S) of order 8 and let $\mathfrak{B}$ be an elementary subgroup of order 9 each of whose elements centralizes an element of $\mathscr{U}(3)$, chosen so that $\langle\mathfrak{X}, \mathfrak{B}\rangle$ is solvable. We assume without loss of generality that $\langle\mathfrak{U}, \mathfrak{B}\rangle$ is a 2,3-group. Let $\mathfrak{\&}$ be a maximal 2,3-subgroup of (s) which contains $\langle\mathfrak{A}, \mathfrak{B}\rangle$.

Let $\mathfrak{R}_{p}$ be a $S_{p}$-subgroup of $\mathfrak{R}, p=2,3$, with $\mathfrak{B} \subseteq \mathfrak{R}_{3}$. By Lemma 9.17, $O_{2}(\Omega) \neq 1$.

Let $J$ be an involution in $\boldsymbol{Z}\left(\mathfrak{Q}_{2}\right) \cap \boldsymbol{O}_{2}(\mathfrak{R})$. Since $\mathfrak{B} \in \mathscr{D}$, by Lemma 9.16, $\mathfrak{B}$ centralizes $Z\left(O_{2}(\mathfrak{Q})\right)$. Hence, $C(J)$ is a solvable subgroup containing $\mathfrak{B}, \mathfrak{Q}_{2}$, and an element of $\mathscr{U}(2)$.

Since $\mathfrak{B} \in \mathscr{D}$, we may apply Lemma 7.5. Let $\mathfrak{M}$ be an element of $\mathscr{A S}(\mathbb{S})$ which contains $\mathfrak{B}$ and $\mathbb{S}_{2}$ and which satisfies all the conclusions of Lemma 7.5. Let $\Re$ be a $S_{2,3}$-subgroup of $\mathfrak{M}$ and let $\Re_{0}=O_{2}(\Re)$. Since $\boldsymbol{O}_{2^{\prime}}(\mathfrak{M})=1$, so also $\boldsymbol{O}_{3}(\Re)=1$. Since no element of $\mathfrak{B}^{\sharp}$ centralizes any noncyclic abelian subgroup of order 8 , it follows that $\Re_{0}$ is extra special of width 2,3 or 4 , and $C_{\Re_{0}}(\mathfrak{B})=\Re_{0}^{\prime}=\langle I\rangle$, the last equality serving to define $I$. Hence, $\Re_{0}=\boldsymbol{O}_{2}(\mathfrak{M})$. Let $\Re_{p}$ be a $S_{p}$-subgroup of $\Re, p=2$, 3, with $\mathfrak{B} \cong \Omega_{3}$. Let $\Re_{3}^{*}=\Re_{3} \cap \boldsymbol{O}_{2,3}(\Re), \AA^{*}=N_{\Re}\left(\Re_{3}^{*}\right)$. Thus, $\Re=\Re_{0} \Omega^{*}$ and $\Re_{0} \cap \Re^{*}=C_{\Re_{0}}\left(\AA_{3}^{*}\right)$. Let $\Re_{2}^{*}=\Re^{*} \cap \Re_{2}$ so that $\Re^{*}=\Re_{3} \Re_{2}^{*}$. We assume without loss of generality that $\Re_{3} \subseteq \mathfrak{P}$.

We argue that

$$
\mathfrak{R}_{0} \cap \Re^{*}=\langle I\rangle .
$$

Namely, choose $\mathfrak{U}$ in $\mathscr{C}(\mathfrak{P})$ and suppose $C(\mathfrak{U}) \cap \Re_{3}^{*}$ is noncyclic. Then by Lemma 9.16 (ii), no noncyclic abelian subgroup of $C(\mathfrak{U}) \cap \Re_{3}^{*}$ centralizes any subgroup of order 4 , so (9.25) is clear. Suppose $\boldsymbol{C}(\mathfrak{U}) \cap \Re_{3}^{*}$ is cyclic. Hence, $\Re_{3}^{*}$ has a cyclic subgroup of index 3 . Assume that $(9.25)$ does not hold. Then $\mathfrak{B} \nsubseteq \Re_{3}^{*}$, so the 3 -length of $\Re$ is at least 2 . Hence, $\Omega_{3}^{*}$ is elementary of order 9 and all elements of $\Re_{3}^{* *}$ are fused in $\Re$. But then every element of $\Re_{3}^{*}$ centralizes an element of $\mathscr{U}(3)$, so again (9.25) holds. Thus, (9.25) holds.

Lemma 9.18. If $\Re$ is any 2, 3-subgroup of (S) which contains $\mathfrak{B}, I$, and also contains a noncyclic abelian subgroup of order 8 , then $\mathfrak{R} \subseteq \mathfrak{M}$.

Proof. We may assume that $\Re$ is a maximal 2, 3-subgroup of $(3)$. By Lemma 9.17, we have $\boldsymbol{O}_{2}(\Re) \neq 1$. Since $\mathfrak{B} \in \mathscr{D}, \mathfrak{B}$ centralizes $\boldsymbol{Z}\left(\boldsymbol{O}_{2}(\Re)\right)$. Since $\langle I\rangle$ is a $S_{2}$-subgroup of $C(\mathfrak{B})$, by Lemma 9.16 (ii), it follows that $\langle I\rangle=Z\left(O_{2}(\Re)\right)$, so $\Re \cong C(I)=\mathfrak{M}$.

LEMMA 9.19. $\Re_{2}^{*}$ contains no noncyclic abelian subgroup of order 8. 
Proof. Suppose false. In this case, $\Re^{*}$ is a $S_{2,3}$-subgroup of $N\left(\Re_{3}^{*}\right)$, by the preceding paragraph. Hence, the 3-length of $\Omega^{*}$ is at least 2 . But in this case, $3 \subset \Re_{3}^{*}$, so $\Re_{3}$ contains every element of $\mathscr{C}(\mathfrak{P})$, against Lemma 7.4. The proof is complete.

Lemma 9.20. If $\widetilde{\Re}_{3}$ is a $S_{3}$-subgroup of $N\left(\Re_{3}\right)$, then

$$
\tilde{\Re}_{3}=\Re_{3} \cdot C_{\widetilde{\Re}_{3}}\left(\Re_{3}\right) \text {. }
$$

Proof. Let $\mathfrak{夭}_{1}=C\left(\Re_{3}\right) \Re_{3}, \Re_{1}=N\left(\Re_{3}\right)$. Since $\mathfrak{B} \subseteq \Re_{3}$, it follows that $\langle I\rangle$ is a $S_{2}$-subgroup of $\mathfrak{F}_{1}$, by Lemma 9.16 (ii). Hence, $\mathfrak{M}$ covers $\mathfrak{R}_{1} / \mathfrak{S}_{1}$, so $\mathfrak{E}_{1}$ contains $\mathfrak{R}_{3}$, which is equivalent to our assertion.

LEMMA 9.21.

(a) If $\tilde{\mathfrak{P}}$ is any 3-subgroup of $\mathfrak{M}$, then no $S_{3}$-subgroup of $N(\tilde{\mathfrak{P}})$ is contained in any conjugate of $\mathfrak{M}$.

(b) If $P$ is an element of $\mathfrak{M}$ of order 3 , then $C(P)$ contains a subgroup $\mathfrak{X}^{*}$ of type $(3,3)$ such that $C(A)$ contains an element of $\mathscr{Q}(3)$ for each $A$ in $\mathfrak{U}^{*}$.

( c) If $\tilde{\mathfrak{B}}$ is any nonidentity 3-subgroup of $\mathfrak{M}$, then $N(\tilde{\mathfrak{R}})$ contains no noncyclic abelian group of order 8.

(d) $\Omega_{3}$ contains no abelian subgroup of order 27 .

( e ) $\Re_{3}$ is isomorphic to one of the following groups:

(i) an elementary group of order 9.

(ii) a nonabelian group of order 27.

Proof. Let $\mathfrak{P}^{*}$ be a $S_{3}$-subgroup of $N(\tilde{\mathfrak{R}})$. Suppose $\mathfrak{P}^{*} \subseteq \mathfrak{M}^{a}$. Since $\tilde{\mathfrak{P}} \subseteq \mathfrak{P}^{*}$, we get $\tilde{\mathfrak{P}} \subseteq \mathfrak{M}^{a}$. Let $\mathfrak{P}_{0}=\tilde{\mathfrak{P}}^{G^{-1}}, \mathfrak{P}_{1}=\mathfrak{P}^{* G^{-1}}$. Then $\mathfrak{P}_{0}$ is a 3 -subgroup of $\mathfrak{M}$ and $\mathfrak{P}_{1}$ is a $S_{3}$-subgroup of $N\left(\mathfrak{P}_{0}\right)$ which is contained in $\mathfrak{M}$. This violates Lemma 9.20, since $\Re_{3} \subset \widetilde{\mathscr{R}}_{3}$. Hence, (a) holds.

Since $\mathfrak{B} \leqq \Re_{3}$, it follows from Lemma 9.16 (ii) that $\langle I\rangle$ is a $S_{2}$ subgroup of $\Re_{3} C\left(\Re_{3}\right)$. Thus, $\Re_{3} C\left(\Re_{3}\right)$ has a normal 2-complement. We assume without loss of generality that $I$ normalizes $\tilde{\Omega}_{3}$. Let $\widehat{\Re}_{3} / \Omega_{3}$ be a chief factor of $\tilde{\Re}_{3}\langle I\rangle$. Hence, $\hat{\Re}_{3}=\Re_{3} \times \bar{\Re}_{3}$, where $\left|\bar{\Re}_{3}\right|=3$. This implies that $C_{\hat{\Re}_{3}}(P)$ contains an elementary subgroup of order 27. Let $\mathfrak{S}^{G}$ be a $S_{3}$-subgroup of $\mathbb{S}$ containing $\widetilde{\Re}_{3}$, and let $\mathfrak{U} \in \mathscr{U}\left(\mathfrak{P}^{G}\right)$. Then $\boldsymbol{C}(\mathfrak{U}) \cap \boldsymbol{C}_{{\widetilde{\Re_{3}}}_{3}}(P)$ is noncyclic, and any noncyclic subgroup of $\boldsymbol{C}(\mathfrak{U}) \cap \boldsymbol{C}_{{\widetilde{\Re_{3}}}_{3}}(P)$ of order 9 may play the role of $\mathfrak{2}^{*}$ in (b).

Let $\Re$ be a maximal 2,3-subgroup of $(8)$ which contains a $S_{2,3^{-}}$ subgroup of $N(\overline{\mathfrak{P}})$. By (b), $\Re$ contains an element of $\mathscr{D}$. Assume that $\Re$ contains a noncyclic abelian subgroup of order 8 . Then by Lemma 9.17, $O_{2}(\Re) \neq 1$. By Lemma 9.16 (ii), we get $\left|Z\left(O_{2}(\Re)\right)\right|=2$. Clearly, $\Re$ is a $S_{2,3}$-subgroup of $C\left(Z\left(O_{2}(\Re)\right)\right)$, and so it contains an 
element $\mathfrak{D}$ of $\mathscr{D}$ and one of $\mathscr{U}(2)$. Applying Lemma 7.5, we get a conjugate $\mathfrak{M}^{G}$ of $\mathfrak{M}$ containing $\mathfrak{D}$ and a $S_{2}$-subgroup of $\mathfrak{R}$. By Lemma 7.5 (f), $\quad Z\left(O_{2}(\Re)\right) \subseteq C(\mathfrak{D}) \cap O_{2}\left(\mathfrak{M}^{G}\right)$. By Lemma 9.16 (ii), the last group is of order 2, and so equals $\boldsymbol{Z}\left(\boldsymbol{O}_{2}(\Re)\right)$. Hence, $\boldsymbol{Z}\left(\boldsymbol{O}_{2}(\Re)\right)=\boldsymbol{Z}\left(\boldsymbol{O}_{2}\left(\mathfrak{M}^{G}\right)\right)$, and so $\mathfrak{R} \subseteq \mathfrak{M}^{G}$. This violates (a).

Suppose $\mathbb{F}$ is an abelian subgroup of $\Re_{3}$ of order 27. Then there is an element $E$ in $\left[F\right.$ of order 3 such that $\boldsymbol{C}(E) \cap \boldsymbol{O}_{2}(\Re)$ contains a noncyclic abelian subgroup of order 8. This violates (c) and establishes $(\mathrm{d})$.

(e) is an immediate consequence of (d).

Lemma 9.22. $\Re_{2}-\Re_{0}$ contains an involution.

Proof. Suppose false. By a result of Glauberman [16], $\Re_{2}$ contains an involution $J$ such that $J=I^{G} \neq I$. Since the lemma is false, $J \in \mathfrak{R}_{0}$. Let $\mathfrak{I}=C_{\mathscr{\Re}_{0}}(J)$. Then $\mathfrak{I}$ is generated by involutions, and $\mathfrak{I} \subseteq \mathfrak{M}^{G}=C(J)$. Since the lemma is false, $\mathfrak{I} \subseteq \Re_{0}^{\mathfrak{x}}$. In particular, $I \in\left(\Re_{0}^{i}\right)^{\prime}$. Hence, $\left(\Re_{0}^{G}\right)^{\prime}=\langle J\rangle=\langle I\rangle$, a contradiction.

Lemma 9.23. The 3-length of $\mathfrak{M}$ is 1 .

Proof. Suppose false. By Lemma 9.21(e), it follows that $\Omega_{3}^{*}$ is elementary of order 9. Consider $\Omega^{*} /\langle I\rangle$. Since $\Omega^{*} \cap \Omega_{0}=\langle I\rangle$ by (9.25), it follows that $\Re_{3}^{*}\langle I\rangle \mid\langle I\rangle=\boldsymbol{F}\left(\Re^{*} /\langle I\rangle\right)$. This implies that $\AA^{*} /$ $\langle I\rangle$ contains a quaternion subgroup $\mathfrak{Q} /\langle I\rangle$. Thus, $\mathfrak{Q}$ is not of maximal class, since no group of maximal class and order 16 has a quaternion factor group. Hence, $\mathfrak{Q}$ contains a noncyclic abelian subgroup of order 8. This violates Lemma 9.21 (c) with $\Re_{3}^{*}$ in the role of $\tilde{\mathfrak{B}}$.

Lemma 9.24. Each involution $J$ of $\Re_{2}-\Re_{0}$ normalizes a $S_{3}$ subgroup of $\Re$.

Proof. Since $J \notin \Re_{0}$, Lemma 5.36 implies that $J$ inverts an element $P$ of $\Re$ of order 3 . Let $\mathfrak{S}=C_{\Re(T)}\left(\right.$ Suppose $\mathfrak{S} \cap \Omega_{0}=\langle I\rangle$. Then since $\Re_{3} \Re_{0} \triangleleft \Re$, it follows that $\mathfrak{E}$ is 3 -closed. Let $\mathfrak{F}_{3}$ be the $S_{3}$-subgroup of $\mathfrak{E}^{5}$. Thus, $C_{3}$ is noncyclic. Since $N\left(\widetilde{G}_{3}\right) \cap \Re_{0}=\langle I\rangle, N_{\Omega_{(}}\left(\mathfrak{S}_{3}\right)$ contains a $S_{3}$-subgroup of $\Omega$ as a normal subgroup. Since $J \in N\left(C_{3}\right)$, we are done.

We may assume that $\mathfrak{\complement} \cap \Re_{0} \supset\langle I\rangle$. Hence, $\sqrt{ } \cap \Re_{0}=\mathfrak{Z}$ is a quaternion group. Let $\tilde{\mathfrak{P}}$ be a $S_{3}$-subgroup of $\sqrt{5}$. Thus, $\tilde{\mathfrak{P}} \mathfrak{Q}=\boldsymbol{O}^{3}(\mathfrak{S})$

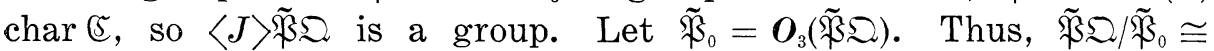

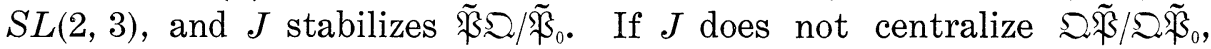

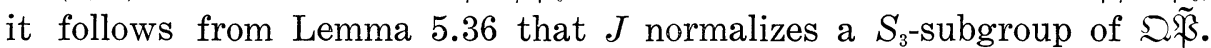
Suppose $J$ centralizes $\tilde{\mathfrak{P}} \mathfrak{\Omega} / \mathfrak{\mathfrak { R }} \tilde{\mathfrak{P}}_{0}$. Then $\langle J\rangle \tilde{\mathfrak{P}} \mathfrak{Q} / \mathfrak{Q} \tilde{\mathfrak{P}}_{0}$ is a cyclic group of order 6 , so $J$ centralizes $\mathfrak{\Omega} / \mathfrak{Q}^{\prime}$. This implies that $|\boldsymbol{C}(J) \cap \mathfrak{\Omega}| \geqq 4$, so that $\langle J, \mathfrak{\Omega}\rangle$ contains a noncyclic abelian subgroup of order 8. Since $\langle J, \mathfrak{Q}\rangle \subseteq N(\langle P\rangle)$, Lemma 9.21 (c) is violated. We conclude that $J$ 
normalizes a $S_{3}$-subgroup of $\tilde{\mathfrak{\beta}} \Omega$. We assume without loss of generality that $J$ normalizes $\tilde{\mathfrak{R}}$. Since $N(\tilde{\mathfrak{P}}) \cap \Re_{0}=\langle I\rangle$, it follows that $N_{\mathscr{\Re}}(\tilde{\mathfrak{P}})$ contains a $S_{3}$-subgroup of $\Omega$ as a normal subgroup. The proof is complete.

Lemma 9.25. (a) If $T$ is an involution of $B$, $C(T)$ contains a noncyclic abelian subgroup of order 8.

(b) If the width of $\Re_{0}$ is 2, then for each involution $T$ of (S), $|C(T)|_{3} \leqq 9$.

Proof. (a) By Lemma 5.38, $C(T)$ contains an element $\mathfrak{U}$ of $\mathscr{U}(2)$. If $T \notin \mathfrak{M}$, then $\langle\mathfrak{M}, T\rangle$ is a noncyclic abelian subgroup of order 8 which is contained in $C(T)$. Suppose $T \in \mathfrak{H}$. Since $\mathscr{S}_{\text {cres }_{3}}(2) \neq \varnothing, C(T)$ contains an element of $\mathscr{S}_{\text {in }_{3}}(2)$ by Lemma 0.8.9.

Suppose (b) is false, and $T$ is an involution of (5) with $|\boldsymbol{C}(T)|_{3} \geqq 27$. Let $\subseteq$ be a maximal 2, 3-subgroup of (S) which contains a $S_{2,3}$-subgroup of $\boldsymbol{C}(T)$. By Lemma 5.38, $\subseteq$ contains an element $\mathfrak{u}$ of $\mathscr{U}(2)$. Let $\mathfrak{S}_{p}$ be a $S_{p}$-subgroup of $\mathfrak{S}, p=2,3$. We assume without loss of generality that $\mathfrak{S}_{2} \subseteq \Re_{2}$.

\section{Case 1. $\quad \boldsymbol{O}_{3}(\mathfrak{S}) \neq 1$.}

Since $\mathfrak{U} \in \mathscr{E}(2), \mathfrak{U}$ centralizes $\boldsymbol{O}_{3}(\Im)$. Since $\mathfrak{U}$ contains a conjugate of $I$, it follows that $\left|O_{3}(\Im)\right| \leqq 9$. Suppose $\left|O_{3}(\Im)\right|=9$. Then $O_{3}(\Im)$ is conjugate to $\mathfrak{B}$, since $\mathfrak{B}$ is a $S_{3}$-subgroup of $\mathfrak{M}$. But then Lemma 9.16 (ii) is violated. Hence, $\left|O_{3}(\Im)\right|=3$.

Since $\mathfrak{U}$ centralizes $\boldsymbol{O}_{3}(\mathfrak{S}), \boldsymbol{O}_{3}(\mathfrak{S})$ is conjugate to a subgroup of $\mathfrak{B}$. By Lemma $9.21(b), C\left(\boldsymbol{O}_{3}(\mathfrak{S})\right)$ contains an elementary subgroup $\mathfrak{H}^{*}$ such that $C(A)$ contains an element of $\mathscr{C}(3)$ for each $A$ in $\mathfrak{U}^{*}$. Since $\subseteq$ is a $S_{2,3}$-subgroup of $\mathbf{N}\left(\boldsymbol{O}_{3}(\mathfrak{S})\right.$ ), we assume without loss of generality that $\mathfrak{U}^{*} \subseteq \mathfrak{S}$. By Lemma 9.16 (i), $\mathfrak{X}^{*} \in \mathscr{D}$. Now Lemma 9.17 yields $\boldsymbol{O}_{2}(\mathfrak{S}) \neq$ 1. Since $\mathfrak{U}^{*} \subseteq \subseteq$, Lemma 9.16 (ii) forces $\left|\boldsymbol{Z}\left(\boldsymbol{O}_{2}(\Im)\right)\right|=2$, and forces $\boldsymbol{Z}\left(\boldsymbol{O}_{2}(\mathfrak{S})\right)$ to be a maximal characteristic abelian subgroup of $\boldsymbol{O}_{2}(\widetilde{S})$. Since $\left|\boldsymbol{O}_{3}(\mathfrak{S})\right|=3$, it follows that $\left|\boldsymbol{O}_{2}(\mathfrak{S})\right|>2$. Hence, $\boldsymbol{O}_{2}(\mathfrak{S})$ is extra special. Thus, $\boldsymbol{O}_{2}(\mathfrak{S})^{\prime}$ is of order 2 and is normalized by every element of $\mathscr{U}\left(\Re_{2}\right)$. Hence, every element of $\mathscr{U}\left(\Omega_{2}\right)$ is contained in $\widetilde{S}_{2}$. Thus, $I$ centralizes $\boldsymbol{O}_{3}(\mathfrak{S})$. Since $I \in \boldsymbol{Z}\left(\mathfrak{S}_{2}\right)$, we get $I \in \boldsymbol{O}_{2}(\mathfrak{S})$, so that $\langle I\rangle=$ $\boldsymbol{O}_{2}(\subseteq)^{\prime}$. Hence, $\subseteq \subseteq \mathfrak{M}$, against $|\subseteq|_{3} \geqq 27$ and $|\mathfrak{M}|_{3}=9$.

Case 2. $\boldsymbol{O}_{3}(\mathfrak{S})=1$.

Since $|\mathfrak{S}|_{3} \geqq 27$, it follows that $m\left(O_{2}(\mathfrak{S})\right) \geqq 6$. Since the width of $\Re_{0}$ is 2 , it follows that $\Re_{2}$ has no elementary subgroup of order $2^{6}$. Thus, $\boldsymbol{O}_{2}(\mathfrak{S})$ is not elementary.

Now $\subseteq$ is clearly not contained in any conjugate of $\mathfrak{M}$, since $|\mathfrak{S}|_{3}>|\mathfrak{M}|_{3}$. Since $\langle I\rangle=Z\left(\Re_{2}\right)$, it follows that $\mathfrak{S}$ is not 2-closed. 
Since $\left|\Re_{2}\right| \leqq 2^{8}$, we get $\left|O_{2}(\mathfrak{S})\right|=2^{7}$. Hence, $\left|O_{2}(\mathfrak{S})\right|=2^{7}$, so that $D\left(O_{2}(\Im)\right)$ is a subgroup of order 2 and $\mathfrak{S}_{2}$ is of order $2^{8}$. Hence, $\mathfrak{S}_{2}=\Re_{2}$ and $\boldsymbol{D}\left(\boldsymbol{O}_{2}(\mathfrak{S})\right)=\langle I\rangle$. This shows that $\mathfrak{S} \subseteq \mathfrak{M}$. This contradiction completes the proof.

LEMma 9.26. If $\tilde{\mathfrak{P}}$ is any subgroup of $\Re_{3}$ of order 3 , then

(a) $\Re_{0} \cap \boldsymbol{C}(\tilde{\mathfrak{\beta}})$ is either $\langle I\rangle$ or a quaternion group;

(b) if $\tilde{\mathfrak{\beta}} \nsubseteq \boldsymbol{Z}\left(\AA_{3}\right)$, then $\Re_{0} \cap \boldsymbol{C}(\tilde{\mathfrak{\beta}})$ is quaternion.

Proof. (a) Suppose $\Re_{0} \cap \boldsymbol{C}(\tilde{\mathfrak{P}}) \supset\langle I\rangle$. Then $\Re_{0} \cap \boldsymbol{C}(\tilde{\mathfrak{P}})$ is extra special and does not contain a noncyclic abelian subgroup of order 8 . Thus, $\Omega_{0} \cap \boldsymbol{C}(\tilde{\mathfrak{R}})$ is either dihedral or quaternion. Now $\boldsymbol{C}_{\Re_{3}}(\tilde{\mathfrak{R}})$ contains an elementary subgroup $\mathbb{F}$ of order 9 with $\tilde{\mathfrak{\beta}} \subset \mathfrak{F}$. Hence, $\Re_{0} \cap \boldsymbol{C}(\tilde{\mathfrak{\beta}})$ admits (5. Since no element of $\mathfrak{F}^{\#}$ centralizes a noncyclic abelian subgroup of $\Omega_{0}$ of order $8, \Omega_{0} \cap \boldsymbol{C}(\tilde{\mathfrak{R}})$ is quaternion.

(b) Let $\mathbb{F}=\left\langle\tilde{\mathfrak{P}}, Z\left(\Re_{3}\right)\right\rangle$, so that by Lemma $9.21(\mathrm{e})$, $[\mathfrak{F}$ is elementary of order 9 and $₹ \triangleleft \Re_{3}$. It follows that the three subgroups of $\&$ of order 3 which are distinct from $\boldsymbol{Z}\left(\Omega_{3}\right)$ are conjugate in $\Omega_{3}$. We can choose $E$ in $\left(F *\right.$ such that $\Re_{0} \cap C(E)$ is not centralized by $\boldsymbol{Z}\left(\Re_{3}\right)$. By (a), $\Re_{0} \cap \boldsymbol{C}(E)$ is a quaternion group; so (b) holds.

LEMMA 9.27. $\Omega_{2}^{*}$ is a four-group.

Proof. Suppose false. By Lemma 9.22, $\Re_{2}-\Re_{0}$ contains an involution $J$. By Lemma 9.24, $J$ normalizes a $S_{3}$-subgroup of $\Re$. Thus, we can choose $M$ in $\mathfrak{M}$ such that $J^{M}=T_{0}$ normalizes $\AA_{3}$. Since $\Re_{3}$ permutes transitively by conjugation the $S_{2}$-subgroups of $\mathscr{R}$, we may choose $K$ in $\Re_{3}$ such that $T=T_{0}^{K}$ lies in $\Omega_{2}$. Thus, $T \in N\left(\Omega_{3}\right) \cap \Re_{2}$.

By Lemma 9.23, $\Re_{3}^{*}=\Re_{3}$. Thus, $T \in \Re_{2}^{*}$. If $\langle T, I\rangle=\Re_{2}^{*}$, we are done, so suppose $\langle T, I\rangle \subset \Re_{2}^{*}$. Let $\mathfrak{F}$ be a subgroup of $\Re_{2}^{*}$ of order 8 which contains $\langle T, I\rangle$. By Lemma 9.19, $\mathfrak{F}$ is dihedral of order 8 . Let $\mathfrak{F}_{0}, \mathfrak{F}_{1}$ be the four-subgroups in $\mathfrak{F}_{\text {. }}$.

Suppose $\mathfrak{X}$ is a subgroup of $\Re_{3}$ of order 3 which admits $\mathfrak{F}$ and that $C(\mathfrak{X}) \cap \Re_{0}$ is a quaternion group. Hence, $\boldsymbol{C}_{\mathfrak{M}}(\mathfrak{X})$ contains a normal quaternion subgroup and $S_{2}$-subgroups of $N_{\mathfrak{M}}(\mathfrak{X})$ are of order at least $2^{5}$. Thus, $N_{\mathfrak{M}}(\mathfrak{X})$ contains a noncyclic abelian subgroup of order 8 . This is impossible, by Lemma $9.21(\mathrm{c})$. Hence, $C_{\mathfrak{\Re}_{0}}(\mathfrak{X})=\langle I\rangle$, by Lemma 9.26 (a).

By Lemma 9.26 (b) and the preceding paragraph, it follows that $\widetilde{\mho}$ normalizes no noncentral subgroup of $\Omega_{3}$ of order 3 .

Suppose $\Omega_{3}$ is nonabelian. Then $\widetilde{F}$ normalizes $\Omega_{1}\left(\Re_{3}\right)$, a group of exponent 3. Since $\langle I\rangle$ centralizes $\Re_{3}$, it follows that $\Omega_{1}\left(\Re_{3}\right) \mathfrak{F}$ is supersolvable. Thus, $\Omega_{1}\left(\Re_{3}\right) \mathfrak{F}$ contains a normal subgroup of order 9 , so $\mathfrak{F}$ normalizes a noncentral subgroup of $\Omega_{3}$ of order 3 . This contradicts the preceding paragraph, so we conclude that $\Omega_{3}$ is abelian, $\Omega_{3}=\mathfrak{B}$. 
Let $\mathfrak{F}_{i}=\left\langle J_{i}, I\right\rangle, i=0,1$. If both $J_{0}$ and $J_{1}$ invert $\Re_{3}$, then $J_{0} J_{1}$ centralizes $\Re_{3}$, so $J_{0} J_{1} \in\langle I\rangle$. This is not the case, since $\mathfrak{F}_{0} \widetilde{F}_{1}$ is of order 8. Thus, we may assume notation is chosen so that $J_{0}$ centralizes $\mathfrak{X}_{0}$ and inverts $\mathfrak{X}_{1}$. Here, $\left|\mathfrak{X}_{i}\right|=3$, and $\Re_{3}=\mathfrak{X}_{0} \times \mathfrak{X}_{1}$. Since $\mathfrak{R}_{0} \cap \boldsymbol{C}\left(\mathfrak{X}_{i}\right)=$ $\langle I\rangle$, for $i=0,1$, the width of $\Omega_{0}$ is 2 .

Let $\&$ be a $S_{2,3}$-subgroup of $N\left(\Omega_{3}\right)$ which contains $\Omega_{2}^{*}$. Since $\Re_{3} \subset \mathfrak{P}$, we get $\left|\Re_{3}\right|=9<|\mathfrak{F}|_{3}$. By Lemma $9.16(\mathrm{ii}),\left|C\left(\Re_{3}\right)\right|_{2}=2 . \quad$ By Lemma 9.20, we get that $\mathbb{F}$ is 3 -closed. Let $\mathfrak{F}_{3}=\boldsymbol{O}_{3}(\mathfrak{F}) \supset \Re_{3}$.

Let $F_{0}, F_{1}, F_{2}$ be the three involutions of $\mathfrak{F}_{0}$, and set

$$
3^{f_{i}}=\left|\mathfrak{F}_{3} \cap C\left(F_{i}\right)\right|, i=0,1,2 \text {. }
$$

By Lemma $9.25(\mathrm{~b})$, we have $f_{i} \leqq 2$. Since $\mathfrak{F}_{3} \cap C\left(\mathfrak{\mho}_{0}\right)=\mathfrak{X}_{0}$, a formula of Wielandt [44] yields

$$
\left|\mathfrak{F}_{3}\right|=3^{f_{0}+f_{1}+f_{2}-2} \leqq 3^{4}
$$

Since the dihedral group $\mathfrak{F}$ is faithfully represented on $\mathfrak{F}_{3} / \mathscr{\Re}_{3}$, it follows that $\left|\mathfrak{F}_{3}\right|=3^{4}$.

Let $\mathfrak{D}$ be a $S_{2,3}$-subgroup of $N\left(\widetilde{夭}_{3}\right)$. Let $\mathfrak{D}_{p}$ be a $S_{p}$-subgroup of $\mathfrak{D}$, with $\mathfrak{R}_{2}^{*} \subseteq \mathfrak{D}_{2}$. By the formula of Wielandt [44] applied to $\mathfrak{F}_{0}$ acting on $\boldsymbol{O}_{3}(\mathfrak{D})$, we get $\boldsymbol{O}_{3}(\mathfrak{D})=\mathfrak{F}_{3}$. If $\mathfrak{D}_{3}=\mathfrak{F}_{3}$, then $\mathfrak{F}_{3}$ is a $S_{3}$ subgroup of $\mathbb{S}$. But the center of $\mathfrak{F}_{3}$ contains $\Omega_{3}=\mathfrak{B}$ by Lemma 9.20 , and hence is noncyclic. This contradicts hypothesis (iii) of Theorem 9.1. Therefore $\mathfrak{D}_{3} \supset \mathfrak{F}_{3}$ and $\mathfrak{D}$ is not 3-closed.

By Lemma $9.25(b)$, we get $O_{2}(\mathfrak{D})=1$. Since $\mathfrak{D}$ is quite obviously contained in no conjugate of $\mathfrak{M}$, Lemma 9.18 implies that $\mathfrak{D}$ contains no noncyclic abelian subgroups of order 8 . Thus, $\mathfrak{D}_{2}$ is of maximal class. Hence, $\langle I\rangle=\boldsymbol{Z}\left(\mathfrak{D}_{2}\right)$, so $\mathfrak{D}_{2}=\Re_{2}^{*}$ is of order at most 16 . Suppose $\left|\mathfrak{D}_{2}\right|=16$. Since $\boldsymbol{O}_{2}(\mathfrak{D})=1$, and $\mathfrak{D}$ is not 3 -closed, it follows that $\boldsymbol{O}_{3,2}(\mathfrak{D}) \cap \mathfrak{D}_{2}$ is a quaternion group. But then $\mathfrak{M}$ covers $\mathfrak{D} / \boldsymbol{O}_{3}(\mathfrak{D})$. This is not the case, since $O_{3}(\mathfrak{D})$ contains a $S_{3}$-subgroup of $\mathfrak{M}$, and since 3|| $\mathfrak{D}: \boldsymbol{O}_{3}(\mathfrak{D}) \mid$. Hence, $\mathfrak{D}_{2}=\mathfrak{F}$ is dihedral of order 8 . Let $\widetilde{D}_{2}=$ $\boldsymbol{O}_{3,2}(\mathfrak{D}) \cap \mathfrak{D}_{2}$. Thus, $\tilde{\mathfrak{D}}_{2}$ is a four-group and $\mathfrak{D} / \boldsymbol{O}_{3}(\mathfrak{D}) \cong \Sigma_{4}$.

Since $\mathfrak{D} / O_{3}(\mathfrak{D}) \cong \Sigma_{4}$, some chief factor of $\mathfrak{D}$ is of order $3^{3}$. Thus, $\boldsymbol{O}_{3}(\mathfrak{D})$ is necessarily elementary, and elements of $\mathfrak{D}_{3}-\boldsymbol{O}_{3}(\mathfrak{D})$ induce automorphisms of $\boldsymbol{O}_{3}(\mathfrak{D})$ with minimal polynomial $(x-1)^{3}$. Hence, $\boldsymbol{O}_{3}(\mathfrak{D})=J\left(\mathfrak{D}_{3}\right) \boldsymbol{O}_{3}(\mathfrak{D})=J\left(\mathfrak{D}_{3}\right) \operatorname{char} \mathfrak{D}_{3}$, so $\mathfrak{D}_{3}$ is a $S_{3}$-subgroup of $\mathbb{S}$. This is not the case, since $Z\left(\mathfrak{D}_{3}\right)$ is noncyclic. The proof is complete.

LEMMA 9.28. If the width of $\Re_{0}$ exceeds 2, then $I$ is the only conjugate of $I$ in $\Re_{0}$.

Proof. Suppose $T=I^{G} \neq I, T \in \Re_{0}$. Then $C(T) \cap \Re_{0} \subseteq C(T)=\mathfrak{M}^{G}$. By Lemma 9.27, $C(T) \cap \Re_{0} \cap \Re_{0}^{G}$ is of index at most 2 in $C(T) \cap \Re_{0}$. 
Since $C_{\Re_{0}}(T)$ is of index 2 in $\Re_{0}$, we get $\left|\Re_{0}: C(T) \cap \Re_{0} \cap \Re_{0}^{G}\right| \leqq 4$. Since the width of $\Re_{0}$ is at least 3 , it follows that $C(T) \cap \Re_{0} \cap \Re_{0}^{G}$ is nonabelian. Hence, $\langle I\rangle=\left(C(T) \cap \Re_{0} \cap \Re_{0}^{G}\right)^{\prime}=\langle T\rangle$. This contradiction completes the proof.

LEMMA 9.29. $\Re_{3}=\mathfrak{B}$ is of order 9 .

Proof. Suppose false. By Lemma 9.21(e), $\Omega_{3}$ is nonabelian of order 27. Since $\Omega_{3}$ is faithfully represented on $\Omega_{0}$, the width of $\Omega_{0}$ is at least 3. By a result of Glauberman [14], $\Re_{2}$ contains a conjugate $T$ of $I$ distinct from $I, T=I^{G} \neq I$. By Lemma 9.28, $T \in \Re_{2}-\Re_{0}$, so by Lemma 9.24 , we may assume that $T \in \mathfrak{R}_{2}^{*}$. Thus, by Lemma 9.27, $\Re_{2}^{*}=\langle I, T\rangle$.

Since $\Re_{3}$ is nonabelian, it follows that $\mathfrak{X}_{1}=\Re_{3} \cap C(T)$ is of order 3 . By Lemma 1.3 of [17], $\Re_{3}$ has a subgroup $\mathfrak{X}_{0}$ of order 3 which centralizes $\mathfrak{X}_{1}$ and is inverted by $T$. Let $\mathfrak{X}=\mathfrak{X}_{0} \times \mathfrak{X}_{1}$ and let $\mathfrak{X}_{2}, \mathfrak{X}_{3}$ be the remaining subgroups of $\mathfrak{X}$ of order 3 .

Suppose $C\left(\mathfrak{X}_{0}\right) \cap \Re_{0} \supset\langle I\rangle$. By Lemma 9.26(a), $C\left(\mathfrak{X}_{0}\right) \cap \Re_{0}=\Omega$ is a quaternion group. Since $C(\mathfrak{X}) \cap \Re_{0}=\langle I\rangle$, it follows that $\mathfrak{X}_{1}$ is faithfully

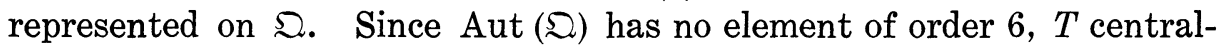

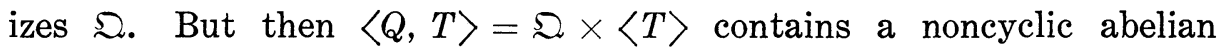
subgroup of order 8 . This violates Lemma 9.21 (c) with $\mathfrak{X}_{0}$ in the role of $\mathfrak{\Re}$. Hence, $\boldsymbol{C}\left(\mathfrak{X}_{0}\right) \cap \mathfrak{\Re}_{0}=\langle I\rangle$.

Since $C\left(\mathfrak{X}_{0}\right) \cap \Re_{0}=\langle I\rangle$, the width of $\Re_{0}$ is at most 3 . By Lemma $9.26(\mathrm{~b})$, we get $\mathfrak{X}_{0}=\boldsymbol{Z}\left(\Re_{3}\right)$. Thus, if we set $\mathfrak{\Omega}_{i}=\Re_{0} \cap \boldsymbol{C}\left(\mathfrak{X}_{i}\right), i=1,2,3$, then by Lemma 9.26, it follows that each $\mathfrak{\Omega}_{i}$ is quaternion. Hence, $\Re_{0}$ is the central product of $\mathfrak{\Omega}_{1}, \mathfrak{\Omega}_{2}, \mathfrak{\Omega}_{3}$. Since $T$ centralizes $\mathfrak{X}_{1}$ and interchanges $\mathfrak{X}_{2}$ and $\mathfrak{X}_{3}$, it follows that $T$ normalizes $\mathfrak{\Omega}_{1}$ and interchanges $\mathfrak{\Omega}_{2}$ and $\mathfrak{\Omega}_{3}$. Since $\mathfrak{X}_{0}$ is faithfully represented on $\mathfrak{\Omega}_{1}$, it follows that $\mathfrak{D}_{1} \mathfrak{X}_{0}\langle T\rangle \cong G L(2,3)$. Thus, we can choose generators $A_{i}, B_{i}$ for $\mathfrak{\Omega}_{i}$ such that $A_{1}^{T}=B_{1}, A_{2}^{T}=A_{3}, B_{2}^{T}=B_{3}$. It follows that $C(T) \cap \Omega_{0}=$ $\left\langle A_{2} A_{2}, B_{2} B_{3}, I\right\rangle$, an elementary group of order 8. Let $\mathfrak{F}=C(T) \cap \Re_{2}=$ $\langle T\rangle \times C(T) \cap \Re_{0}$. It now follows that $\tilde{\Re}_{2}=N_{\Re_{2}}(\mathfrak{F})=\left\langle\Omega_{2}, \Omega_{3}, A_{1} B_{1}, T\right\rangle$, a group of index 2 in $\Re_{2}$. Since $Z\left(\tilde{\Re}_{2}\right)=\langle I\rangle$, it follows that $\tilde{\Omega}_{2}$ is a

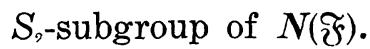

Now $T=I^{a}$, so $\mathfrak{F} \subseteq \mathfrak{M}^{a}$. By symmetry, $N(\mathfrak{F}) \cap \mathfrak{M}^{G}$ contains a $S_{2}$-subgroup of $N(\mathfrak{F})$. This implies that $O_{2}(N(\mathfrak{F})$ ) centralizes both $T$ and $I$. Hence, $\boldsymbol{O}_{2}(\boldsymbol{N}(\mathfrak{\mho}))=\mathfrak{F}$.

Now $\widetilde{\Re}_{2}$ permutes transitively the elements of $\left(\mathfrak{F} \cap \Re_{0}\right) T$, so $\mathfrak{S}=$ $\left\{\left(\mathfrak{F} \cap \Re_{0}\right) T, I\right\}$ is the set of all the elements of $\mathfrak{F}$ which are conjugate to $I$ in $\mathbb{S}$. Since $N(\mathfrak{F}) \cap \mathfrak{M}^{G}$ normalizes $\mathfrak{S}$ but does not centralize $I$, it follows that $N(\mathfrak{F})$ permutes $\mathfrak{S}$ transitively.

Since $N(\mathfrak{F})$ is transitive on $\mathfrak{S}$, it follows that $N(\mathfrak{F})=9 \cdot|N(\mathfrak{F}) \cap \mathfrak{M}|$. Since $\boldsymbol{C}(\mathfrak{F})=\boldsymbol{C}_{\mathfrak{M}}(\mathfrak{F})$, it follows that $\mathfrak{F}=\boldsymbol{C}(\mathfrak{F})$. Since $T$ centralizes $\mathfrak{X}_{1}$, 
it follows that $\mathfrak{X}_{1}$ normalizes $\Re_{0} \cap C(T)$, so normalizes $\mathfrak{F}=\langle T\rangle \times \Re_{0} \cap$ $C(T)$. But it now follows that 27||$N(\mathfrak{F}) \mid$. Since $\mathfrak{F}$ is elementary of order $2^{4}$, Aut (F) has no subgroup of order 27. This violates the equality $\mathfrak{F}=\boldsymbol{C}(\mathfrak{F})$, and the proof is complete.

Lemma 9.30. If $T$ is any involution of (S), then $|C(T)|_{3} \leqq 9$.

Proof. Since $\left|\Re_{2}: \Re_{0}\right|=2$, Lemma 5.38 implies that every involution of $B S$ is conjugate to an involution of $\Re_{0}$. Thus, we may assume that $T \in \mathfrak{\Re}_{0}$. If $T \sim I$, we are done by Lemma 9.29 , so from now on we suppose $T \nsim I$.

Let $\mathfrak{U} \in \mathscr{U}\left(\Re_{2}\right)$, and let $\widetilde{\Re}_{0}=\Re_{0} \cap C(\mathfrak{U})$. Thus, $\widetilde{\Omega}_{0}$ is of index 2 in $\Re_{0}$. Since $\Re_{3}$ has no fixed points on $\Re_{0} /\langle I\rangle$, Lemma 5.38 implies that for some $X$ in $\Re_{3}, T^{X} \in \tilde{\Re}_{0}$. Thus, we assume without loss of generality that $T \in \widetilde{\Re}_{0}$.

We argue that $C_{\mathfrak{M}}(T)$ contains a $S_{2}$-subgroup of $C(T)$. This is clear if $\left|\mathfrak{M}: C_{\mathfrak{M}}(T)\right|_{2}=2$, since $T \nsim I$. So suppose $\left|\mathfrak{M}: C_{\mathfrak{M}}(T)\right|_{2}=4$. In this case, $\boldsymbol{C}_{\Re_{0}}(T)$ is a $S_{2}$-subgroup of $\boldsymbol{C}_{\mathfrak{M}}(T)$. Since $\langle I\rangle=C_{\Re_{0}}(T)^{\prime}$ char $C_{\Re_{0}}(T)$, it follows that $C_{\mathscr{\Re}_{0}}(T)$ is a $S_{2}$-subgroup of $C(T)$.

Let $\Re$ be a $S_{2,3}$-subgroup of $C(T)$ which contains $C_{\Re}(T)$. Suppose $\boldsymbol{O}_{3}(\mathfrak{R}) \neq 1$. Since $\mathfrak{U} \subseteq \mathfrak{R}, \mathfrak{U}$ centralizes $\boldsymbol{O}_{3}(\mathfrak{R})$, so $\boldsymbol{O}_{3}(\mathfrak{R}) \subseteq \mathfrak{M}$. Since no element of $\Omega_{3}^{*}$ centralizes a four-subgroup of $\Re_{0}$ by Lemma 9.26 (a), we conclude that $O_{3}(\Re)=1$.

Since $\boldsymbol{O}_{3}(\Re)=1$ and since $\Re \cap \mathfrak{M}$ contains a $S_{2}$-subgroup of $C(T)$, it follows that $I \in \boldsymbol{Z}\left(\boldsymbol{O}_{2}(\Re)\right)$. Suppose $X$ is a 3-element of $\Re$ and $\mathfrak{X}$ centralizes $I$. Then $X \in C(\langle T, I\rangle)$, so $X=1$ by Lemma 9.26 (a). Thus, a $S_{3}$-subgroup $\Re_{3}$ of $\Re$ is faithfully represented on $Z\left(O_{2}(\Re)\right.$ ).

Let $\Omega_{1}\left(\boldsymbol{Z}\left(\boldsymbol{O}_{2}(\Re)\right)\right)=\mathfrak{Y}_{1} \times \mathfrak{Y}_{2}$, where $\mathfrak{Y}_{1}=\Omega_{1}\left(\boldsymbol{Z}\left(\boldsymbol{O}_{2}(\Re)\right)\right) \cap \boldsymbol{C}\left(\mathfrak{R}_{3}\right)$, and $\mathfrak{Y}_{2}=\left[\Omega_{1}\left(\boldsymbol{Z}\left(\boldsymbol{O}_{2}(\mathfrak{R})\right)\right), \mathfrak{R}_{3}\right]$. Thus, $T \in \mathfrak{Y}_{1}$ and $\mathfrak{R}_{3}$ is faithfully represented on $\mathfrak{Y}_{2}$. Hence, $m\left(\boldsymbol{Z}\left(\boldsymbol{O}_{2}(\Re)\right)=m\left(\mathfrak{Y}_{1}\right)+m\left(\mathfrak{Y}_{2}\right) \geqq 7\right.$. Thus, $\Re_{0}$ has an elementary subgroup of order $2^{6}$, by Lemma 9.27 . This is impossible, since the width of $\Re_{0}$ is at most 4 . The proof is complete.

\section{LEMMA 9.31. $\mid$ \&S $\left.\right|_{3}>3^{4}$.}

Proof. Let $\mathfrak{X}$ be a subgroup of $\Omega_{3}$ of order 3 such that $\Re_{0} \cap C(\mathfrak{X})=$ $\mathfrak{Q}$ is quaternion. Let $\mathfrak{C}$ be a $S_{2,3}$-subgroup of $\boldsymbol{C}(\mathfrak{X})$ which contains $\Re_{3} \Omega$. Since $\Re_{3}=\mathfrak{B} \in \mathscr{D}$, it follows that $\Re_{3}$ centralizes $\boldsymbol{Z}\left(\boldsymbol{O}_{2}(\mathfrak{S})\right)$. Since $\langle I\rangle$ is a $S_{2}$-subgroup of $C\left(\Re_{3}\right)$, it follows that $O_{2}(\mathfrak{C})=1$, by Lemma 9.21(a).

Since $\boldsymbol{O}_{2}(\mathbb{E})=1, \mathfrak{\Omega}$ is faithfully represented on $\boldsymbol{O}_{3}(\mathfrak{E})$, so is faithfully represented on $O_{3}(\mathfrak{E}) / \mathfrak{X}$. Hence, $\left|O_{3}(\mathfrak{E}): \mathfrak{X}\right| \geqq 9$. Since $\left[\Re_{3} \cap \boldsymbol{O}_{3}(\mathfrak{E}), \mathfrak{Q}\right] \subseteq$ $O_{3}(\mathfrak{E}) \cap \mathfrak{Q}=1$, it follows that $\Re_{3} \cap \boldsymbol{O}_{3}(\mathfrak{E})=\mathfrak{X}$. Hence, $|\mathfrak{E}|_{3} \geqq 3^{4}$. Suppose the lemma is false. Then (S contains a $S_{3}$-subgroup of $\mathbb{B}$, and $\boldsymbol{O}_{3}(\mathbb{E})$ is of order $3^{3}$, while $\mathfrak{X} \sim 3$. If $\boldsymbol{O}_{3}(\mathbb{E})$ is nonabelian, then $\mathrm{Hy}$ pothesis 9.1 is satisfied. This is not the case, so $\boldsymbol{O}_{3}(\mathbb{E})$ is elementary. Hence, $\boldsymbol{O}_{3}(\mathfrak{S})=\mathfrak{X} \times\left[\boldsymbol{O}_{3}(\mathfrak{C}), \mathfrak{\Omega}\right]$. Hence, the center of a $S_{3}$-subgroup of 
(S) is noncyclic. This is not the case. The proof is complete.

LEMma 9.32. Choose $J$ in $\Re_{2}^{*}-\langle I\rangle$. If $J$ inverts $\Re_{3}$, then $\boldsymbol{A}_{\mathscr{S}}\left(\Re_{2}^{*}\right)=\operatorname{Aut}\left(\Re_{2}^{*}\right)$.

Proof. Let $\mathfrak{X}$ be any four-subgroup of $\mathfrak{M}$ which contains $I$. We will show that

$$
\left|\boldsymbol{A}_{\mathfrak{M}}(\mathfrak{X})\right|=2 \text {. }
$$

This is clear if $\mathfrak{X} \subseteq \Re_{0}$. If $\mathfrak{X} \nsubseteq \Re_{0}$, then by Lemmas 9.27 and 9.24, we see that $\mathfrak{X}$ is conjugate to $\Re_{2}^{*}$ in $\mathfrak{M}$. Let $\mathfrak{Y}$ be a subgroup of $\Re_{3}$ such that $\mathfrak{\Omega}=\mathfrak{\Omega}_{0} \cap \boldsymbol{C}(\mathfrak{V})$ is quaternion. Since $J$ inverts $\Re_{3}, \mathfrak{Q}$ admits $\langle J\rangle \Re_{3} / \mathscr{Y}$ as a group of automorphisms. Hence, $J$ inverts an element $Q$ of $\Omega$ of order 4 . Then $J Q J=Q^{-1}$, that is, $Q^{-1} J Q=J I$, so $Q \in N_{\mathfrak{M}}\left(\Re_{2}^{*}\right)$. Thus, (9.25) holds.

Suppose that $\Omega_{2}^{*}-\langle I\rangle$ contains a conjugate $J$ of $I$. By (9.25), we can choose $M$ in $M \cap N\left(\Re_{2}^{*}\right)$ such that $M^{-1} J M=J I$. By (9.25) again, this time applied to the group $C(J)$, we can choose $M_{0}$ in $C(J)$ with $M_{0}^{-1} I M_{0}=I J$. Thus, the lemma follows in this case.

We may now assume that

$$
I \text { is the only conjugate of } I \text { in } \Re_{2}^{*} \text {. }
$$

By a result of Glauberman [16], $\Omega_{2}$ contains a conjugate $T$ of $I$ with $T \neq I$. If the width of $\Re_{0}$ exceeds 2 , then by Lemma 9.28, $T \notin \Re_{0}$, so by Lemma $9.24,(9.26)$ is violated. So suppose the width of $\Omega_{0}$ is 2. In this case, $\Re_{0}$ has exactly 18 noncentral involutions and they are permuted transitively in $\mathfrak{M}$. Since $T$ lies in no $\mathfrak{M}$-conjugate of $\Re_{2}^{*}$, Lemma 9.24 implies that $T \in \Re_{0}$. Thus, every involution of $\Re_{0}$ is conjugate to $I$ in $\$ 5$. But by Lemma 5.38, every involution of $\mathbb{B S}$ is conjugate to an element of $\Omega_{0}$. The proof is complete.

Lemma 9.33. There is a $S_{3}$-subgroup of (S) which contains $\Re_{3}$ and is normalized by $\Re_{2}^{*}$.

Proof. Let $\tilde{\mathfrak{P}}$ be a maximal element of $N\left(\Re_{2}^{*} ; 3\right)$ which contains $\Re_{3}$. Suppose by way of contradiction that $|\tilde{\mathfrak{R}}|<\left.|| \mathfrak{S}\right|_{3}$. Let $\mathbb{C}$ be a $S_{2,3}$-subgroup of $N(\tilde{\mathfrak{P}})$ which contains $\mathscr{R}_{2}^{*}$. Let $\mathfrak{S}_{p}$ be a $S_{p}$-subgroup of $\mathfrak{S}, p=2,3$, with $\Re_{2}^{*} \subseteq \mathfrak{S}_{2}$.

Suppose $\boldsymbol{O}_{2}(\mathfrak{\complement}) \neq 1$. Then since $\Re_{3} \in \mathscr{D}$, we get $Z\left(O_{2}(\mathfrak{S})\right) \sim\langle I\rangle$, so $\mathfrak{C}$ is in a conjugate of $\mathfrak{M}$. This is not the case, by Lemma 9.21(a). Clearly, the maximality of $\tilde{\mathfrak{P}}$ forces $\tilde{\mathfrak{P}}=\boldsymbol{O}_{3}(\mathfrak{E})$. Since $\boldsymbol{O}_{2}(\mathfrak{E})=1$, the proof of Lemma 9.17 implies that $\mathbb{E}$ has no noncyclic abelian subgroup of order 8. Thus, $\overline{\mathfrak{C}}=\mathbb{\mathfrak { S }} / \mathrm{O}_{3}(\mathfrak{C})$ is a 2 , 3-group of order divisible by 3 
such that

(a) $O_{3}(\bar{\complement})=1$.

(b) $\bar{夭}$ contains a four-group.

(c) $\bar{夭}$ contains no noncyclic abelian subgroup of order 8 .

It is routine to verify that $\overline{\mathfrak{F}} \cong G L(2,3)$ or $\overline{\mathbb{S}} \cong \Sigma_{4}$ or $\overline{\mathfrak{S}} \cong A_{4}$. If $G L(2,3) \cong \overline{\mathbb{C}}$, then every four-subgroup of $\overline{\mathfrak{C}}$ normalizes a $S_{3}$-subgroup of $\overline{\mathbb{C}}$, against the maximality of $\overline{\mathfrak{B}}$. Hence,

$$
\text { (5/O } / \boldsymbol{O}_{3}(\mathfrak{5}) \cong \Sigma_{4} \text { or } A_{4} \text {. }
$$

Let $\Re_{2}^{*}=\langle I, J\rangle$.

Case 1. $J$ does not invert $\Re_{3}$. Let $\mathfrak{X}=C(J) \cap \Re_{3}$, so that $\mathfrak{X}=$ $\boldsymbol{C}\left(\Re_{2}^{*}\right) \cap \Re_{3}=\boldsymbol{O}_{3}(\mathfrak{E}) \cap C\left(\Re_{2}^{*}\right)$ is of order 3. By a formula of Wielandt [40], together with Lemma 9.30, we get $\left|\boldsymbol{O}_{3}(\mathfrak{S})\right| \leqq 3^{4}$. Since $\boldsymbol{O}_{3}(\mathbb{E})=$ $\boldsymbol{F}(\mathfrak{C})$, it follows from $(B)$ that $m\left(\boldsymbol{O}_{3}(\mathbb{E})\right) \geqq 3$. Hence, $\boldsymbol{O}_{3}(\mathbb{E})$ is elementary of order $3^{3}$ or $3^{4}$. If $\left|\boldsymbol{O}_{3}(\mathfrak{E})\right|=3^{3}$, then $\boldsymbol{O}_{3}(\mathfrak{E})$ char $\mathfrak{F}_{3}$, and so $\mathbb{F}_{3}$ is a $S_{3}$-subgroup of $\mathbb{B}$, against Lemma 9.31. Hence, $O_{3}(\mathbb{E})$ is elementary of order $3^{4}$. This implies that $\boldsymbol{O}_{3}(\mathfrak{S})$ char $\mathfrak{F}_{3}$. Hence, $\mathbb{F}_{3}$ is a $S_{3}$-subgroup of Ss. This is not the case, since $Z\left(\mathbb{F}_{3}\right)$ is noncyclic.

Case 2. $J$ inverts $\Re_{3}$ and $\overline{\widetilde{C}} \cong \Sigma_{4}$.

Let $\mathfrak{B}=\mathfrak{F}_{2} \cap \boldsymbol{O}_{3,2}(\mathfrak{E})$. Thus, $\mathfrak{B}$ is a four-group. Suppose $\mathfrak{B}=\mathfrak{R}_{2}^{*}$. Let $\mathfrak{S}=\mathfrak{M} \cap \mathfrak{S}$. Then $|\mathfrak{S}|=8.9$, and $\Re_{3} \triangle \mathfrak{S}$. This is not the case, since $\Re_{2}^{*}$ is a four-group, by Lemma 9.27 .

Since $\mathfrak{B} \neq \Re_{2}^{*}$, it follows that $\mathfrak{B}$ and $\Re_{2}^{*}$ are the four-subgroups of $\mathfrak{夭}_{2} \quad$ By Lemma $9.32, \boldsymbol{A}_{\mathscr{G}}\left(\Re_{2}^{*}\right)=\operatorname{Aut}\left(\Re_{2}^{*}\right)$. Thus, $\mathfrak{B} \cap \Re_{2}^{*}=\langle V\rangle$ with $V \sim I$. Hence, all involutions of $\mathfrak{B}$ are conjugate to $I$ in $\mathbb{B}$.

Choose $V$ in $\mathfrak{B}^{\sharp}$. Suppose $\left|\boldsymbol{C}(V) \cap \boldsymbol{O}_{3}(\mathfrak{C})\right|>3$. Then $\boldsymbol{C}(V) \cap \boldsymbol{O}_{3}(\mathfrak{C})$ is a $S_{3}$-subgroup of $C(V)$, by Lemma 9.29, together with $V \sim I$. Hence, $\left|C_{\mathfrak{g}}(V)\right|=8.9$, and $C_{\mathfrak{g}}(V)$ is 3-closed. This violates Lemma 9.27 applied to $C(V)$. Hence, $\left|\boldsymbol{O}_{3}(\mathfrak{S}) \cap(V)\right| \leqq 3$.

Since $N(\mathfrak{B}) \cap \mathbb{E}$ permutes transitively the involutions of $\mathfrak{B}$, we get $\left|C(V) \cap \boldsymbol{O}_{3}(\mathfrak{S})\right|=3$ for all $V$ in $\mathfrak{B}^{\sharp}$. Hence, $\left|\boldsymbol{O}_{3}(\mathfrak{B})\right|=27$ and $\boldsymbol{O}_{3}(\mathfrak{S})=$ $J\left(\mathfrak{\Im}_{3}\right)$ char $\mathfrak{\mho}_{3}$. But then $|\mathfrak{夭}|_{3}=|\mathbb{S}|_{3}$, against Lemma 9.31.

Case 3. $J$ inverts $\Re_{3}$ and $\overline{\mathbb{S}} \cong A_{4}$.

Let $\mathfrak{E}_{0}=\boldsymbol{O}_{3}(\mathfrak{E})$. Then $\Re_{3}=\boldsymbol{C}_{\mathfrak{E}_{0}}(I)$ is elementary of order $3^{2}$ and inverts by each element of $\Re_{2}^{*}-\langle I\rangle$. Since the involutions of $\Re_{2}^{*}$ are fused in 5 , we conclude

(a) for each $K \in\left(\Re_{2}^{*}\right)^{\ddagger}$, the group $C_{\mathbb{\mathfrak { S }}_{0}}(K)$ is elementary of order $3^{2}$ and is inverted by each element of $\mathscr{R}_{2}^{*}-\langle K\rangle$.

It follows that

(b) $\mathfrak{S}_{0}$ contains two chief factors of $\mathfrak{E}$, each of order $3^{3}$. 
Suppose that $\mathfrak{F}_{0}$ is abelian. By (a) and (b), it is elementary of order $3^{6}$ and each element of $\mathbb{E}_{3}-\mathbb{E}_{0}$ has minimal polynomial $(x-1)^{3}$ on $\mathfrak{E}_{0}$. Hence, $\mathfrak{E}_{0}$ char $\mathfrak{E}_{3}$. So $\mathbb{E}_{3}$ is a $S_{3}$-subgroup of $\mathbb{E}$. But $Z\left(\mathbb{E}_{3}\right)=$ $\left[\mathfrak{C}_{0}, \mathfrak{C}_{3}, \mathfrak{C}_{3}\right]$ is not cyclic, against hypothesis (iii) of Theorem 9.1. Therefore, $\mathbb{C}_{0}$ is not abelian. So (a) and (b) imply:

(c1) $\mathfrak{C}_{0}$ is special of order $3^{6}$ and exponent 3,

(c2) $\boldsymbol{D}\left(\mathfrak{S}_{0}\right)=\boldsymbol{Z}\left(\mathfrak{S}_{0}\right)$ is a chief factor of $\mathfrak{E}$ of order $3^{3}$,

(c3) $\mathbb{E}_{0} / \boldsymbol{D}\left(\mathfrak{E}_{0}\right)$ is a chief factor of $\sqrt{5}$ of order $3^{3}$,

(c4) every element of $\mathfrak{E}_{3}-\mathfrak{S}_{0}$ has minimal polynomial $(x-1)^{3}$ on both $\boldsymbol{D}\left(\mathfrak{F}_{0}\right)$ and $\mathfrak{F}_{0} / \boldsymbol{D}\left(\mathfrak{F}_{0}\right)$,

(c5) if $P \in \mathbb{E}_{3}-\mathfrak{E}_{0}$, then $\left|\boldsymbol{C}_{\mathfrak{E}_{3}}(P)\right| \leqq 3^{3}$.

This implies

(d) $\mathfrak{V}_{0}$ char $\mathfrak{E}_{3}$.

Indeed, if $\mathfrak{F}_{1}$ is any subgroup of index 3 in $\mathfrak{F}_{3}$ different from $\mathfrak{S}_{0}$, then $\mathfrak{E}_{1} \cap \mathfrak{S}_{0} \supseteqq \boldsymbol{D}\left(\mathfrak{S}_{0}\right)$. Hence, (c4) implies that the exponent of $\mathfrak{S}_{1}$ is 9 . This proves $(\mathrm{d})$, and gives

(e) $\mathbb{C}_{3}$ is a $S_{3}$-subgroup of (B).

Now let $\mathfrak{A}_{0}$ be a subgroup of $\Re_{3}$ of order 3 such that $\boldsymbol{C}_{\mathfrak{\Re}_{0}}\left(\mathfrak{A}_{0}\right)=$ $\mathfrak{Q} \supset\langle I\rangle$. Let $\mathfrak{夭}_{3}=A_{0} \times A_{1}$. Thus, $\mathfrak{Q}$ is a quaternion group and $\mathfrak{Q} \mathfrak{A}_{1}\langle J\rangle \cong G L(2,3)$. Let $\mathbb{2}$ be a $S_{2,3}$-subgroup of $C_{\oplus}^{*}\left(\mathfrak{A}_{0}\right)$ with $\mathfrak{R}_{3}\langle J\rangle \cong L$. Let $\mathbb{R}_{0}=\boldsymbol{O}_{3}(\Omega)$. Since $\boldsymbol{D}\left(\mathfrak{S}_{0}\right) \Re_{3}$ is elementary of order $3^{4}$ and contains an element of $\mathscr{C}(3)$, it follows that $O_{3^{\prime}}(\mathbb{R})=1$. Since $\mathbb{Q}$ contains no noncyclic abelian subgroup of order 8 , we get that $\mathfrak{Q} \mathfrak{U}_{1}\langle J\rangle$ is a complement to $\mathfrak{L}_{0}$ in $\mathfrak{L}$. Since $I$ inverts $\mathfrak{L}_{0} / \mathfrak{A}_{0}$, it follows that $\left|\mathfrak{R}_{0}: \mathfrak{A}_{0}\right|=3^{2 d}$ for some integer $d \geqq 1$. If $d=1$, then $\boldsymbol{D}\left(\mathfrak{S}_{0}\right) \Omega_{3}$ is a $S_{3}$-subgroup of $C\left(\mathfrak{N}_{0}\right)$ and so $S_{3}$-subgroups of $\mathbb{R}$ are abelian. This is absurd, so $d \geqq 2$. Since $\left.|| S\right|_{3}=3^{7}$ by (e), and since $|\&|_{3}=3^{2 d+2}$, we get $d=2$.

Let $\Omega_{3}$ be a $S_{3}$-subgroup of $\mathbb{Q}$ containing $\Omega_{3}$. Since $\Omega_{3}$ is not a $S_{3}$-subgroup of $\mathbb{S}$, and since $\mathfrak{A}_{0} \triangle \mathscr{Q}$, it follows that $\boldsymbol{Z}\left(\mathbb{R}_{3}\right)$ is noncyclic. In particular, $\boldsymbol{Z}\left(\Omega_{0}\right) \supseteqq \boldsymbol{Z}\left(\mathbb{\Omega}_{3}\right)$, so that $\boldsymbol{Z}\left(\Omega_{0}\right)$ is not cyclic. Hence, $\left|\boldsymbol{Z}\left(\Omega_{0}\right)\right| \geqq 3^{3}$. This implies that if $L \in \mathbb{\Omega}_{0}$, then $\left|\boldsymbol{C}_{\mathfrak{Q}_{0}}(L)\right| \geqq 3^{4}$. Choose $G$ in (S) so that $\Omega_{3} \subseteq \mathfrak{C}_{3}^{G}$, which is possible by (e). By (c5), we get $\mathfrak{\Omega}_{0} \subseteq \mathfrak{C}_{0}^{G}$. Hence, $\mathbb{\Omega}_{0}=\mathfrak{\Omega}_{00} \times \mathfrak{\Omega}_{01}$, where $\mathfrak{\Omega}_{00}, \mathfrak{\Omega}_{01}$ admit $\mathfrak{\Omega}, \mathfrak{\Omega}_{00}$ is nonabelian of exponent 3 and order $3^{3}$ and $\Omega_{01}$ is elementary of order $3^{2}$. Now $\mathfrak{A}_{1} \subseteq \mathfrak{R}_{3}$ and $\mid \boldsymbol{C}_{\mathfrak{Q}_{3}}\left(\mathfrak{A}_{1}\right) \geqq 3^{4}$, so we get that $\mathfrak{U}_{1} \subseteq \mathfrak{C}_{0}^{G}$. Hence, $\mathfrak{尺}_{3}=\mathfrak{Q}_{0} \mathfrak{U}_{1} \subseteq \mathfrak{\mho}_{0}^{G}$, and so $\mathfrak{Q}_{3}=\mathbb{\mathfrak { S }}_{0}^{\sigma}$. This is impossible, since $\left|\boldsymbol{Z}\left(\mathfrak{Q}_{3}\right)\right|=3^{2},\left|\boldsymbol{Z}\left(\mathfrak{S}_{0}\right)\right|=3^{3}$.

LEMmA 9.34. Each involution of $\Re_{2}^{*}-\langle I\rangle$ inverts $\Re_{3}$.

Proof. Let $\mathfrak{B}^{*}$ be a $S_{2}$-subgroup of $(S)$ which contains $\Re_{3}$ and is normalized by $\Re_{2}^{*}$, set $\mathfrak{X}=\Re_{3} \cap \boldsymbol{C}\left(\Re_{2}^{*}\right)$. Suppose $\mathfrak{X} \neq 1$. Then $|\mathfrak{X}|=3$, so by a formula of Wielandt [44], $\left|\mathfrak{P}^{*}\right| \leqq 3^{4}$. This contradicts Lemma 9.31. Hence, $\mathfrak{X}=1$. As $\Re_{2}^{*}=\langle I, J\rangle$ for some involution $J$, the proof is complete. 
Lemma 9.35. (a) If $\mathfrak{X}$ is a subgroup of $\Re_{3}$ of order 3 and $\boldsymbol{C}(\mathfrak{X}) \cap \Re_{0}$ is quaternion, then $|\boldsymbol{C}(\mathfrak{X})|_{3}=3^{4}$.

(b) $|C(\Re)|_{3}=3^{3}$.

Proof. (a) Set $\mathfrak{Q}=\boldsymbol{C}(\mathfrak{X}) \cap \Re_{0}$, and let $\mathfrak{Y}$ be a subgroup of $\Re_{3}$ of order 3 distinct from $\mathfrak{X}$. Let $J$ be an involution of $\Re_{2}^{*}-\langle I\rangle$. Thus, $J$ inverts $\Re_{3}$ by Lemma 9.34. Also, $\langle J\rangle \mathfrak{Y} \cong \cong G L(2,3)$.

Let $\mathbb{S}$ be a $S_{2,3}$-subgroup of $N(\mathfrak{X})$ which contains $\Omega_{3} \mathfrak{S} \Omega_{2}^{*}$. Thus, $\mathfrak{Q}\langle J\rangle$ is a $S_{2}$-subgroup of $\mathbb{E}$ and $O_{2}(\mathfrak{C})=1$. Since

$$
\left[O_{3}(\mathfrak{S}) \cap \Re_{3}, \mathfrak{Q}\right] \subseteq O_{3}(\mathfrak{S}) \cap \mathfrak{l}=1,
$$

it follows that $\boldsymbol{O}_{3}(\mathfrak{E}) \cap \Re_{3}=\mathfrak{X}$. Hence, $I$ inverts $\boldsymbol{O}_{3}(\mathfrak{E}) / \mathfrak{X}$. Hence, $\boldsymbol{O}_{3}(\mathfrak{E}) / \mathfrak{X}$ is the direct sum of a certain number, say $k$, of modules each isomorphic to the faithful irreducible $F_{3} \mathfrak{Q}$-module, so that $\left|\boldsymbol{O}_{3}(\mathbb{E}): \mathfrak{X}\right|=$ $3^{2 k}$. Hence, $|\mathfrak{C}|_{3}=|\boldsymbol{C}(\Re)|_{3}=3^{2(k+1)}$. Suppose $k \geqq 2$. Then by Lemma 9.33, we get $|\mathfrak{C}|_{3}=\mid\left(\left.\mathbb{S}\right|_{3}=3^{6}\right.$.

We argue that $\boldsymbol{Z}\left(\boldsymbol{O}_{3}(\mathfrak{E})\right)=\mathfrak{X}$. Suppose false. We get $\boldsymbol{Z}\left(\boldsymbol{O}_{3}(\mathfrak{E})\right)=$ $\left(\boldsymbol{Z}\left(\boldsymbol{O}_{3}(\mathbb{E})\right) \cap \boldsymbol{C}(I)\right) \times\left[\boldsymbol{Z}\left(\boldsymbol{O}_{3}(\mathfrak{E})\right), I\right]$. Since $\Re_{3} \cap \boldsymbol{O}_{3}(\mathfrak{E})=$, we get

$$
\boldsymbol{Z}\left(\boldsymbol{O}_{3}(\mathfrak{S})\right) \cap \boldsymbol{C}(I)=\mathfrak{X} ;
$$

also $\left[\boldsymbol{Z}\left(\boldsymbol{O}_{3}(\mathfrak{C})\right), I\right]$ is normalized by $\mathfrak{Y}$, so if $\left[\boldsymbol{Z}\left(\boldsymbol{O}_{3}(\mathfrak{S})\right), I\right] \neq 1$, then a $S_{3}$ subgroup of $\mathbb{S}$ has a noncyclic center. We conclude that $\mathfrak{X}=\boldsymbol{Z}\left(\boldsymbol{O}_{3}(\mathfrak{E})\right)$. This implies that $O_{3}(\mathfrak{E})$ is extra special of width 2. Since $\cong\langle J\rangle$ is a $S_{2}$-subgroup of $N(\mathfrak{X})$, it follows that $\boldsymbol{O}_{3}(\mathfrak{C})=\boldsymbol{O}_{3}(N(\mathfrak{X}))$. Thus, Hypothesis 9.2 is satisfied. Since this is not the case, we get $k=1$. Thus (a) holds.

By Lemma 9.20, we have $\left|\boldsymbol{C}\left(\Re_{3}\right)\right|_{3} \geqq 27$. Since $\Re_{3}$ is not central in a $S_{3}$-subgroup of $\mathfrak{C}$, (b) follows.

Lemma 9.36. Let $\mathfrak{P}$ be a $S_{3}$-subgroup of (S). Then

(a) $|\mathfrak{P}|=3^{5}$.

(b) $\mathfrak{P} / \boldsymbol{Z}(\mathfrak{P})$ is of maximal class and order $3^{4}$.

Proof. By Lemma 9.33, there is a conjugate $\mathfrak{B}$ of $\mathfrak{R}_{2}^{*}$ which normalizes $\mathfrak{P}$. By Lemma 9.32, all involutions of $\mathfrak{B}$ are conjugate to I. Let $V_{1}, V_{2}, V_{3}$ be the involutions of $\mathfrak{B}$. By Lemma 9.34, $C_{\mathfrak{P}}(\mathfrak{B})=1$. By Lemma 9.29, $\left|\boldsymbol{C}_{\mathfrak{B}}\left(V_{i}\right)\right| \leqq 9$ for $i=1,2,3$. Then by Wielandt [44], $|\mathfrak{P}| \leqq 3^{6}$.

Set $3=\boldsymbol{Z}(\mathfrak{P})$. Since 3 is cyclic, $\boldsymbol{C}(\mathbb{3}) \cap \mathfrak{B} \neq 1$. We may assume notation is chosen so that $V_{1}$ is a generator for $C(3) \cap \mathfrak{B}$. Thus, $|3|=3$. Suppose $V_{1}$ inverts $\mathfrak{P} / 3$. Then, $|\mathfrak{P}| \leqq 3^{5}$, so by Lemma 9.31 , $|\mathfrak{P}|=3^{5}$. In this case, since $\mathfrak{P}$ is generated by elements of order 3 , we get that $3=\mathfrak{P}^{\prime}=D(\mathfrak{P})$. Since $O_{3^{\prime}}\left(N(3)=1\right.$, so also $\left.O_{3^{\prime}}(3) / 3\right)=1$. 
Hence, $\mathfrak{P} \triangleleft N(3)$. Thus, Hypothesis 9.2 is satisfied. Since this is not the case, we conclude that $V_{1}$ does not invert $\mathfrak{B} / \mathbb{3}$.

Let $\mathfrak{U}$ be a subgroup of $\boldsymbol{C}_{\mathfrak{P}}\left(V_{1}\right)$ of order 3 distinct from 3 . Thus, $C_{\mathfrak{P}}\left(V_{1}\right)=3^{\mathfrak{u}}$.

By Lemma $9.35(\mathrm{~b})$, we get $\left|C_{\mathfrak{P}}(\mathfrak{3} \mathfrak{u})\right| \leqq 27$. Since $N_{\mathfrak{\beta}}(\mathfrak{3} \mathfrak{u})=C_{\mathfrak{P}}(\mathfrak{Z} \mathfrak{u})$, we have $\left|C_{\mathfrak{B}}(\mathfrak{Z} \mathfrak{U})\right|=27$. Again, since $N_{\mathfrak{P}}(\mathfrak{Z} \mathfrak{U})=C_{\mathfrak{B}}(\mathfrak{Z U})$, if follows that $\left|N_{\mathfrak{P} / 3}(\mathfrak{Z M} / 3)\right|=9$. Thus, $\mathfrak{P} / 3$ is of maximal class, and $\mathfrak{U} 3 / 3 \nsubseteq(\mathfrak{B} / 3)^{\prime}$. Since $\mathfrak{u} 3 / 3$ is the set of fixed points of $V_{1}$ on $\mathfrak{B} / 3$, it follows that $\mathfrak{B} / 3$ has a subgroup $\mathfrak{P}_{0} / 3$ of index 3 which is inverted by $\mathfrak{B}_{1}$. Since $\mathfrak{P}_{0} / 3$ is generated by elements of order $3, \mathfrak{B}_{0} / 3$ is elementary. If $\left|\mathfrak{P}_{0} / 3\right| \geqq 3^{4}$, then $\mathfrak{P} / 3$ is not of maximal class. Hence, $\left|\mathfrak{P}_{0} / 3\right| \leqq 27$, so by Lemma 9.31 , we have $\left|\mathfrak{B}_{0}: 3\right|=27$. This establishes both (a) and $(b)$.

We may now complete the proof of Theorem 9.1. Let $\mathfrak{P}, \mathfrak{P}_{0}, 3$, $\mathfrak{U}, \mathfrak{B}, V_{1}$ be as above. Thus, $\left|\mathfrak{F}_{0}\right|=3^{4}, \mathfrak{P}_{0} / \mathbb{3}$ is elementary of order 27 and is inverted by $V_{1}$. Being generated by elements of order $3, \mathfrak{P}_{0}$ is of exponent 3. It follows that $\boldsymbol{Z}\left(\mathfrak{P}_{0}\right)$ is not cyclic. Hence, we can choose a subgroup $\mathfrak{W}$ of $Z\left(\mathfrak{B}_{0}\right)$ of order 9 which is normal in $\mathfrak{P} \mathfrak{B}$. Set $\mathfrak{Y}=\mathfrak{M}$. Thus, $\mathfrak{Y}$ is of order 27 and $\mathfrak{Y}$ admits $V_{1}$. Thus, $3 \mathfrak{U} \triangle \mathfrak{Y}$, so $\mathfrak{Y}$ is abelian, since $N_{\mathfrak{F}}(\mathfrak{Z} \mathfrak{U})=C_{\mathfrak{F}}(\mathfrak{Z} \mathfrak{u})$. This implies that $\mathfrak{W} \subset Z(\mathfrak{Z})$, since $\mathfrak{P}=\mathfrak{B}_{0} \mathfrak{U}$. This contradiction completes the proof of Theorem 9.1.

Theorems 8.1 and 9.1 provide a proof of Theorem ES.

A substantial number of simplifications and corrections have been supplied by E. C. Dade.

Received October 23, 1964, and in revised form August 8, 1969. Research supported by a National Science Foundation Grant, GN-530, to the American Mathematical Society. The author also thanks the Sloan Foundation for its extended support.

UNIVERSITY OF CHICAGO CAMBRIDGE UNIVERSITY 


\section{PACIFIC JOURNAL OF MATHEMATICS}

\section{EDITORS}

H. SAMelson

Stanford University

Stanford, California 94305

\section{Richard PIERCE}

University of Washington

Seattle, Washington 98105
J. DugundjI

Department of Mathematics

University of Southern California

Los Angeles, California 90007

BASIL GoRDON*

University of California

Los Angeles, California 90024

\section{ASSOCIATE EDITORS}
E. F. BECKENBACH
B. H. NeumanN
F. WOLE
K. YosHIDA

\section{SUPPORTING INSTITUTIONS}

UNIVERSITY OF BRITISH COLUMBIA

CALIFORNIA INSTITUTE OF TECHNOLOGY

UNIVERSITY OF CALIFORNIA

MONTANA STATE UNIVERSITY

UNIVERSITY OF NEVADA

NEW MEXICO STATE UNIVERSITY

OREGON STATE UNIVERSITY

UNIVERSITY OF OREGON

OSAKA UNIVERSITY

UNIVERSITY OF SOUTHERN CALIFORNIA

\author{
STANFORD UNIVERSITY \\ UNIVERSITY OF TOKYO \\ UNIVERSITY OF UTAH \\ WASHINGTON STATE UNIVERSITY \\ UNIVERSITY OF WASHINGTON \\ * * * * \\ AMERICAN MATHEMATICAL SOCIETY \\ CHEVRON RESEARCH CORPORATION \\ TRW SYSTEMS \\ NAVAL WEAPONS CENTER
}

The Supporting Institutions listed above contribute to the cost of publication of this Journal, but they are not owners or publishers and have no responsibility for its content or policies.

Mathematical papers intended for publication in the Pacific Journal of Mathematics should be in typed form or offset-reproduced, (not dittoed), double spaced with large margins. Underline Greek letters in red, German in green, and script in blue. The first paragraph or two must be capable of being used separately as a synopsis of the entire paper. The editorial "we" must not be used in the synopsis, and items of the bibliography should not be cited there unless absolutely necessary, in which case they must be identified by author and Journal, rather than by item number. Manuscripts, in duplicate if possible, may be sent to any one of the four editors. Please classify according to the scheme of Math. Rev. 36, 1539-1546. All other communications to the editors should be addressed to the managing editor, Richard Arens, University of California, Los Angeles, California, 90024.

50 reprints are provided free for each article; additional copies may be obtained at cost in multiples of 50 .

The Pacific Journal of Mathematics is published monthly. Effective with Volume 16 the price per volume (3 numbers) is $\$ 8.00$; single issues, $\$ 3.00$. Special price for current issues to individual faculty members of supporting institutions and to individual members of the American Mathematical Society: $\$ 4.00$ per volume; single issues $\$ 1.50$. Back numbers are available.

Subscriptions, orders for back numbers, and changes of address should be sent to Pacific Journal of Mathematics, 103 Highland Boulevard, Berkeley, California, 94708.

PUBLISHED BY PACIFIC JOURNAL OF MATHEMATICS, A NON-PROFIT CORPORATION

Printed at Kokusai Bunken Insatsusha (International Academic Printing Co., Ltd.), 7-17, Fujimi 2-chome, Chiyoda-ku, Tokyo, Japan.

* Acting Managing Editor. 


\section{Pacific Journal of Mathematics}

\section{Vol. 33, No. $2 \quad$ April, 1970}

Raymond Balbes and Alfred Horn, Projective distributive lattices ....... 273

John Findley Berglund, On extending almost periodic functions ......... 281

Günter Krause, Admissible modules and a characterization of reduced left

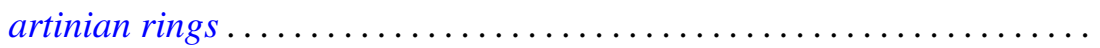

Edward Milton Landesman and Alan Cecil Lazer, Linear eigenvalues and a nonlinear boundary value problem ....................... 311

Anthony To-Ming Lau, Extremely amenable algebras ............... 329

Aldo Joram Lazar, Sections and subsets of simplexes .............. 337

Vincent Mancuso, Mesocompactness and related properties ............. 345

Edwin Leroy Marsden, Jr., The commutator and solvability in a generalized orthomodular lattice .................................. 357

Shozo Matsuura, Bergman kernel functions and the three types of canonical domains.......................................... 363

S. Mukhoti, Theorems on Cesàro summability of series .............. 385

Ngô Van Quê, Classes de Chern et théorème de Gauss-Bonnet ........... 393

Ralph Tyrrell Rockafellar, Generalized Hamiltonian equations for convex problems of Lagrange ................................ 411

Ken iti Sato, On dispersive operators in Banach lattices ............. 429

Charles Andrew Swanson, Comparison theorems for elliptic differential

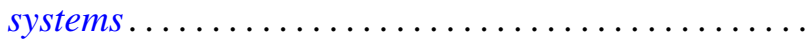

John Griggs Thompson, Nonsolvable finite groups all of whose local subgroups are solvable. II

David J. Winter, Cartan subalgebras of a Lie algebra and its ideals ... 